\title{
A NEW CLASS OF ADDITIVES TO INHIBIT TREE GROWTH IN \\ SOLID EXTRUDED CABLE INSULATION
}

\author{
TD- 145
}

EPRI Project No. RP 7830-1

ERDA Contract No. E (49-18)-1826

Final Report

$$
\text { March } 1976
$$

Prepared by

\author{
Dielectrics Project \\ Power Systems Laboratory \\ General Electric Company \\ Corporate Research and Development \\ Schenectady, New York 12301 \\ Principal Investigators:

$$
\begin{aligned}
& \text { J.C. Devins } \\
& \text { S.J. Rzad } \\
& \text { C.W. Reed } \\
& \text { D.K. Bartosh } \\
& \text { T.W. Stines }
\end{aligned}
$$

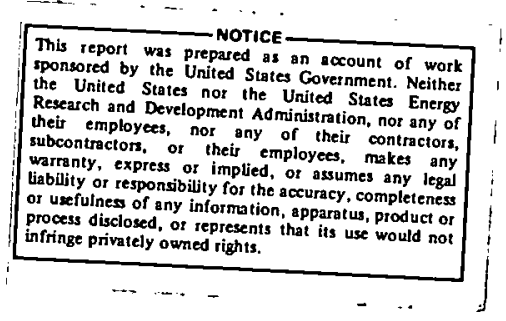

Prepared for

Electric Power Research Institute

3412 Hillview Avenue

Palo Alto, California 94304

and

U. S. Energy Research and Development Administration

Division of Electric Energy Systems

Washington, D.C. 20545 


\section{DISCLAIMER}

This report was prepared as an account of work sponsored by an agency of the United States Government. Neither the United States Government nor any agency Thereof, nor any of their employees, makes any warranty, express or implied, or assumes any legal liability or responsibility for the accuracy, completeness, or usefulness of any information, apparatus, product, or process disclosed, or represents that its use would not infringe privately owned rights. Reference herein to any specific commercial product, process, or service by trade name, trademark, manufacturer, or otherwise does not necessarily constitute or imply its endorsement, recommendation, or favoring by the United States Government or any agency thereof. The views and opinions of authors expressed herein do not necessarily state or reflect those of the United States Government or any agency thereof. 


\section{DISCLAIMER}

Portions of this document may be illegible in electronic image products. Images are produced from the best available original document. 


\section{ABSTRACT}

This report covers the work carried out between May 1, 1974 and August 30, 1975 for the Electric Power Research Institute and the U.S. Energy Research and Development Administration under Project No. RP 7830-1, ERDA Contract No. E(49-18)-1826. It covers the investigation of a new class of additives to inhibit tree growth in solid extruded cable insulation.

The work is reported in two parts:

Part I General Electric Company Corporate Research and Development Center In-House Program:

Development of Techniques for Treeing Evaluation

Part II Program funded jointly by the Electric Power Research Institute and the U.S. Energy Research and Development Administration:

Development of Additives to Inhibit Tree Growth in Solid Extruded Cable Insulation

The in-house program at General Electric Company (Part I) has developed techniques which allow us to follow the growth of trees in polyethylene, by both optical observation through a microscope and measurement of discharge sizes on a.pulse height analyser. We have also developed a needle-molding technique which ensures good reproducibility of the measurements of tree inception voltages. We have examined the influence of needle radius, gap and time on tree inoeption voltages. A correlation between the maximum discharge. size normalized to the applied voltage and tree length has been obtained. Such a correlation should prove very useful in evaluating tree growth in opaque materials.

The principal object of the work funded by EPRI and ERDA (Part II) has been to develop additives, primarily for polyethylene, to, inhibit tree growth utilizing a field grading principle based upon field-enhanced ionic dissociation. During the sixteen months of the contract we have developed a quantitative model which has supplied the framework upon which experi- 
mental work has proceeded. This has led to an examination of a number of types of ionic additives with a view to obtaining maximum solubility and maximum conductivity. The results of this search are presented in this report. Although, in terms of our model, we have obtained sufficient conductivity to have effected marked increases in tree inception voltage, the improvements have been marginal. Possible reasons for this discrepancy are presented and discussed. A second objective of this work has been to conduct experiments designed to verify the fundamental tenets upon which the model rests. To this end we have examined the field dependence of conductivity in liquids and in polymers, with DC and with $A C$ at a number of frequencies. The results in liquids have supported our model in considerable detail, and field grading appears to be well established. In polymers the question is still not resolved. Experiments are proposed which could shed further light on the matter. The use of a chlorinated biphenyl liquid as a second additive to increase the dielectric constant and therefore the degree of dissociation has been investigated. Two additives have been found which markedly inhibit tree formation, although the mechanisms by which this occurs are not clear.. Ferrocene completely prevents treeing under all conditions tested, and increases the breakdown voltage in our test by $100 \%$. N,N'diphenyl p-phenylene diamine increases the treeing time in our long: time test by a factor of about 60 .

Before proceeding to an analysis for use in cables of the more promising additives identified in this work, as proposed in the original contract, it is recommended: that additional data are needed on field grading additives, on the mechanism by which ferrocene operates and on the performance of ferrocene and other most-promising candidates in cross-linked polyethylene. 


\section{PART I}

General Electric In-House Program:

Development of Techniques for Treeing Evaluation

I. INTRODUCTION 2

II. TREEING AND CABLE LIFE 4

III. FIELD GRADING WITH ADDITIVES 12

IV. TECHNIQUES FOR TREEING EVALUATION 16

V. REFERENCES $\quad 35$

$\begin{array}{lll}\text { VI. APPENDICES } & 37\end{array}$

Appendix 1 -- The Second wien Effect 37

Appendix 2 - $\begin{gathered}\text { Conductivity and Additive } \\ \text { Concentration }\end{gathered}$

Appendix 3 -- $\begin{aligned} & \text { Heating in the Grading } \\ & \text { Region }\end{aligned}$

Appendix 4 -- Work Statement from Original General Electric Company Corporate Research and Development Technical Proposal (CRD:3351.008E submitted to Electric Power Research Institute in June 1973 on a "New Class of Additives to Inhibit Tree Growth in Solid Extruded Cable Insulation."

\section{PART II}

EPRI-ERDA Funded Program:

Development of Additives to Inhibit Tree Growth

in Solid Extruded Cable Insulation

I. INTRODUCTION 
CONTENTS (Cont'd)

$\underline{\text { Page }}$

PART II (Cont'd)

II. FIELD CALCULATIONS 53

III. CURRENT MEASUREMENTS IN POINT-PLANE GEOMETRIES 60

IV. ADDITIVES 76

V. FREQUENCY DEPENDENCE OF FIELD GRADING 104

VI. REFERENCES 119

VII. APPENDICES

$\begin{array}{lll}\text { Appendix } 1-- & \text { Field Calculation for } \\ & \begin{array}{l}\text { Concentric Sphere } \\ \text { Geometry }\end{array} & 120 \\ \text { Appendix } 2-- & \text { Dielectric Measurements } & 126\end{array}$ 
This final report describes work performed between May 1 , 1974 and August 30, 1975 for the Electric Power Research Institute and the U.S. Energy Research and Development Administration under Project No. RP7830-I, ERDA Contract No. E(49-18)-1826. It includes the four-month extension work carried out between May 1 , 1975 and August 30, 1975. The work was performed and the report was prepared by the Power systems Laboratory at the Corporate Research and Development Center of the General Electric Company, Schenectady, New York. The program is under the technical cognizance of an ERDA-EPRI project steering Committee, whose chairman is Mr. John W. Gannon, Boston Edison Company. Useful discussions with, and inputs and suggestions by, the steering committee are gratefully acknowledged. Members of that Committee were:

J. W. Gannon (Boston Edison Company), Chairman

J. T. Corbett (Detroit Edison Cumpany)

H. Feibus (ERDA)

F. G. Garcia (EPRI)

S. V. Heyer (Philadelphia Electric Company)

P. L. Kolarik (EPRI)

R. P. Mott (Cleveland Electric Illuminating Company)

S. P. Walldorf (ERDA)

The work described in this report was carried out partly under funding by EPRI and ERDA, and partly under an in-house program at the General Electric Company Corporate Research and Development center. The in-house program carried out at the General Electric Company Corporate Research and Development Center is covered in Part $I$. The work carried out under funding from EPRI and ERDA is covered in Part II.

The principal contributors to this work were:
J. C. Devins
S. J. Rzad
C. W. Reed
D. K. Bartosh
T. W. Stines 
$\underline{\text { PART I }}$

General Electric Company Corporate Research and Development In-House Program:

DEVELOPMENT OF TECHNIQUES FOR TREEING EVALUATION 


\section{INTRODUCTION}

The ability to completely inhibit tree growth in solid extruded cable dielectrics subject to a wide range of operating and environmental conditions is probably the key to successfully obtaining service life of solid extruded cables comparable to the 30-40 years obtainable with oil-impregnated paper cables. This is particularly true at voltages of 69 and $138 \mathrm{kV}$ and higher. While improved success in this endeavor can be expected through extreme cleanliness, improved processing, and the use of co-extruded shields, the intrinsic structure of the resulting solid dielectric will remain highly susceptible to electrical deterioration when subjected to high localized electric fields and internal discharges. It is our belief that highly reliable methods need to be developed to eliminate the effect of those factors that cause trees to initiate and propagate. A theoretical treatment described in section III led us to believe that this could be achieved through stress grading using a new class of additive materials.

Our original proposal, submitted in June, 1973 by the Dielectrics Project of General Electric Company's Corporate Research and Development Center to the Electric Power Research Institute and the Energy Research and Development Administration, offered a program (1) to demonstrate the effectiveness and mechanism by which this new class of additives inhibits tree growth in solid extruded dielectrics, and (2) to develop these additive systems for cable application. The work covered in this report was directed to item 1, a so-called Phase 1 program to demonstrate the effectiveness and mechanism by which the new class of additives inhibited tree growth in solid extruded cable dielectrics. It was anticipated that the work of developing such additives for cable application would be performed in a Phase 2 program, which would be submitted as a separate proposal. The (Phase 1) work was comprised of two parts: 
Part 1 General Electric Company Research and Development Center In-House Program:

Development of Techniques for Treeing Evaluation

Part 2 Program funded jointly by the Electric Power

Research Institute and the U.S. Energy Research and Development Administration:

Development of Additives to Inhibit Tree Growth in Solid Extruded Cable Insulation

The present part (Part 1) will describe the basis for looking at stress grading additives (Section III: Field Grading with Additives) and then describe the various techniques investigated for evaluating treeing (Section IV). 


\section{TREEING AND CABLE LIFE}

There is now substantial evidence that in many dielectric failures of solid polyolefinic and other polymeric materials the final disruption may be preceded by the long-time progressive development of a three-dimensional pattern of irregular, sometimes (though not always) carbonized hollow channels diverging from a central stem, and that the ultimate failure follows one of these channels. These minute channels are referred to as "trees" (or sometimes, but less frequently, "dendrites") and the phenomenon is described generically as "treeing."

The increased use of these solid polyolefinic and other polymeric materials for extruded underground cables (5-138 kV) for distribution and industrial purposes has led, in the last four years, to a realization of the importance of treeing as a mode of cable degradation that can lead to premature failure of the cable. In studies carried out by Pacific Gas and Electric Company on the west coast of the USA (1) on 161 (15 and $22 \mathrm{kV}$ ) polyethyleneinsulated cables contributed by 28 utility companies, sections of failed and non-failed cables that had been in service from less than one year to eleven years were examined and revealed widespread incidence of treeing, particularly in cables energized more than 5 years. The study concluded that many polyethylene cables in service are likely to contain trees; however, it refrained from a correlation between treeing and cable life. Further, the study showed that, while certain improvements in extrusion procedures, cleanliness, and the use of extruded shields led to improvements in the resistance to treeing, under sufficiently adverse environmental conditions trees still eventually initiate, propagate, and lead to cables with significantly deteriorated electrical performance.

The important practical conclusion from these and other related investigations is that treeing is directly associated with reduced voltage-life, and that the situation will likely become significantly worse at higher voltages $(69,138 \mathrm{kV}$, and 
above) when it will be desirable to go to higher stress levels at the inner conductor. Thus, to improve voltage-life to 30 years or more, we consider that it is essential to completely eliminate treeing as a mode of cable degradation, and the ultimate objective of this proposed study was to eliminate or significantly inhibit treeing through the development of a new class of additives. In the next section we will describe the theoretical basis for our belief that these additive systems will perform to suppress tree initiation and growth. In the following sections we describe an experimental program to demonstrate the effectiveness and mechanism of these additives--which we believe are quite unlike those examined by other investigators--and to develop their use for cable application.

Some of the earlier investigations on treeing $(2-5)$ in the 1950's revealed several of the more significant features of one major class of trees: the so-called "electrical trees." In recent years, marked by some thorough and excellent work in Japan, two new classes of trees have been identified: "water trees" (6-8) and "chemical trees."(9) Indicative of the importance ascribed in Japan to the role of trees in the performance of solid extruded cable insulation, was the establishment of a Japanese IEE Study Committee on Treeing, composed of Japanese industry, government and universities. For those familiar with the Japanese language, reports of the Japanese IEE Study Committee are available. (10)

Several recent summaries of the situation with respect to treeing have been presented at Symposia at the Conference on Electrical Insulation and Dielectric Phenomena. (11-13) The Symposia covered both fundamental and practical aspects of treeing in rapporteur-style presentations, and the individual papers and the rapporteurs' analyses of the papers form a comprehensive introduction to the field. The fundamental papers examined various aspects of the mechanism of treeing, often using needles inserted into the insulation or with deliberately introduced voids. The practical aspects covered water treeing (sometimes 
called penetration treeing), chemical treeing, the use of additives and fillers to suppress treeing, and an evaluation of treeing in cables removed from service. This latter group of papers gives a very useful assessment of the severity of the practical problem of treeing in underground solid extruded cables.

\subsection{Mechanisms of Treeing}

At this time, a clear understanding of the mechanism of treeing remains to be obtained. However, there are some general characteristics of all the suggested mechanisms of treeing--even though some of them are highly speculative--so that we consider that the additive systems we are considering offer the basis for an effective control against all types of trees. We will review in qualitative terms the basic features of the several suggested mechanisms of treeing. With electrical trees, the trees initiate from high stress concentrations at asperities (on the shields) discontinuities, or inclusions, or from voids. In the absence of voids, the action of the localized high electric field (at an asperity or inclusion) can lead to treeing in two ways. If the stress exceeds the intrinsic strength of the material, localized breakdown occurs and a void elongated in the field direction is formed. Discharges in this void transfers the high field to the void tip and further breakdown can occur to extend the void into a thin hollow tubule. Successive discharges and breakdown lead to extension of the tubule, or treeing. If the stress does not exceed the intrinsic strength of the material, there is an induction period before the periodic mechanical stress due to the ac voltage leads to the production of an elongated void. Thereafter the tree develops from the action of discharge columns in the tubule, as in the case of a tree initiated by intrinsic breakdown. For trees arising from high localized fields, some authors suggest that erosion of the dielectric caused by the discharges in the tubules plays a dominant role in the propagation, but this view is not supported by the almost complete lack of carbonization in many instances. The carbonization that is found with such trees is generally thought to occur only in the later 
stages of growth. However, where there are voids (of sufficiently large size) originally present in the insulation, erosion plays a dominant role in the tree initiation. Unlike the cases above, discharges are detectable immediately and they quickly lead to erosion of the void surfaces. Eventually the erosion concentrates at localities where pits form. Then as the pits grow, the discharges increase in energy, and the pit develops into narrow tortuous channels or tubules. This erosion, pitting, and tubuleformation sequence is characteristic of trees originating from voids. However, the propagation of trees originating from voids can also take place via an intrinsic breakdown or electromechanical action. Water trees, and chemical trees are formed at lower voltage stresses and are associated with the penetration of water and water-soluble impurities into the dielectric. With water trees, formation occurs after some period of time, when the water begins to wet the polymer and accumulate in the fine fissures in the insulation structure. Generally these fissures occur in the vicinity of the shields, and under the action of an ac field the water tree penetrates further into the insulation. Penetration of the water tree is accompanied by a progressive drop in insulation resistance and dielectric strength. However, by heating the insulation, the water can be removed from the water tree and the insulation resistance and dielectric strength can be restored to their original high values. $(6,7)$ Reimmersion of the polymer in water causes an immediate drop in these properties, from which it is inferred that some permanent change, including an enhanced tendency to wet, has occurred within the fissures or tubules of the tree. Initiation and propagation, however, occur as a result of the high localized electric stress at the tip of the water fissure. With chemical trees, chemical reactions between salts dissolved in the water and the electrode metal can lead to dendrite or tree formation at exceptionally low fields or even in the absence of voltage. It is possible to eliminate such chemical trees by chemical protective means. 
Thus, we find that both electrical and water trees have one significant common feature: In order to initiate or propagate, they both require the development of a very high localized electric stress. The new class of additives considered in this report is designed to automatically suppress the development of such high localized electric fields." This contrasts with the function of the additive systems used by previous investigators. To emphasize this difference, a brief review of the development and function of previous additive systems follows.

\subsection{Previous Investigations on Additives}

Voltage stabilizing additives are just one of a number of different ingredients that are used in different solid extruded polymeric insulations depending upon the type of cable, the duty cycle it will see, and the balance of properties that are needed. compounding agents such as oils and waxes, antioxidants and catalyst stabilizers, and mineral fillers of low hygroscopicity may all be used. A large variety of additives, including various antioxidants, have been tried specifically for the purpose of improving voltage life: hence the origin of the terms "voltage stabilizers" or "voltage stabilizing additives." The description "anti-track additives" has also been used for additives to improve the voltage performance of cables, but the description is borrowed from other applications of the same material. The simple description "additive" is now widely used to mean voltagestabilizing additives and it will be used in this report. Often the specific additives may be proprietary and its chemical constitution and proposed function are not disclosed.

Most of the theoretical justification of the mechanism by which previous additives are considered to work relate to the suppression of ionization in voids. Broadly speaking, there are. two schools of thought on how this occurs. The first suggests that the additive coats the wall of the void with a film that is either inherently sufficiently conducting or, as a result of some internal discharges, becomes sufficiently conducting, so 
that the void is "shorted out." The second claims that the additive diffuses into the void and reduces its volume, in some cases--as with unsaturated hydrocarbons--acting as a stabilizing, hydrogen absorbing liquid, to protect the polymer from bombard-. ment. However, with most investigations, the evidence for making. a distinction between the two effects is fragmentary.

By far the most thorough investigation of the first category; was by investigators at the Furukawa Electric Company in. Japan (14) who have calculated the surface resistivities required to. "short out" spherical voids of varying size, and then proceeded to identify several series of semi-conducting materials (amines, carbamates, disulphides, etc.) which eliminated internal dis-. charges and inhibited the tendency to treeing: in sheets of crosslinked polyethylene with artificial voids. The same additives showed similar benefits in short- and long-time tests on cable; models.

It was originally thought that the same mechanism was responsible for the significantly improved resistance to treeing obtained when NN' diphenyl paraphenylene diamine (one of the constituents in the "staining" antioxidant Akroflex) was added to polyethylene and tested in the single-needle/remote-ground system of Kitchen and Pratt. $(4,15)$ It was later revealed that the likely explanation for their results ${ }^{(16)}$ lay in the surface "blooming" of the antioxidant, which coated the entire sample.. surface and in the presence of moisture acted as a Faraday cage. thus reducing the stress at the needle point. However, it should be noted that such an action would not be effective. in a cable. construction.

To circumvent this experimental problem, McMahon and Perkins ${ }^{(17)}$ used a double needle/interior-ground system which molded into the sample two needle electrodes that face one another--one at high potential, the other at ground potential.: An improved resistance to treeing was observed with unspecified additives present in polyethylene, and the authors interpret the action of the additives as a coating of void surfaces which 
inhibits the initiation of treeing by gaseous discharges in such voids. Unfortunately, McMahon and Perkins' data on the growth rate of trees with and without these additives, suggest that the additives cause an induction period before treeing begins but thereafter growth proceeds quite rapidly.

Similar experiments have been done by Mason $(11,18)$ on additives of non-staining antioxidants, plasticers and halogenated aromatic carbocyclic materials. Mason found poor reproducibility of his data using a needle point, and he developed specimens with conical recesses coated with evaporated aluminum, to obtain improved results. To make comparisons, he used discharge initiation voltage and time to breakdown. Relative to the base polymer, the antioxidant was inferior in both respects, the plasticizer was inferior in discharge inception but superior in the time to breakdown, and the halogenated additive was superior in discharge inception but inferior in the time to breakdown.

Hayami and Yamada (11) have interpreted the results of their experiments on the effect of additives on treeing in PE and XLPE using needle electrodes in terms of the filling of voids at the needle tip. Acetophenone, cable oil, polybutene, tri-octyltrimellitate, and 40-chlorinated-paraffin all raised the voltage at which trees appeared. The improvements obtained correlated quite well with the solubility of these liquids in the PE or XLPE. With silicone oil, only a very low viscosity (10 centistokes) showed any solubility or any corresponding improvement in treeing resistance. A single needle technique was used, but the base of the polymer slabs was grounded and an ambient of dry air was used; thus we surmise that the results do not suffer from a Faraday cage effect. Hayami (19) has also described the application of the low viscosity silicone fluid to a $66 \mathrm{kV} 100 \mathrm{~mm}^{2}$ XLPE cable. It is stated that the silicone, which is forced down the hollow or stranded conductor, maintains a void-free interface between the conductor and the insulation, leading to excellent voltage stability. 
A number of other investigators have looked at the use of (often unspecified) additives, but there is no indication that the supposed function of their additives differed from that outlined above, nor did the additives appear to provide any significant improvements in voltage performance.

A point to remember is that the above experiences with additives refer specifically to treeing examined in air: hence, the applicability of the findings to water environments cannot be judged. However, an isolated study by Miyashita et al., (20) with an unspecified voltage stabilizer that was effective against trees in air, showed that the same additive was ineffectual against water trees.

From the foregoing brief description of the work to date on additives, it is suggested that the present understanding of the function of these additives leaves much to be desired. Some fairly-well established techniques for examining tree initiation and growth are now available or are in the process of development and it appears to be an opportune time to develop new and improved classes of additives for the purpose of achieving improved, reliable voltage life for solid extruded cables. 


\section{FIELD GRADING WITH ADDITIVES}

While the details of the mechanism of tree initiation and growth in solid insulation remain somewhat obscure (e.g., the role of discharges or the importance of electromechanical forces are not clear) the necessity for a very high localized field, either at an asperity or inclusion causing tree initiation, or at the end of the growing tree, seems to be in little doubt. The magnitude of this field probably approaches the intrinsic electric strength of the material, i.e., several megavolts/cm. (21) It was the purpose of our proposal to examine methods for modifying the insulation so that field grading would occur, and the buildup of excessive fields in the system would be prevented. We believed that this could be achieved by the addition of small amounts of additives which would not degrade the overall performance of the system. The objective of this work was to explore and demonstrate the applicability of this technique for field grading. Subsequent work would apply and develop the technique, in order to improve the electrical performance of extruded cable insulation. In this section we describe our initial rationale and the reasons for considering that the method was at least feasible.

In order to accomplish this field grading, we proposed to take advantage of the field-dependence of the conductivity of weak electrolytes, the so-called second wien effect. (22) (See Appendix 1). This phenomenon is well-understood and documented (23) and involves the increased dissociation of weak electrolytes into ions with increased electrical fields, producing a higher conductivity in the high field region and therefore a reduction in this field. (We believe that many examples of increased power factor with increased stress in liquid impregnated cables and capacitors may be explained by this phenomenon.) We suggest, therefore the addition of small amounts of weakly dissociating electrolytes to polymers such as those used in extruded cables. Let us examine first the magnitudes of the effects needed and those to be expected. 
Since we are interested primarily in $60 \mathrm{~Hz}$ operation, field grading will occur only if the conductivity in the high field region is sufficiently high so that appreciable ion motion can occur in, say $1 / 4$ cycle. Otherwise, fields will be determined only by dielectric constants. This implies that the relaxation time of the insulation, $\rho \varepsilon$, ( $\rho$ is its resistivity and $\varepsilon$ its dielectric constant) must be about $1 / 240 \mathrm{sec}$. This gives

$$
\rho \leq \frac{10^{13}}{240 \times 2} \approx 2 \times 10^{10} \Omega \mathrm{cm}
$$

We have assumed $\varepsilon=2$. The factor $10^{13}$ puts in the proper units. It should be noted that such a value for $\rho$ gives a loss tangent for the insulation in the high field region of

$$
\tan \delta \approx \frac{10^{13}}{\rho \varepsilon \omega}=\frac{10^{13}}{2 \times 10^{10} \times 2 \times 377}=0.66
$$

an inordinately high value. It is therefore essential that this be accomplished with a loss tangent in the low field region of at least 2 to 3 orders of magnitude less to prevent dielectric heating. We shall examine this question next.

Onsager shows in eqs. 42,39 and 37 of his paper ${ }^{(23)}$ that the ratio of low (zero) field resistivity to high field resistivity is given by*

$$
\rho_{0} / \rho=[F(b)]^{1 / 2}
$$

$$
\begin{aligned}
& \text { where } F(b)=\left(\frac{2}{\pi}\right)^{1 / 2}(8 b)^{-3 / 4} e^{(8 b)^{1 / 2}}\left[1-\frac{3}{8(8 b)^{1 / 2}-}\right. \\
& \left.\frac{15}{128(8 b)}\right] \\
& \text { and } b=9.64 E / \varepsilon T^{2}
\end{aligned}
$$

where $E$ is the field and $T$ is the absolute temperature. If we assume that E must be kept at $2 \times 10^{6} \mathrm{volt} / \mathrm{cm}$ or lower in order

*A physical picture explaining this phenomenon is given in Appendix 1. 
to prevent treeing then

$$
\begin{aligned}
& b=\frac{9.64 \times 2 \times 10^{6}}{2 \times 298^{2}}=108 \text { at } 25^{\circ} \mathrm{C} \text { and } \\
& \mathrm{b}=\frac{9.64 \times 2 \times 10^{6}}{2 \times 358^{2}}=75 \text { at } 85^{\circ} \mathrm{C} .
\end{aligned}
$$

These figures give $[F(b)]_{25}=2.88 \times 10^{10}$ and $[F(b)]_{85}=2.81 \times 10^{8}$ or $\left(\rho_{o}\right)_{25} \leq 3.4 \times 10^{15} \Omega \mathrm{cm}$ and $\left(\rho_{0}\right)_{85} \leq 3.4 \times 10^{14} \Omega \mathrm{cm}$ using $\rho=$ $2 \times 1010 \Omega \mathrm{cm}$. From these low field resistivities the low field loss tangents are

$$
(\tan \delta)_{25} \geq 3 \times 10^{-6} \text { and }(\tan \delta)_{85} \geq 3 \times 10^{-5} \text {. }
$$

From these results we are led to believe that adequately low resistivities may be obtained in the high field region for conductivity grading while maintaining negligible loss tangents in the low field region due to the additives.

We discuss next the nature of the additives and the concentrations required.t Firstly, they must be soluble in the polymer of interest (PE, XLPE, or EPR) which means that they must probably possess fairly large hydrocarbon-like groups. Secondly, the dissolved material must dissociate sufficiently into ions at low field to give the resistivities calculated above, i.e., $10^{14}$ $10^{15} \Omega \mathrm{cm}$. The concentration of ions required to produce these low conductivities is very small, assuming reasonable ionic mobilities. However, the degree of dissociation of the additive is very sensitive to dielectric constant of the medium, being very low for low dielectric constants. For example, the dissociation constant of a typical quaternary ammonium salt is six order of magnitude less in a medium of dielectric constant 2.38 than for one of 3.48 . We shall examine the possibility that additives may

\footnotetext{
${ }^{+}$A more detailed and semiquantitative treatment of this question is given in Appendix 2.
} 
be required to increase the dielectric constant of the insulation; the same result may also be achievable by modifying the polymer.

To summarize:

1. We propose to investigate the addition of weakly dissociating ionic salts to extruded insulating materials for the purpose of grading electric fields and preventing treeing.

2. Such grading depends upon a field grading of the conductivity. Order of magnitude calculations using existing theory strongly support the feasibility of this approach.

3. Additives satisfying the requirements of the theory appear to be commercially available. Additives may also be required to increase the dielectric constant of the insulation slightly as well as add ions to the system.

The above analysis was the basis for the original General Electric Company Corporate Research and Development Center proposal (Technical Proposal : CRD 3351.008E) submitted to the Electric Power Research Institute in June 1973 on "A New Class of Additives to Inhibit Tree Growth in Solid Extruded Cable Insulation." The Work statement from that proposal is shown in Part 1, Appendix 4 of this report. 
IV. TECHNIQUES FOR TREEING EVALUATION

\subsection{Introduction}

The objective of this portion of our work was to develop techniques for measuring the onset voltage of trees and to measure their extent of propagation, primarily to evaluate the effect of additives, but secondarily to provide a tool which might be generally useful in other work. We have combined optical observations of trees growing in situ with simultaneous discharge measurements using pulse-height spectrum analysis. The latter measurements may be made, of course, in opaque samples where direct optical techniques are impractical.

\subsection{Experimental Methods}

Fig. 1 shows a drawing of the cell used for producing and observing trees in polymers. The sample consists of a slab approximately $1 \times .5 \mathrm{x} .1$ inches in size, into which a needle is either molded or inserted parallel to the large plane surface. The gap in which the tree will grow is formed between the point of the needle and the opposite edge of the sample which is coated with silver paint to form the low voltage electrode. Although the gap has been varied, our standard evaluation of additives is conducted using 0.100 inch.

The sample is then placed in a $2.5 \times 2.5 \times 1.5$ inch lucite box, open at the top, fitted on one side by a transistor socket into which the needle is plugged, and connected on the low voltage side to a metal bar held in contact with the silver painted surface by a spring. The box is then filled with a fluorochemical, FC43, supplied by the 3M Co., so that the sample and connections are totally immersed to prevent discharges. The sample, so contained, is supported in the metal box shown in Fig. 1 on the high voltage bus ( 1 " id - $23 / 8$ " od) running into an interlocked high voltage cage. The low voltage connection is made through a feed-through insulator to the pulse height analysis equipment. The sample is illuminated from the bottom 


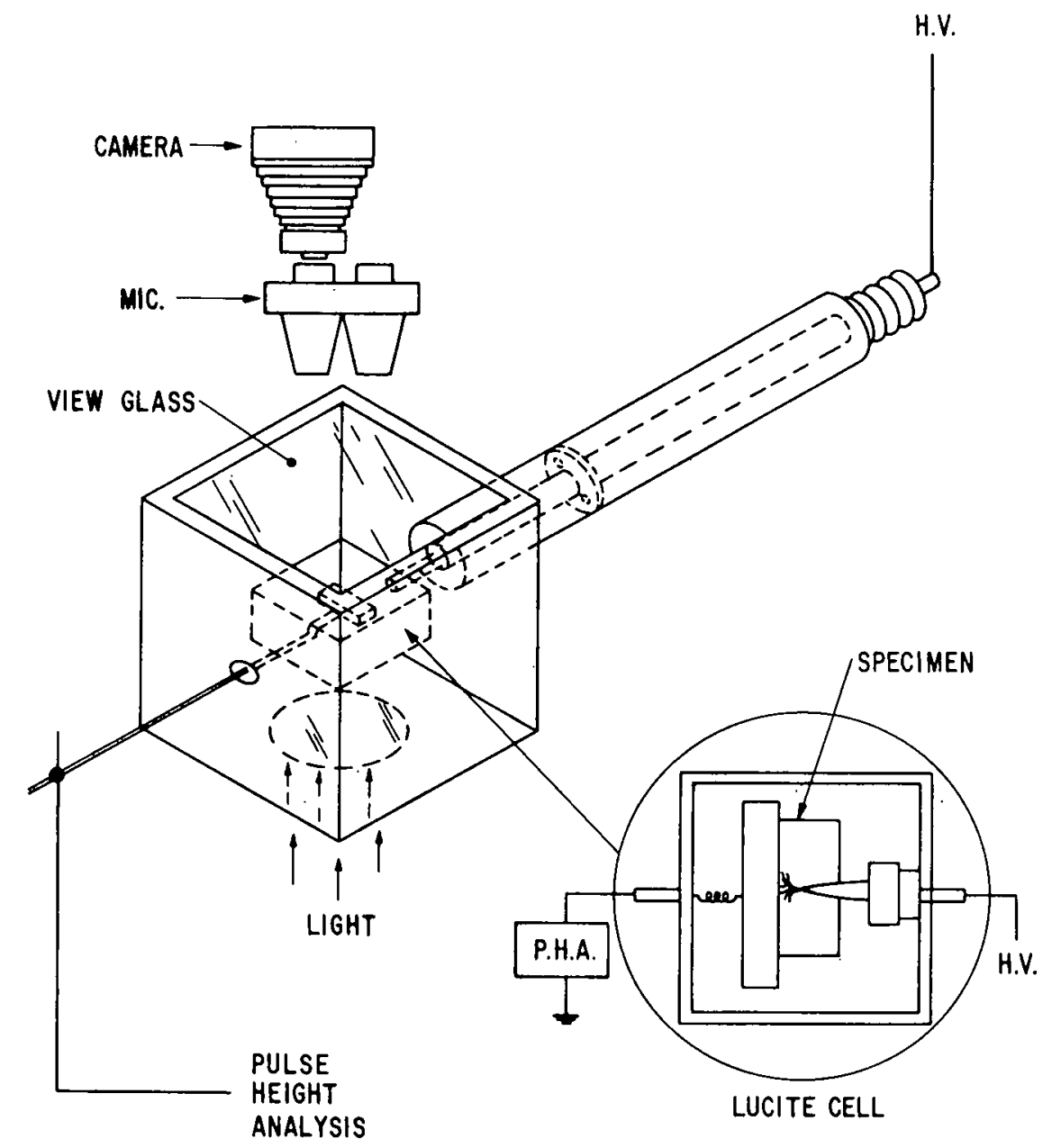

Fig. 1. Experimental apparatus used in tree growth studies. Insert shows the positioning of the sample.

and observed from the top through glass windows coated with transparent, conducting tin oxide kept at ground potential. The top window is loosely gasketed so that the whole can be kept under a slightly positive pressure of $\mathrm{SF}_{6}$ to increase the flashover voltage (between 40 and $50 \mathrm{kV}$ ).

The sample with its growing tree can be observed using a variable magnification ( $\max 16 \mathrm{x}$ ) microscope, and polaroid photographs were taken through the eyepiece. Time lapse motion pictures were also obtained showing the initiation and growth of trees in polymethyl methacrylate'(PMMA), vinyl acetate-ethylene copolymer (PVAP), and polyethylene (PE). Photographs were taken every, five seconds over a period of about forty minutes. Normal projection time was approximately one minute. No difficulty 
was found in observing tree initiation in PVAP and PMMA, but in polyethylene, owing to light scattering from crystallinity considerable "bushing" was required before positive optical identification could be made.

Unless otherwise specified, the needles used in this work were of steel and were individually ground by Horberg Grinding Industries to a $11 / 2 \pm 1 / 4 \mathrm{mil}$ radius. The shadowgraph of a typical needle is traced in Fig. 2. For those experiments in which needle radius was varied, we electrochemically etched standard needles in a $10 \%$ solution of sodium hydroxide using approximately 2 amp $60 \mathrm{~Hz}$ AC current.

Several techniques for inserting needles were investigated. In both PVAP and PE, needles were inserted cold using a jig fitted with a micrometer head so that the gap could be measured. In most cases voids ranging from one to several mils were produced by the relaxation of the needles on removing pressure.

\section{$\underset{5 \text { MILS }}{\longmapsto}$}

Fig. 2. Shadowgraph tracing of a typical needle. Radius $=1.5$ mils.

As will be discussed later, such samples were useful where low tree initiation voltages were desired in order to study tree propagation rates as a function of voltage. A second technique was similar to that aescribed above except that, after insertion, the needles were heated with a soldering gun until melting just appeared at the needle-polymer interface. With such samples, no voids could be detected either visually or due to the presence of 
discharges. The majority of test pieces were prepared by molding in the needles during compression molding of the samples. This procedure appears to produce void-free samples, as judged either optically or electrically.

Discharge magnitudes were measured using the circuit shown in Fig. 3. Current pulses produced by the discharges are shaped by the $1000 \mathrm{pfd}$ and $1000 \mathrm{ohm}$ input circuit to give exponentially decaying signals with a time constant of 1 usec. The back-to-back zener diodes serve to protect the following circuitry when electrical failure of the sample occurs. The signal is then fed through a $50 \mathrm{k} \Omega$ resistor to the open input of a sturrup Linear Amplifier, Model 1410 having a maximum voltage gain of 100 . The output is connected to a Northern Econ II Series Pulse Height Analyzer having 1024 channels with 8 volt maximum channel sensitivity. After a suitable counting period the pulse number-size distribution is displayed on a cathode ray screen for photographing, and integrations of selected portions of the display are displayed alphanumerically. The system was calibrated by applying $100 \mathrm{nsec}$ rise and $5 \mu \mathrm{sec}$ decay time voltage pulses from a Hamner $\mathrm{N}-183$ pulser to the input shown on Fig. 3. Voltage measurements were made. with a Tektronix 7904 oscilloscope and discharge magnitudes calculated as $\mathrm{C} \Delta \mathrm{V}$, where $\mathrm{C}$ is $1000 \mathrm{pfd}$ (Fig. 3). The calibration curves used are shown in Fig. 4, where it is seen that the maximum sensitivity is about 1 pcoul. Typical maximum background discharges in the system range from about 5 pcoul at $20 \mathrm{kV}$ to 20 pcoul at $35 \mathrm{kV}$.

The voltage source was a Hipotronics Model 775-10/8117 0-75 $\mathrm{kV}$ power supply with a corona spec of less than 1 pcoul to full voltage.

Since, as we shall show, treeing initiation depends on both the voltage and time of application of voltage we have set up a standard schedule to be used for evaluating the influence of additives. This consists of 10 minute steps of voltage beginning at $5 \mathrm{kV}$ and increasing by $3 \mathrm{kV}$ until tree initiation occurs. Two pulse spectra of 200 seconds duration each are taken during 


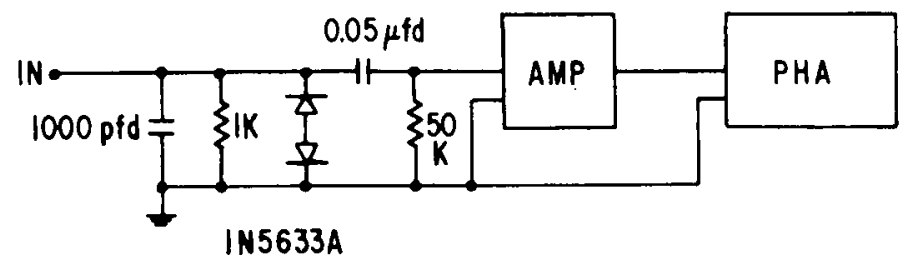

Fig. 3. Electronic circuit and equipment used in discharge detection and measurement.

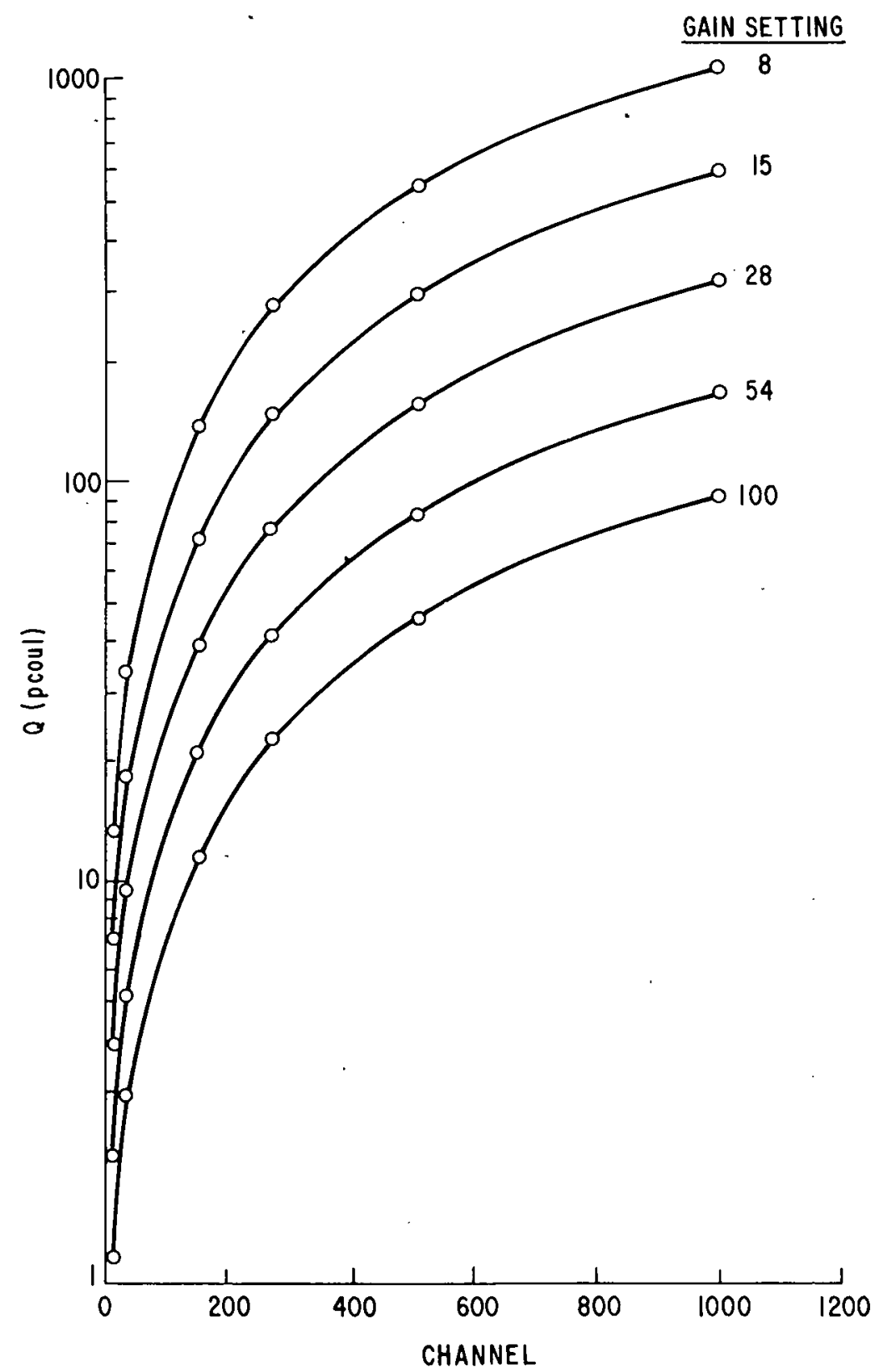

Fig. 4 Calibration curves of charge versus channel number on the pulse height analyser:. The different gain settings on the amplifier are indicated on the figure. Channel 1024 corresponds to $8 \mathrm{~V}$. 
each voltage step. The 1024 channels are divided into five groups and integrals of the total counts in each group are obtained from the analyzer and recorded. In addition, the maximum channel recording discharge pulses is also recorded. Each spectrum is recorded photographically, (for example, see Fig. 8).

In general, when treeing preceded failure, no difficulty was encountered in detecting treeing onset by the sudden large increase in the size of the maximum discharges. Quantitative data relating tree length to maximum discharge size will be reported below.

\subsection{Experimental Results}

4.3.1 Reproducibility of Results

As discussed above three techniques for preparing standard samples were investigated: (1) needle insertion without heating, (2) insertion with heating, and (3) molding of the needle into the sample during compression molding. In the first case tree initiation voltages were very erratic and generally averaged less than one-half those obtained using the other techniques. Often a void at the needle tip could be seen and there is little doubt that the low results could be attributed to the presence of discharges preceding tree initiation.

The results using the other two techniques were found to be essentially identical, as shown in Table 4-1. It is noteworthy that in these, as in all samples, if treeing initiated at or above $26 \mathrm{kV}$, treeing and failure occurred essentially simultaneously, i.e., within a few seconds. If treeing began below $26 \mathrm{kV}$ an increase in voltage usually was required for failure using our schedule. The results of Table 4-1 are plotted on normal probability paper in Fig. 5 where it is clear that in addition to following a normal distribution the data do not distinguish the two techniques for needle incorporation. 


\section{TABLE $\quad 4-1$}

\section{Treeing and Failure Voltages in Polyethylene}

$\begin{array}{rr}\text { Sample } & \text { Method } \\ 1 & \text { IH } \\ 2 & \text { IH } \\ 3 & \text { IH } \\ 4 & \text { IH } \\ 5 & \text { IH } \\ 6 & \text { M } \\ 7 & \text { M } \\ 8 & \text { M } \\ 80 & \text { M } \\ 81 & \text { M } \\ 82 & M \\ 83 & M \\ 84 & M \\ 85 & M \\ 99 & M \\ 101 & M\end{array}$

\begin{tabular}{cc}
$\begin{array}{c}\text { Treeing voltage } \\
(\mathrm{kV})\end{array}$ & $\begin{array}{c}\text { Failure Voltage } \\
(\mathrm{kV})\end{array}$ \\
\cline { 1 - 1 } 26 & 26 \\
29 & 29 \\
17 & 20 \\
32 & 32 \\
26 & 26 \\
32 & 32 \\
26 & - \\
26 & 26 \\
29 & 29 \\
26 & 26 \\
23 & 26 \\
20 & 23 \\
26 & 26 \\
20 & 23 \\
23 & 26 \\
26 & 26
\end{tabular}

TInsertion with heating.

**Molded

Gap $=0.100$ inch

508 treeing voltage $=25.5 \mathrm{kV}$

Needle Radius $=1.5 \mathrm{mil}$

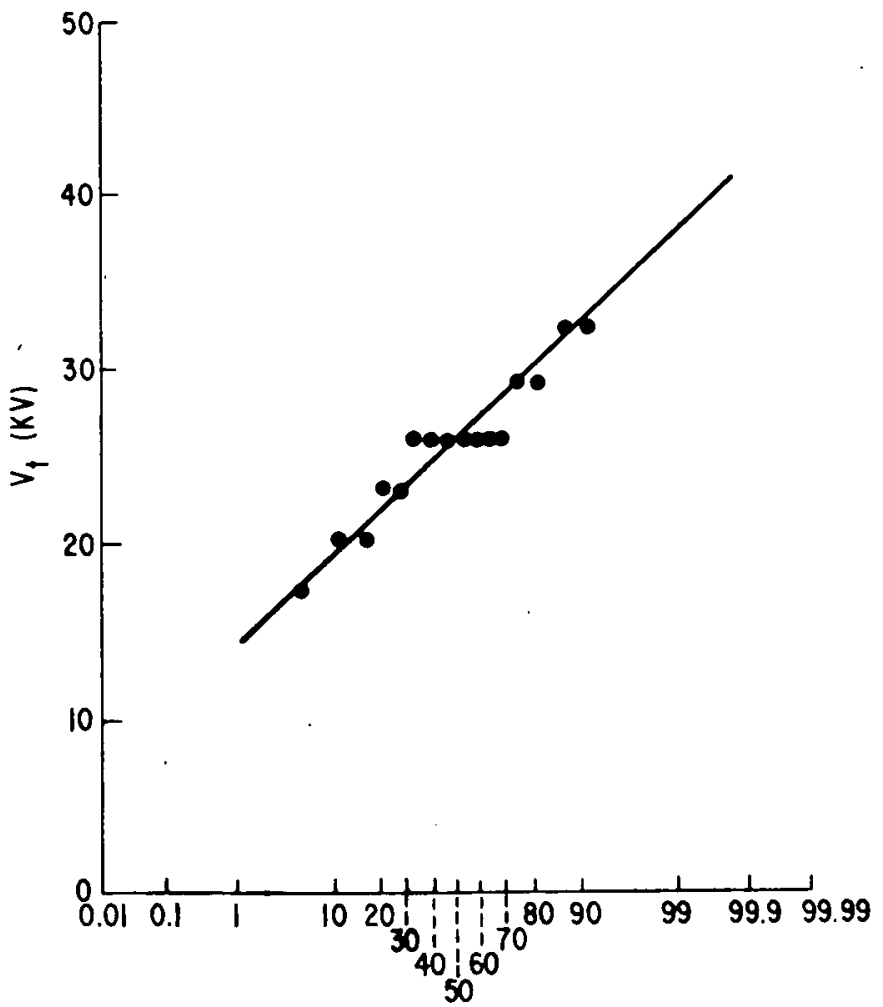

Fig. 5 Normal probability distribution plot of the tree inception voltages in polyethylene. Needle radius $=1.5 \mathrm{mil}$. Gap $=100 \mathrm{mil}$. 


\subsubsection{Tree Inception Voltage and Geometry}

The dependence of tree inception voltage and breakdown voltage on parameters such as gap and needle radius is of interest for two reasons: (1) as a general measure of the sensitivity of our method to variations in these parameters and (2) to supply us with information on the stress at which treeing initiates. The dependence of both tree. initiation voltage, $v_{t}$, and breakdown voltage, $v_{b}$, on gap, using our standard time schedule is shown in Fig. 6. The data at $60 \mathrm{mil}$ is an average of six points while those at $150 \mathrm{mil}$ are an average of four points. Those for $100 \mathrm{mil}$ are taken from Fig. 5. It may be noted that treeing and breakdown are essentially concurrent for the smallest gap, while never concurrent for the largest. As noted above, for $100 \mathrm{mil}$ only those treeing below $26 \mathrm{kV}$ require higher voltages for breakdown. In Table 4-2 we have calculated the stress at the point for which treeing initiates assuming the point may be represented by a hyperboloid and using the formula given by Mason (24).

$$
E=\frac{2 V}{r \ln \left(1+\frac{4 d}{r}\right)}
$$

valid for $d / r>10$. Here $r$ is the radius of curvature of the point and $d$ is the gap. Of course, it is assumed that no field grading occurs in this case. We show peak values of stress, since breakdown on the peak of the applied voltage is expected.

The dependence of treeing voltage (RMS) on needle radius is shown in Fig. 7. Here each point represents a single experiment. Also plotted are the $V_{t}$, calculated from Eq. (4-I) using the average value for the initiation peak stress of $3.15 \times 10^{6} \mathrm{volt} /$ cm (from Table (4-2)). The curvature expected from Eq. (4-1), if initiation occurs at a fixed stress, seems to be observed in Fig. 7. The actual peak stresses calculated for each point in Fig. 7 using Eq. (4-1) are given in Table (4-2). Since the $3 \mathrm{kV}$ steps used in the measurements represent between 10 and $30 \%$ of the tree inception voltage, the differences in tree initiation stress shown in the table are probably not significant. 


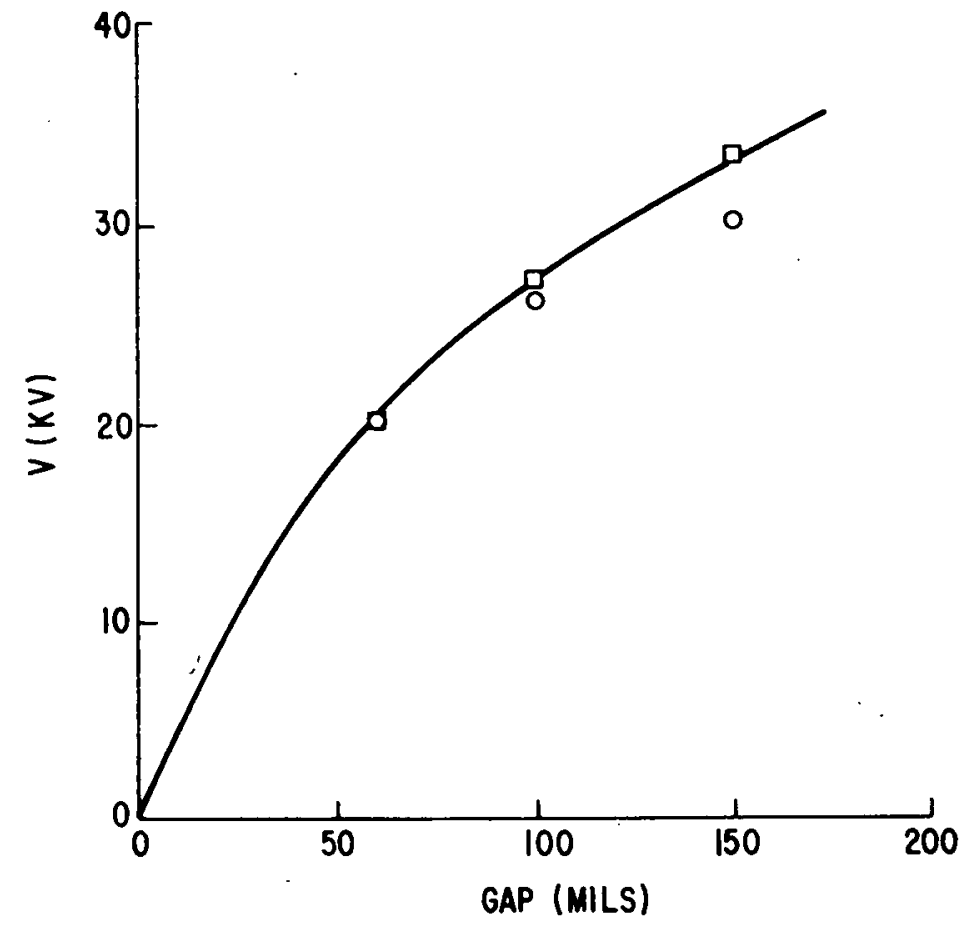

Fig. 6 Dependence of tree inception (0) and breakdown ( $\square$ ) voltages on gap in polyethylene. Needle radius $=1.5 \mathrm{mil}$.

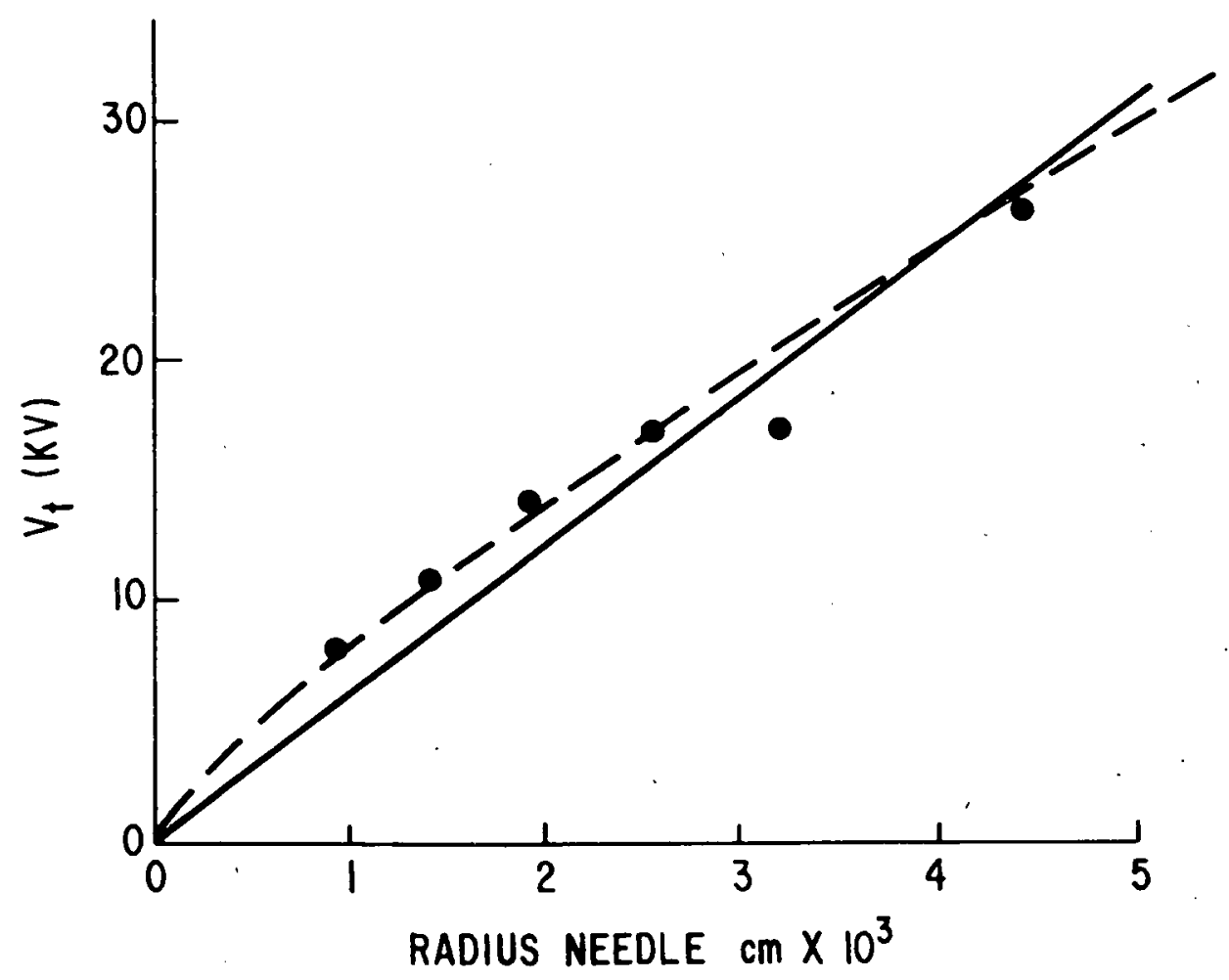

Fig. 7 Tree inception voltage dependence on needle radius in polyethylene. $\mathrm{Gap}=100 \mathrm{mil}$. Dashed line calculated with Eq. (4-1) as described in the text. 
TABLE $\quad 4-2$

Calculated Treeing Stress in Polyethylene

\begin{tabular}{|c|c|c|}
\hline $\begin{array}{r}\text { Gap } \\
\text { (mil) } \\
\end{array}$ & $\begin{array}{l}\text { Point Radius } \\
\left(\mathrm{cm} \times 10^{3}\right) \\
\end{array}$ & $\begin{array}{c}\text { Initiation Stress } \\
\text { Mv/cm Peak }\end{array}$ \\
\hline 50 & 4.45 & 2.58 \\
\hline 100 & 4.45 & 2.99 \\
\hline 150 & 4.45 & 3.27 \\
\hline 100 & 0.95 & 3.40 \\
\hline 100 & 1.27 & 3.66 \\
\hline 100 & 1.91 & 3.31 \\
\hline 100 & 2.54 & 3.16 \\
\hline 100 & 3.18 & 2.62 \\
\hline
\end{tabular}

The "intrinsic strength" of polyethylene, as measured in thin samples with pulsed or DC voltages is usually found to lie between 6 and $9 \mathrm{MV} / \mathrm{cm}$ at room temperature. (25) If grading were occurring (in the absence of additives, here) the values in Table 4-2 would be expected to be higher than the intrinsic strength. One wonders whether, under AC voltages, injected space charge may result in stresses corresponding more nearly to peak-to-peak applied voltages. Alternatively one may speculate that asperities on the points may produce locally higher fields than those calculated, though it is difficult to rationalize why these should be more effective than those normally encountered in experiments using larger electrodes.

\subsubsection{Tree Propagation}

In this section we shall discuss the dependence of tree propagation on voltage and time of application of voltage. In addition, we shall attempt to relate the spectra of discharges to tree size.

We have already commented in Sec. 4.3.1 on the fact that with no void present at the tip of the needle, tree initiation is often concomitant with failure, or at the least, the initial tree may occupy an appreciable fraction of the gap. In order to extend our ability to follow tree propagation we have utilized in some 
of the work in this section, samples intentionally containing voids at the needle tip. These could easily be produced by insertion of reedles without heating, although their size was difficult to control; and they generally ranged from 1 to $5 \mathrm{mils}$ in length.

Typical results for tree propagation with steps of increasing voltage are shown in Fig. 8. Photographs of the growing trees show the applied voltage on the left and time below. The corresponding photographs of discharge spectra show the gain setting below from which discharge magnitudes may be determined using the calibration curves of Fig. 4. The actual tree growth determined from the photographs (the gap is .100 inch) is plotted in the graph shown. In this case a void was initially present.

No visual sign of trees are observed below $18 \mathrm{kV}$ at which point the bush-like tree develops more or less immediately to a diameter of about $23 \mathrm{mil}$. Only spherical, bush-like trees have been observed in polyethylene, while in PVAP the trees are always thin with relatively few branches on a main trunk. In PMMA. both types of trees have been observed. The appearance of the trees coincides unmistakably with the onset of large discharges (note the change in gain at $18 \mathrm{kV}$ ). With time at $18 \mathrm{kV}$, the tree grows in diameter (length) at a deccelerating rate. During this period, the maximum discharge.size also increases. With an increase to. $19 \mathrm{kV}$, the initial rate of growth increases and again decays. Finally, at $20 \mathrm{kV}$ the rate of tree growth increases steadily until failure occurs. This increase in rate before failure usually occurs, but to a variable extent. An examination of the photograph at $10100 \mathrm{sec}$ shows (more clearly in the original photograph) the development of diffuse streamer-like paths crossing between the end of the bush and ground electrode. These are generally observed just prior to breakdown.

In Fig. 9 we show an example of tree initiation and tree propagation to failure occurring at only one applied voltage. No void was initially present. This underscores the importance of establishing a fixed schedule of time and voltage in evaluating 


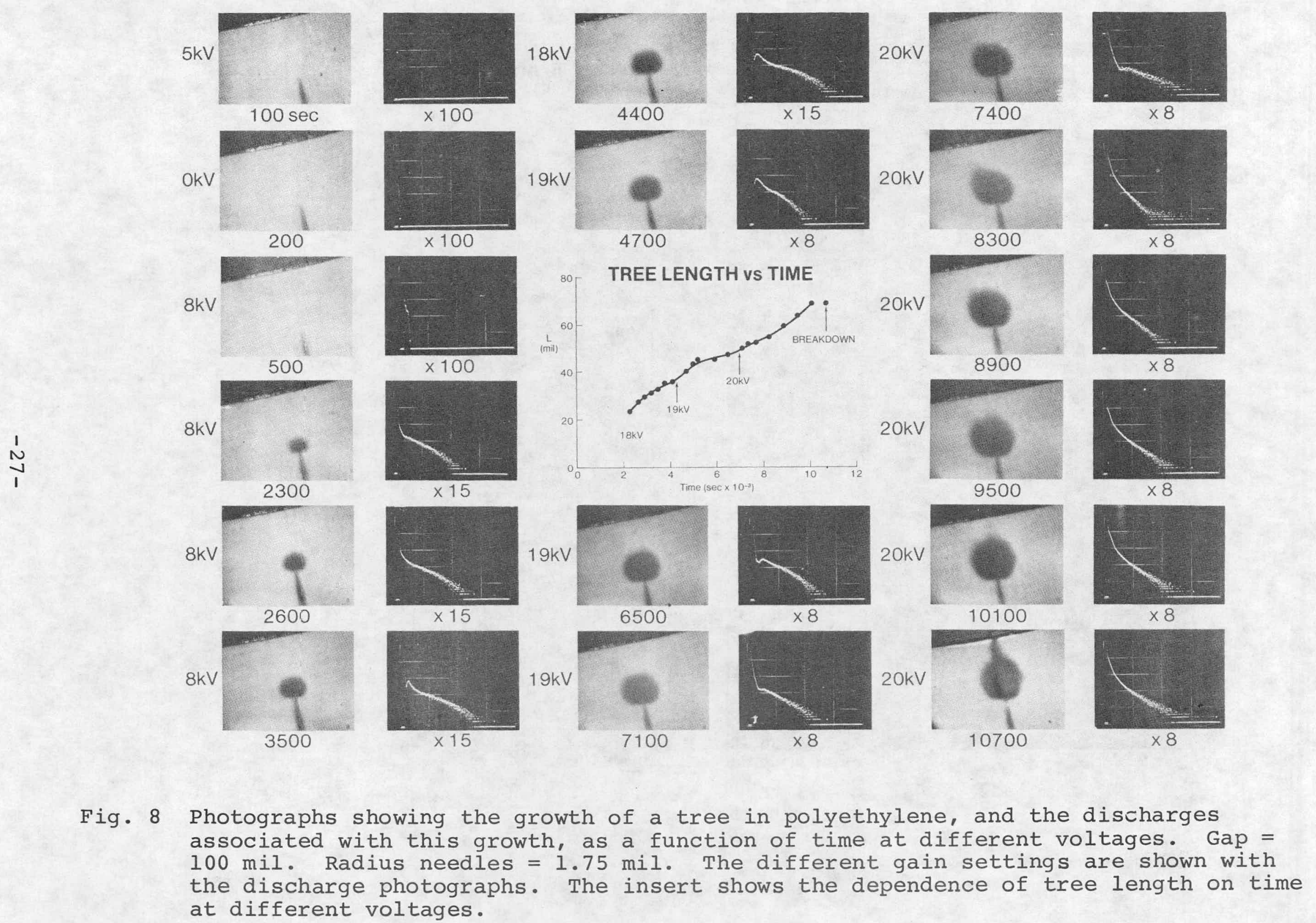




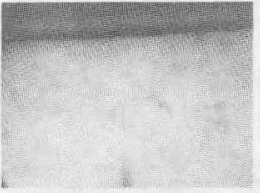

O sec

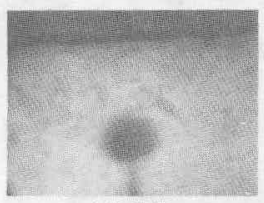

4000

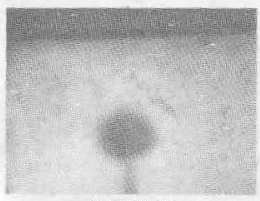

4200

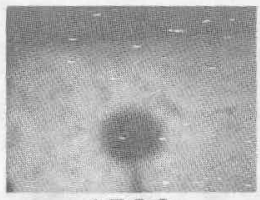

4500

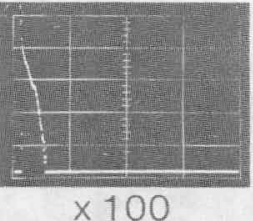

$\times 100$

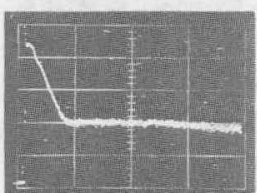

$\times 100$
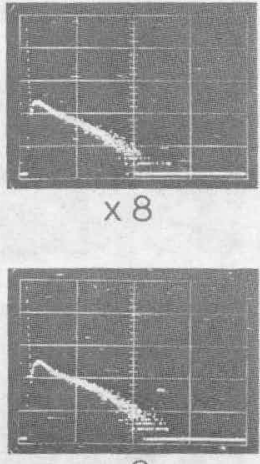

$\times 8$

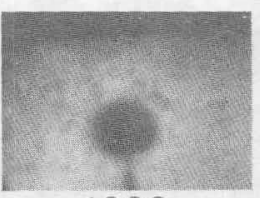

4800

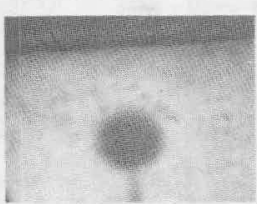

5100

\section{TREE LENGTH vs TIME}

$\mathrm{V}=20 \mathrm{kV}$
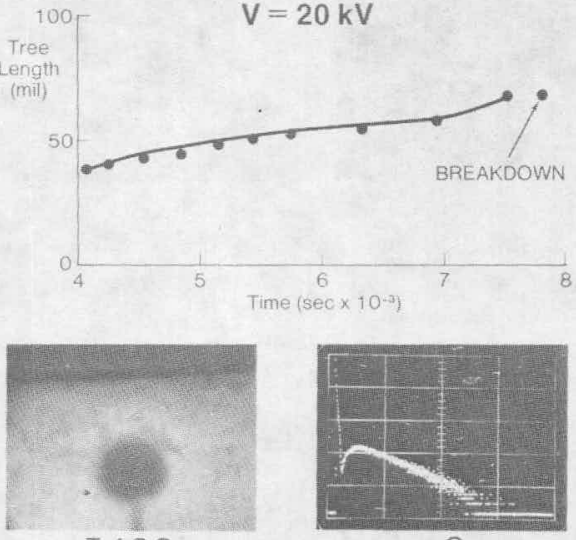

5400

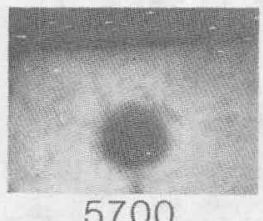

5700

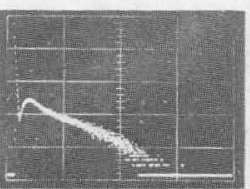

$\times 8$

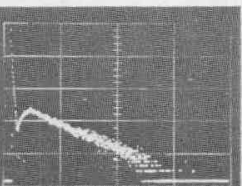

$\times 8$
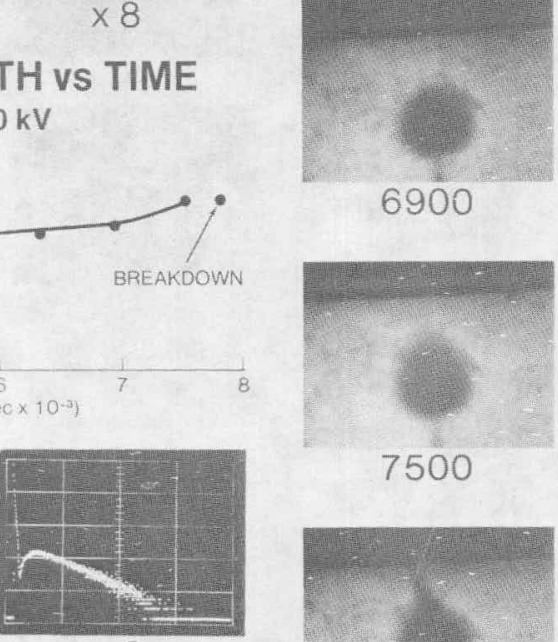

$\times 8$

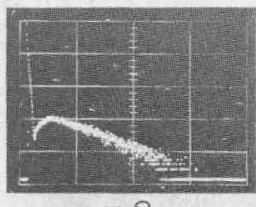

$\times 8$
6900

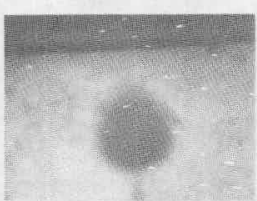

7500

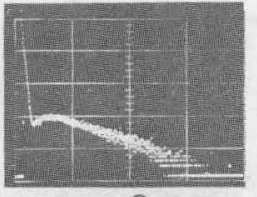

$\times 8$

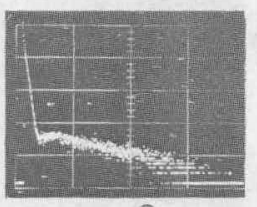

$\times 8$

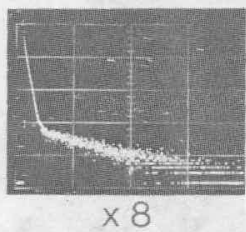

$\times 8$
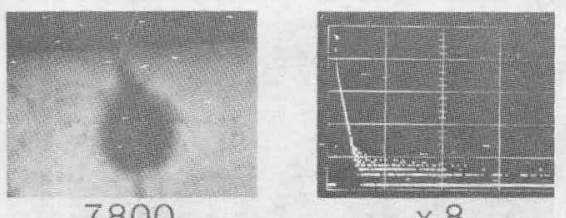

$\times 8$

Fig. 9 Photographs showing tree growth in polyethylene and discharges, as a function of time at $20 \mathrm{kV}$. Gap $=100 \mathrm{mil}$. Needle radius $=1.75 \mathrm{mil}$. The different gain settings are shown with the discharge photographs. Insert shows the dependence of tree length on time at $20 \mathrm{kV}$. 
the role of additives. In this case there is a long period (4000 sec) during which no tree development can be seen, followed by a comparable period during which only very slow growth occurs, and finally, by a short period of increasing growth rate prior to breakdown.

of practical interest from the point of view of predicting the extent of tree growth in opaque filled polyethylene cables it was interesting to correlate discharge magnitudes with tree lengths or diameters. Maximum discharge magnitudes of between 1000 and 2000 pcoul suggested that an appreciable fraction of the whole spherical bush must be discharged. With this in mind we postulate the following model to relate tree length to discharge magnitude. During the largest discharge the whole sphere becomes essentially a perfect conductor, so that the charge which flows onto the dielectric after a discharge must be approximately: $Q=V \Delta C$ where $\Delta C$ is the change in capacity of the sample caused by making the bush conducting and $V$ is the voltage on the wave at which the discharge occurs, i.e., V peak for the largest discharge. If the tree diameter is large compared with the diameter of the point, then $\Delta C$ is very nearly the capacitance between a sphere and plane. This is given by smythe ${ }^{(26)}$ as

$$
\Delta C=\varepsilon a\left[1+\frac{a}{2 d}+\frac{a^{2}}{\left(4 d^{2}-a^{2}\right)}+\ldots\right]
$$

where $a$ is the radius of the sphere and $d$ is the distance between its center and the plane. To the extent that terms of the series on the right after the first may be neglected, a plot of $Q_{\max } / \mathrm{V}$ against tree length should produce a straight line if our assumption that the sphere is conducting is correct. Such a plot is shown in Fig. 10 for a number of measurements involving needles of various radii. As expected, the results are independent of needle radius. Points calculated from Eq. (4-2) using the additional terms shown (further terms add less than $5 \%$ in the range of interest) are included in Fig. 10, and except for the greatest tree length agree with measurements within experimental error. The calculations were fitted to experiment for a $40 \mathrm{mil}$ tree 


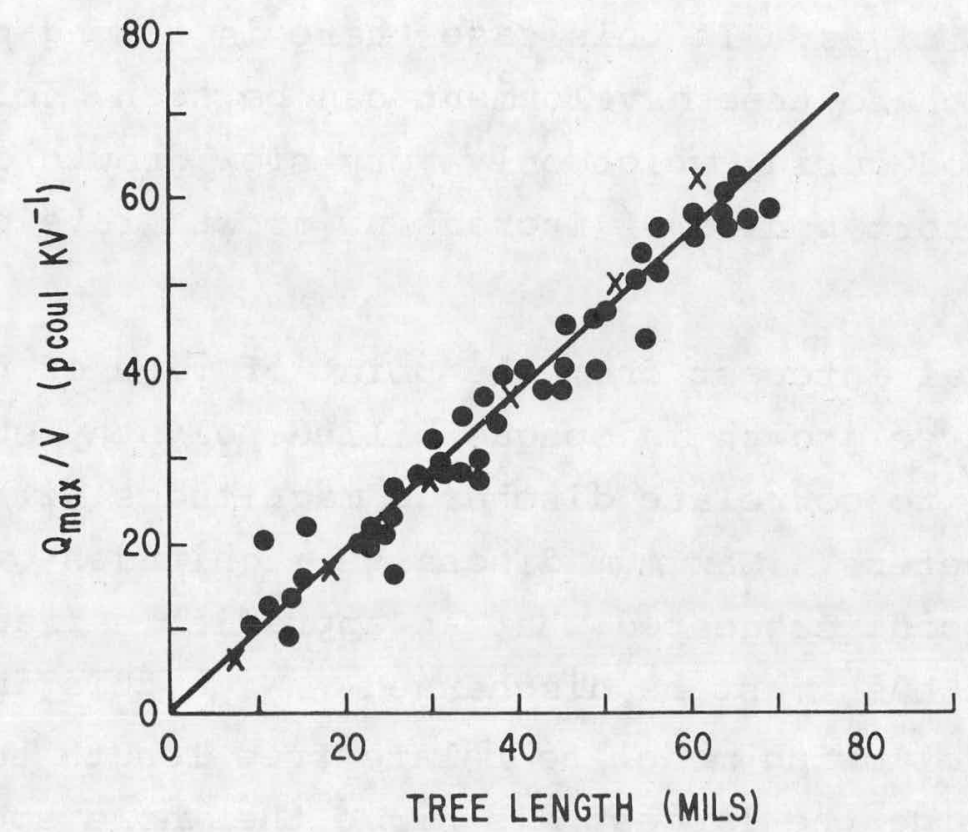

Fig. 10 Relationship between $Q_{\max } / \mathrm{V}$ and tree length in polyethylene. Gap $=100 \mathrm{mil}$. Five different needle radii were used: $0.375,0.50,0.75,1.00$ and $1.25 \mathrm{mil}$. Due to overlap of data we have used only one symbol in the figure. Points calculated with Eq. (4-2) as described in the text, are shown by crosses.

length. $Q_{\max }$ is obtained by linearly extrapolating the spectral tails from photographs such as shown in Figs. 8 and 9. A comparison of the slope of the line of $\mathrm{Fig} .10(0.26 \mathrm{pcoul} / \mathrm{Vcm}$, if peak voltages are used) with that calculated from Eq. (4-2) (2.4 pcoul/ $\mathrm{Vcm}$ ) suggests that in fact only about $10 \%$ of the volume of the sphere is involved in the discharging process. This is consistent with a dissection of this region which shows it to consist of a spongy mass containing many fine tubules. It must be remembered that Eq. (4-2) has been derived for the case of an infinite plane. In our case one dimension of the plane is approximately equal to that of the gap so that Eq. (4-2) should only be regarded as a first approximation.

Figure 11 shows similar plots for samples in which the gap has been varied. The slopes of these lines clearly depend somewhat on the gap used; in fact, they vary nearly inversely with gap. Eq. (4-2) predicts a dependence on gap (inversely) only through second and higher order terms. Thus, while the direction 


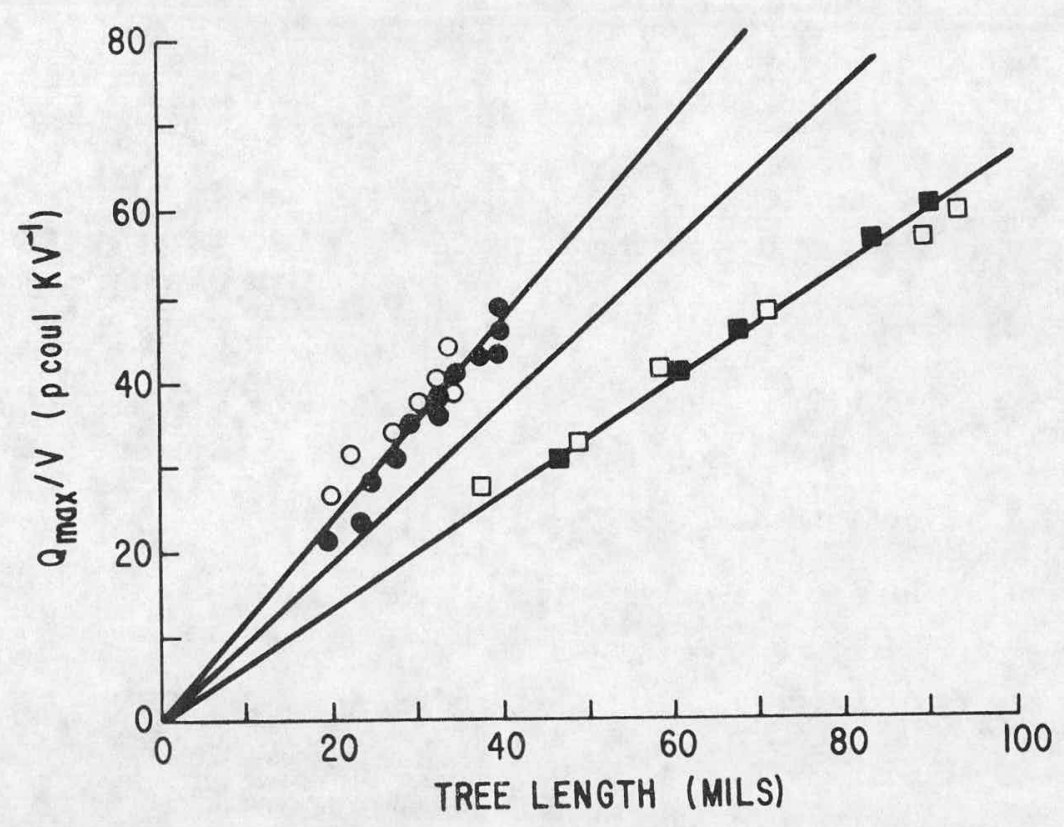

Fig. 11 Relationship between $Q_{\max } / \mathrm{V}$ and tree length in polyethylene for three different gaps. Needle radius = $1.75 \mathrm{mil}$. The open and closed symbols refer to different samples - $(0,0) 60 \mathrm{mil}$ gap, ( $(\square, 0) 150 \mathrm{mil}$ gap. The middle solid line is that of Fig. 10 for $100 \mathrm{mil} g a p$.

is as predicted the magnitude of the effect observed is somewhat greater. This suggests that the fractional volume of the growing bush involved in the discharges is larger with smaller gaps.

\subsubsection{Breakdown Patterns}

one of the most striking and consistent observations we have made is that final failure in polyethylene always occurs when the growing tree has crossed a constant fraction of the gap--about $60 \%$. This is shown, for example, in the upper photographs in Fig. 12 for three different gaps, where the extent of bush growth before development of the final breakdown path is evident. This result offers further evidence that during discharges in the bush it is an essentially conducting sphere. Thus, for a small bush, the maximum field at its surface decreases with increasing size for a fixed voltage, because the radius of curvature is increasing. Beyond a certain size the field begins to increase since the spacing between sphere and ground is decreasing. Therefore, if the tree reaches this size, further growth will occur without 

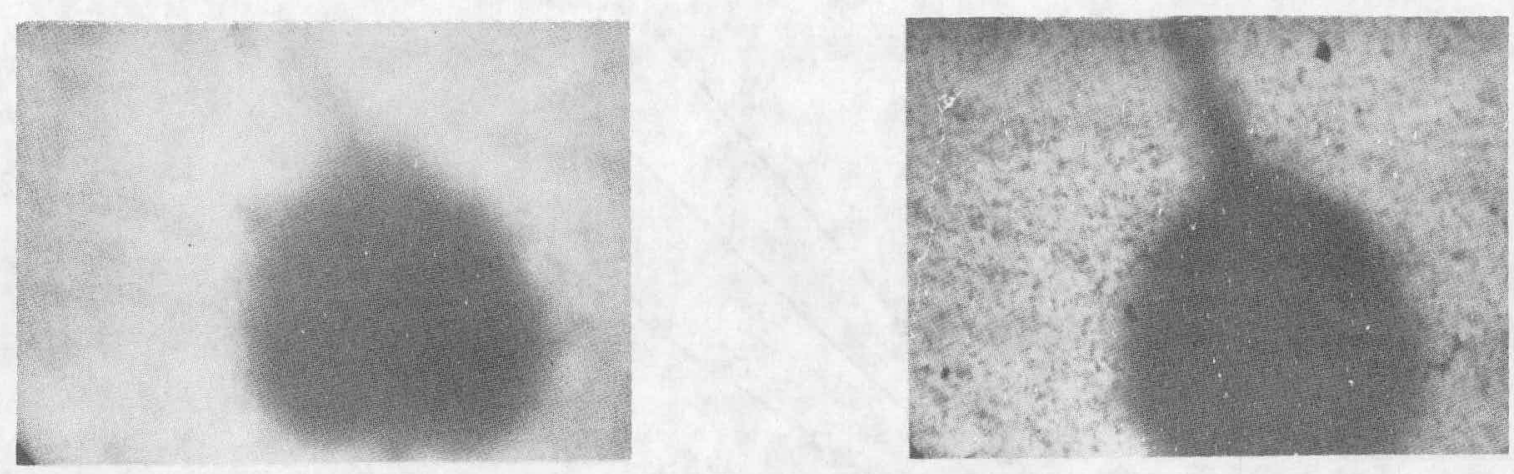

\section{PE -150 mils}
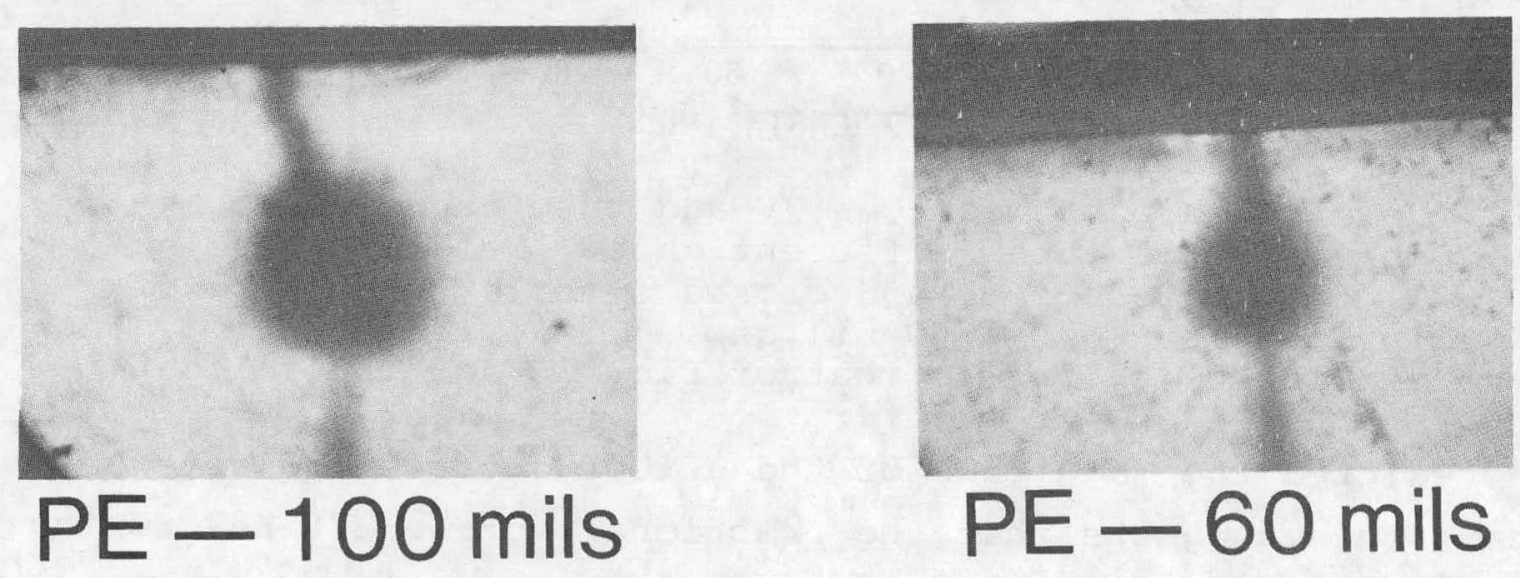

\section{$P E-60$ mils}
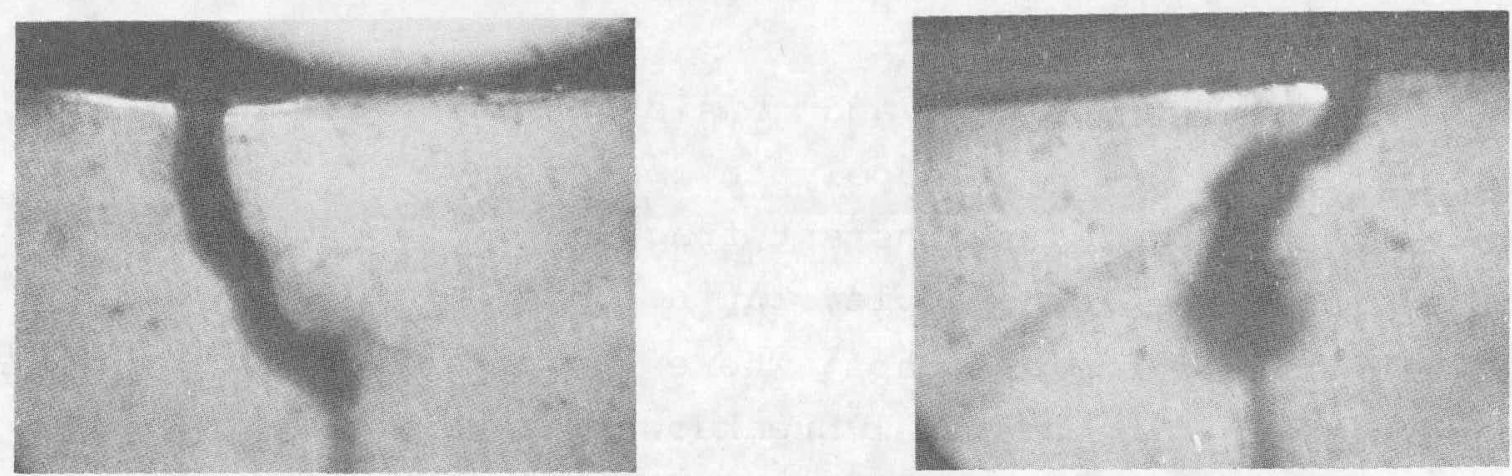

\section{$P E-5 \%$ L25352E - 100 mils}

Fig. 12 Breakdown patterns in polyethylene for different gaps. For the $150 \mathrm{mil}$ gap the left picture was taken $20 \mathrm{sec}$. before breakdown. The bottom two pictures illustrate the effect of $\mathrm{L} 25352 \mathrm{E}$ on tree formation and breakdown patterns. 
a further increase in voltage and the tree will become unstable. For concentric sphere geometry, it can be easily shown that this occurs half way across the gap. For sphere to plane geometry, such as shown in our photographs (Figs. 8 and 9), a slightly larger growth for instability would be expected. Thus; our observation of breakdown with tree growth of $60 \%$ of the gap seems reasonable. These considerations also explain why if tree initiation occurs above a certain critical voltage failure occurs essentially simultaneously with initiation.

The first photograph in Fig. 12, taken 20 seconds before the failure shown in the second photograph, shows clearly the prebreakdown. streamer alluded to above. The last two photographs are two examples of failure in polyethelyene containing an ionic additive, Lubrizol L25352E (see Part II, Section 4). Marked suppression of treeing is evident from the fact that in both cases failure occurred well before the bushes developed $60 \%$ across the gap. As will be seen in Part II, the failure voltage is somewhat above that for pure polyethylene, so that it may be concluded that the Lubrizol truly suppresses treeing rather than merely increasing the breakdown voltage.

\subsubsection{Summary of Results}

The major results described in this section may be summarized as follows:

1. Satisfactory techniques have been developed for treeing evaluation.

2. With molded needles, treeing initiates on the average, at a critical electrical stress which is nearly independent of needle radius or gap over the range investigated. In polyethylene this stress is very nearly one-half of the intrinsic strength normally measured with DC or pulsed DC. With voids present, treeing initiates at a considerably lower stress.

4. In polyethylene only bushlike trees are observed. During discharges, these appear to be conducting. 
5. Relatively simple correlations between tree size and maximum discharge size, obtained from pulse height analysis, are found, suggesting that this technique would be useful for examining "dry" tree growth in cables. A model has been developed for relating discharge size to tree length.

6. Final failure in polyethylene occurs when the tree has grown across $60 \%$ of the gap. This is consistent with the model described. 


\section{REFERENCES}

1. W. Vahlstrom, Trans. IEEE PAS-91, 1023 (1972).

2. J. H. Mason, Proc. IEE 98, 44 (1951).

3. J. H. Mason, Proc. IEE 102-C, 254-263 (1955).

4. D. W. Kitchen and 0 . S. Pratt, Trans. AIEE Pt 3 (PAS) $\underline{77}$, 180-186 (1958).

5. J. H. Mason, Ch. in Vol. 1 of Progress in Dielectrics, Heywood \& Co., London, England, 1959.

6. T. Miyashita and T. Inoue, 9th Electrical Insulation Conference, 1969, pp. 131-135.

7. T. Miyashita and T. Inoue, Electrical Engineering in Japan 87, 76-83 (1969).

8. T. Tabata, T. Fukuda, and Z. Iwata, Trans. IEEE PAS-91, 1361 (1972).

9. T. Tabata, H. Nagai, T. Fukuda, and Z. Iwata, Trans. IEEE PAS-91, 1354. (1972).

10. "Treeing Phenomena in Organic Insulating Materials," Japanese IEE Study Committee Technical Report 100, 1971 (In Japanese).

11. 1972 Annual Report of the Conference on Electrical Insulation and Dielectric Phenomena, National Academy of Sciences, Washington, D.C., 1973.

12. 1973 Annual Report of the Conference on Electrical Insulation and Dielectric Phenomena, National Academy of Sciences, Washington, D.C., 1974 .

13. 1974 Annual Report of the Conference on Electrical Insulation and Dielectric Phenomena, National Academy of Sciences, Washington, D.C., 1975 .

14. S. Fujiki, H. Furusawa, T. Kuhara, and H. Matsuba, Trans. IEEE PAS-90, 2703-2709 (1971). 
15. D. W. Kitchen and O. S. Pratt, Trans. AIEE, Pt. 3 (PAS), 112-121 (1962).

16. 0. S. Pratt, pp. 74-79, 1965 Annual Report of the Conference on Electrical Insulation and Dielectric Phenomena, NAS-NRC Publ. 1356, Washington, D. C., 1966.

17. E. J. McMahon and J. R. Perkins, Trans. IEEE PAS-83, 1253-1261 (1964).

18. J. W. Billing and J. H. Mason, pp. 93-96, Conference on Dielectrics Materials; Measurements, and Applications 20-24 July 1970, University of Lancaster, England, Conference Publication No. 67 IEE, London, England."

19. T. Hayami, Trans. IEEE PAS-88, 897-904 (1969).

20. T. Miyashita, T. Owada, and O. Kamata, Hitachi Review, 20, 174-180 (1971).

21. T. Miyashita and T. Inoue, J. IEEE Japan 87, 19-21 (1967) .

22. M. Wien, Phys. Zeits, 32 , 545 (1931):

23. L. Onsager, J. Chem. Phys. $\underline{2}$, 599-615 (1934).

24. Ref. 5, p. 17 .

25. P. Fischer, 1974 Annual Report of the Conference on Electrical Insulation and Dielectric Phenomena, National Academy of Sciences, Downingtown, Pa. pp. 661-670.

26. W. R. Smythe, Static and Dynamic Electricity, McGraw-Hill Book Company, Inc., New York, Second Edition, 1950, p. 121 .

27. J. Crank and J. S. Park, Diffusion in Polymers, Academic Press, 1968, p. 137 .

28. Ibid, p. 87 .

29. R. M. Fuoss and F. Accasina, Electrolyte Conductance; Interscience Publishers, Inc., New York, 1959, p. 215.

30. M. Nawata and H. Kawamura, Elect. Eng. Japan, 88, 45 (1968). 


\section{APPENDICES}

Appendix 1. The Second Wien Effect

A weak electrolyte is defined as a material which is only slightly dissociated into ions when dissolved in the solvent of interest.

$$
\mathrm{A}^{+}-\mathrm{B}^{-}+\mathrm{A}^{+}+\mathrm{B}^{-}
$$

Since the coulombic energy holding the ions together in the neutral pair $\mathrm{A}^{+}-\mathrm{B}^{-}$increases with decreasing dielectric constant the equilibrium expressed by Eq. (6-1) will be far to the left in low dielectric solvents such as polyethylene.

If $\mathrm{A}^{+}-\mathrm{B}^{-}$is placed in an electric field there will be a force tending to align the molecule in the field. As a result of this alignment there will be produced a net force tending to pull the molecule apart, thus pushing the equilibrium (6-1) to the right. As a result of the increased concentration of free ions the conductivity will increase. This is called the second Wien effect. The force tending to align the molecules in the field direction is opposed by the randomizing process of Brownian motion, which results from the thermal energy of the molecules. Thus, increasing temperature reduces the effectiveness of the field in promoting dissociation, as is evident in Eq. (3-3).

The mathematical expression of this picture is developed in the paper of onsager. (23) 
Appendix 2. Conductivity and Additive Concentration

Assuming an additive which partly dissociates into singlycharged anions and cations as shown in Eq. (6-1) then the current density, $j$, with an applied field $E$ is given by

$$
j=\text { nev }_{1}+\text { nev }_{2}
$$

where $e$ is the electronic charge, $n$ is the concentration of ions (assumed equal for the two ions) and $v_{1}$ and $v_{2}$ are their respective velocities. Since the velocity is given by

$$
v=\mu E
$$

where $\mu$ is the corresponding ionic mobility,

$$
j=\operatorname{neE}\left[\mu_{1}+\mu_{2}\right]
$$

But by Ohm's Law, the conductivity, $\sigma$, is given by

$$
\begin{aligned}
j & =\sigma E \\
\therefore \sigma & =\operatorname{ne}\left[\mu_{1}+\mu_{2}\right]
\end{aligned}
$$

which expresses the relation between conductivity and ion concentration and mobilities.

Values for ion mobilities are not available, but estimates can be made from measured diffusion constants, $D$, using the wellknown Nernst-Einstein relation

$$
\mu / D=\frac{e}{k T}
$$

where $\mathrm{k}$ is Boltzman's constant, $\mathrm{T}$ is absolute temperature and $\mathrm{kT}$ is expressed in units of electron volts. Thus, at room temperature

$$
\mu / \mathrm{D} \approx 40 \mathrm{eV}^{-1}
$$

From this point, only order of magnitude calculations are possible since specific data on diffusion constants for our systems are not available. For small molecules such as $\mathrm{N}_{2}, \mathrm{O}_{2}$ ' $\mathrm{CO}_{2}$, and $\mathrm{CH}_{4} \mathrm{Crank}$ and Park ${ }^{(27)}$ give values for $\mathrm{D}$ at $25^{\circ} \mathrm{C}$ for 
low density polyethylene ranging from $2-5 \times 10^{-7} \mathrm{~cm}^{2} \mathrm{sec}^{-1}$. In another investigation ${ }^{(28)}$ in natural rubber, D decreased by a factor of about $\mathrm{six}$ in going from methane $\left(\mathrm{CH}_{4}\right)$ to butane $\mathrm{C}_{4} \mathrm{H}_{10}$. It appears likely from these results that for our additives $D$ will be greater than $10^{-10} \mathrm{~cm}^{2} \mathrm{sec}^{-1}$. Let us assume, then, from Eq. (6-8) that $\mu_{1}+\mu_{2}>10^{-9} \mathrm{~cm}^{2} \mathrm{volt}^{-1} \mathrm{sec}^{-1}$.

Using this value we find from Eq. $(6-6)$ with $\sigma=10^{-15}$ (sect. III - low fields)

$$
\mathrm{n} \leq \frac{\sigma}{\mathrm{e}\left[\mu_{1}+\mu_{2}\right]}=\frac{10^{-15}}{i .6 \times 10^{-19} \times 10^{-9}}=6 \times 10^{12} \text { ion } \mathrm{cm}^{-3}
$$

When one realizes that there are approximately $4 \times 10^{22}$ atoms $\mathrm{cm}^{-3}$ in polyethylene it is clear that the required ion concentration is very small. However, let us now examine the required additive concentration in view of the expected small degree of dissociation in the low dielectric constant polymer.

The dissociation constant is defined as

$$
K_{D}=\frac{\left[\mathrm{A}^{+}\right]\left[\mathrm{B}^{-}\right]}{[\mathrm{AB}]}=\frac{\left[\mathrm{A}^{+}\right]}{[\mathrm{AB}]}
$$

where $\left[\mathrm{A}^{+}\right]$and $[\mathrm{AB}]$ refer to the concentrations of $\mathrm{A}^{+}$and $\mathrm{AB}$ usually measured in mole liter ${ }^{-1}$. ( $[A B]$ is the required additive concentration, i.e., the concentration to give sufficient conductivity for stress grading.) The dependence of $\mathrm{K}_{\mathrm{D}}$ on dielectric constant is illustrated in Table 6-1 measured for a quaternary ammonium salt in a mixed solvent with varying dielectric constant $(\varepsilon) .(29)$ while the precise values of $K_{D}$ will depend to some extent on the nature of the ions and the nonelectrostatic interactions with the medium, the order of magnitude and the effect of dielectric constant are expected to be correct. Using this table we can obtain an estimate of the concentration of additives needed to obtain the ion concentration, $n$, calculated above. Thus 
TABLE 6-1

Dissociation Constants for Tetra-iso-Amylammonium Nitrate in Dioxane-Water Mixtures

$\begin{array}{lr}\frac{8 \mathrm{H}_{2} \mathrm{O}}{0.60} & \frac{\varepsilon}{2.38} \\ 1.24 & 2.56 \\ 2.35 & 2.90 \\ 4.01 & 3.48 \\ 6.37 & 4.42 \\ 9.50 & 5.84 \\ 14.95 & 8.5 \\ 20.2 & 11.9 \\ 53.0 & 38.0 \\ \mathrm{n}=\frac{\left[\mathrm{A}^{+}\right] \mathrm{x} N}{1000} & \end{array}$

\begin{tabular}{ll} 
& \multicolumn{2}{l}{$\mathrm{K}_{\mathrm{D}}$} \\
2 & $\times 10^{-16}$ \\
1 & $\times 10^{-14}$ \\
1 & $\times 10^{-12}$ \\
2.5 & $\times 10^{-10}$ \\
3.0 & $\times 10^{-8}$ \\
$1.65 \times 10^{-6}$ & $\times 10^{-4}$ \\
$1.00 \times 10^{-4}$ \\
$9.0 \times 1$ \\
0.25
\end{tabular}

where $\mathrm{N}$ is Avogadro's number $=6.02 \times 10^{23}$.

From Eqs. $(6-9)$ and $(6-10)$

$$
[A B]=\frac{n^{2} \times 10^{6}}{N^{2} K_{D}} \text { mole liter }{ }^{-1}
$$

Expressed as percent by weight

$$
8 A B=\frac{n^{2} \times 10^{6} \times 333 \times 100}{N^{2} K_{D} \times 1000}=\frac{n^{2} \times 10^{8}}{3 N^{2} K D}
$$

where the molecular weight of tetrabutyl ammonium fluoroborate is 333 and we have assumed a density for PE of 1 .

Utilizing Eq. (6-12) and Table 6-1 we calculate the percent additive required vs dielectric constant in Table 6-2. 
TABLE 6-2

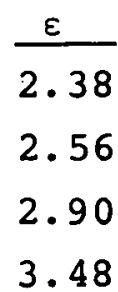

$\stackrel{\circ \mathrm{AB}}{15}$

15

0.3

.003

.00001

It must be realized that these are only order of magnitude calculations since precise data for our system are not available. However, they serve to show two things, (1) that data presently available support the feasibility of the approach and (2) the extreme sensitivity to dielectric constant of the medium, so that modifications in this parameter should serve as a cushion to fit a particular combination of additive properties to the required final conductivity. 
Appendix 3, Heating in the Grading Region

It might be argued that heat generated in the high field region where grading is occurring may lead to thermal runaway. Indeed, if the volume involved in this region is sufficiently large, then this certainly must be true. In this section we give a crude order of magnitude estimate of the temperature rise to be expected in the high field region.

Let us suppose that this region is spherical, of radius $r$ with a field in it of $10^{6}$ volt $\mathrm{cm}^{-1}$. Then, using our estimate of $10^{10} \Omega \mathrm{cm}$ for the required resistivity the current density in the volume will be

$$
j \approx \frac{E}{\rho}=\frac{10^{6}}{10^{10}}=10^{-4} \mathrm{amp} \mathrm{cm}^{-2} .
$$

This corresponds to a heat generation of

$$
W=j E=10^{6} \times 10^{-4}=100 \text { watt } \mathrm{cm}^{-3} \text {. }
$$

The heat flux out of this spherical region will be

$$
Q=\frac{\frac{4}{3} \pi r^{3} \times 100}{4 \pi r^{2}} \approx 30 r \text { watt } \mathrm{cm}^{-2} \text {. }
$$

Assuming a thermal conductivity of $2 \times 10^{-3}$ watt $/{ }^{\circ} \mathrm{C} \mathrm{cm}$, then

$$
\text { grad } \mathrm{T}=\frac{30 \mathrm{r}}{2 \times 10^{-3}}=1.5 \times 10^{4} \mathrm{r}^{\circ} \mathrm{C} / \mathrm{cm}
$$

If $\mathrm{r}=1 \mathrm{mil} \equiv 2.5 \times 10^{-3} \mathrm{~cm}$

$$
\operatorname{grad} \mathrm{T}=38^{\circ} / \mathrm{cm}
$$

or the temperature difference.

$$
\Delta T \approx 2.5 \times 10^{-3} \times 38 \approx 0.1^{\circ}
$$

This computation is admittedly crude but serves to show that excessive temperature rises will not appear over the sorts of grading distances needed for normal small inclusions. 
Appendix 4. Work Statement from original General Electric Company Corporate Research and Development Technical Proposal (CRD:3351.008E) submitted to Electric Power Research Institute in June 1973 on "A New Class of Additives to Inhibit Tree Growth in Solid Extruded Cable Insulation."

Section numbers are those used in original proposal. Only Sections 5.1 and 5.2 were covered in the present work. (Sections 5.3 to 5.7 would need to be completed as a Phase 2 program.) section 5.1 outlined the work required to accomplish the portions of the program financed by in-house GE funds. Section 5.2 describes the work required to complete these portions of the program funded by EPRI and ERDA.

5.1 GE-Funded Work--Develop Techniques for Evaluating Treeing.

\section{1 .1 Test cell}

We would envisage the study of tree growth from needles either molded in or inserted into thick slabs of our materials. Needles will be ground down to a standard radius of curvature in a ball mill with carborundum. This technique has already been used successfully by B. T. McKenzie of our Company's Wire and Cable Department. The samples will probably be immersed in either a silicone or fluorocarbon fluid to prevent surface discharges, and with spacings of 1 to $10 \mathrm{~mm}$ between needle and ground plane, $60 \mathrm{~Hz}$ voltages of 10 to $20 \mathrm{KV}$ will be applied. Based on earlier work, substantial tree growth should be observed in untreated $\mathrm{PE}$ in times ranging from a few minutes to a few hours. The measurements of interest will be length of the tree as a function of time, voltage and temperature.

\section{1 .2 Optical Measurements}

The simplest measurement of tree length is made by removing the needle and microtoming the sample in the neighborhood of the tree. This has the disadvantage of destroying the sample so that a fresh sample must be used for each point on the growth curve. 
For this reason we shall attempt also to measure the length of the growing tree as a function of time by direct observation with a microscope while voltage is applied. This has been done successfully by Nawata and Kawamura $(30)$.

\subsubsection{Discharge Measurements}

For opaque materials, optical techniques, even those involving microtoming, are difficult. Since Nawata and Kawamura (30) have found a simple relation between the tree length and maximum discharge magnitude as measured with a discharge detector, we shall include such measurements. We have available, and will use, a pulse height analyzer for the study of discharges in insulation. We believe that this will add a new dimension to a study of the relationship between tree growth and discharge characteristics.

5.2 EPRI--Funded Work--Evaluation of Prospective Additives.

5.2.1 Establish a Suitable combination for treeing evaluation

5.2.1.1 Initial Materials

We shall start with uncrosslinked polyethylene (PE) as the base polymer because of the ease with which it may be molded and milled. The first additive we shall use will be tetrabutyl ammonium fluoroborate (TBF), because of the large organic groups on the cation which should impart solubility in polyethylene, and because of its good chemical stability.

\subsubsection{Measurement of DC Conductivity}

We shall mill in three concentrations, $0.2,1.0$ and $5.08 \mathrm{TBF}$, and measure the dielectric constant and loss tangent as a function of frequency ( 1 to $10^{4} \mathrm{~Hz}$ ) and temperature $\left(25-100^{\circ} \mathrm{C}\right)$. . Samples will be compression molded sheets 5 - 10 mils in thickness. From the frequency dependence so measured one can separate the contributions of the additives to ionic conductivity (low frequencies) and 
to dipolar losses. This leads to less ambiguous values for the dc conductivity because of problems with polarization currents in dc measurements.

\subsubsection{Field Dependence of DC Conductivity}

Assuming that a suitable dc conductivity can be established above, we shall measure the dependence of this on applied field at $60 \mathrm{~Hz}$ (a) as a check on the fact that our interpretation of the frequency dependence is correct and (b) as a check on our theory that this effect can be used for field grading.

\subsubsection{Solubility of $\mathrm{TBF}$ in $\mathrm{PE}$}

If the conductivity satisfies the requirements for field grading, we shall next proceed to sect. 5.2.2, i.e., treeing studies. At the same time, since only that part of the TBF which is soluble will contribute to the conductivity while that which is not may contribute to a degration in electric strength by forming agglomerates or particles we shall attempt to measure the solubility at this point. This may be accomplished by infrared absorption measurements in our analytical laboratory providing the spectrum of the dissolved material is sufficiently different from the agglomerated material, or it may be accomplished by simply determining at what concentration of added TBF the conductivity becomes independent of further additions.

\subsubsection{Additives to Increase Dielectric Constant}

If the conductivity does not satisfy the requirements for field grading we shall attempt to increase it by increasing the dielectric constant, and thus the degree of dissociation (see Appendix 2) by the addition of a poly chlorinated biphenyl (PCB). Three concentrations, 10, 20 and $30 \%$, will be milled into $\mathrm{PE}$ containing $5 \% \mathrm{TBF}$, and the 
measurements described in sects. 5.2 .1 .2 and 5.2 .1 .3 will be made on these materials.

Assuming that conductivities in the needed range are obtained, we would establish curves of conductivity vs TBF concentration and $\mathrm{PCB}$ concentration in order to minimize the latter, and then proceed to treeing studies.

\subsubsection{Other Ionic Additives}

If at this point we were still unsuccessful in obtaining the desired conductivity we would examine other ionic additives. The most likely candidates would be those containing organic substitutents on the anion. This would increase the degree of dissociation of the ions as well as the solubility.

\subsubsection{Measurement of Additive Effectiveness}

Using all of the measurement techniques discussed above, for control samples of pure polyethylene and for samples containing at least two concentrations of the additive developed in sect. 5.2.1, we shall establish a family of curves relating the rate of tree growth to voltage and temperature. These curves will constitute the first measure of the effectiveness of the additives and of the technique for tree prevention.

\subsubsection{Analyze the System for Use in Cables}

The object here is to predict the range of concentrations of both kinds. of additives which will allow the system to remain tree-resistant over the required temperature and stress range to be used in a cable. We then wish to establish the behavior of other important properties over this concentration, temperature and stress range. These properties include dielectric constant, loss tangent, short-time dielectric strength and mechanical properties. 
Data for the first two of these will be partly established in sects. 5.2.1 and 5.2.2 above, though, since the object was to demonstrate the effect with a minimum of data more will probably be required at this point. Short-time dielectric strength measurements will be made according to ASTM D-149-64 requirements. Tensile measurements will also be made.

At this point we would hope to have determined:

(a) whether our present system could be used over the full range of temperature and stress required in a cable

(b) if not, what properties must be altered in order to make it viable.

\subsection{Examine Other Systems}

Assuming that the analysis carried out in sect. 5.2.3 suggests that improvements in certain properties are desirable or necessary, we shall examine other materials with such improvements in mind. Possibilities include using polymer modifications for increasing dielectric constant, and thus conductivity, rather than liquid additives. For example, polyethylene with varying degrees of chlorosulfonation (Hypalon) should offer dielectric constants ranging from 2.3 to 9 . Other liquid additives such as esters commonly used in plasticizers may offer advantages. The procedures developed in sects. 5.2 .1 and 5.2 .2 will be used to obtain minimum data needed to establish whether an improved system is obtained. At this point an analysis similar to that discussed in sect. 5.2.3 would be carried oult for other prospective cable materials such as XLPE and EPR.

\subsection{Choose Optimum System}

Based on the analysis to date it should be possible to narrow the choices for practical application to one or two. 
5.5 Obtain Accelerated Life Tests on Small Cable Samples With Above-Normal Voltages

Here it is envisaged that we would develop volt-time curves for cable geometries (reduced scale) using standard cable materials such as XLPE and EPR as well as the materials chosen in sect. 5.2.3. It may be of advantage in these tests to include samples containing needles acting as artificial asperities and inclusions. We shall include also an evaluation of the performance of the samples in water. Negative results during this portion of the program may necessitate returning to sect. 5.3 for further development work.

5. 6 Construct Full-Voltage Samples for Standard Factory Testing

5.7 Construct Suitable Lengths of Cable for Life Testing at Waltz Mill

As has already been suggested above, we believe that the complete program outlined above should be conducted in two phases. The first phase, which it is proposed should be carried out during the first year, would include those items in the program labelled 4.1 and 4.2 according to the work statement sects. 5.1 and 5.2. At the end of this time we should have developed sufficient in-. formation so that a thorough and complete work statement can be developed for the second half of the program which would then be negotiated as phase 2. We consider the work statement outlined between sects. 5.3 and 5.7 as preliminary and only indicative of the methods we might employ and the direction in which we might go. 


\section{PART II}

Program funded jointly by the Electric Power

Research Institute and the Energy Research and Development Administration

DEVELOPMENT OF ADDITIVES TO INHIBIT TREE GROWTH IN SOLID EXTRUDED CABLE INSULATION 


\section{INTRODUCTION}

The desirability of inhibiting tree growth in solid extruded cable dielectrics in order to achieve improved performance and service life of solid extruded cables was identified in sections I and II of Part 1. Part 1 described the development of experimental techniques to study tree initiation and propagation. Part II, the program funded jointly by EPRI and ERDA, will cover the development of additives to inhibit treeing in solid extruded cable dielectrics.

The work described in Part II has ranged beyond the scope of the original Work statement and we believe we have identified many factors which should be of help in future studies of treeing and in the development of additives to inhibit treeing. Promising additives have been identified and attempts have been made to understand the mechanisms involved. Before proceeding with a detailed account of this work, we shall summarize our findings in light of the Work Statement provided in the Technical Proposal submitted in June, 1973 (Part 1, Appendix 3).

Initial investigations quickly indicated that tetrabutyl ammonium fluoroborate, the promising candidate identified in the original Technical proposal, provided insufficient conductivity, probably because of inadequate solubility, to give field grading at $60 \mathrm{~Hz}$. Therefore, quite early in the program, we screened a large number (approximately 35) of candidates. From these, we selected fifteen for evaluation in polyethylene. The experiments on these are covered in sections 5.2.1.2 and 5.2.1.3 of the original Technical proposal. In addition, we examined some of these additives in a vinyl acetate-ethylene-copolymer, since we expected to obtain higher conductivities, and optical observations were simplified.

since we soon realized that we needed to evaluate a relatively large number of additives, we decided to confine ourselves to one concentration ( $5 \%$ ) until one additive could be selected. We measured the DC resistivity and the frequency dependence of loss 
tangent at three temperatures and in most cases for two thicknesses. These results, reported in Appendix 2 and summarized in the text, cover section 5.2.1.2 in the Proposal.

Initial attempts to measure the field dependence of the loss tangent (Section 5.2.1.3) were unsuccessful because at the fields necessary to obtain a measureable effect, ( $1 \mathrm{MV} / \mathrm{cm}$ ), discharges in the measuring cell obviated the result. Later we were able to demonstrate this field dependence by measurements on a point to plane geometry. This was made possible by a mathematical analysis of the fields and conduction currents in the presence of field grading. Measurements, both in dielectric liquids ( $A C$ and $D C$ ) and polyethylene (DC), confirmed in a quantitative fashion the predicted field grading: Our analysis indicated also that the magnitude of the field grading effect should have produced a very large increase in tree inception voltage. Experimental results, however, show that this increase is marginal at $60 \mathrm{~Hz}$. In an attempt to check whether the absence of field grading in polyethylene at $60 \mathrm{~Hz}$ is due to a possible dispersion in the phenomenon we studied the effect of frequency on the conductivity in liquid solutions and on the tree inception voltage in polyethylene.

As predicted, the conductivity of polyethylene containing ionic salts was found to increase substantially on addition of a chlorinated biphenyl liquid (Pyranol 1499). However, although treeing was never observed in the sample, breakdown occurred at about the same voltage as in the absence of Pyranol. These results refer to section 5.2.1.5 in the Proposal.

Since we did not find a satisfactory additive known to be ionic, we did not consider it worthwhile to investigate solubilities as described in section 5.2.1.4. For the same reason we were unable to perform tasks described in sections 5.2.2 and 5.2.3.

In addition to the above, we tested two compounds which at first sight do not appear to be ionic, although the possibility cannot be ruled out that they operate through a field grading 
mechanism. One of these, ferrocene, completely inhibited treeing in our tests and in addition, raised the breakdown voltage substantially ( $100 \%$ ). Another, N, N'diphenyl.p-phenylene diamine, greatly increased the time to treeing in our long time tests although it did not improve the tree inception and breakdown voltage using our regular schedule. We are planning to investigate the mechanism by which these additives function in future work.

Before proceeding to an analysis for use in cables of the promising additives identified above, as proposed in section 5.2.3 of the original Technical proposal, it is suggested that the present work be continued in order to obtain additional data on field grading additives, on the mechanisms by which ferrocene. operates', and on the performance of ferrocene (or other mostpromising candidates) in cross-linked polytheylene. 


\section{FIELD CALCULATIONS}

\subsection{Introduction}

During our early consideration of the feasibility of using ionic additives to accomplish resistive grading, we examined a number of parameters and their influence on the phenomenon (Part 1: Section 1.5 and Appendices 2 and 3 ). No attempt was made at that time to develop a model which would predict the magnitude of the effect, that is the increase in treeing inception voltage to be realized with resistive grading. In this section we attempt to formulate such a model.

\subsection{Method}

In the interest of obtaining an analytical solution, we shall approximate the needle to plane geometry which we are using by concentric spheres as shown in Fig. l. For values of $r_{0} / R<1$ (in our case $r_{0}$ is $1.5 \mathrm{mils}$ and $R$ is $100 \mathrm{mils}$ ), it is believed that at least for semiquantitative purposes such an approximation is valid.

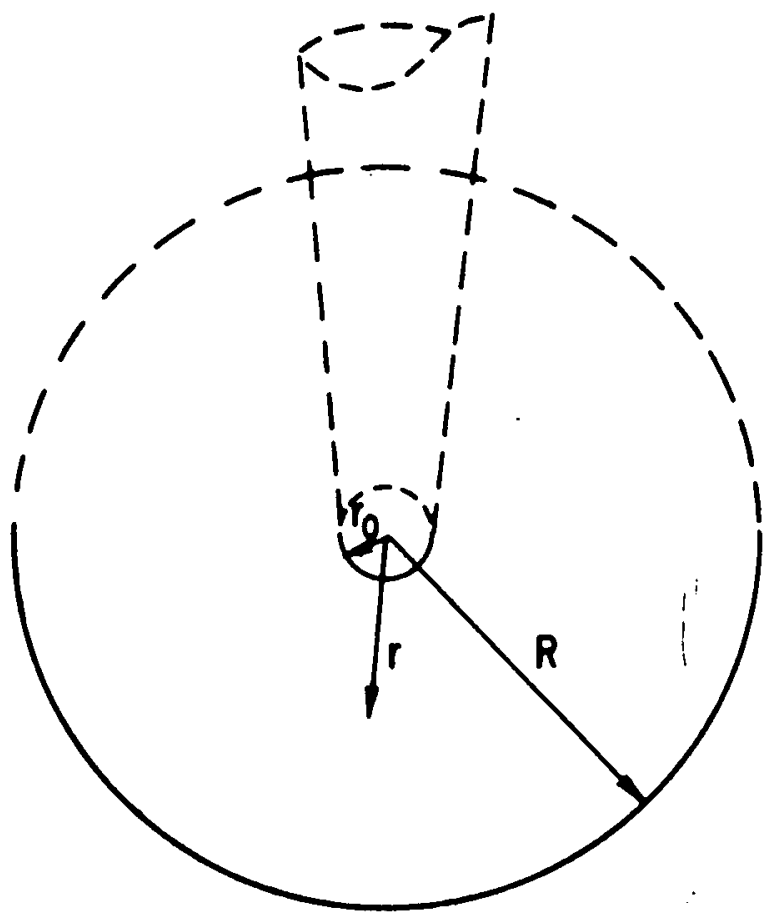

Fig. 1 Approximation of point-plane geometry by concentric spheres. 
The equations of interest are the following:

$$
\begin{aligned}
\nabla \cdot j & =0 \\
j & =\sigma E
\end{aligned}
$$

Here, $j$ is the real current density and $\sigma$ is the conductivity which in general will be a function of the field $E$. The first equation expresses the continuity of current, and the second is the equivalent of $\mathrm{ohm}$ 's law, but with a variable conductivity. We shall first solve these simultaneous equations without assuming an explicit functional form for $\sigma$, and then will introduce the onsager relationship assuming field enhanced ionic dissociation. The details of these calculations are given in Appendix 1.

\subsection{Results}

The general solution for the field is given in Appendix 1 by Eq. $(7-22)$ as

$$
E=\frac{4 v^{2}}{f^{2}\left[E\left(r_{0}\right)\right] \sigma r^{2}}
$$

where

$$
f\left[E\left(r_{0}\right)\right]=\int_{E\left(r_{0}\right)}^{0} \sigma^{-1 / 2} E^{-1 / 2} d E+\int_{\sigma\left(r_{0}\right)}^{\sigma} \sigma^{-3 / 2} E^{1 / 2} d \sigma(2-4)
$$

This solution assumes that $R>>r_{0}$, and is valid for any dependence of $\sigma$ upon $\mathrm{E}$.

If field-enhanced ionic dissociation occurs, then for low dielectric constant materials and for fields greater than about $1 \times 10^{5}$ volt $\mathrm{cm}^{-1}$, Onsager's treatment ${ }^{(1)}$ gives [cf. Eq. (7-14)] for the conductivity:

$$
\sigma=A \sigma_{0} E^{-3 / 8} e^{\alpha E^{1 / 2}}
$$


where

$$
A=0.17\left(\varepsilon T^{2}\right)^{3 / 8} \text { and } \alpha=\frac{4.39}{\left(\varepsilon T^{2}\right)^{1 / 2}}
$$

Here $\sigma_{0}$ is the zero-field conductivity, $\varepsilon$ is the dielectric constant of the medium, and $T$ is the absolute temperature. Using this form for $\sigma$, the function defined in Eq. (2-4) becomes [cf. Eq. $(7-28)]$.

$$
f\left[E\left(r_{o}\right)\right]=2 \mathrm{YA}^{-1 / 2} \sigma_{\mathrm{o}}^{-1 / 2}
$$

where $Y$ is expressed in terms of incomplete gamma functions (2) and is readily computed for any known value of the field at $r_{0}$. In Fig. 7-1 (Appendix 1 ), $Y$ is plotted against $E\left(r_{0}\right)$ for $a$ material with dielectric constant of 2.0 and for a temperature of $25^{\circ} \mathrm{C}$. The complete solution for the field, utilizing Eq. (2-6), is

$$
E(r)=\left[\frac{V^{2} \exp \left\{-\alpha[E(r)]^{1 / 2}\right\}}{Y^{2} r^{2}}\right]^{8 / 5}
$$

This is a transcendental equation and must be solved by iterative or graphical techniques.

We are primarily interested in the field at the point (i.e., at $r_{0}$ ) where treeing initiates. If we make the assumption that treeing begins when $E\left(r_{0}\right)$ just equals the intrinsic electric strength of the material, $E_{s}$, then Eq. (2-7) yields for the inception voltage for treeing

$$
V_{t}=E_{s} 5 / 16 Y_{0} \exp \left(\frac{\alpha E_{S}^{1 / 2}}{2}\right)
$$

Since in general the intrinsic strengths of materials of interest are greater than $10^{6}$ volt $\mathrm{cm}^{-1}$ (polyethylene is about $7 \times 10^{6}$ volt 
$\mathrm{cm}^{-1}$ ) (3), Fig. 7-1 indicates that $Y$ will be essentially a constant in this equation.

Equation (2-8) allows us to calculate the onset voltage for treeing in terms of the intrinsic strength and dielectric constant of the material and as a function of point radius and temperature, providing field-assisted ionic dissociation is occurring in accordance with the onsager theory. We have found that, with needles having radii of $3.81 \times 10^{-3} \mathrm{~cm}(1.5 \mathrm{mil})$, treeing initiates in polyethylene at $25 \mathrm{kV}$. Assuming that no grading occurs, then from Eq. $(7-25)$.

$$
v_{t}=r_{o} E_{s}
$$

and $\mathrm{E}_{\mathrm{s}}$ is calculated to be $6.6 \times 10^{6}$ volt $\mathrm{cm}^{-1}$. This corresponds closely to the intrinsic strength, $E_{S^{\prime}}$ for polyethylene. Utilizing this value in Eq. (2-8) we calculate for the expected treeing orset voltage with grading $8.0 \times 10^{8}$ volt. Of course, breakdown would be expected at much lower voltages than this owing to thermal runaway (4) but the result shows the very large effect to be expected from this type of field grading.

Another approach which tends to minimize errors due to the approximation of a point to plane geometry by concentric spheres is to examine the ratio of voltages required to reach $E\left(r_{0}\right)=E_{s}$ with and without grading. Utilizing Eqs. (2-8) and (2-9) this ratio is

$$
\gamma=E_{S}^{-11 / 16} Y \exp \left(\frac{\alpha E_{S}^{1 / 2}}{2}\right)
$$

It is interesting that this ratio is independent of $r_{0}$. Values of $\gamma$ are plotted in Fig. 2 against the electric strength of the material. As was noted above, the ratio is very large and, as might be expected, increases dramatically with increasing $\mathrm{E}_{\mathbf{S}}$. This points up an important result, namely that additives used to promote grading must not seriously degrade the intrinsic strength of the material. 


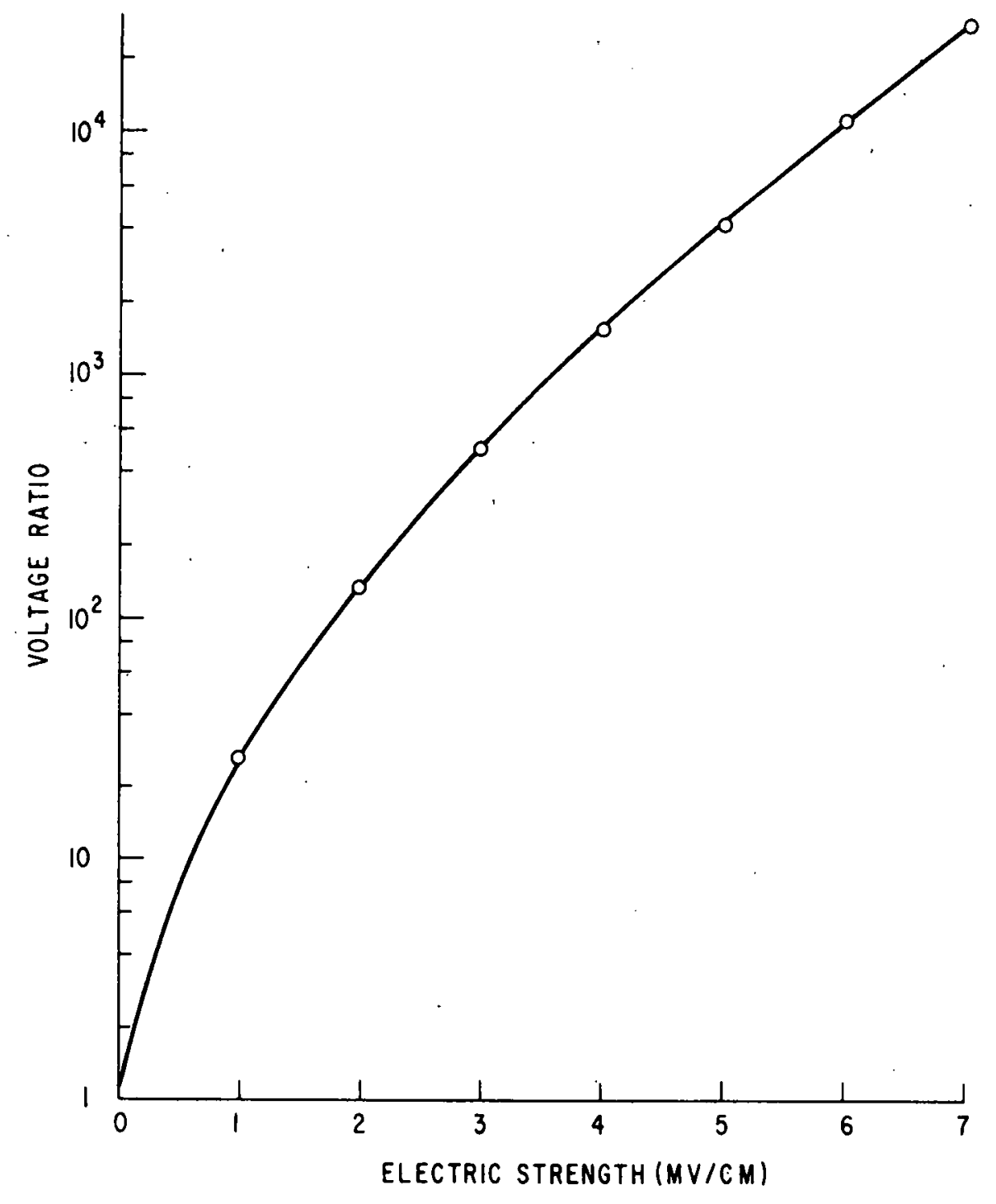

Fig. 2 Ratio of calculated treeing inception voltage with and without grading as a function of electric strength of the material.

The analysis conducted above may profitably be applied to the propagation step of growing trees. If we assume that discharges in the tree channels transfer the voltage on the point to the end of the channels, and that the requirement for propagation again is that the field in the solid exceeds $E_{S}$, then we note from Eq. $(2-8)$ that the voltage required is strongly dependent on $r_{0}$, the radius of the channel. In general, since the radius of the channels is much smaller than that of the points, the 
propagation voltage is much smaller than that calculated above for initiation, providing that the voltage drop in the channel is not too large. As far as can be observed, even from their inception, tree radii $(\sim l \mu)$ are much smaller than those of our points so that it might be argued that initiation actually occurs on a small electrical stress riser on the point. Because of this, it cannot be concluded with certainty that field grading does not occur simply because the initiation voltages observed are much lower than those predicted by Eq. (2-8). It is interesting to calculate, using Eq. (2-8), the values of $r_{0}$ and voltages required to reach $E_{s}$ for various values of $E_{s}$. Such plots are shown in Fig. 3. These results again show the very important influence of

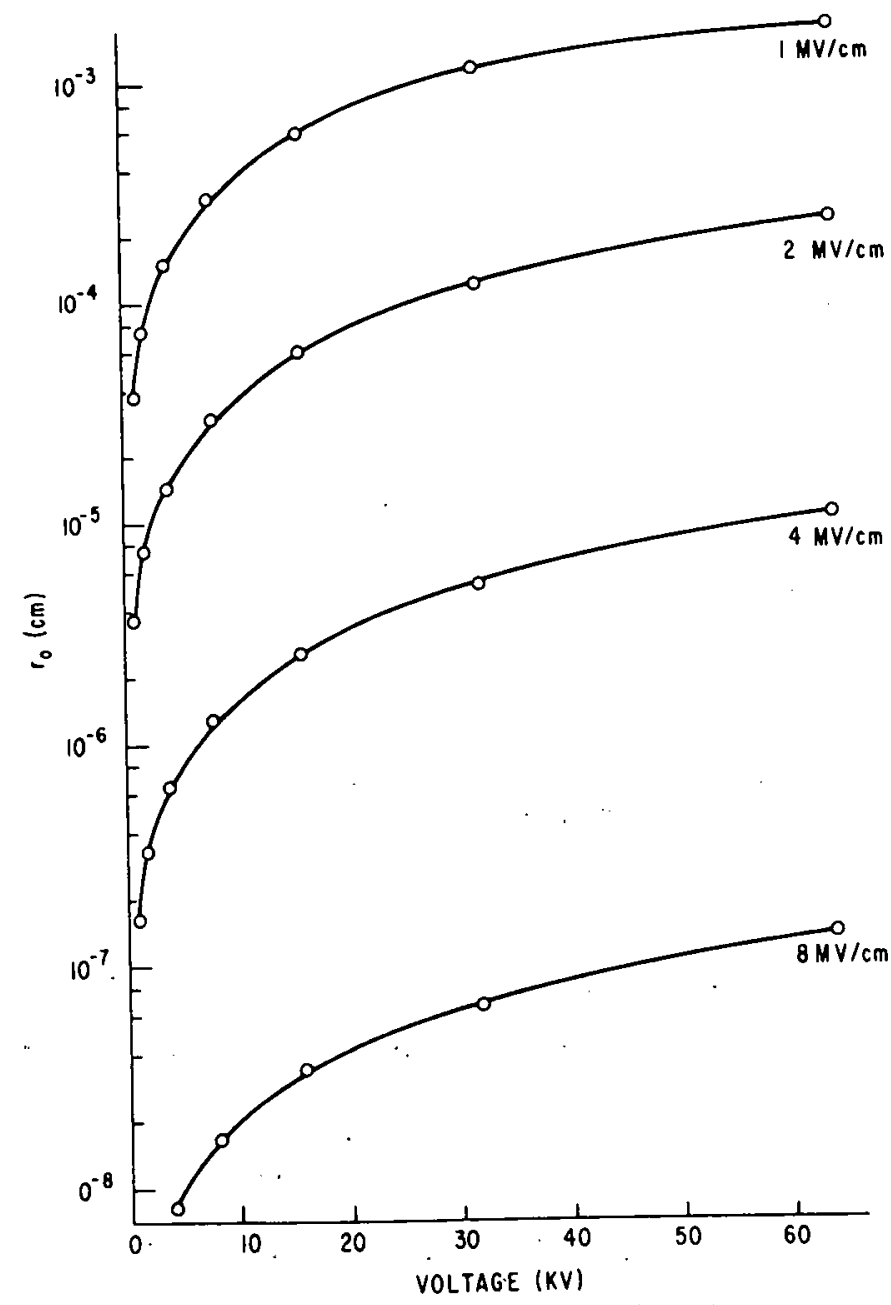

Fig. 3 Dependence of calculated treeing inception voltage on point or channel radius, ro, with materials of various intrinsic electric strengths 
intrinsic material strength on the possibility of tree growth if grading is occurring. Thus, for $E_{S}=8 \mathrm{MV} \mathrm{cm}-1$, trees of radius not much greater than $10 \AA$ would be possible. On the other hand, with voltages of only $25 \mathrm{kV}, 1_{\mu}$ channels are possible with ma-

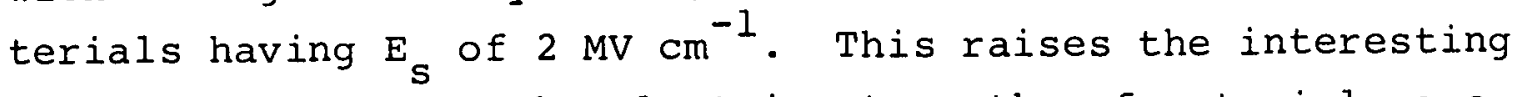
question of whether the electric strengths of materials are lowered appreciably at the ends of the propagating channels by the impinging discharges in the channels. Of course, a complete theory of tree propagation .long the lines discussed above would require a knowledge of the voltage gradient in the discharging channels as a function of their radii.

To summarize the points described in this section:

1. We have approximated a point to plane geometry by concentric spheres.

2. We have solved analytically the field equations for materials showing field-dependent conductivities obeying the onsager theory.

3. The results indicate that the field-grading effect, if it is occurring at our $60 \mathrm{~Hz}$ voltages, should be very large.

4. The magnitude of the field grading effect increases markedly with increase in the material electric strength. This suggests that effective additives should not markedly degrade this property.

5. Our model is extended to tree propagation and has led to a relation between tree size $\left(r_{0}\right)$, voltage- and material electric strength. A complete theory along these lines requires a knowledge of such parameters as the voltage gradient in discharging channels and $E_{s}$ of the material on which discharges are impinging. 
III. CURRENT MEASUREMENTS IN POINT-PLANE GEOMETRIES

\subsection{Introduction}

The results of the field calculation in section II suggested that it might be possible, from measurements of currents from points as a function of voltage, to observe departures from ohmic behavior which would be indicative of enhanced field dissociation and thus of field grading. In this section we shall describe the results of such measurements.

\subsection{Method}

Referring to Appendix 1, Eqs. (7-2) and (7-10) may be combined to give the current density to be expected in a concentric sphere geometry

$$
j=\frac{B}{r^{2}}
$$

Substituting for B from (7-20) gives

$$
j=\frac{4 V^{2}}{r^{2} f^{2}\left[E\left(r_{0}\right)\right]}
$$

where $f\left[E\left(r_{0}\right)\right]$ is the same as that described in section II, given by Eq. (2-4)

For Ohmic materials, Eq. (2-4) or (7-23) gives

$$
f\left[E\left(r_{0}\right)\right]=\frac{\left.-2\left[E\left(r_{0}\right)\right]\right]^{1 / 2}}{\sigma_{0}^{1 / 2}}
$$

so that Eq. $(3-2)$ becomes

$$
j=\frac{\operatorname{vor} 0}{r^{2}}
$$

when $E\left(r_{0}\right)$ is substituted from (7-25). For materials with conductivities obeying the onsager relation, $f\left[E\left(r_{0}\right)\right]$ is given by Eq. $(2-6)$ and 


$$
j=\frac{A \sigma_{0} V^{2}}{r^{2} Y^{2}}
$$

It, therefore, seemed feasible to show whether field-enhanced dissociation occurred from a determination of the form of the dependence of current on voltage.

For our initial measurements we chose to examine hydrocarbon liquids containing ionizable salts, since relatively large conductivities could be obtained and time constants shorter than the period of a $60 \mathrm{~Hz}$ wave were readily attainable. A 1.5 mil radius needle was inserted in a solution of hexadecane containing $0.144 \%$ $\mathrm{AD} 48 *$ in a guarded Pyrex cell such as shown in Fig. 4. Our first measurements were made using a DC voltage (variable from 0-5 kV)

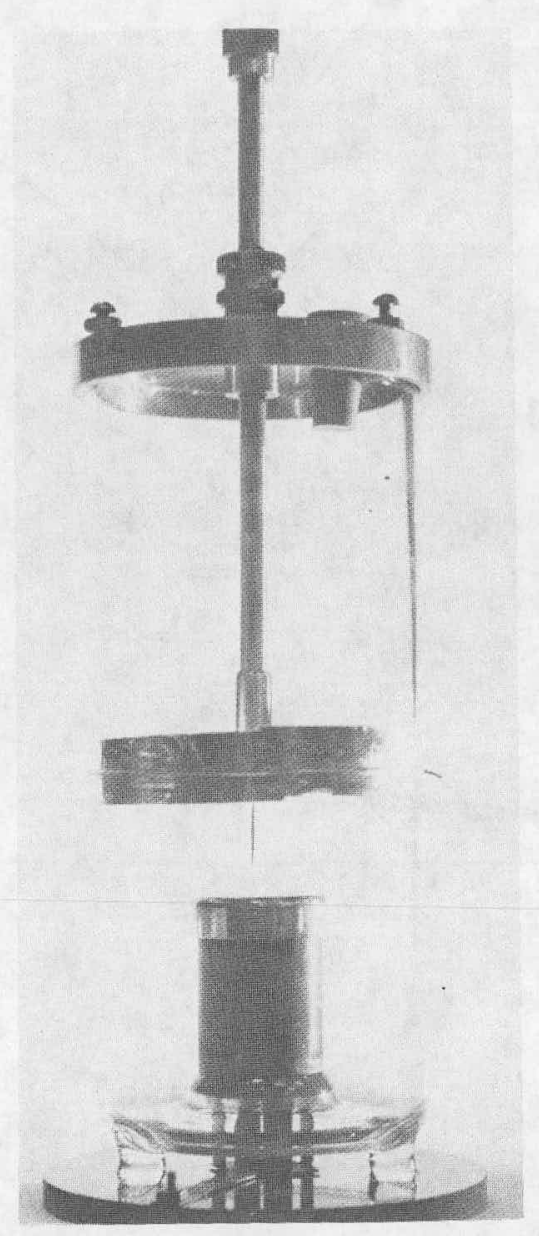

Fig. 4 Cell for measuring conduction currents for needle to plane geometries in liquids.

${ }^{*} \mathrm{Cf}$. Sec. 4.1 .1 
and measurements of current were made with a Model $600 \mathrm{~A}$ Keithley electrometer. In Fig. 5 we have plotted I-V curves for two depths of penetration of our standard needle into the liquid. As might be expected, the current increases with increasing depth of penetration since the number of parallel current paths increases. In both cases there is a departure from linearity in the direction to be expected from Eq. (3-5).

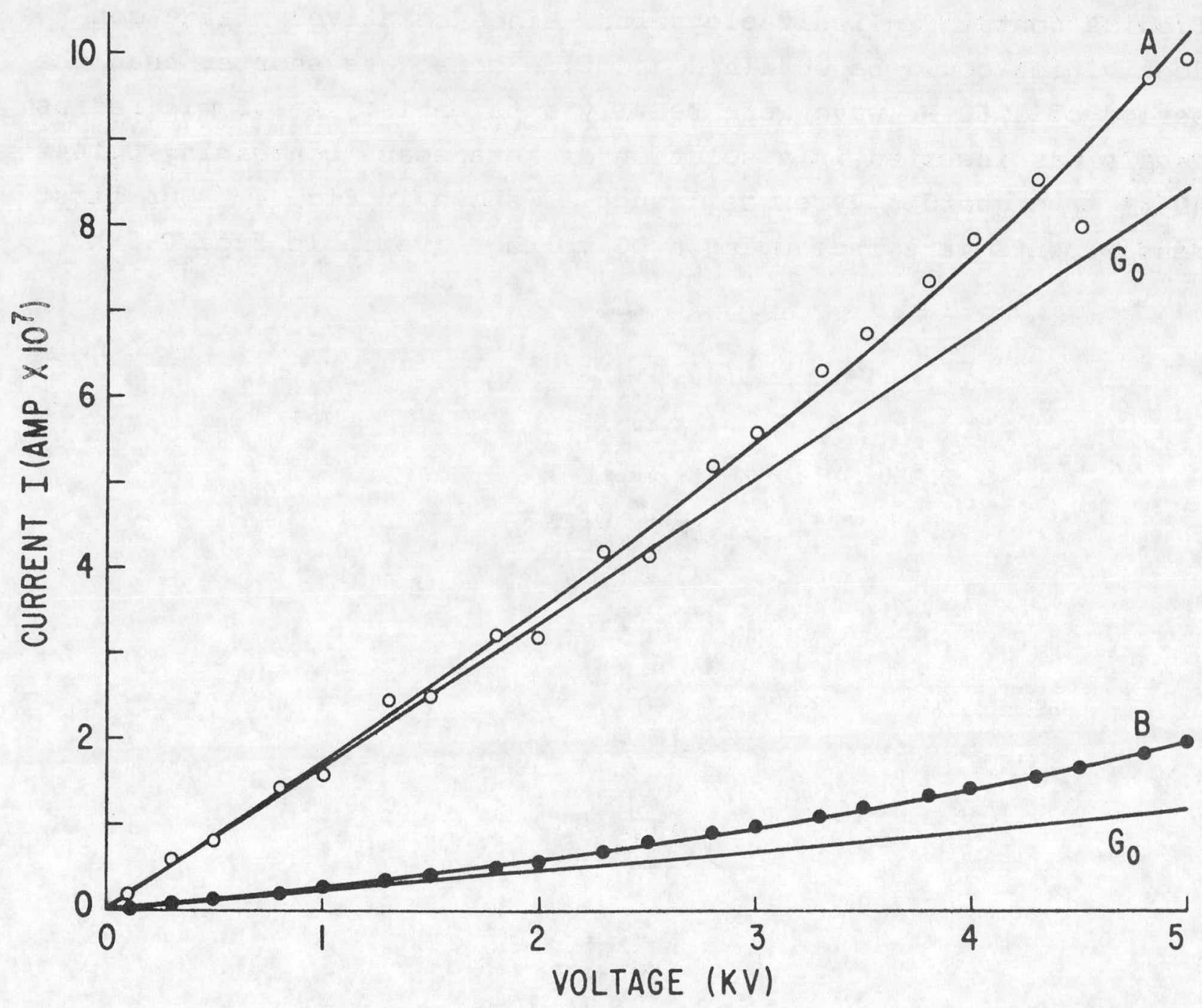

Fig. 5 Current-voltage characteristics for needle-plane in hexadecane containing $0.144 \%$ AD48. Spacing $6 \mathrm{~mm}$. Needle point depth in liquid: $A \sim 2 \mathrm{~cm}, \mathrm{~B} \sim 0.2 \mathrm{~cm}, \mathrm{G}_{0}$ refers to conductance expected for Ohmic behavior. 
Fig. 6 indicates the model circuit we use, as a first approximation, to represent our system. We assume that the hemispherical tip of the needle represents the high field region, so that field-enhanced dissociation occurs here to give a conductance varying with voltage, $G(V)$, and that other parallel paths are represented by a voltage-independent conductance $G^{\prime} \circ$. With this simplified model and using Eq. (3-5), the total current $I$ is given by

$$
I=2 \pi r_{0}^{2} j+G_{0} V=\frac{2 \pi A \sigma o V^{2}}{Y^{2}}+G_{O} V
$$

where $G_{\circ}$ includes $G_{\circ}^{\prime}$ and the low field conductance paths from the hemispherical tip. Thus, a plot of $I-G_{0} V$ against $V^{2}$ should give a straight line, if $\mathrm{Y}$ is constant and providing the conductivity varies with field according to the onsager relation. Go is measured with a low voltage DC bridge. In all measurements on liquids, the needle point was $20.1 \mathrm{~cm}$ beneath the liquid surface and the point to plane spacing was $0.6 \mathrm{~cm}$.

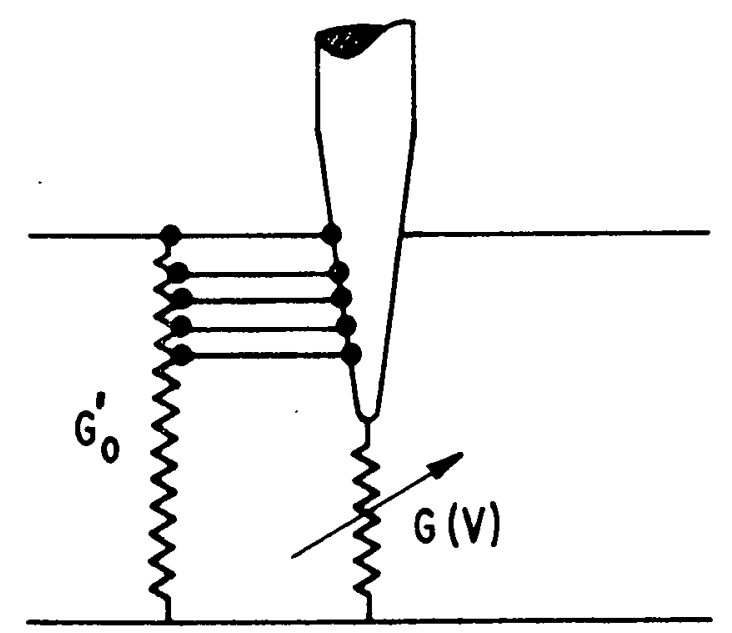

Fig. 6 Model circuit showing voltage-independent conductance of parallel paths, $G_{O}^{\prime}$, and voltage-dependent conductance from point, $G(V)$.

\section{3 Results}

Results for solutions of tetrabutyl ammonium fluoroborate (TBAFB) in benzene ranging in concentration from $0.025 \%$ to $0.1 \%$ 
by weight are shown in Figs. 7 and 8. In Fig. 7. current is plotted against voltage for the highest and lowest concentrations. Included are the currents to be expected based on low field measurements, labeled $G_{O}$. The upward departures of the experimental data from the linear behavior is clearly shown indicating an increase in conductance with voltage. In Fig. 8 we have plotted $I-G_{O} V$ for all four concentrations against $V{ }^{2}$. With the exception of a slight upward trend at the highest concentrations and fields, a fairly good proportionality is found.

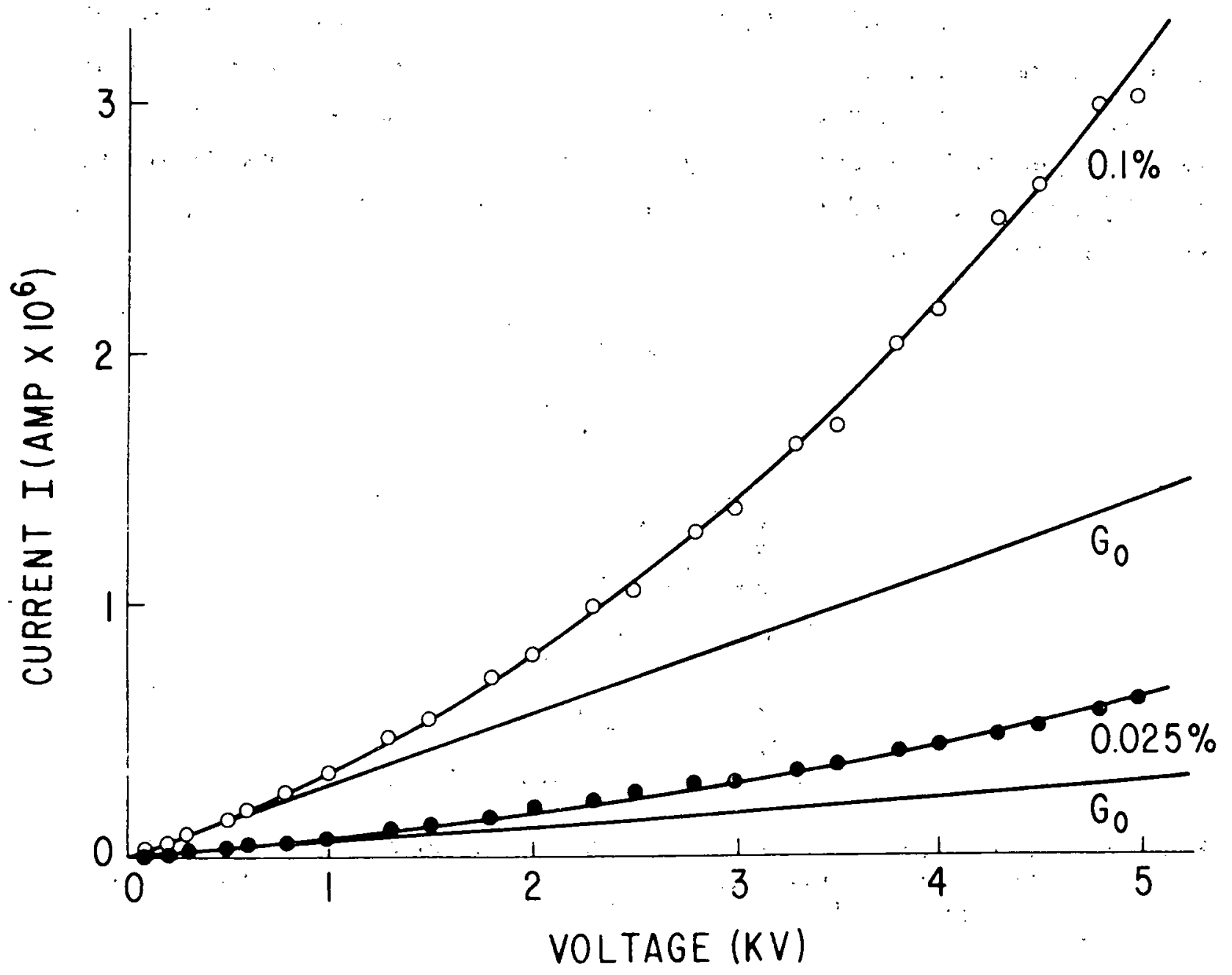

Fig. 7 Point-plane current-voltage characteristics for $0.1 \%$ and $0.025 \%$ TBAFB in benzene. $\mathrm{G}_{\mathrm{O}}$-low field conductances, DC. 


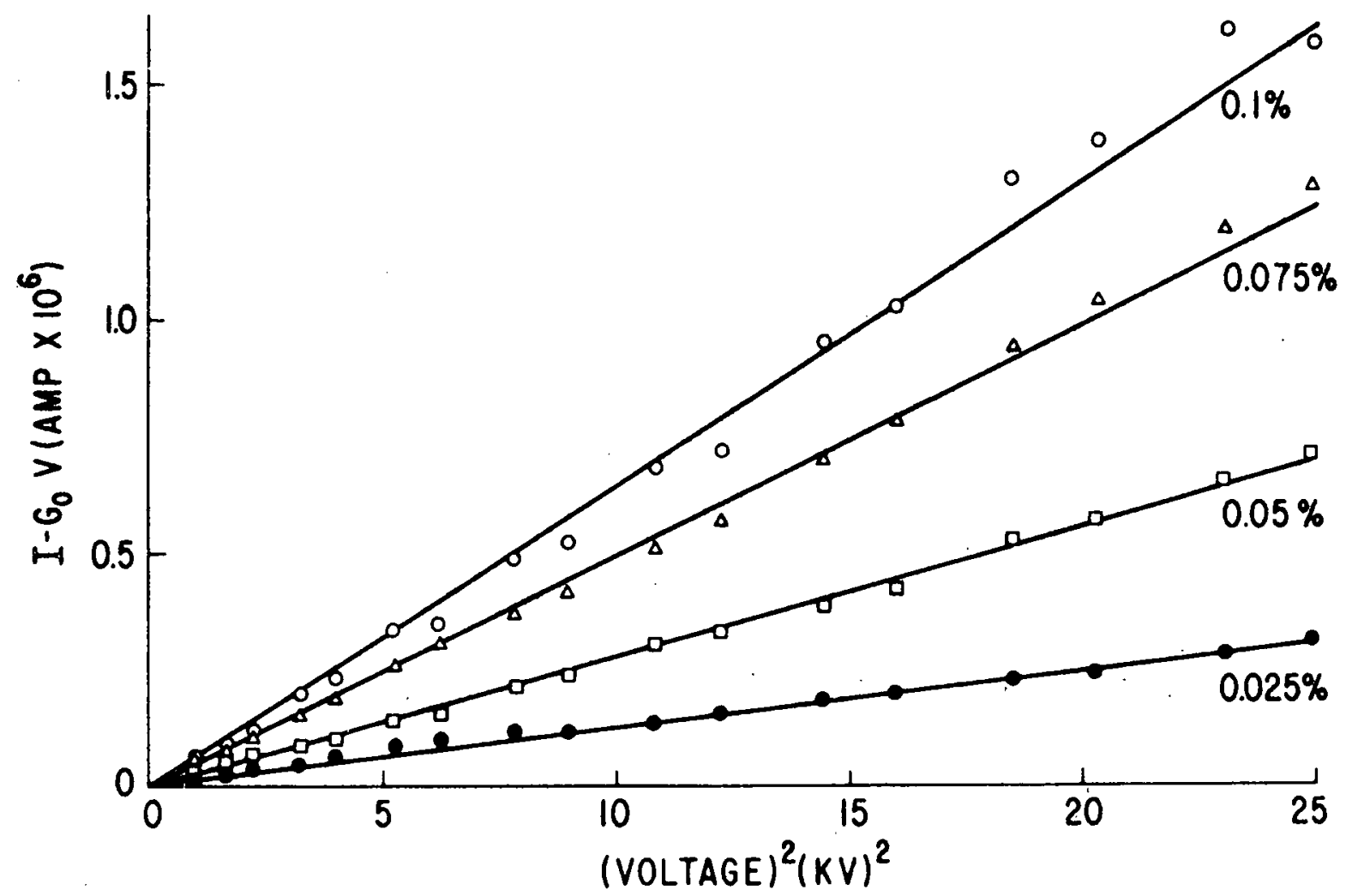

Fig. 8 Relation between nonlinear voltage-dependent portion of current and the square of voltage for four concentrations of TBAFB in benzene, DC.

Similar results were found for solutions of $0.02 \%$ to $0.64 \%$ AD48 in hexadecane, a sixteen carbon atom aliphatic hydrocarbon whose structure is similar to that of polyethylene. Fig. 9 shows current voltage plots for two concentrations and again marked deviations from the linear behavior expected for ohmic systems are noted. Plots of $I-G_{O} V$ against $V^{2}$ for all six concentrations are given in Figs. 10 and 11 . Again good linearity is observed.

It was of course, of considerable interest to make similar measurements with $60 \mathrm{~Hz}$ voltages, to ascertain whether similar evidence for field-enhanced dissociation could be seen under AC conditions. Initial data showed an essentially linear dependence of I on $V$. When it was realized that even with the high conductivities of these solutions the current was overwhelmingly capacitive, we made further measurements of $60 \mathrm{~Hz}$ conductances using a Schering bridge. In this case, since $I=G V$ (we refer to the real current only), Eq. (3-6) predicts a linear relation between 


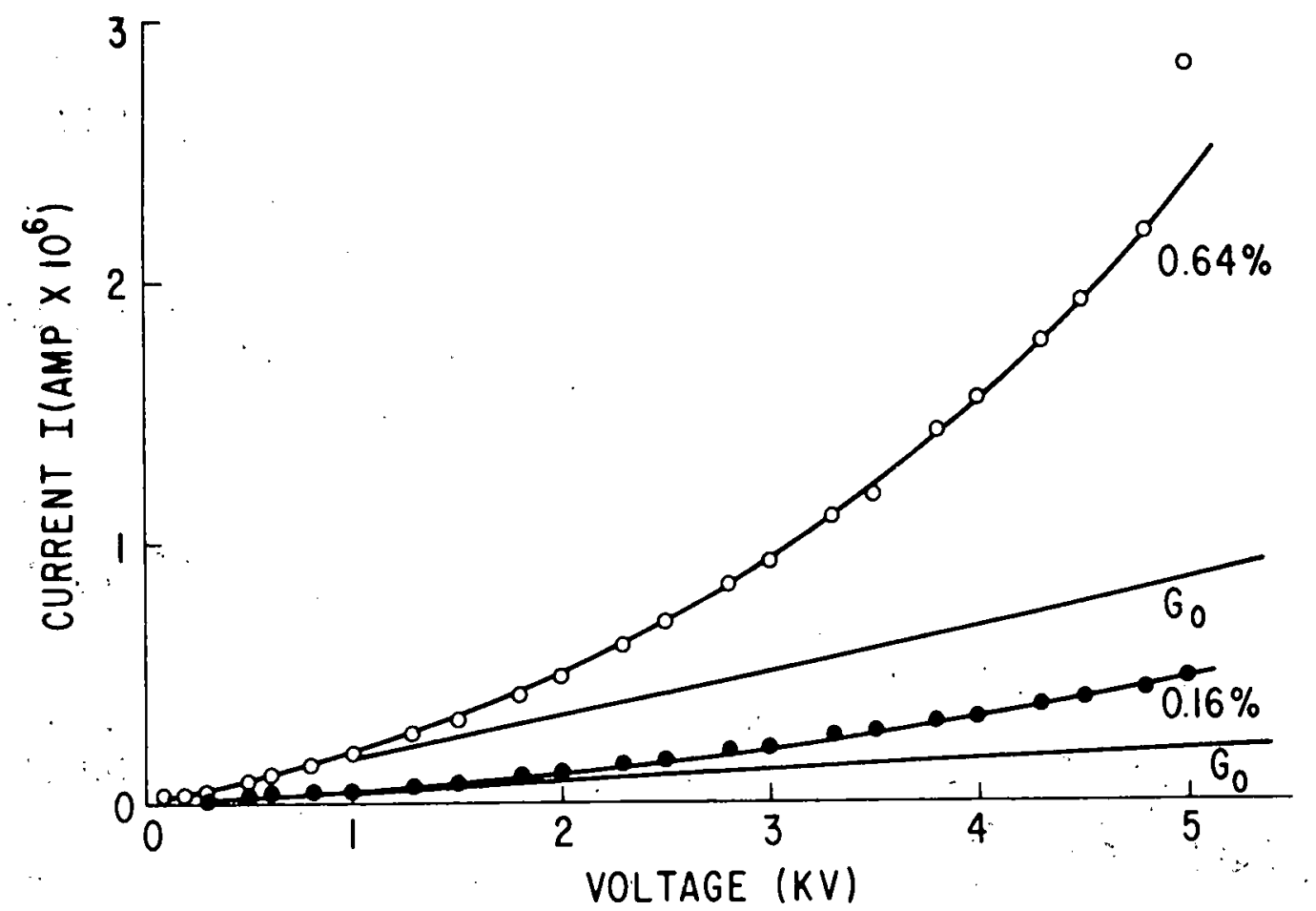

Fig. 9 Point-plane current-voltage characteristics for $0.16 \%$ and $0.64 \% \mathrm{AD} 48$ in hexadecane. $\mathrm{G}_{\mathrm{O}^{-}}$ low field conductances, DC.

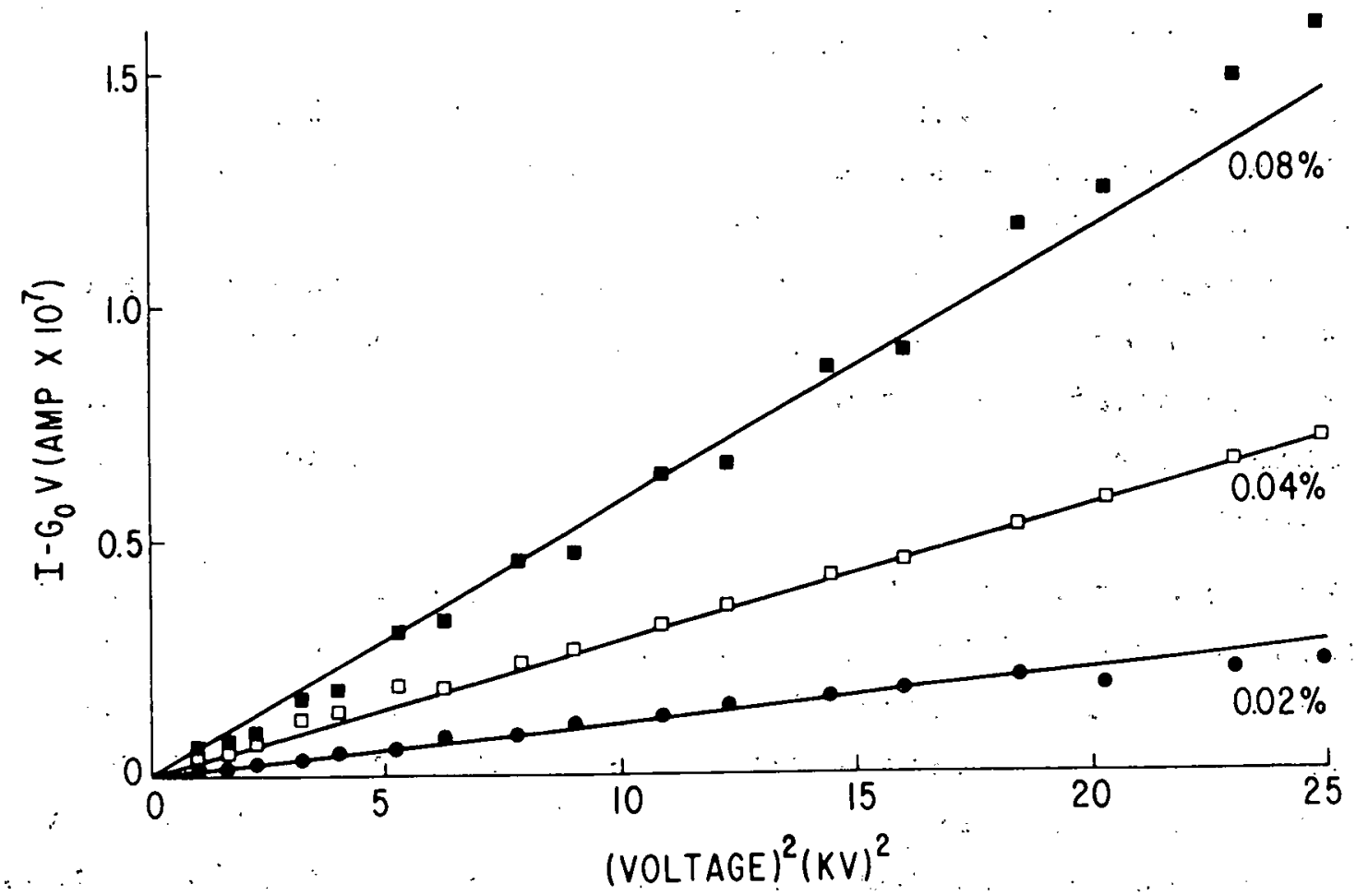

Fig. 10 Relation between nonlinear voltage-dependent portion of current and the square of voltage for three concentrations of $\mathrm{AD} 48$ in hexadecane, $\mathrm{DC}$. 


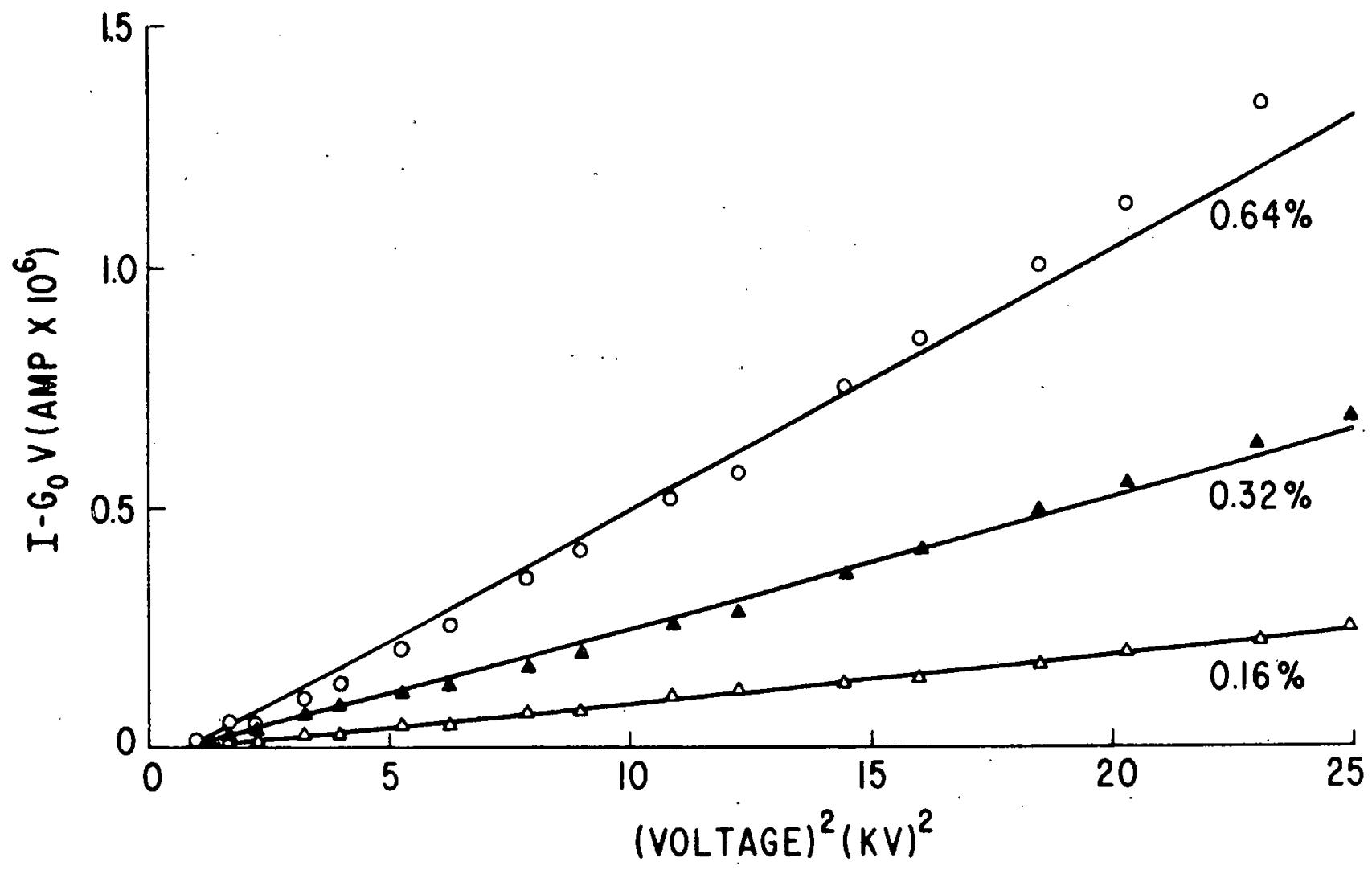

Fig. Il Relation between nonlinear voltage-dependent portion of current and the square of voltage for three concentrations of $A D 48$ in hexadecane, $D C$.

$G$ and $V$ with a positive intercept on the $G$ axis. As shown in Figs. 12 and 13, these expectations are largely substantiated for both the benzene-TBAFB and the hexadecane-AD48 systems. As with DC, there is evidence for some departure at the highest concentrations of added salts. In this connection, it should be noted that the onsager theory assumes the salt to be present as only free ions and neutral ion pairs. It is well known (.5) that, at higher concentrations, ion triplets and even higher multiplets will form in low dielectric constant media causing departures in conductivity predicted by theories involving only one equilibrium.

Equation (3-6) predicts that, the slopes of the lines in Figs. 8, 10, 11, 12 and 13 should be proportional to the zero (low) 


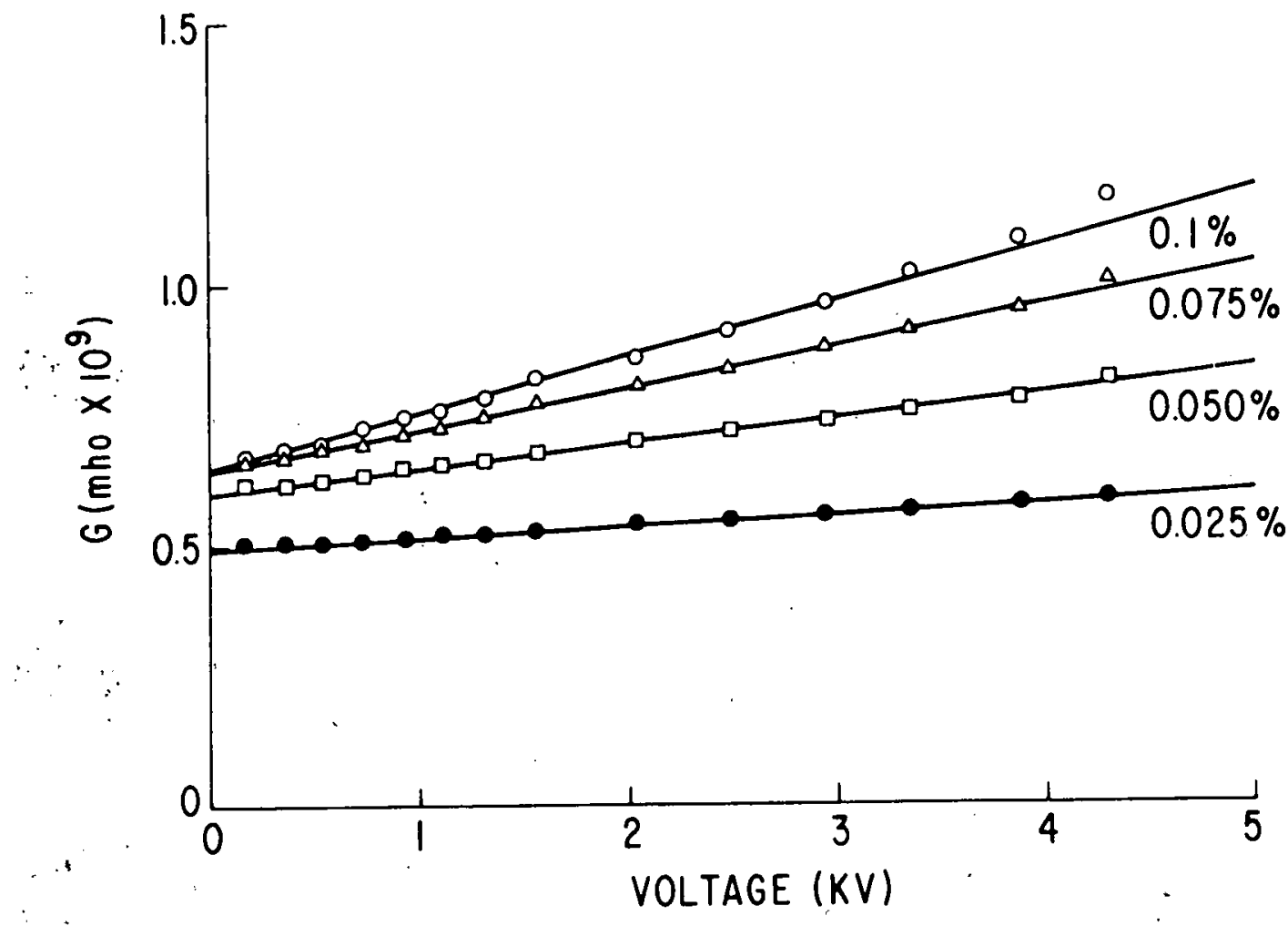

Fig. 12 Dependence of $A C$ conductance on voltage for several concentrations of TBAFB in benzene, $60 \mathrm{~Hz}$.

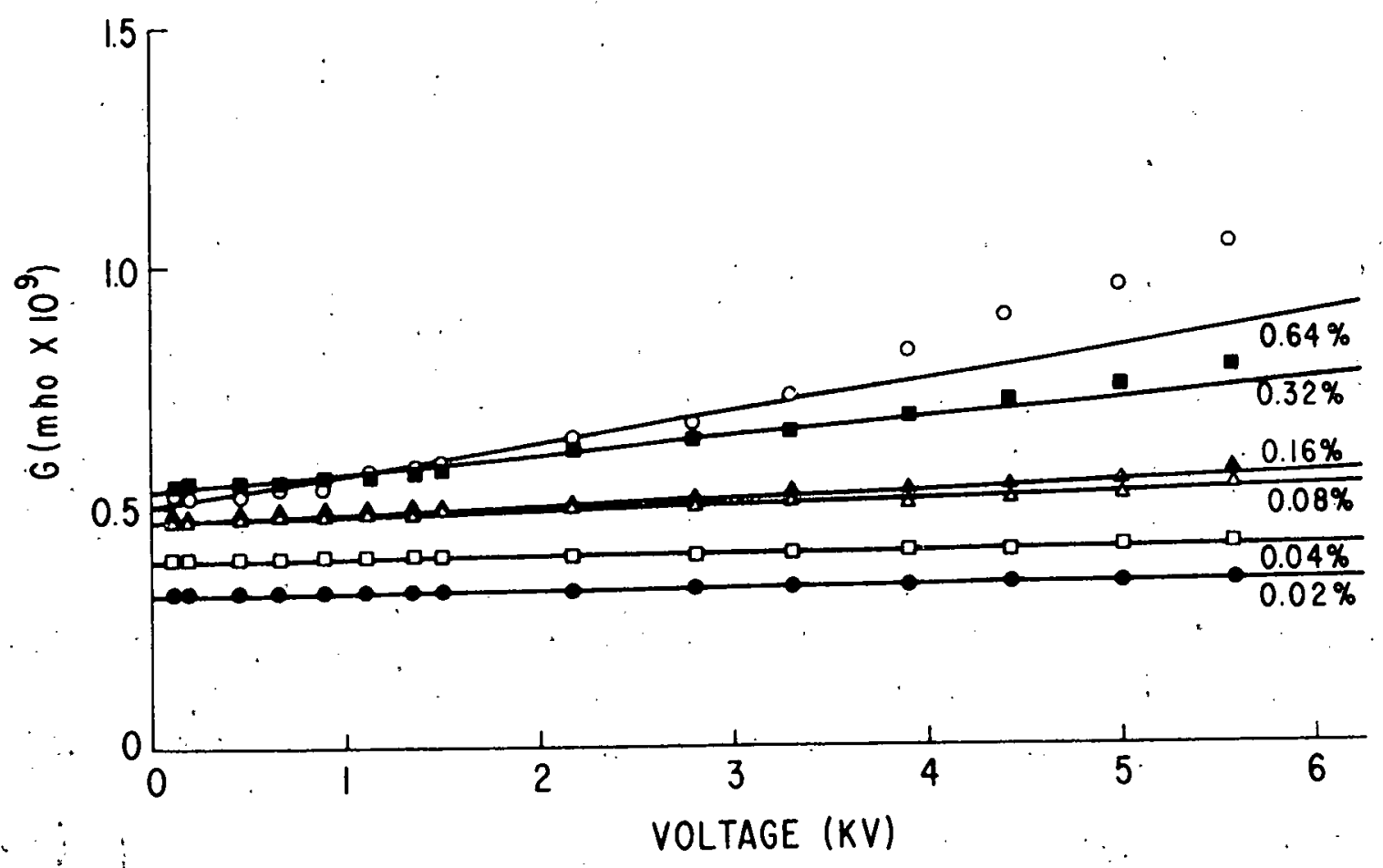

Fig. 13 Dépendence of $A C$ conductance on voltage for several concentrations of $\mathrm{AD} 48$ in hexadecane; $60 \mathrm{~Hz}$. 
field conductivity of the solutions. That this is so is seen in Fig. 14 for the DC results and in Fig. 15 for the AC results, for our two solutions.

The above results encouraged us to look for similar departures from ohmic behavior in polymers containing ionic additives, even though the conductivities were orders of magnitude lower.

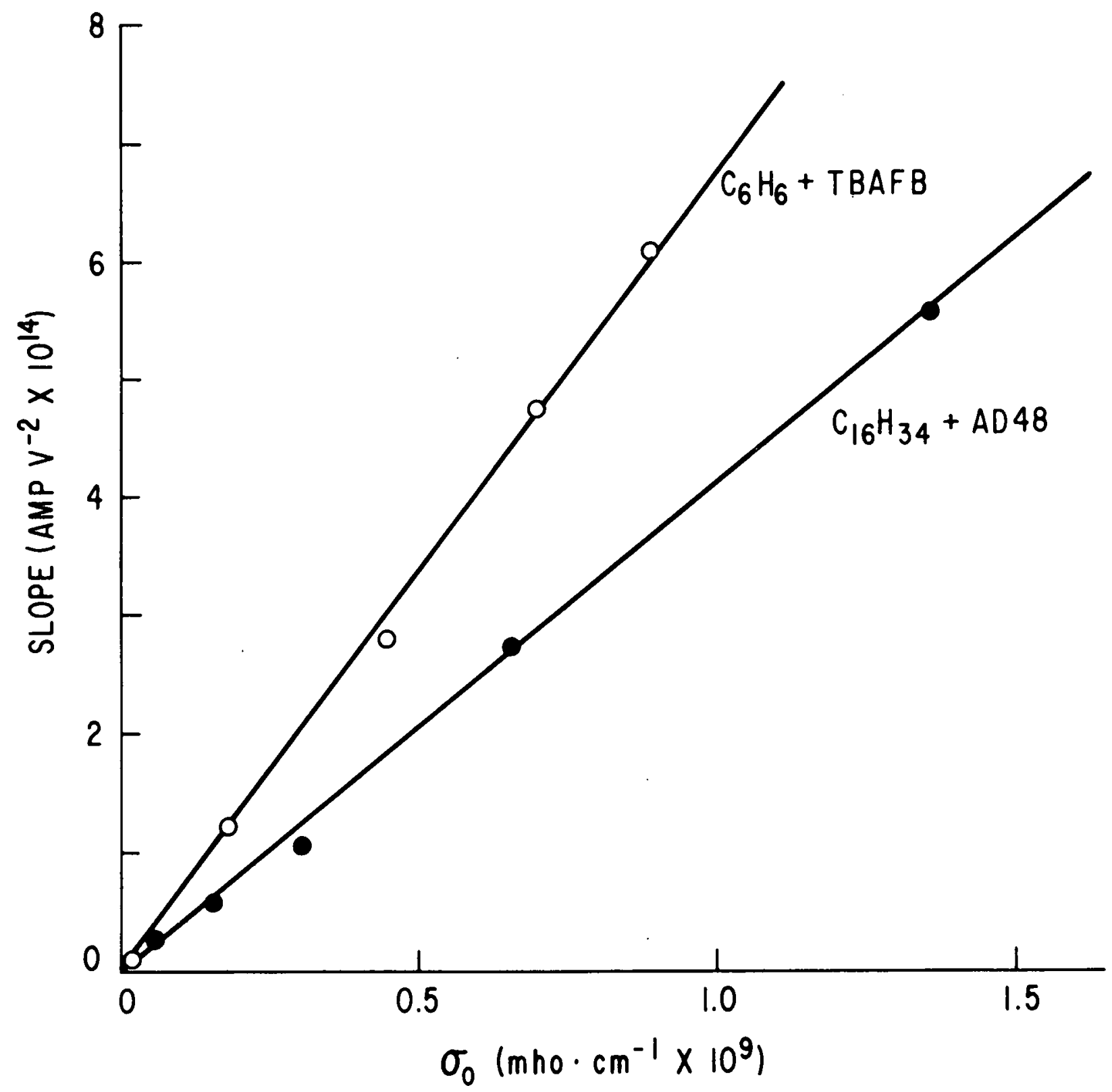

Fig. 14 Dependence of experimental slope of Eq. (3-6) on solution conductivity, DC. 


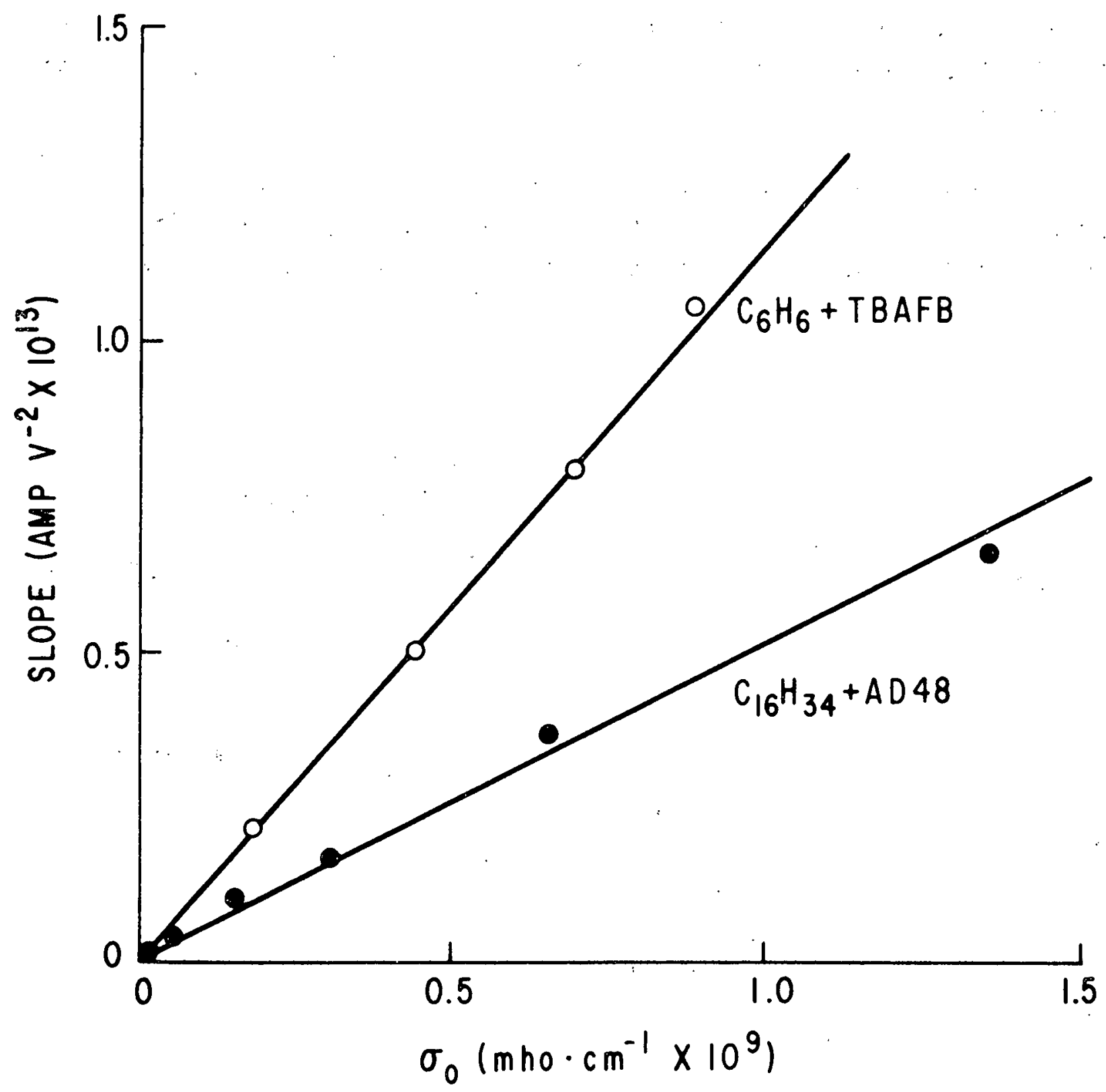

Fig. 15 Dependence of experimental slope of Eq. (3-6) on solution conductivity, $60 \mathrm{~Hz}$.

We chose first one of our most conducting polymeric systems, a polyvinyl acetate - polyethylene copolymer (PVAP) containing 21\% pentachlorodiphenyl (Pyranol) and 5\% sodium oleate by weight. The sample was disk-shaped with a diameter of approximately l" and thickness of 0.125". A 1/2" diameter silver electrode was painted on one side and a standard $3 \mathrm{mil}$ diameter steel needle was inserted in the other face to a.depth of approximately $0.025 "$. 
A silver guard was painted on the edge of the disk. A DC currentvoltage plot is shown in Fig. 16, where departures from linearity similar to those observed in liquids are noted. A plot of $I-G_{0} V$ against the square of the voltage is shown in Fig. 17, and very nearly linear behavior is observed.

Finally, in an effort to substantiate the field grading phenomenon in polyethylene, we examined a mixture containing 5\% of sodium lauryl sulfate in polyethylene showing one of the highest low field conductivities we have observed with this

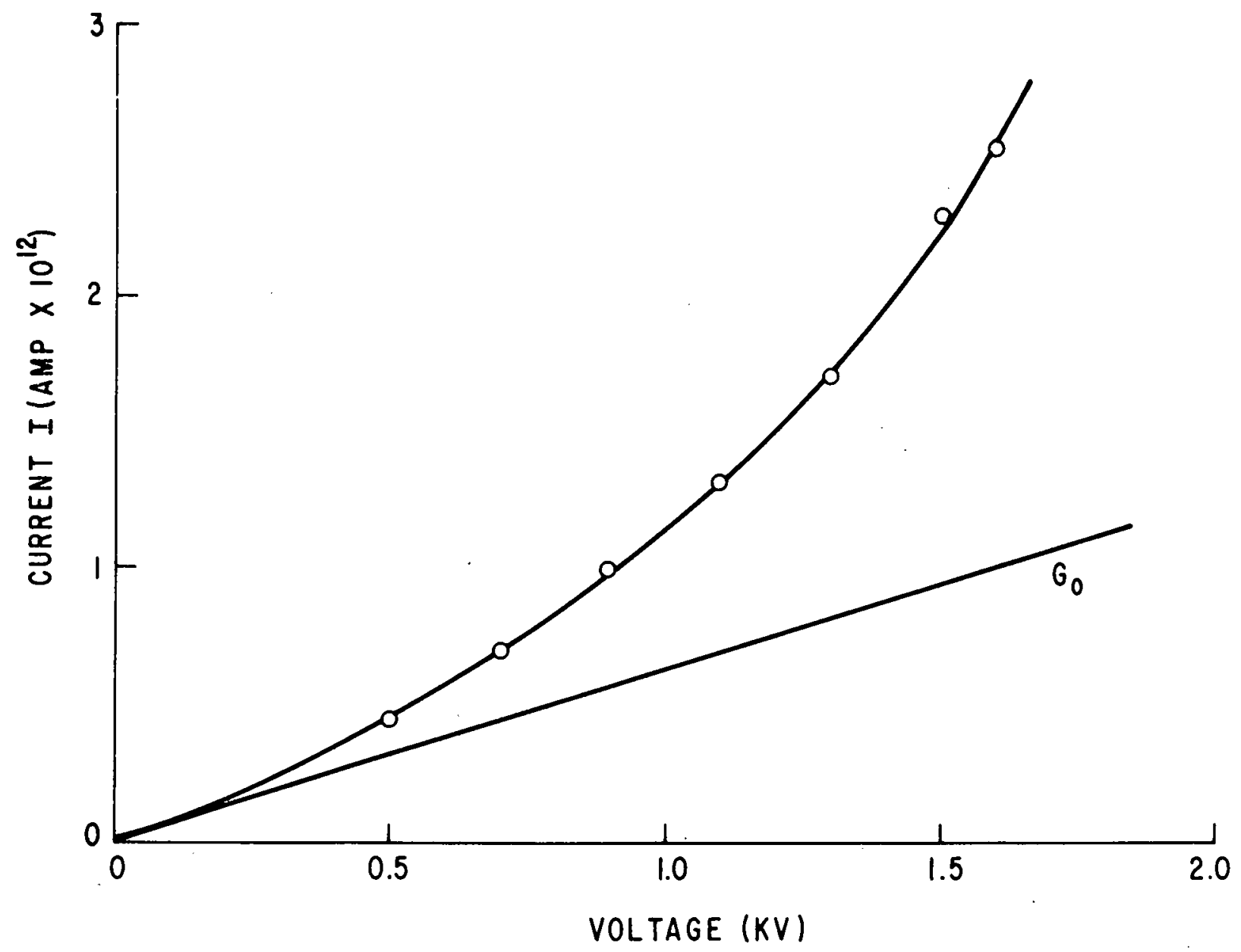

Fig. 16 Point-plane current-voltage characteristics for a polyvinyl acetate-polyethylene copolymer containing $21 \%$ pentachlorodiphenyl and 5\% sodium oleate by weight. $\mathrm{G}_{\mathrm{O}}$-low field conductance, DC. 


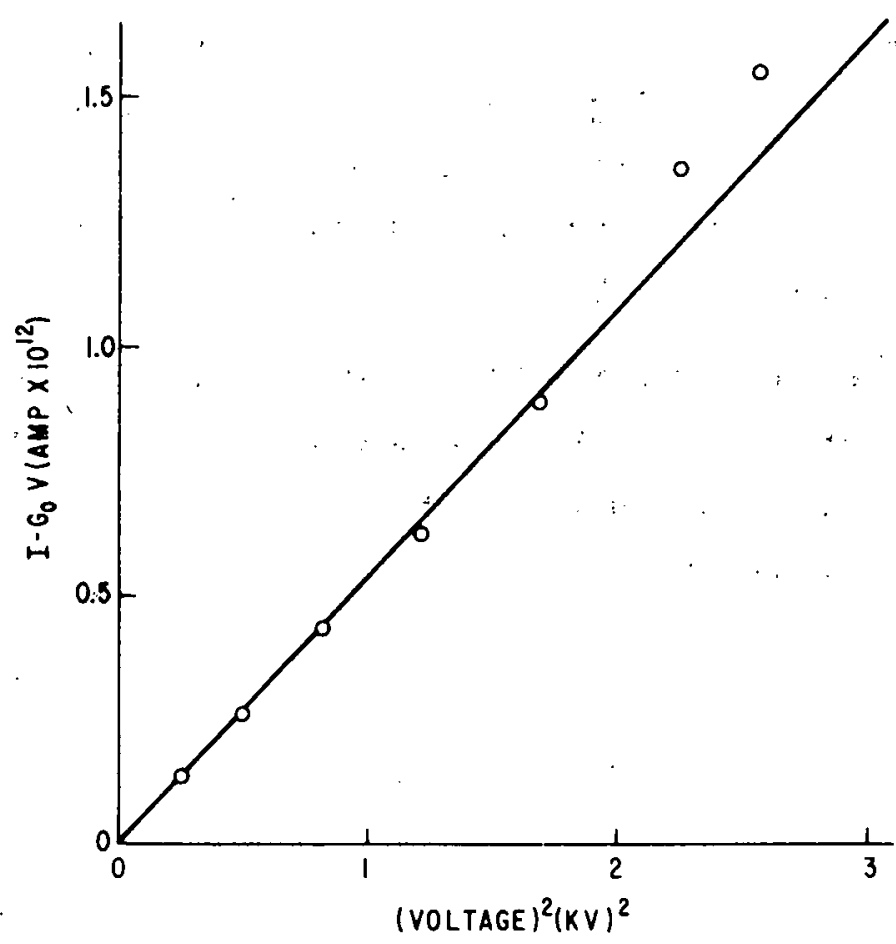

Fig. 17 Relation between nonlinear voltage-dependent portion of current and the square of voltage for a polyvinyl acetate-polyethylene copolymer containing 21\% pentachlorodiphenyl and $5 \%$ sodium oleate by weight, DC.

polymer. The DC current-voltage curve is shown in Fig. 18, and the departure from linearity to be expected from field enhanced dissociation is seen. Plots, corrected for low-field conduction, are again found to be proportional to the square of the voltage, as seen in Fig. 19.

$60 \mathrm{~Hz}$ Schering bridge"measurements on both of the polymeric systems described above failed to show a conductance which increased with voltage, as was observed with the salt-containing liquids, also described above. This is not surprising when we calculate the magnitudes of the effect. Thus; we expect; utilizing Eq. (3-6) the increase in G:

$$
\begin{aligned}
\Delta \mathrm{G}=\frac{2 \pi \mathrm{A} \sigma_{\mathrm{O}} \mathrm{V}}{\mathrm{Y}^{2}} & =\frac{2 \pi \times 16.3 \times \sigma_{\mathrm{O}} \mathrm{V}}{2500^{2}} \\
& =1.6 \times 10^{-5} \sigma_{\mathrm{O}} \mathrm{V}
\end{aligned}
$$




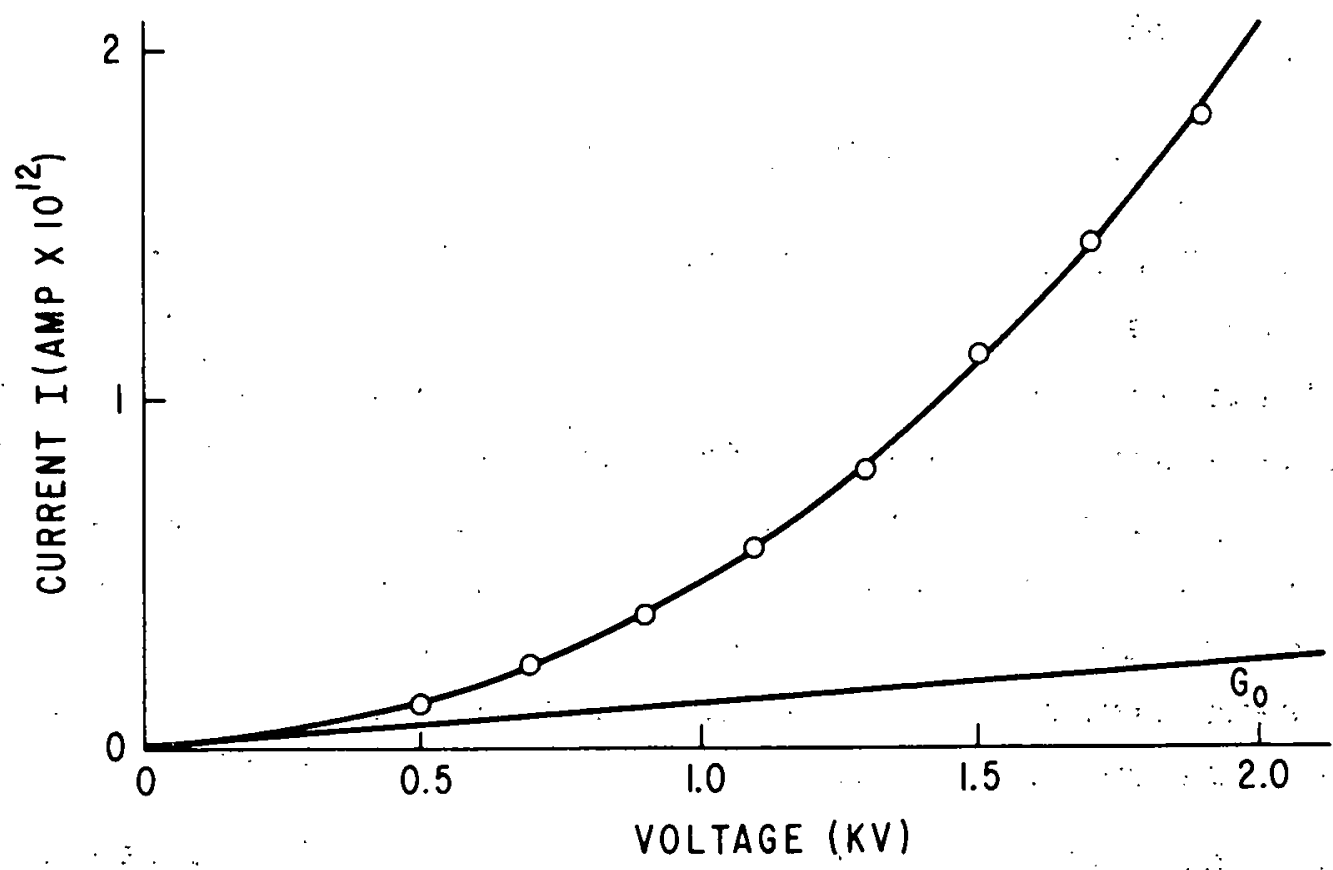

Fig. 18 Point-plane current-voltage characteristics for polyethylene containing 5\% sodium lauryl sulfate. Go-low field conductance, DC.

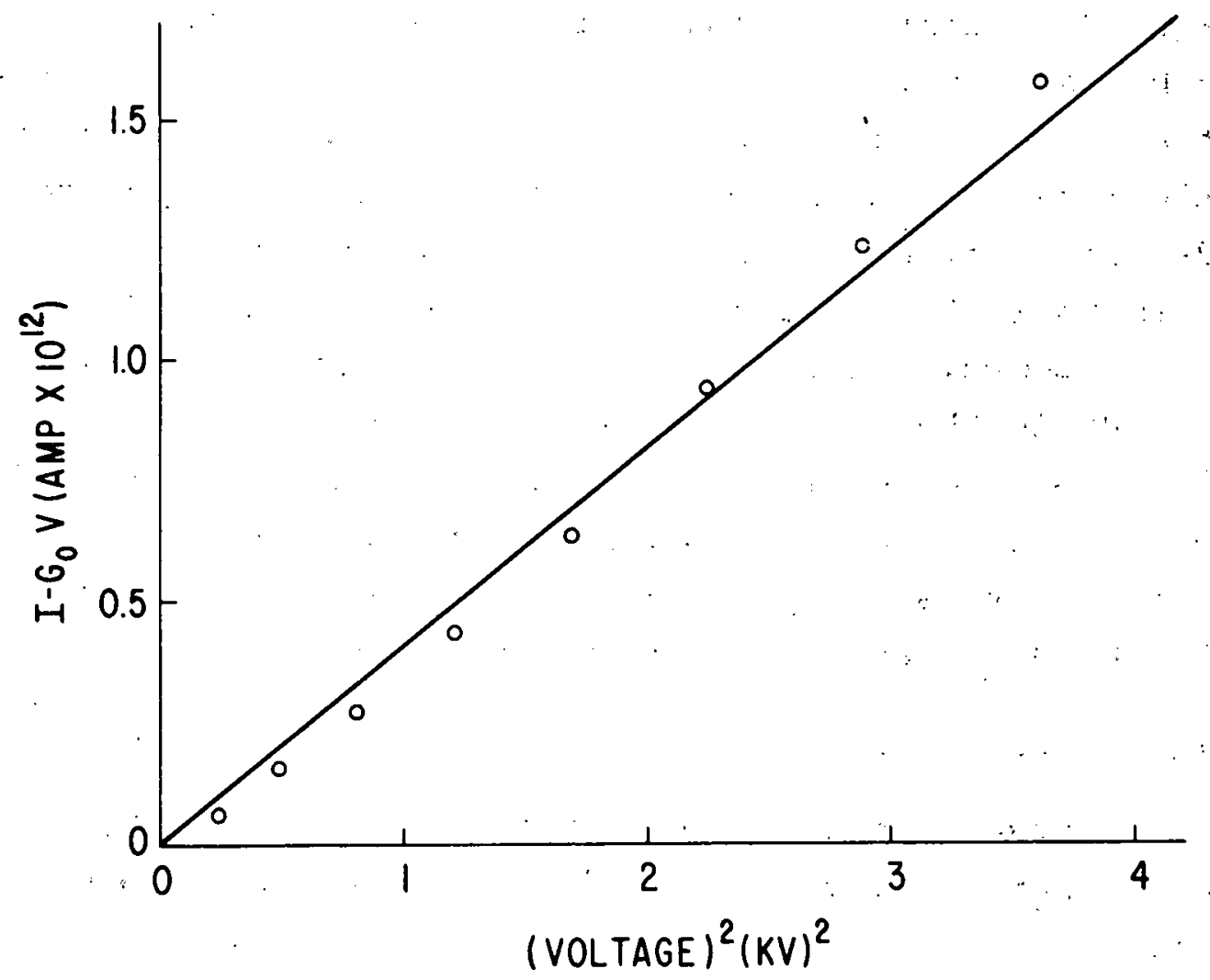

Fig. 19. Relation between nonlinear voltage-dependent portion of current and the square of voltage for polyethylene containing $5 \%$ sodium lauryl sulfate by weight, DC. 
Since $C \stackrel{\bullet}{=} 5$ pfd, then

$$
\Delta \tan \delta=\frac{\Delta G}{\omega C}=8.7 \times 10^{3} \sigma_{0} V
$$

for $60 \mathrm{~Hz}$. For our polyvinyl acetate-polyethylene-Pyranol-sodium oleate copolymer solution $\sigma_{0} \sim 1.8 \times 10^{-14} \mathrm{mho}^{-1}$ so that the expected increase in tand at $2 \mathrm{kV}$ is only $3.1 \times 10^{-7}$. For the polyethylene-sodium lauryl sulfate $\left(\sigma_{0} \sim 2.4 \times 10^{-14}\right.$ mo $\left.\mathrm{cm}^{-1}\right)$ the increase expected is only $4.2 \times 10^{-7}$. Above $2 \mathrm{kV}$, noise currents, presumably caused by discharges, made the results unreliable. Since losses due to dipoles were greater than $2 \times 10^{-4}$, and our maximum sensitivity in measuring tan $\delta$ was $1 \times 10^{-5}$, we could not expect to observe $60 \mathrm{~Hz}$ field grading in these polymeric systems.

The nonlinear dependences of current on voltage generally observed in this section for liquid systems for both DC and AC and for polymers with DC, strongly support the thesis that fieldenhanced dissociation is occurring. It may be noted, however, from Eq. (3-6) that the slopes of the linear plots of I vs V2 should be calculable without resort to arbitrary constants, so that the strongest test of the theory should be a comparison of the calculated value with those measured in our experiments. Even an order of magnitude agreement, considering particularly the geometric approximations we have made, should be considered as strongly supporting the model. The experimental slopes measured for $I$ Vs $V^{2}$ in the case of $D C$ and for $G$ Vs $V$ for $A C$ for both the liquid and polymeric systems are reported in Table 3-1 after division by $\sigma_{o}$. These are compared with the theoretical slopes. It is clear that these comparisons support the model we propose, even though detailed differences remain yet to be explained.

To summarize the results we have obtained in this section:

1. We have extended the field calculations of section II to predict current-voltage characteristics for point to plane geometries. 
2. Measurements in two low dielectric constant liquids with two types of conducting salts support the conclusion that fieldenhanced dissociation is occurring and that it is adequately described by the onsager theory. Thus, field grading at the point must be occurring.

3. This appears to be true both for $\mathrm{DC}$ and $60 \mathrm{~Hz} \mathrm{AC}$ for these liquids.

4. The same appears to be true for two polymeric systems containing dissociable salts for DC applied voltages.

5. Owing to the low conductivities in the polymeric systems we cannot conclude that these effects are also obtained at $60 \mathrm{~Hz}$ applied voltages.

\section{TABLE 3-1}

\begin{tabular}{|c|c|c|c|c|}
\hline Material & $\begin{array}{l}\text { DC slope } \\
\mathrm{cmV}^{-1} \times 10^{5} \\
\end{array}$ & $\begin{array}{l}\text { AC slope } \\
\mathrm{cmV}^{-1} \times 10^{5} \\
\end{array}$ & $\varepsilon$ & $\begin{array}{c}\text { Slope } \\
\mathrm{cmV}^{-1} \times 10^{5} \\
\end{array}$ \\
\hline $\mathrm{C}_{6}{ }^{\mathrm{H}} 6+\mathrm{TBAFB}$ & 6.75 & 11.5 & 2.3 & 1.72 \\
\hline $\mathrm{C}_{16} \mathrm{H}_{34}+\mathrm{AD} 48$ & 4.15 & 5.2 & 2.0 & 1.64 \\
\hline $\begin{aligned} \text { PVAP } & +5 \% \text { Na Oleate } \\
& +21 \% \text { Pyranol }\end{aligned}$ & 3.00 & -- & 3.1 & 1.93 \\
\hline $\mathrm{PE}+5 \%$ NALS & 1.71 & -- & 2.2 & 1.70 \\
\hline
\end{tabular}

a) The theoretical slope is $2 \pi A / Y^{2}$, where $A=0.175\left(\varepsilon \dot{T}^{2}\right)^{3 / 8}$ $(\mathrm{V} / \mathrm{cm})^{3 / 8}$ and $\mathrm{Y}=2.5 \times 10^{3}(\mathrm{~V} / \mathrm{cm})^{11 / 16}$. 
IV. ADDITIVES

\subsection{Experimental Methods}

\subsubsection{Materials}

\section{Preliminary Screening}

It was realized very early in this work that the solubility in polyethylene of ionic substances, such as organic salts, is very low. A preliminary screening of a series of compounds was therefore desirable to determine the characteristics of the additives which showed the highest solubility and conductivity in polyethylene. To facilitate this task, the screening was done by saturating $n$-hexane with the given compound. The compounds investigated, together with the dissipation factor measured at $1 \mathrm{KHz}$ are listed in Table 4-1.

of all the compounds tried, only the last showed sufficient conductivity and was our first choice for mixing with polyethylene. However, a general trend can be observed i.e. the conductivity increases with hydrocarbon chain length (e.g. oleate > butyrate $>$ acetate). This is reasonable since the solubility is expected to increase with increase in the hydrocarbon part of the salt. With the exception of the compounds marked with an asterisk, all the compounds were synthesized in very small quantities in our laboratories. Hence, because they were readily available, the following compounds were mixed with the polymers as representative of the above series: cetyltrimethylammonium bromide, sodium oleate and tetraheptylammonium chloride.

\section{Polymers}

Low density polyethylene type WPD 535B was obtained from ARCO

Union Carbide ethylene-vinyl acetate copolymer, type DQD 1868, contained $18 \%$ by weight of vinylacetate.

Further data are shown in Table 4-2. 
TABLE 4-1

\section{Dissipation Factor of Saturated Solutions in $n$-hexane}

Tetrabutyl ammonium oleate

0.006

Tetrabutyl ammonium butyrate

0.0003

Tetrabutyl ammonium acetate

0.00003

Tetrapenty 1 ammonium oleate

0.0004

Tetrapenty 1 ammonium butyrate

0.00011

Tetrapentyl ammonium propionate

0.0005

Tetraheptyl ammonium oleate

0.0043

Tetrahepty 1 ammonium propionate

0.00001

Tetraisoamyl picrate

0.0016

*Sodium picrate

0.00043

*Cetyltrimethyi ammonium bromide

0.00013

*Tetraheptyl ammonium chloride

0.00003

* Sodium oleate

0.0016

*Tetrabutyl ammonium fluoroborate

0.0002

$\left(\mathrm{C}_{6} \mathrm{H}_{5}\right)_{2}^{+} \mathrm{I} \mathrm{O}_{3}^{-} \mathrm{S}_{6} \mathrm{H}_{5} \mathrm{Cl}_{3}$

0.0001

$\mathrm{cl}^{-} \mathrm{CN}^{+}-\mathrm{NO}_{2} \mathrm{NO}_{2}$

0.0001

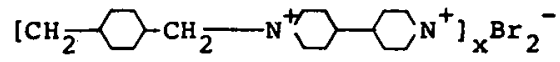

0.00002

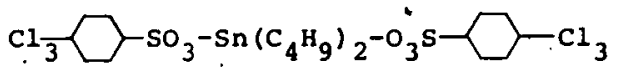

0.00003

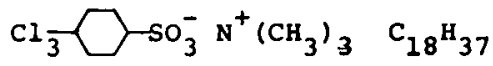

0.0000 .2

$\mathrm{C} \frac{2}{3} \bigcirc-\mathrm{SO}_{3}^{-} \mathrm{P}^{+}\left(\mathrm{C}_{6} \mathrm{H}_{5}\right)_{3} \mathrm{C}_{4} \mathrm{H}_{9}$

0.00002

$\left(\mathrm{C}_{6} \mathrm{H}_{5}\right)_{2} \mathrm{I}^{+} \mathrm{Cl}^{-}$

0.00002<smiles>C1COC2CCCC[C]2OCC[Te]OCCOC2CCCCC2OC1</smiles><smiles>CC1CCC(C(C)(C)S)CC1</smiles>

0.00004<smiles>C1CCC2OCCOCCOC3CCCC3OCCOCCOC2C1</smiles><smiles>CC1CCC(S(=O)(=O)I)CC1</smiles>

0.00005<smiles>[Z7]OC1CCOCCOCCOCCOC2CCCCC2OCC1</smiles>

$\mathrm{SCN}^{-}$

0.00006

AD 48

high conductivity

*Compounds available commercially. 
TABLE $4-2$

Characteristics of Polyethylene and Vinyl. Acetate Ethylene Copolyme.

Characteristic

Density

Melt index $\mathrm{g} / 10 \mathrm{~min}$.

Secant Modulus, psi (Stiffness)

Dissipation Factor at 10 and $100 \mathrm{KHz}$

Dielectric Constant at 10 and $100 \mathrm{KHz}$

Volume Resistivity Ohm $\mathrm{cm}$
Test Method

ASTM-D-1505068

ASTM-D-1238-65T

ASTM-D-638

ARCO-Polymers $\mathrm{W}-101-3$

ARCO-Polymers W-101-3

ARCO-Polymers

PA 144
$\underline{P E} \quad \underline{P V A P}$

$0.916-0.9190 .940$

$2.7-3.5 \quad 2.5$

5,000

$.0005(0.0013) * 0.006$ *

$2.35(2.33) * 2.85 *$

$10^{.15}\left(>10^{15}\right) * \quad>10^{15}$ *

*Measurements made in our laboratory

The ionomer obtained from E.I. DuPont de Nemours \& Co. was surlyn A 1555. This is a 5\% sodium salt of the acrylic aciäethylene copolymer.

\section{Commercial Additives}

Lithium (LiSt), sodium (NaSt) and potassium (KSt) stearates as well as stearic and myristic acids and sodium oleate were supplied by the witco Chemical Co. Sodium lauryl sulfate (NaLS), cetyltrimethylammonium bromide (CTAB) and tetraheptylammonium chloride were obtained from Eastman Organic Chemicals. Tetrabutylammonium fluoroborate (TBAFB) was supplied by Southwestern Analytical Chemicals. Four oil-soluble sulfonates were obtained from the Lubrizol Co. Some pertinent characteristics supplied by the manufacturers are listed in the Table 4-3.

1 "Ethyl" Distillate Conductivity Additive AD48, a polymeric amine salt formulation in xylene was obtained from the Ethyl co. Due to its proprietary nature the structure of this salt is unknown. However, from vapor pressure measurements a molecular weight of 2100 was obtained. Standard measurements for carbon, 
TABLE $4-3$

Characteristics of Lubrizol Oil-Soluble Sulfonates

$\begin{array}{cccc}\text { Lubrizol } & \text { Lubrizol } & \text { Lubrizol } & \text { Lubrizol: } \\ 52 & 74 & 796 & \# 25352 \mathrm{E}\end{array}$

$\begin{array}{lcccc}\text { Specific Gravity } & 0.965 & 1.14 & 1.05 & 1.28 \\ \text { Sodium } \% \text { wt } & - & - & 3.2 & 19.1 \\ \text { Calcium \% wt } & 2.9 & 12.0 & - & - \\ \text { Sulfur \% wt } & 3.15 & 1.6 & 4.0 & 1.37 \\ \text { Total Base Number } & 24 & 300 & - & 440 \\ \text { Viscosity @ } 210^{\circ} \text { F, Sus } 200 & 170 & 2600 & 321\end{array}$

hydrogen and nitrogen indicated that the compound contained 75\% C, $11.5 \% \mathrm{H}$ and $2.4 \% \mathrm{~N}$. Moreover, no halogen could be detected by $x$-ray fluorescence spectroscopy. Assuming the difference to' be oxygen, these facts led to the following general formula for the molecule of AD 48 .

$$
\mathrm{C}_{131.7^{\mathrm{H}}} \cdot 241^{\mathrm{N}} 3.59^{\mathrm{O}} 14.3
$$

Ferrocene was obtained from Aldrich Chemical Co:

All compounds were used without further purification.

Non-Commercial Additives

Sodium stearate and tetramethylammonium stearate ( $\left(\mathrm{CH}_{3}\right)_{4} \mathrm{St}$ ) were prepared by reaction of stearic acid with the corresponding... base. The agreement between the data obtained with this sodium stearate and that obtained commercially is very good (see below).

The $\alpha$-bromostearic acid was obtained by bromination of stearic acid as described by S.A. Birnbaum et al. (6) I3 ${ }^{3}$ NMR analysis indicated the purity to be better than $90 \%$. 


\subsubsection{Mixing-Pressing}

$44 \mathrm{gm}$. of the polymer was introduced into the mixing chamber of a Haake Brabender and kneaded for $5 \mathrm{~min}$. at $170^{\circ} \mathrm{C}$. The desired amount of additive, usually 5\% by weight, was then added to the polymer and mixed for another $5 \mathrm{~min}$.

The pressing was performed at $170^{\circ} \mathrm{C}$ on a wabash compression molding machine. The procedure was as follows. The desired amount of material was put into a mold which in turn was placed between the hot platens of the molding machine. $5 \mathrm{~min}$. were allowed to elapse for temperature equilibration. Then a low pressure of $1600 \mathrm{psi}$ was applied for $2 \mathrm{~min}$, followed by a high pressure of 5 tons for $2 \mathrm{~min}$. After pressure release, the mold was removed and placed between the platens of a cold press where it was pressurized at 10 tons for $5 \mathrm{~min}$. Samples of two different thicknesses, but of the same area were formed: 3 " $\times 3 "$ x 0.1 " and $3 " x 3^{\prime \prime} \times 0.02 "$.

\subsubsection{Measurements \\ Dielectric Properties}

The occurrence of field grading depends to a large extent on sufficient ionic conductivity of the material studied, and therefore it is essential to have a measurement of this quantity. There are two ways of obtaining this information; first by direct measurement of the DC resistivity, and second by measurement of the loss tangent. In the latter case one minimizes DC polarization effects. If the dielectric loss is mostly due to ionic conductance we have:

$$
\tan ^{-1} \delta=\omega C R
$$

A plot of $\tan ^{-1} \delta$ versus $\omega C$ should be linear with a slope of $R$. This value of the resistance leads immediately to the value of the AC conductivity. The measurements of the DC resistance and tans were performed using a guarded cell with parallel plane electrodes. The measuring electrode had a circular area of 
$12.6 \mathrm{~cm}^{2}$. The DC resistance was measured with a General Radio 1644-A-Megohm Bridge. The maximum resistance measurable with this bridge is $10^{15}$ ohms: The measurements were made 1 minute after voltage application, at the following voltages: 10,20 , $50,100,200 ; 500$ and $1000 \mathrm{~V}$. The loss tangent, and hence AC conductance, was measured oñ a General Radio 1615-A Capacitance Bridge operating at frequencies between $50 \mathrm{~Hz}$ and $10 \mathrm{KHz} . "$ Unfortunately, at room temperature the resistivity of the sample was often too large to be measured." However the resistivity.is:" expected to follow an Arrhenius dependence on 'temperature:

$$
\rho=\rho_{0} \exp \left(\frac{\Delta E}{R T}\right)
$$

where $\Delta \mathrm{E}$ is the activation energy in cal/mole, $\mathrm{R}$ is the universal gas constant $\left(1.99 \mathrm{cal} / \mathrm{mole}^{\mathrm{O}_{\mathrm{K}}}\right.$ ) and $\mathrm{T}$ is the temperatures in degrees Kelvin. It is then convenient to carry out measurements of the resistivity at higher temperatures and from plots of ln $\rho=$ versus $\mathrm{T}^{-1}$ to obtain the activation energy: By extrapolation one may calculate the resistivity at room temperature. We have measured the DC resistance and the tand of the sample at four different temperatures, i.e., 25, 55, 65 and $85^{\circ} \mathrm{C}$.

\section{Treeing Measurements}

Our main interest, of course, is to find whether, due to field grading, additives increase the treeing inception voltage. The procedure as well as the equipment for the measurement of these voltages was described in Part I of our report.

We have also shown in Part I that tree inception and breakdown voltages are time dependent and hence we developed a fixed schedule for the measurements. Since a cable is normally run at stresses much below those corresponding to such voltages, it was of interest to check the effectiveness of additives in preventing treeing for longer times and lower voltages.

In order to shorten data gathering time we ran ten samples: in pàrallel." The sample configuration was similar to that dis- 
cussed in Part I. The cell is shown in Fig. 20. After mounting the samples the cell is immersed in fluorochemical liquid, FC 43 (Part I, Sec. 4.2), in a Pyrex tube. Early results suggested that failure in one sample initiated trees in others, probably because of voltage surges. To minimize this we inserted two megohm resistors in series with each sample to limit the current at breakdown. When a sample failed, the voltage was automatically removed and a clock recorded the time. At this time samples showing treeing were also removed before the voltage was reapplied. The samples were initially subjected to a voltage of $15 \mathrm{kV}$ for about one week, after which it was raised to $20 \mathrm{kV}$ until all samples failed.
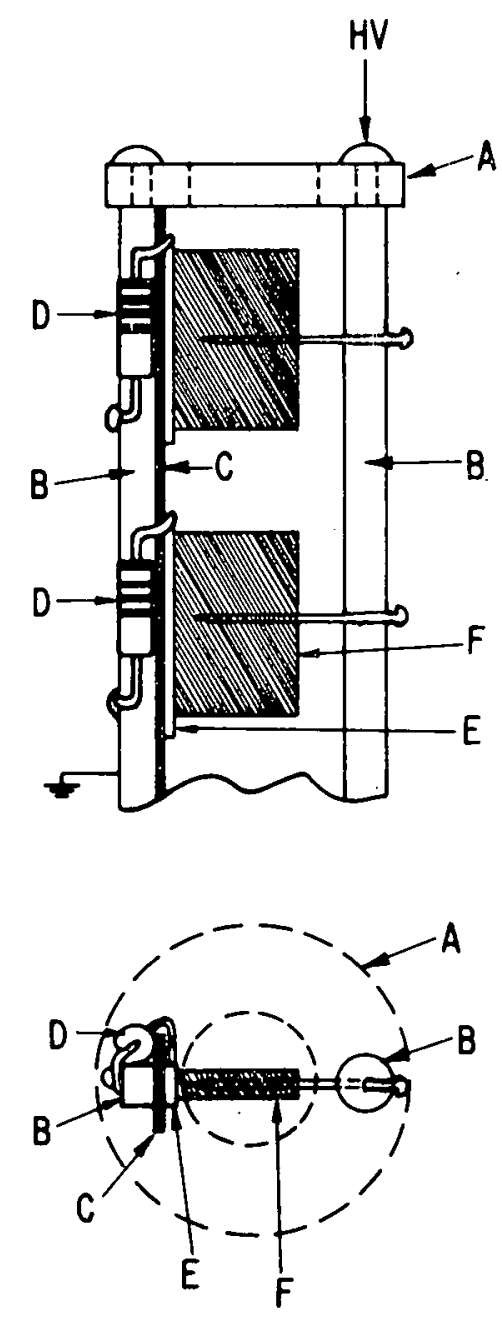

Fig. 20 Cell for long-time tree inception voltage measurements A-Teflon spacer; B-brass supports; C-Textolite spacer; D-2 megohm resistor; E-copper electrode; F-sample 


\subsection{Results}

\subsection{Typical electrical parameters}

Figures 21, 22, and 23 illustrate the type of data obtained from the measurements described above.: The sample used in the example is $5 \%$ by weight $\alpha$ bromostearic acid in polyethylene.

Figure 21 shows the plot of the 1 n of the DC resistance measured at four different voltages (namely 50, 100, 500, and $1000 \mathrm{~V}$ ) as a function of reciprocal of absolute temperature. As expected from Eq. (4-2) this plot is linear and an activation energy of $21 \mathrm{kcal} / \mathrm{mole}$ is obtained. Such an activation energy is not unreasonable in view of that measured for conduction by alkali chlorides in cellulose, acetate above the glass temperatures, $(29 \mathrm{kcal} / \mathrm{mole}){ }^{(7)}$. Figure 22 illustrates the behavior of the loss tangent with frequency at four different temperatures. At low frequencies there is a large increase of $\tan \delta$ with temperature. This is due to the decrease in resistivity, with increasing

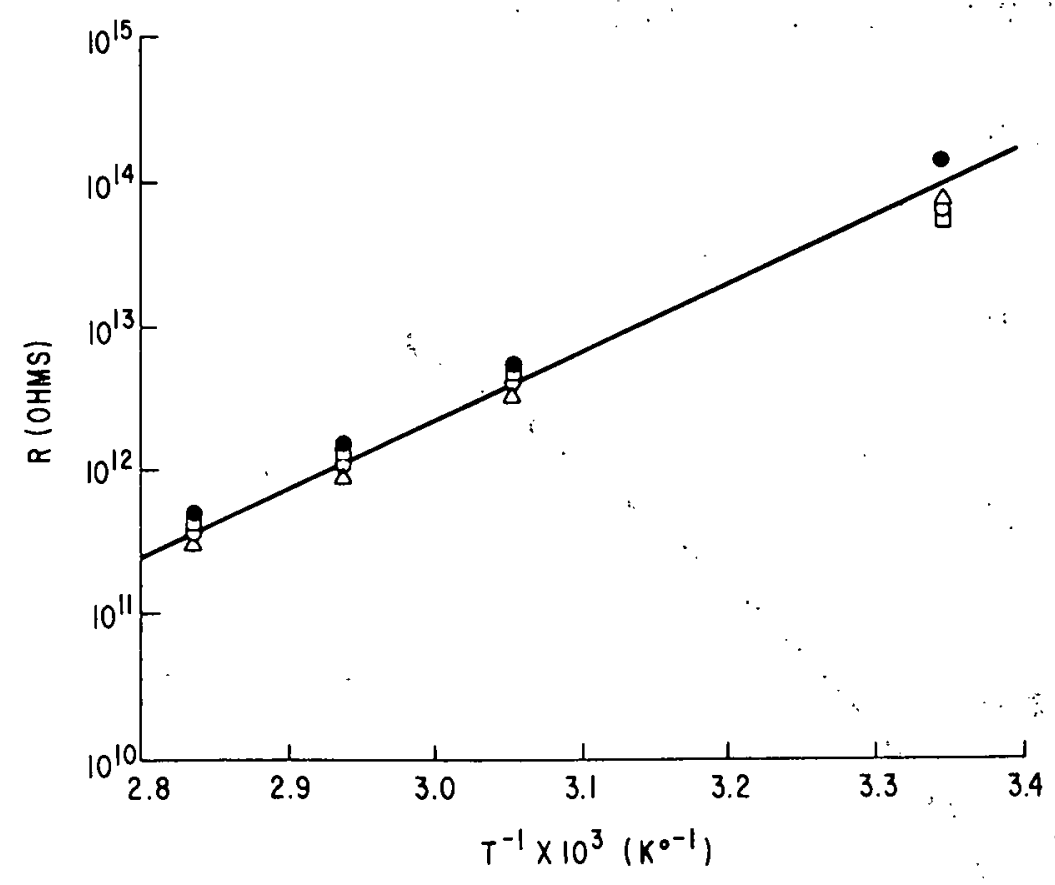

Fig. 21 Semilog plot of $R$ versus $T^{-1}$ according to Eq. 4.2 for polyethylene containing $5 \%$ by weight of $\alpha$-bromostearic acid at four different voltages. (O) $50 \mathrm{~V}$, (D) $100 \mathrm{~V}$ : (0) $500 \mathrm{~V}$ and $(\Delta) 1000 \mathrm{~V}$. 


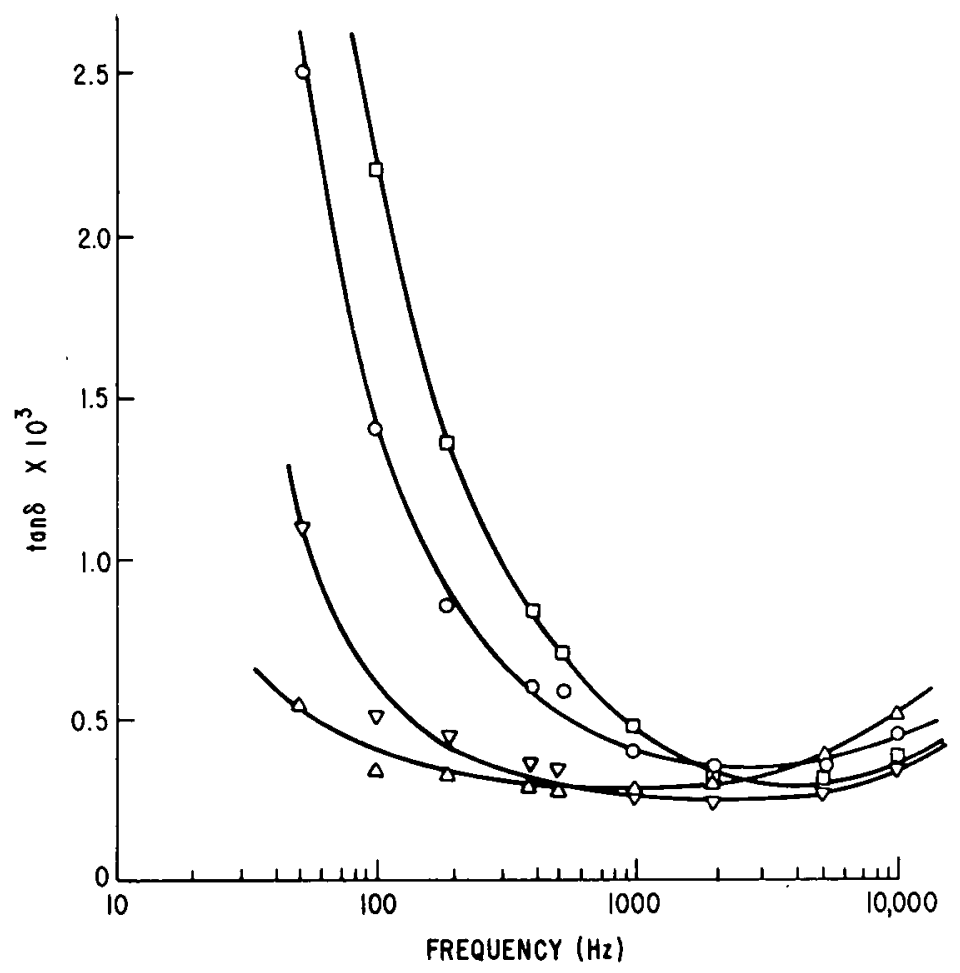

Fig. 22 Loss tangent as a function of frequency for polyethylene containing $5 \%$ by weight of $\alpha$-bromostearic acid at four different temperatures: $(\Delta) 25^{\circ} \mathrm{C}$, (D) $54^{\circ} \mathrm{C},(0) 68^{\circ} \mathrm{C}$ and (口) $85^{\circ} \mathrm{C}$.

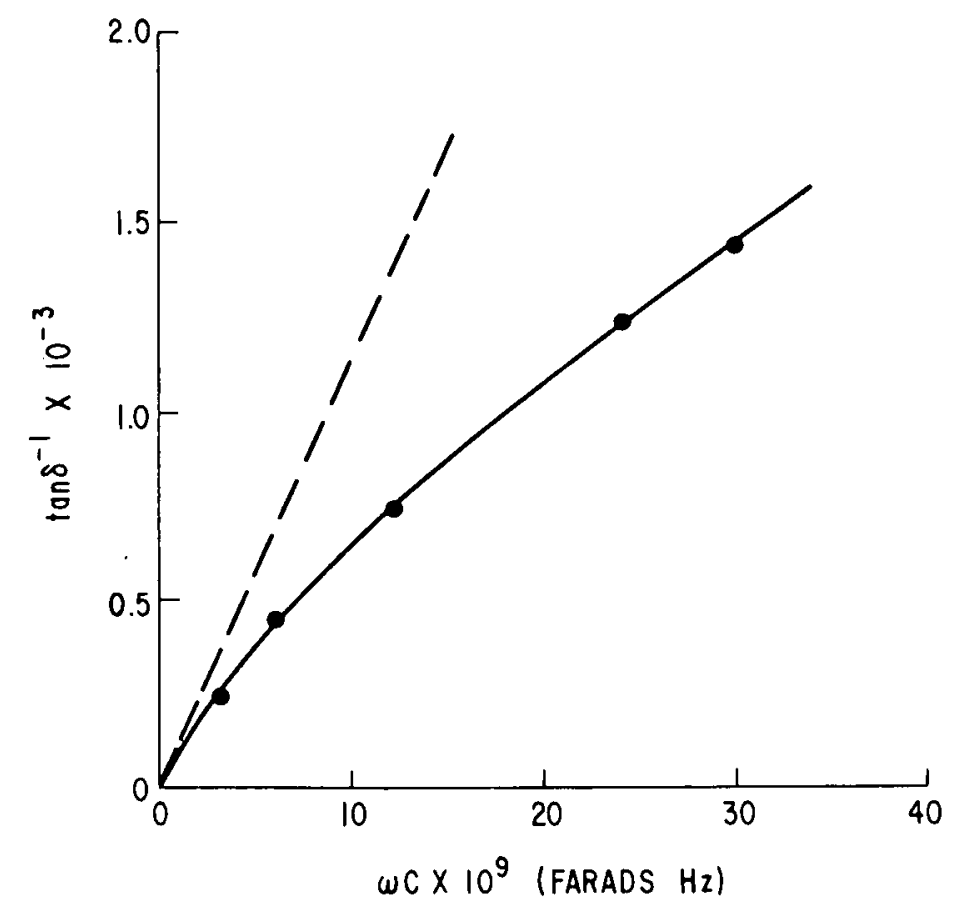

Fig. 23 Plot of $\tan ^{-1} \delta$ at $85^{\circ} \mathrm{C}$ versus $\omega C$ according to Eq. 4-1. The initial slope indicated by the dashed line. gives $\rho_{A C}=5.5 \times 1012 \Omega \mathrm{cm}$. 
temperatures. As indicated by Eq. (4-1) a plot of the value of $\tan ^{-1} \delta$ versus $\omega C$ should yield $R$ provided the loss is due to ionic conductivity. This is illustrated in Fig. 23 for data obtained at $85^{\circ} \mathrm{C}$. The dependence on $\omega \mathrm{C}$ departs rather seriously from linearity at the higher frequencies due to larger contributions of dipole losses. However the initial slope of this line should give a fairly good estimate of $R$ and hence $\rho$. Such a slope (dashed line in Fig. 23) gives for $\rho_{A C}=5.5 \times 10^{12} \Omega \mathrm{cm}$ which compares well with the value of $\rho_{D C}=1.5 \times 10^{13} \Omega \mathrm{cm}$ obtained from DC measurements at the same temperature. All, the data reported below were obtained from measurements shown in Appendix 2 .

\subsubsection{Preliminary results on additives}

Due to the difficulty of optical monitoring of polyethylene samples, some preliminary tests were carried out using vinylacetate-ethylene copolymer (PVAP) where tree growth can be observed readily. The dielectric data are shown in Table $4-4 .$.

With the exception of myristic acid, the activation energies are reasonable for ionic species diffusing in the polymer (7) In the case of myristic acid "marbling" (two phases)" could be observed and the low activation energy may be related to the motion of ions in the conducting phase. Agreement between DC and AC resistivities is fairly good. However, the room temperature resistivities are rather high. In the hope of increasing the conductivity we investigated the effect of incorporating ionic sites onto polymer molecules themselves. For this purpose we studied a $5 \%$ sodium substituted acrylic acid-ethylene copolymer (Ionomer). The resistivity, however, turned out to be fairly high as shown in Table 4-4. In the case of polyethylene the myristic acid shows again a low activation energy possibly associated with conducting paths. AD48, on the other hand, is characterized by a very large activation energy which is not surprising considering the large size of the ions involved. Unfortunately the room temperature resistivities are very large even in the presence of Pyranol. 
TABLE $4-4$

\section{Electrical Data on Polymer-Additive Mixtures}

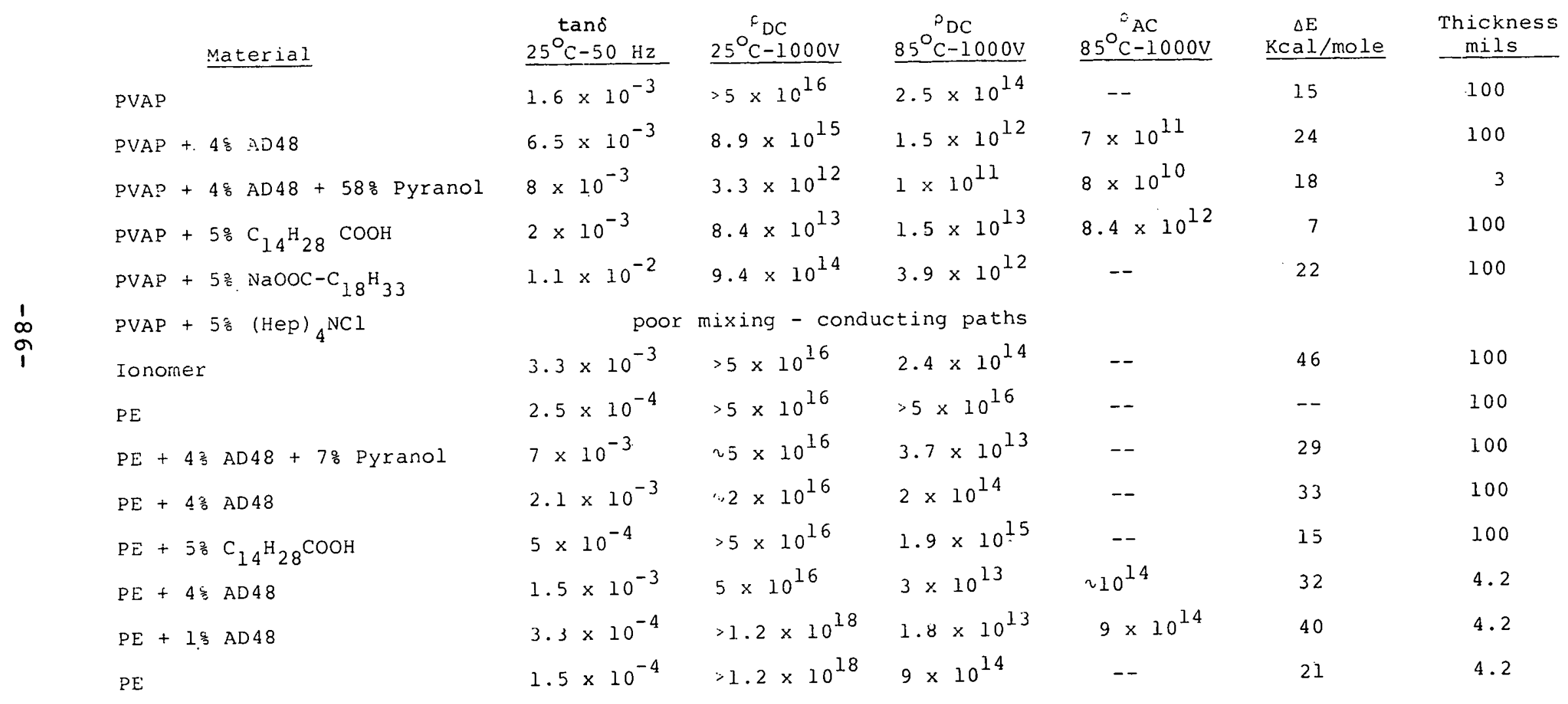


Tree inception voltage measurements have been carried out on these compounds using the standard schedule described in Part I, Sec. 4.2, and the values are reported in Table 4-5. The needles were inserted into the sample without heating, resulting in the presence of a void. A tree appeared at low voltage in every case. In the polyethylene-myristic acid combination, the needle was inserted into the sample and then heated to prevent void formation. The result was a very large increase in the tree inception voltage. In order to avoid the uncertainties. connected with the presence of voids having uncontrolled dimensions we conducted the remainder of our experiments with molded needles.

\subsubsection{Molded needle results}

Results are presented in this section on samples which had molded needles for tree inception measurements. Table 4-6 presents the electrical characteristics of all the samples studied. In many cases samples of two different thicknesses were studied in an attempt to measure more accurately the DC

TABLE 4-5

Tree Inception Voltages for Samples with Cold Inserted. Needies

Material

$$
\underline{v_{t}(k V)}
$$

PVAP

PVAP $+4 \%$ AD48

PVAP + 4\% AD48+58\% Pyranol

PVAP + 5\% Myristic Acid

PVAP + 5\% Na Oleate

Ionomer

PE

$\mathrm{PE}+4 \% \mathrm{AD} 48$

$\mathrm{PE}+5$ 응 Myristic Acid

*Average of 4 samples

\section{$10(4)$ *}

7

26 (no void) 
TABLE $4-6$

\section{Electrical Data on Polyethylene-Additive Mixtures}

Material

PE

$P E+5 \%$ LiSt

$\mathrm{PE}+5 \%$ NaSt

$\mathrm{PE}+5 \% \mathrm{KSt}$

1
$\infty$
1

\section{$25^{\circ} \mathrm{C}-5$}

$2.5 \times 10^{-4}$

$4.2 \times 10^{-4}$

$4 \times 10^{-4}$

H $7.5 \times 10^{-4}$

W $5 \times 10^{-3}$

W $1.1 \times 10^{-3}$

$8.5 \times 10^{-4}$

$4.4 \times 10^{-4}$

$7.5 \times 10^{-3}$

$9 \times 10^{-4}$

$5.5 \times 10^{-4}$

$1 \times 10^{-3}$

$1.6 \times 10^{-2}$

$6.8 \times 10^{-3}$

$6 \times 10^{-4}$

$5 \times 10^{-4}$

$1.1 \times 10^{-3}$

$8 \times 10^{-4}$

$7 \times 10^{-4}$

\section{${ }^{\rho} \mathrm{DC}$}

$25^{\circ} \mathrm{C}-1000 \mathrm{~V}$

$>5 \times 10^{16}$

$>5 \times 10^{16}$

$>2.5 \times 10^{17}$

$>5 \times 10^{16}$

$>5 \times 10^{16}$

$>2.5 \times 10^{17}$

$>5 \times 10^{16}$

$>2.5 \times 10^{17}$

$22.3 \times 10^{16}$

$>2.5 \times 10^{17}$

$2.7 \times 10^{15}$

$1.6 \times 10^{15}$

$6 \times 10^{13}$

$2.4 \times 10^{16}$

$>5 \times 10^{16}$

$>5 \times 10^{16}$

$>5 \times 10^{16}$

$>5 \times 10^{16}$

$>2.5 \times 10^{17}$

\section{${ }^{\rho} \mathrm{DC}$}

$\frac{85^{\circ} \mathrm{C}-1000 \mathrm{~V}}{16}$

$>5 \times 10^{16}$

$6.2 \times 10^{14}$

$9.4 \times 10^{14}$

$1.3 \times 10^{15}$

$9.4 \times 10^{14}$

$9.9 \times 10^{14}$

$9.5 \times 10^{14}$

$2.5 \times 10^{14}$

$1.0 \times 10^{14}$

$6.4 \times 10^{14}$

$1.5 \times 10^{13}$

$8.3 \times 10^{12}$

$2.1 \times 10^{11}$

$1.3 \times 10^{15}$

$6 \times 10^{14}$

$1.5 \times 10^{14}$

$6.1 \times 10^{14}$

$9.5 \times 10^{15}$

$5 \times 10^{15}$
$\rho_{A C}$

$85^{\circ} \mathrm{C}-1000 \mathrm{~V}$

$--$

$--$

$-$

$-$

$--$

$--$

$--$

$--$

$--$

$-$

$5.5 \times 10^{12}$

$1.2 \times 10^{13}$

$1.8 \times 10^{11}$

$--$

$--$

$--$

$-$

$--$

$-$
$\Delta \mathrm{E}$ Kcal/mole

-

23

21

28

29

29

21

25

20

$\checkmark 40$

21

21

12

13

23

23

$\therefore 27$

↔21

235
Thickness mils

100

100

20

100

100

20

100

20

100

20

100

20

100

100

100

100

100

100

20 
conductivity at room temperature. As can be seen from the table the agreement between values obtained on both samples is quite satisfactory. For the stearates although tan is reasonably low the resistivity is high. It should also be pointed out that visual examination of the samples indicated very poor solubility of the stearates in polyethylene. The activation energy, however, is that expected for molecular ion diffusion in polyethylene. Tetramethyl ammonium stearate showed signs of decomposition upon mixing and pressing. $\alpha$-bromostearic acid showed a markedly increased conductivity. This is not surprising since the substitution of an a hydrogen by an electronegative atom such as $\mathrm{Br}$ increases the dissociation constant of the molecule by decreasing the coulombic attraction between the ionic constituents. Again the agreement between different thickness samples as well as between AC and DC measurements is fairly good. The sample containing NaLs showed a very large conductivity, unfortunately probably due to conducting paths within the sample caused by poor solubility. This was visually observed and is further borne out by the low activation energy $(12 \mathrm{Kcal})$ of the resistivity. This is also the case for cetyltrimethylammonium bromide which showed extremely poor mixing with polyethylene. The series of Lubrizols behaves electrically like the stearates. However, the Lubrizols exhibited much better solubility than did the stearates. This latter fact can be understood because of the greater similarity between the polyethylene and Lubrizols than the stearates. Indeed the Lubrizols are very long chain hydrocarbons with a sulfonate group.

In Table 4-7 are reported the tree inception voltages $\left(V_{t}\right)$ and breakdown voltages $\left(V_{b}\right)$ for the components referred to in Table 4-6, with the exception of CTAB which, as mentioned previously, showed too poor a solubility in polyethyiene. The Na Lubrizols (L796 and L25352E) increase $V_{t}$ and $V_{b}$ to some extent. This is better illustrated in Fig. 24 where the normal probability distribution of $V_{t}$ for Lubrizol $25352 \mathrm{E}$ is compared to that of polyethylene. There is a definite increase both in $V_{t}$ and $V_{b}$ : 
Tree Inception and Breakdown Voltage for Sample with Molded

Needles ${ }^{\mathbf{a}}$

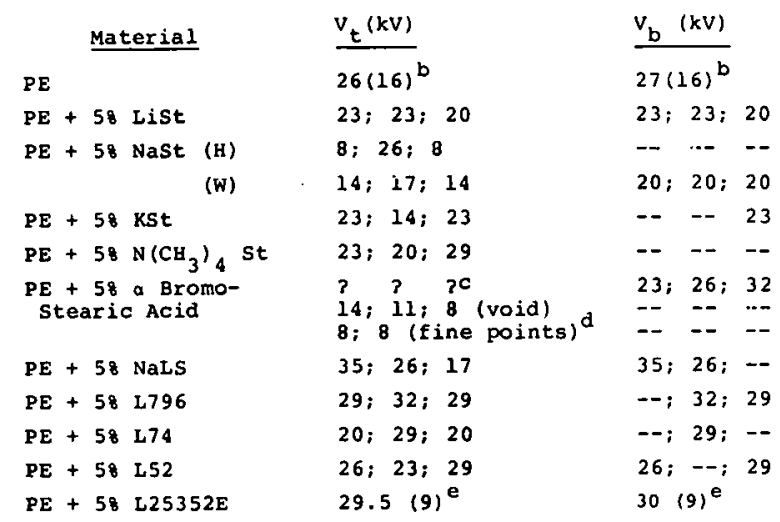

a Dashed lines indicate sampies not run to breakdown

b Average of 16 samples

c No treeing observed

d Chemically etched needles. Radius $<5 \mu \mathrm{m}$

e Average of 9 samples

H Prepared in our laboratory

W Witco

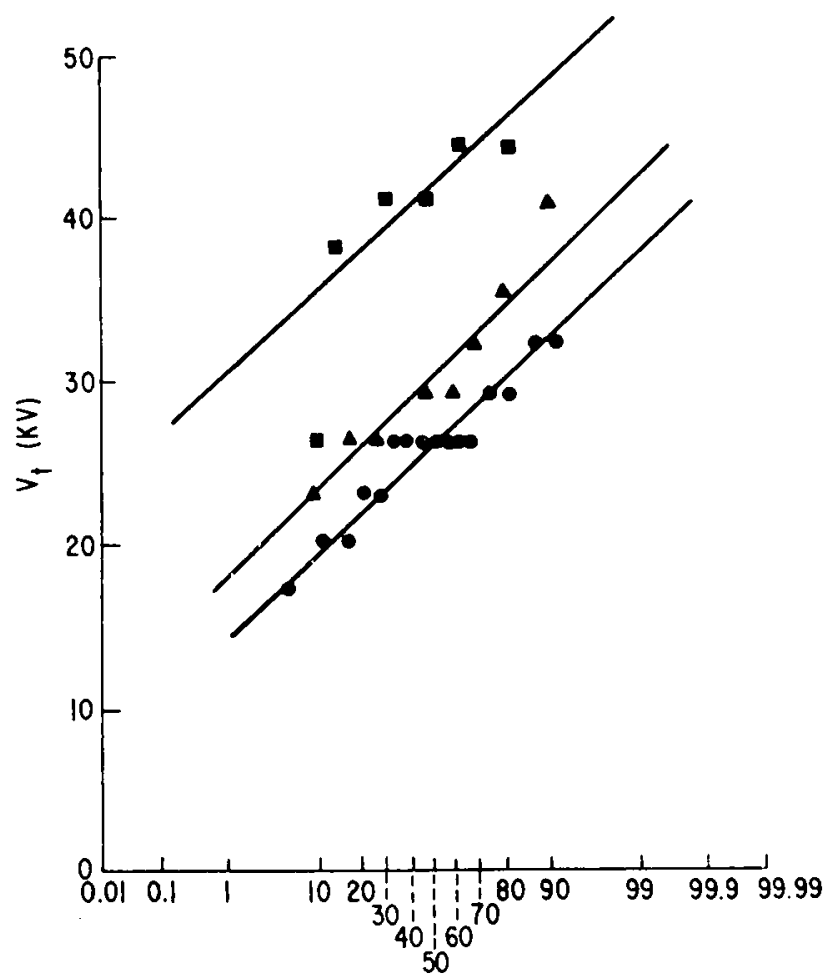

Fig. 24 Normal probability distribution plots of tree inception voltage in (O) polyethylene and (A) polyethylene $+5 \%$ L25352E, and of breakdown voltages in (U) polyethylene $+5 \%$ ferrocene. The latter represent minimum values since treeing failed to occur. 
29.5 and 30 for L25352E versus 26 and 27 for pure polyethylene. on the other hand the stearates perform very poorly: they actually decrease both $v_{t}$ and $v_{b}$. The sample containing $\alpha-$ bromostearic acid showed a very interesting behavior. With molded needles the voltage was increased till breakdown but no tree growth was observed. The average value of $\mathrm{V}_{b}$ is that for pure polyethylene $(27 \mathrm{kV})$. In order to check further for tree growth we prepared three samples with voids by cold needle insertion and two by insertion of hot needles with extremely fine points $<5 \mu \mathrm{m}$. In all cases tree growth occurred as indicated in Table 4-7. Obviously more data on this system as well as on the ones containing $\mathrm{L} 796$ and L25352E, would be desirable for a more meaningful statistical interpretation.

\section{4. $2.4 \alpha$-Bromostearic Acid}

As part of an extension to the contract, further data on treeing in PE containing 5\% $\alpha$-bromostearic acid using molded needles have been obtained. The results of our measurements, using our standard schedule, are given as a probability plot in Fig. 25. From this the average values for treeing inception and breakdown are $18.6 \mathrm{kV}$ and $23.4 \mathrm{kV}$, respectively. The standard deviation in treeing inception voltage is lower for samples containing $\alpha$-bromostearic acid than for pure polyethylene, while that for breakdown is about the same.

It should be noted that seven samples containing $\alpha$-bromostearic acid broke down showing no evidence of treeing. This occurred in four samples at $20 \mathrm{kV}$, two at $29 \mathrm{kV}$ and one at $17 \mathrm{kV}$. Breakdown in pure polyethylene, on the other hand, is invariably accompanied by tree formation. Although $\alpha$-bromostearic acid seems to prevent tree formation to some extent, the uncontrolled manner in which it operates makes unreliable any conclusions one might draw from the measurements.

The results of measurements of tree formation times in samples containing $\alpha$-bromostearic acid (PEBS) at lower voltages are shown in Table 4-8. The second column indicates the time at 


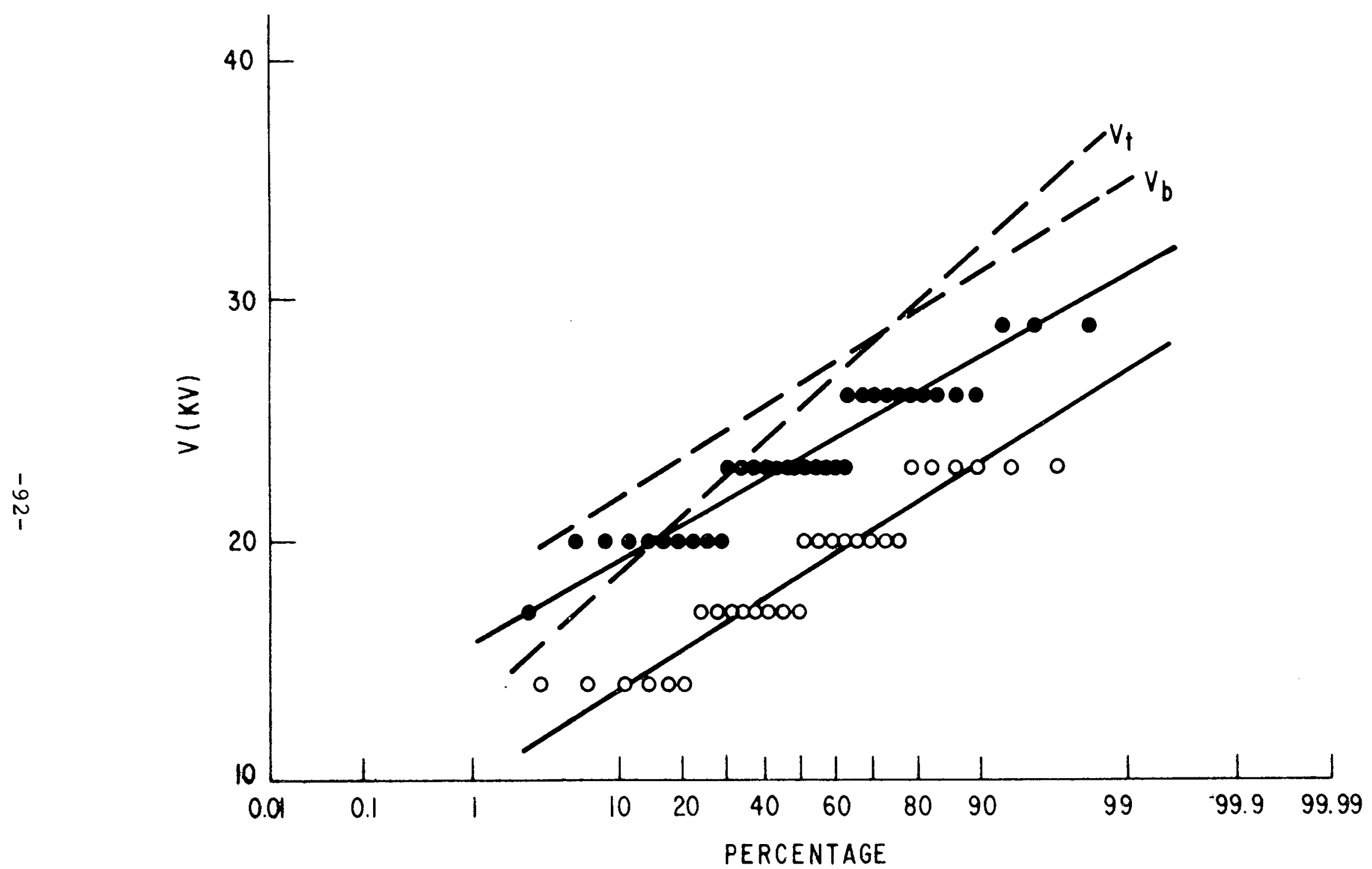

Fig. 25. Normal probability distribution plots of tree inception (o) and breakdown (•) voltages - in polyethylene $+5 \% \alpha$-bromostearic acid. Dashed lines represent the tree inception $\left(V_{t}\right)$ and breakdown $\left(V_{b}\right)$ voltage distributions in pure polyethylene. 
TABLE 4-8

Measurements of Tree Inception Times at Low Voltages

\begin{tabular}{|c|c|c|c|c|c|c|c|c|c|c|c|c|}
\hline Additive & $\begin{array}{l}\text { Time } \\
(\mathrm{hr})\end{array}$ & $\begin{array}{c}\text { Voltage } \\
(\mathrm{kV}) \\
\end{array}$ & $\underline{B}$ & $\mathrm{~A}$ & B & $\mathrm{A}$ & $\mathrm{B}$ & $\underbrace{\operatorname{lur}{ }^{2}}_{\mathrm{A}}$ & $\dot{\mathrm{B}}$ & $\begin{array}{c}\text { urs } \\
\text { A } \\
\end{array}$ & $\mathrm{B}$ & A \\
\hline \multirow[t]{2}{*}{ PEBS } & 0 & 15 & 2 . & 2 & 2 & 10 & 10 & 2 & 11 & & 10 & \\
\hline & 249 & 20 & & & & & & & & 454 & & 473 \\
\hline PEBS & 0 & 15 & 3 & 3 & 16 & 3 & 76 & 3 & 6 & 215 & 6 & 3 \\
\hline \multirow[t]{2}{*}{ L25352E } & 0 & 15 & 2 & 2 & 8 & 2 & 27 & 27 & 4 & 2 & 2 & 8 \\
\hline & & . & C & B & D & $\mathrm{C}$ & $\frac{a m p}{B}$ & $\mathrm{D}$ & $\mathrm{C}$ & B & $\begin{array}{l}: \\
\mathrm{D}\end{array}$ & c \\
\hline \multirow[t]{2}{*}{ DPPD + PEBS } & 0 & 15 & & 19 & 97 & & 19. & .19 & & 12 & 96 & \\
\hline & 151 & 20 & $663 *$ & & & 177 & & & 56.5 & & & 81 \\
\hline
\end{tabular}

$B-P E$

$A$ - PE + Additive

$C=\mathrm{PE}+2.5 \% \mathrm{DPPD}$

$\mathrm{D}-\mathrm{PE}+2.5 \% \mathrm{DPPD}+2.5 \%$-Bromostearic Acid

* - Time at which sample was removed and cut open to check for treeing. No treeing was observed.

which the voltage is raised from 15 to $20 \mathrm{kV}$. Times to failure are shown in hours in the last ten columns. Here failure refers to either treeing. or breakdown, since when a breakdown occurs samples exhibiting trees are also removed from the test cell. In general, the time between tree initiation and breakdown is approximately 1 to 2 hours.

It is noteworthy that while in most samples no differences could be attributed to the presence of $\alpha$-bromostearic acid, the three showing very much longer life did contain this additive. This perhaps is another reflection of the.presence of an uncontrolled variable in these measurements. One possibility is that unseen voids of variable sizes occur at the needle tip due to "blooming" of the acid. Another may be the presence of aggregates of $\alpha$-bromostearic acid in the high field region.

\subsubsection{Other Additives}

It may be possible to effectively shield voids present by producing a conducting layer on their surfaces. Additives for 
such a purpose have been proposed by Fujiki et al. (8), and include N, $N^{\prime}$ diphenyl-p-phenylene diamine (DPPD). However, a conducting void or conducting tree should behave as a fieldenhancing asperity. It was therefore of interest to determine whether a synergistic effect could be found using a combination of DPPD (to short the void) and $\alpha$-bromostearic acid (for field grading).

The results are shown on probability plots in Fig. 26 for the mixed additives, and Fig. 27 for DPPD alone. Included for comparison purposes are our earlier measurements on pure polyethylene. While in both cases the tree inception voltages are lowered, the breakdown voltages are about the same for DPPD alone, but are significantly higher with the mixture (50\% values 27 and $30 \mathrm{kV}$ respectively). However, measurements of time to failure shown in Table 4-8 seem to indicate that DPPD by itself is more effective in tree prevention than is the mixture.

Since the conductivity of polyethylene containing AD48 was somewhat higher than with many other additives (Table 4-4), we decided to examine treeing in this material with molded needles. The results are presented in Fig. 28. The dashed lines refer to treeing and breakdown in pure polyethylene. It is clear that while the tree inception voltage may be slightly lowered by AD48, no measurable change occurred in the breakdown voltage.

Since Lubrizol L25352E has shown an increase in breakdown voltage (Fig. 24), the long time behaviour was examined. The results are presented in Table 4-8 and indicate no positive improvement due to this additive.

\subsubsection{Polyethylene-L25352E - Pyranol System}

The dissociation constant is very sensitive to the dielectric constant of the medium. Increasing the dielectric constant should increase the zero field conductivity and hence field grading in polymeric systems in which the conductivity is insufficiently high. We have used Pyranol \#1499 $(\varepsilon=4.6)$ to increase the 


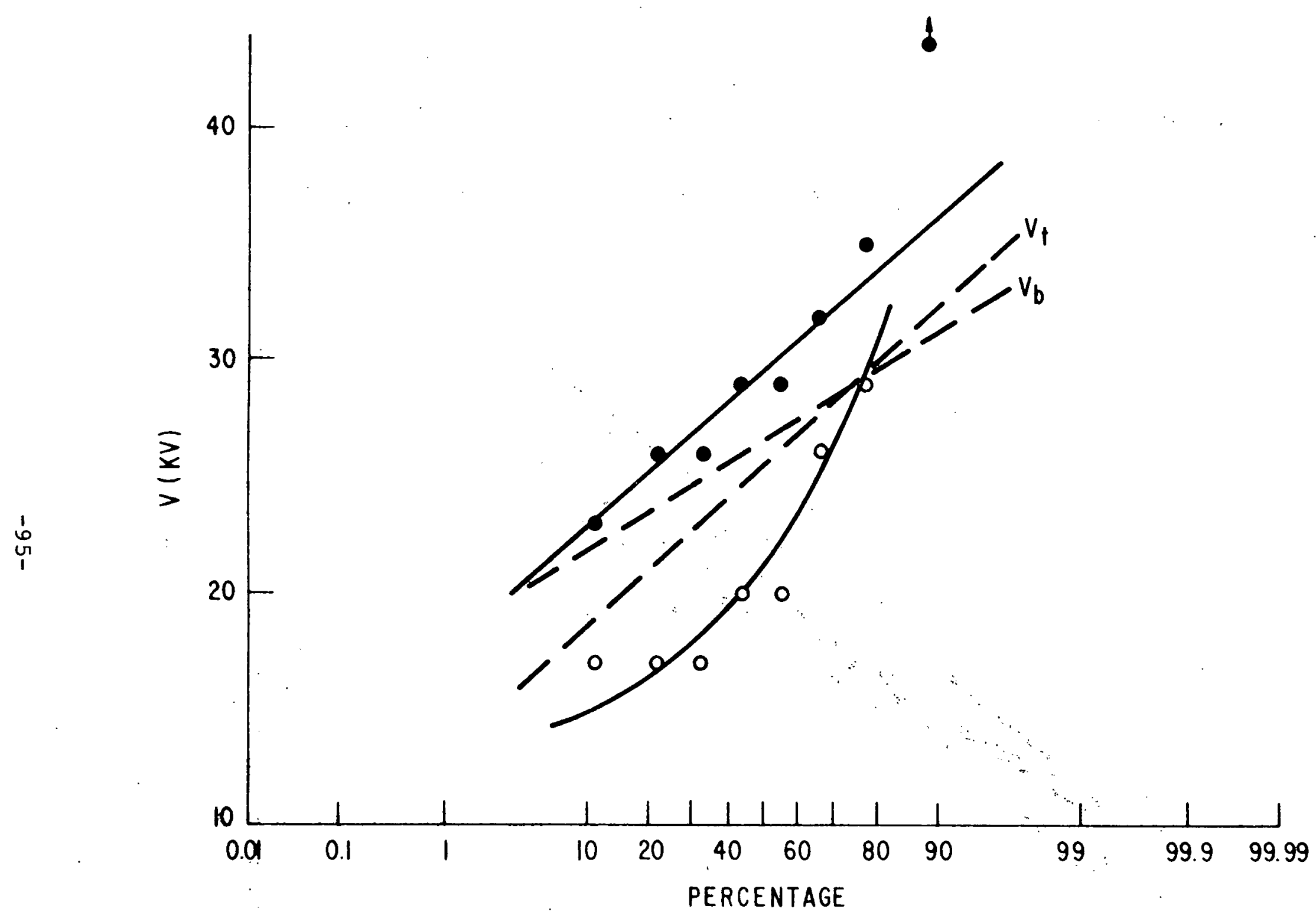

Fig. 26. Normal probability distribution plots of tree inception (o) and breakdown (•) voltages in polyethylene $+2.5 \% \alpha$-bromostearic acid $+2.5 \% \mathrm{~N}, \mathrm{~N}^{\prime}$ diphenyl-p-phenylene diamine. Dashed lines represent the tree inception $\left(V_{t}\right)$ and breakdown $\left(V_{b}\right)$ voltage distributions in pure polyethylene. The arrow indicates sample where flashover occurred before breakdown. 


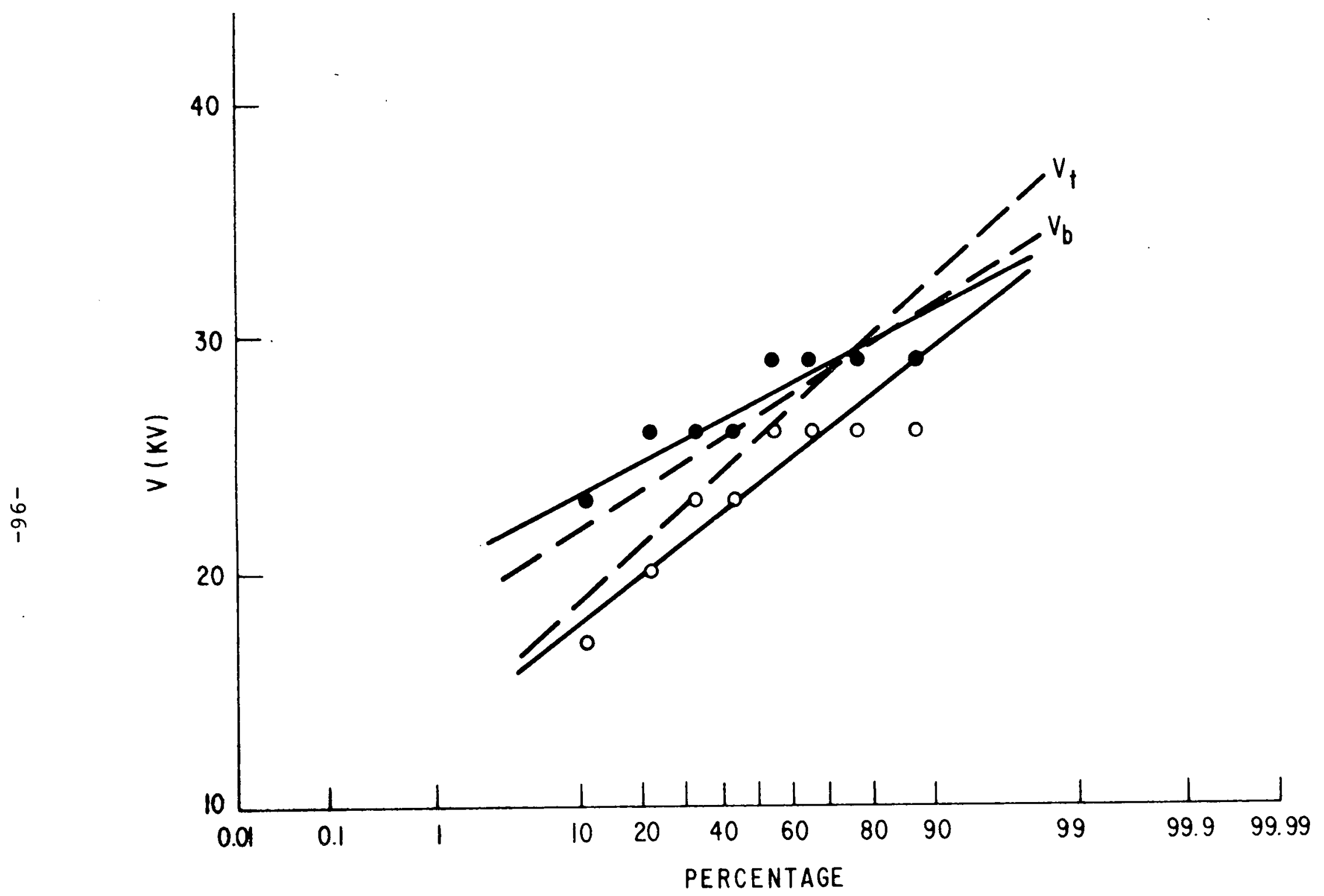

Fig. 27: Normal probability distribution plots of tree inception (o) and breakdown (•) voltages in polyethylene $+2.5 \% \mathrm{~N}, \mathrm{~N}^{\prime}$ diphenyl-p-phenylene diamine. Dashed lines represent. the tree inception $\left(V_{t}\right)$ and breakdown $\left(V_{b}\right)$ voltage distributions for pure polyethylene. 


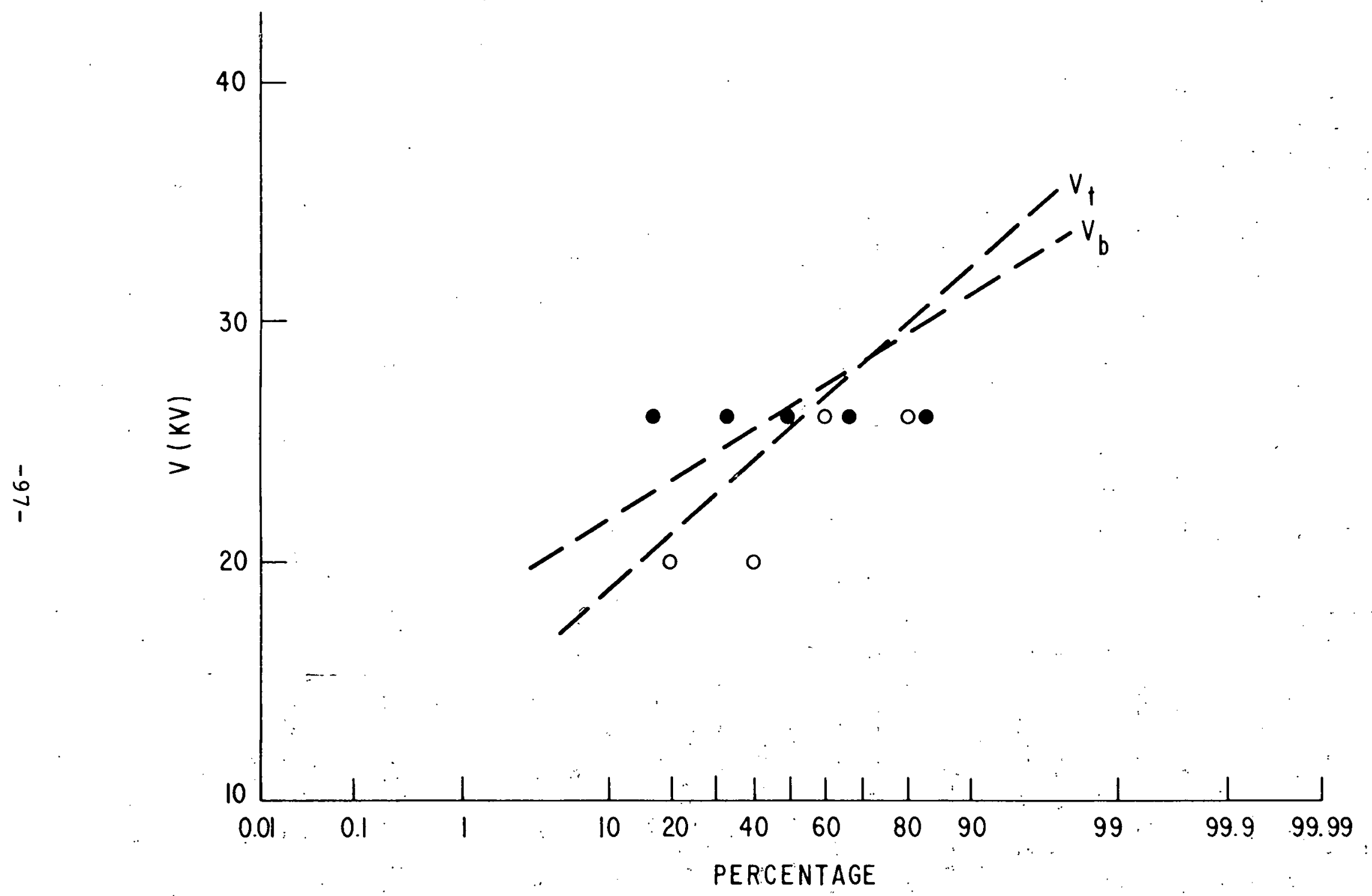

Fig. 28. Normal probability distribution plots of tree inception (o) and breakdown ( $)$ voltages in polyethylene $+5 \%$ AD48. Dashed lines represent the tree inception $\left(V_{t}\right)$ and breakdown $\left(V_{b}\right)$ voltage distributions in pure polyethylene. 
dielectric constant of the PE $+5 \%$ L25352E system, which has been shown to inhibit treeing to some extent. Two concentrations of Pyranol were used: $10 \%$ and $30 \%$ by weight. The former appears to be below the saturation swelling concentration of polyethylene, since the Pyranol did not ooze from the samples. On the other hand the 30\% Pyranol samples showed substantial oiliness suggesting that this concentration is above the equilibrium swelling concentration. As indicated in Table 4-9, the zero field resistivity decreases with an increase in the dielectric constant due to the addition of Pyranol. At $85^{\circ} \mathrm{C}$ the decrease is of the order of magnitude of 50 to 100 at $10 \%$ Pyranol. Further addition of Pyranol still reduces the resistivity, although, as expected, the very large percentage of Pyranol affects the physical properties of the material as illustrated by the decrease in the activation energy of the resistivity. At both Pyranol concentrations, treeing was completely suppressed indicating indeed that the conductivity is high enough for field grading to occur. However, the breakdown voltages of these systems, given in the normal probability plot of $\mathrm{Fig} .29$, show no improvement over the PE + 5\% L 25352E system without Pyranol represented by the solid line in Fig. 29. Possibly another mechanism of breakdown (e.g. thermal) is operative so that field grading is not observed to the full extent.

TABLE $4-9$

Electrical Data on Polyethylene - 5\% L25352E - Pyranol Mixture

Pyranol Concentration

$--$

$10 \%$

$30 \%$ $25^{\circ} \tan \delta$ $25 \mathrm{C}-100 \mathrm{~Hz}$

$4.6 \times 10^{-4}>5 \times 10^{16}$

$5.2 \times 10^{-4}$

$5 \times 10^{-4}$

$6.5 \times 10^{-4}$

$6.8 \times 10^{-3}$

$4.6 \times 10^{-3}$

${ }^{\circ} \mathrm{DC}$

$2 \times 10^{15}$
${ }^{\rho} \mathrm{DC}$

$\triangle E$ ThickKcal/ ness

mole mils

$\sim 21$

100

$2.5 \times 10^{17}$

$2.4 \times 10^{16}$

$2.5 \times 10^{14}$

$4.7 \times 10^{16}$

$7.8 \times 10^{13}$

$3.5 \times 10^{13}$

$7.4 \times 1014$

$4.0 \times 10^{13}$ $\sim 35$

24

26

18

13
20

$\underline{\varepsilon(100 \mathrm{~Hz})}$

2.36

100

2.56

20

100

2.66 


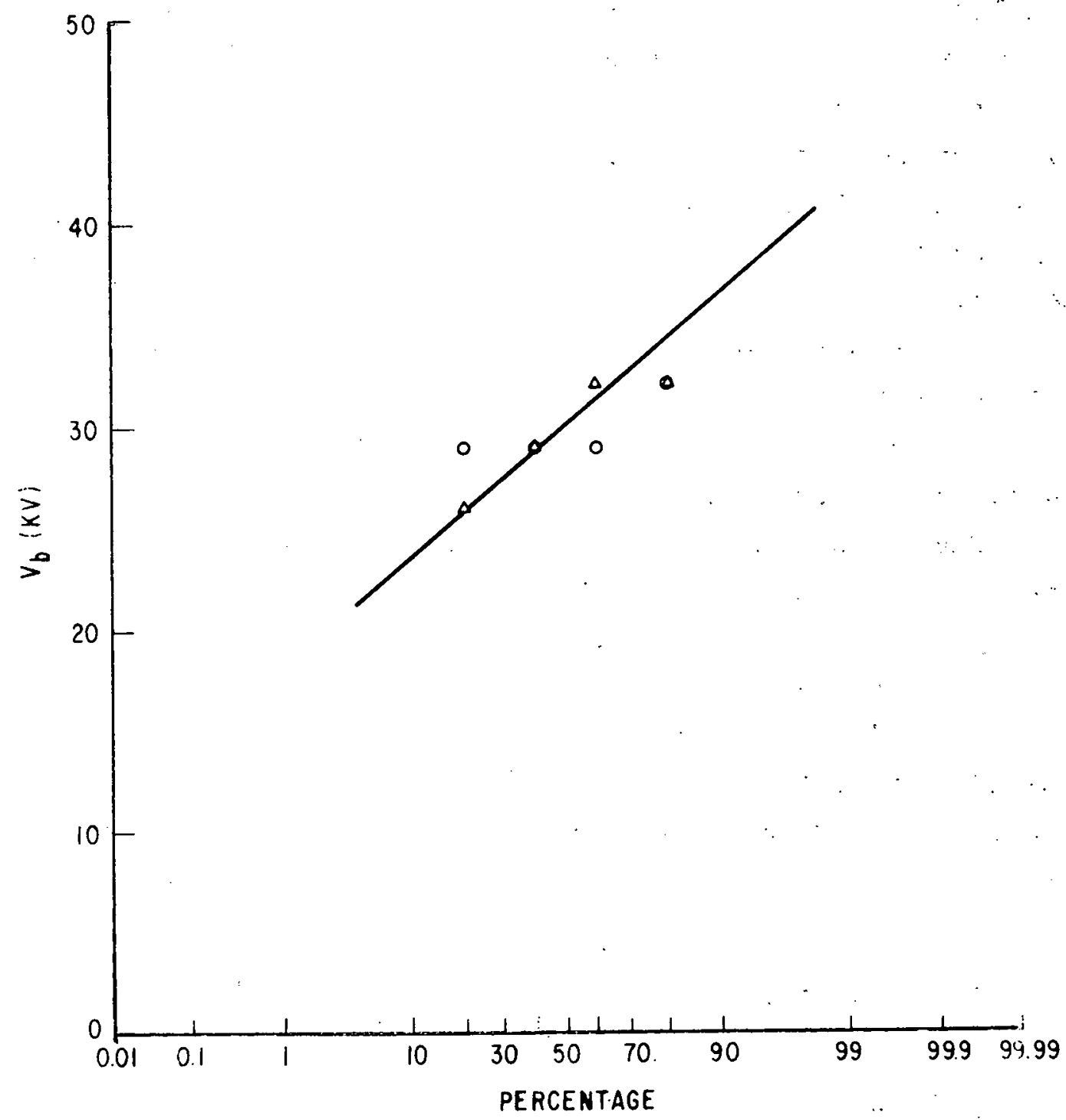

Fig. 29 Normal probability distribution plot of breakdown voltages in the polyethylene $+5 \% \mathrm{~L} 25352 \mathrm{E}$ system $(-)$ with 10\% ( $\Delta$ ) and 30\% (o) Pyranol \#1449 added. Gap: 100 mils. Radius needle: $1.5 \mathrm{mil}$.

\subsubsection{Ferrocene system}

Recently it has been shown by kato et al. "(9) that addition of ferrocene to polyethylene increased the tree initiation voltage. Ferrocene, which consists of an iron atom sandwiched between two cyclopentadiene rings, is not, at first sight, expected to dissociate into ions and hence should not be able to grade the field according to our present model. However, due to the effect on the tree inception voltage we decided to examine a $5 \%$ ferrocene- 
polyethylene system. This mixture exhibits extremely high resistivity even at $85^{\circ} \mathrm{C}$ (Table 4-10) preventing the measurement of the activation energy. The loss tangent dependence on frequency is quite different from that of other compounds studied. There is a peak at $100 \mathrm{~Hz}$ at room temperature $\left(\tan \delta=4 \times 10^{-4}\right)$ which moves towards higher frequencies as the temperature is raised. The $50 \mathrm{~Hz}$ loss tangent at $85^{\circ} \mathrm{C}$ is extremely small. When this sample is submitted to our standard treeing procedure, the voltage can be raised to very high values ( $\sim 40 \mathrm{kV}$ ) at which point flashover appears to occur somewhere in the system leaving the sample apparently intact. However, when the voltage is raised again (linearly in about $30 \mathrm{sec}$ ) breakdown occurs at voltages ranging between 20 and $25 \mathrm{kV}$. It should be stressed, however, that under no conditions, even with voids, has tree growth been observed in the ferrocene-containing samples. The values of the "first flashover" are presented in the normal probability distribution plot of Fig. 24 . One can readily see a dramatic improvement relative to the values pertinent to pure polyethylene.

TABLE 4-10

Electrical Properties of $5 \%$ Ferrocene in Polyethylene

$\begin{array}{cccc}\begin{array}{c}\tan \delta \\ 25^{\circ} \mathrm{C}-50 \mathrm{~Hz}\end{array} & \frac{25^{\circ} \mathrm{C}-1000 \mathrm{~V}}{2 \times 10^{-4}} & \frac{85^{\circ} \mathrm{C}-1000 \mathrm{~V}}{\text { P }} & \frac{\begin{array}{c}\text { Thickness } \\ \text { mils }\end{array}}{3 \times 10^{16}} \\ 3 \times 10^{-4} & >2.5 \times 10^{17} & \sim 1 \times 10^{16} & 20\end{array}$.

The photographs in Fig. 30 show the complete absence of treeing prior to breakdown in the polyethylene-ferrocene system. The rather tortuous breakdown path shown at $24 \mathrm{kV}$ on the second increase of voltage is characteristic of this material. The somewhat larger discharges than are normally present are believed to be background in the system caused by previous flashovers on the spacers and not due to discharges in the sample. 


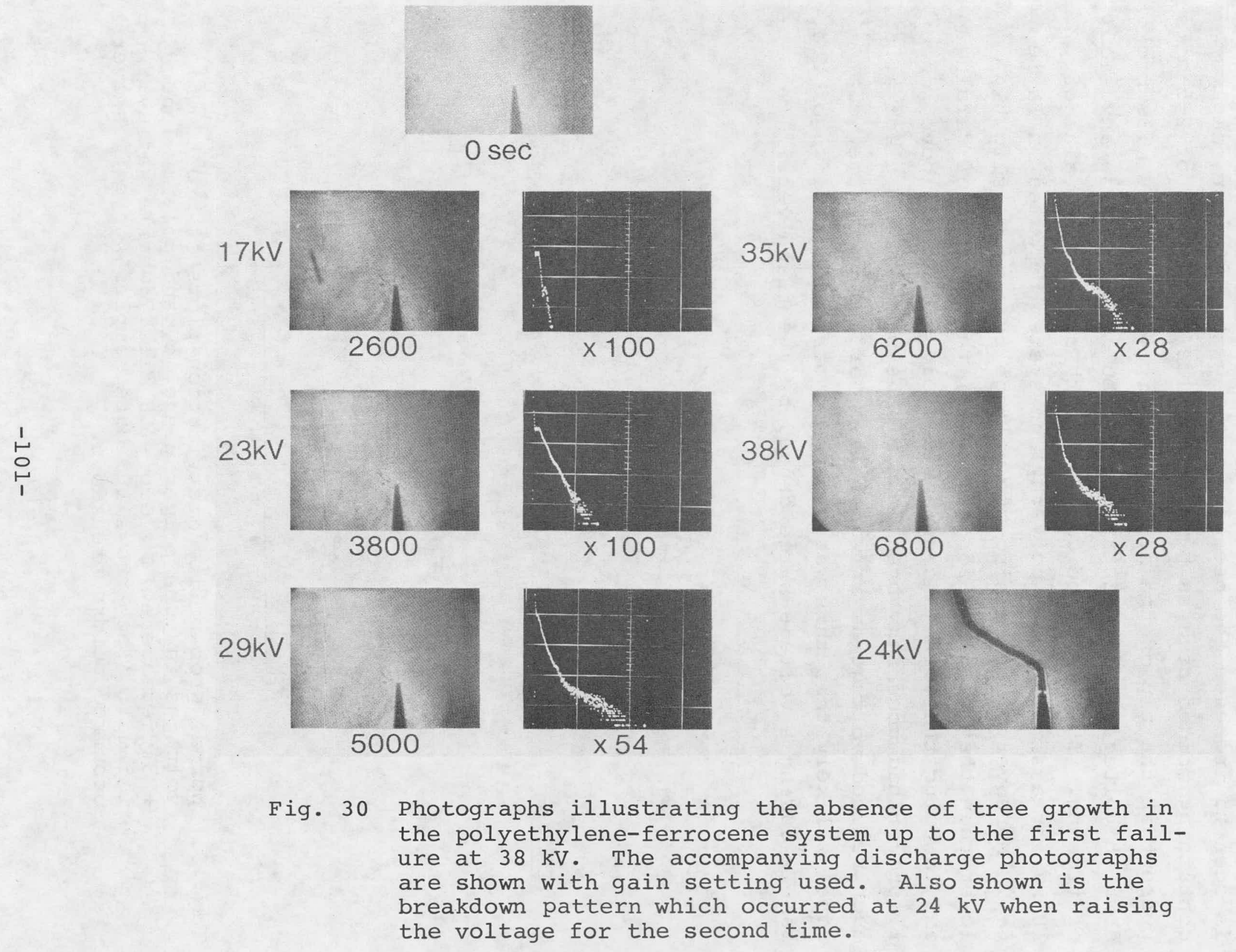


In order to have a better measure of the effect we studied samples with a smaller gap i.e. 60 mils. Breakdown or flashover voltages are shown in the normal probability plot of Fig. 31, together with the data pertinent to pure polyethylene. Ferrocene is seen to increase the breakdown voltage by $100 \%$ for samples containing molded needles. Three samples with voids of $2-5 \mathrm{mils}$ present at the tip of the needle and with gaps of $100 \mathrm{mils}$ broke down at voltages of 17,20 and $38 \mathrm{kV}$, but did not show any sign of treeing. This is to be compared with an average value of $10 \mathrm{kV}$ for a similar pure polyethylene system reported in Table 4-5. Moreover, when raising the voltage to a value slightly lower than that required for flashover, the process of raising and lowering the voltage can be repeated. As noted above, once flashover had occurred, breakdown of the sample took place at a much reduced voltage as it was raised for a second time. It appears likely that this was caused by partial breakdown of the sample during a voltage surge caused by the flashover.

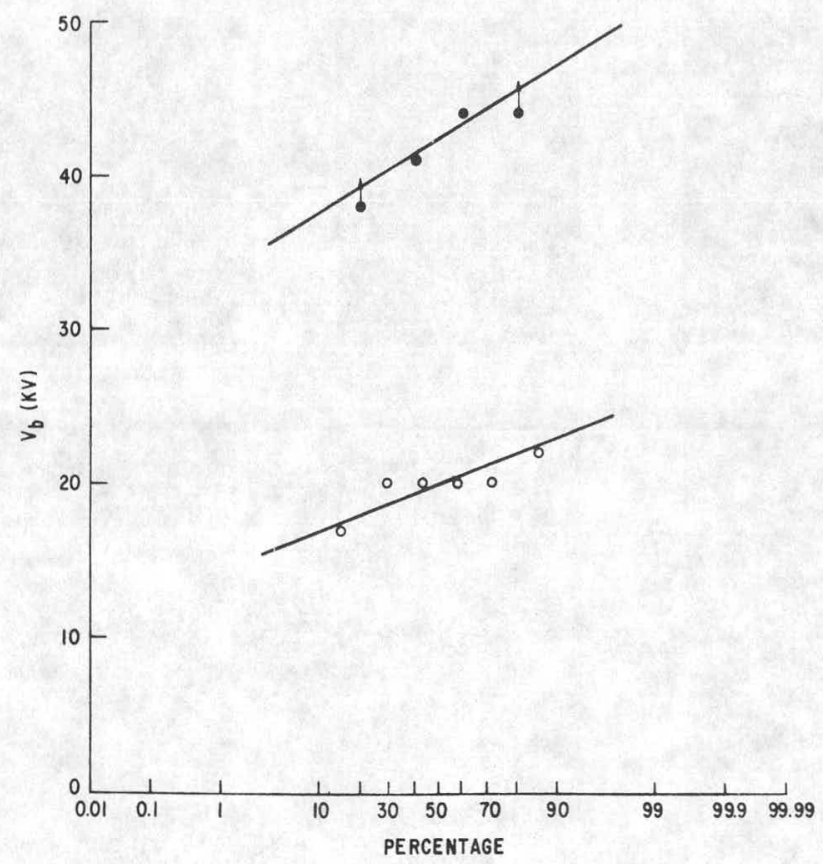

Fig. 31 Normal probability distribution plots of breakdown voltages in pure polyethylene, 0 , and polyethylene $+5 \%$ ferrocene., . Gap $=60 \mathrm{mils}$. Needle radius: $1.5 \mathrm{mil}$. The arrows indicate samples where flashover occurred before breakdown. 


\subsubsection{Conclusions}

From the above results the following conclusions can be drawn.

1. Solubility of ionic substances in polyethylene increases with increasing size of the hydrocarbon portion of the molecule.

2. The conductivity in polyethylene increases when the dissociation constant of the additive is increased (such as obtained by $\alpha$-bromination of stearic acid).

3. Although $\alpha$-bromostearic acid and sodium lubrizols inhibit treeing in the molded needle samples, we were unable to completely suppress treeing with the ionic additives used to date (cf however, Ferrocene below).

4. The sodium-containing sulfonates L796 and L25352E (Lubrizols) increase both the tree inception and breakdown voltages by about 10\%. $\alpha$-bromostearic acid, however, does not increase these voltages.

5. The addition of a mixture of $N, N^{\prime}$ diphenyl-p-phenylene diamine and $\alpha$-bromostearic acid decreases the tree inception voltage but raises the breakdown voltage by about $10 \%$.

6. The long time test of tree inception times indicates that the effects of $\mathrm{L} 25352 \mathrm{E}$ and $\alpha$-bromostearic acid and its mixture with $N, N^{\prime}$ diphenyl-p-phenylene diamine; are marginal. However, the $\mathrm{N}, \mathrm{N}^{\prime}$ diphenyl-p-phenylene diamine alone increases the treeing time by a factor of about 60 .

7. Addition of Pyranol 1499 to a polyethylene-L25352E system substantially increases the conductivity, completely suppresses treeing, but does not increase the breakdown voltage.

8. Ferrocene completely suppresses treeing and increases the breakdown strength of polyethylene by $100 \%$. Whether it is due to field grading is not clear. 


\section{FREQUENCY DEPENDENCE OF FIELD GRADING}

\subsection{Introduction}

We have demonstrated from current-voltage measurements the expected field grading phenomenon in liquids with DC and $60 \mathrm{~Hz}$ voltages and in polymers with DC voltages. However, we have been unable to demonstrate in polymers the large increase in tree inception voltage predicted by our model. One possible explanation is that the mechanism of breakdown changes when ionic additives are added, so that treeing is suppressed in favor of, for example, thermal breakdown. Another possibility is that sufficient field grading is not occurring because the large dependence of dissociation on field predicted by onsager does not occur in such viscous media as polymers at $60 \mathrm{~Hz}$. For example, the rates of dissociation may be insufficient to permit equilibrium to be established at this frequency.

In an effort to examine the latter point, we have studied the influence of frequency on the field dependence of conductivity for sinusoidal fields, first theoretically and second experimentally.

\subsection{Dissociation Kinetics in Sinusoidal Fields}

Quite generally we have an ion-pair in equilibrium with its constituent ions

$$
\mathrm{AB} \underset{\mathrm{k}_{\mathrm{r}}}{\stackrel{\mathrm{k}_{\mathrm{f}}}{\rightleftarrows}} \mathrm{A}^{+}+\mathrm{B}^{-} \quad \mathrm{K}(\mathrm{E})
$$

where $K(E)$ is the field-dependent equilibrium constant.

If we start with $n$ molar $A B$, the concentrations of $\mathrm{AB}, \mathrm{A}^{+}$ and $\mathrm{B}^{-}$at any time $\tau$ after application of a field step will be given by

$$
\begin{aligned}
& {[\mathrm{AB}]=\mathrm{n}(1-\alpha)} \\
& {\left[\mathrm{A}^{+}\right]=\left[\mathrm{B}^{-}\right]=\alpha \mathrm{n}}
\end{aligned}
$$


where $\alpha$ is the degree of dissociation at time $\tau$. When, for a given field $E$, the steady state is achieved the equilibrium constant is given by

$$
K(E)=\frac{k_{f}(E)}{k_{r}}=\frac{\left[A^{+}\right]\left[B^{-}\right]}{[A B]}=\frac{\alpha_{o}^{2} n^{2}}{n\left(1-\alpha_{o}\right)}
$$

where $k_{f}(E)$ and $k_{r}$ are respectively the forward and reverse reaction rate constants in the equilibrium $(5-1)$, and $\alpha_{0}$ is the steady state value of $\alpha$. As indicated, only the forward reaction rate is field dependent.

The rate of disappearance of $A B$ is given by

$$
\frac{-d[A B]}{d t}=k_{f}[A B]-k_{r}\left[A^{+}\right]\left[B^{-}\right]
$$

which, together with $(5-2),(5-3)$, and $(5-4)$, gives

$$
\frac{d \alpha}{d t}=k_{r}^{\alpha}{ }_{0}^{2} \frac{(1-\alpha)}{\left(1-\alpha_{0}\right)}-k_{r} \alpha^{2} n
$$

or

$$
\frac{d \alpha}{k_{r}^{\alpha}{ }_{o}^{2} n\left(\frac{1-\alpha}{1-\alpha_{o}}\right)-k_{r}{ }^{2} n}=d t
$$

This expression can easily be integrated to give after some manipulations

$$
\frac{\left(\alpha-\alpha_{0}\right)}{\alpha+\frac{\alpha_{0}}{1-\alpha_{0}}}=\mathrm{Ce}^{-t / \tau}
$$

where $C$ is an integration constant and the time constant $\tau$ is given by

$$
\tau=\frac{\left(1-\alpha_{0}\right)}{\left(2-\alpha_{0}\right){ }^{n} \alpha_{o} k_{r}}
$$


As pointed out by onsager, assuming that $\mathrm{k}_{r}$ is simply the diffusion controlled recombination rate constant of the ions, the time constant, $\tau$, is simply one-half the Maxwell time-lag $\left(\tau_{M}=\frac{\varepsilon}{4 \pi \sigma}\right)$ for the readjustment of charges in a conductor.

For a given voltage step the behavior of $\alpha$ in $\mathrm{Eq} \cdot(5-8)$ is illustrated in Fig. 32. It is seen to increase exponentially with a time constant $\tau$, towards the steady state value $\alpha_{0}$.

If one desires to obtain the behavior of $\alpha$ under an applied AC sinewave voltage, one has to go back to expression (5-6) and integrate it over such a wave. This integration is quite involved and it is much more convenient to perform a numerical integration. To do this we have rewritten Eq. (5-6) by making use of the definition (Eq. (5-9)) of the time constant $\tau$, i.e.:

$$
\frac{d \alpha}{d t}=\frac{\alpha_{0}^{2}(1-\alpha)-\alpha^{2}\left(1-\alpha_{0}\right)}{\tau \alpha_{0}\left(2-\alpha_{0}\right)}
$$

These calculations were done for a typical system i.e. $0.0005 \%$ TBAFB in benzene. The initial concentration of TBAFB is $\mathrm{n}=1.34 \times 10^{-6} \mathrm{M}$.

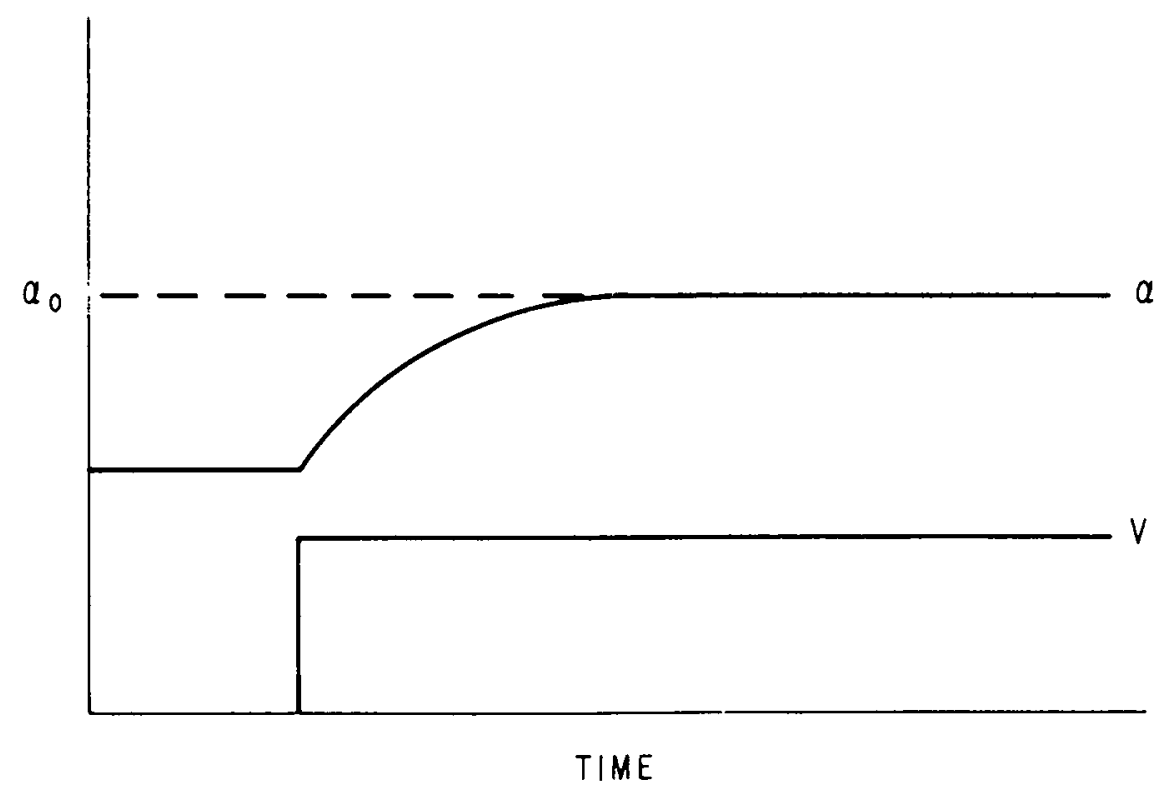

Fig. 32 Illustration of the time dependence of the degree of dissociation $\alpha$ for a voltage step such as shown in the figure. The steady state limit is $\alpha_{0}$. 
The computation was performed in the following way. First the value of $\alpha_{0}$ was evaluated. For values of $\alpha<<1$, which is always the case in our systems,

$$
\alpha_{0}=\alpha_{i}(F(b))^{1 / 2}
$$

where $\alpha_{i}$ is the degree of dissociation before the application of the field and $F(b)$ is the onsager field enhancement factor which can be computed from the power series:

$$
F(b)=1+b+\frac{b^{2}}{3}+\frac{b^{3}}{18}+\frac{b^{4}}{180}+\frac{b^{5}}{2700}+\frac{b^{6}}{56700} \ldots
$$

For a sinusoidal voltage wave:

$$
\mathrm{b}=\frac{9.636 \mathrm{~V}_{\mathrm{O}} \sin \omega t}{\varepsilon \mathrm{T}^{2} \mathrm{~L}}
$$

where $L$ is the gap length.

$\alpha_{i}$ is obtained from the initial equilibrium constant as given in Eq. $(5-4)$ i.e.

$$
\mathrm{k}_{i}=\frac{\mathrm{n} \alpha_{i}^{2}}{1-\alpha_{i}}
$$

and since $\alpha_{i}<<1$ one gets $\alpha_{i}=\left(\mathrm{K}_{i} / \mathrm{n}\right)^{1 / 2}$. The initial value of the equilibrium constant can be computed from the zero field initial concentrations of ions $\left(n_{i}\right)$ and ion pairs $(n=1.34 \mathrm{x}$ $\left.10^{-6} \mathrm{M}\right)$ i.e., $\mathrm{K}_{i}=\mathrm{n}_{i}{ }^{2} / \mathrm{n}$. Finally, $\mathrm{n}_{i}$ is obtained from the experimental zero field conductivity

$$
\sigma_{0}=n_{+} \mu_{+} e+n_{-} \mu_{-} e=2.07 \times 10^{-11} \text { mo } \mathrm{cm}^{-1}
$$

Since $\mu_{+} \simeq \mu_{-}$and $n_{+}=n_{-}, \sigma_{0}=2 n_{i} \mu$ e. With $\mu=10^{-3} \mathrm{~cm}^{2} \mathrm{~V}^{-1}$. $\sec ^{-1}$ one gets $n_{i}=2.15 \times 10^{-10} \mathrm{M}$, which gives $\mathrm{k}_{i}=3.46 \times 10^{-16} \mathrm{M}$ and in $\operatorname{turn} \alpha_{i}=1.61 \times 10^{-6}$.

The last parameter needed in order to evaluate $\tau$ is $k_{r}$ which, assuming diffusion controlled kinetics is given by 


$$
k_{r}=4 \pi r_{C}\left(D_{+}+D_{-}\right)
$$

where $D_{\text {+ }}$ and $D_{\text {- }}$ are the diffusion coefficients of the respective ions and $r_{c}$ is onsager's critical length given by $e^{2} / \varepsilon \mathrm{kT}$ i.e., the distance at which the coulombic energy between the ions equals $\mathrm{kT}$. Since $\mathrm{D}=\mu \mathrm{kT} / \mathrm{e}$, and with $\mathrm{N}$ being Avogadro's number one gets in units of $\mathrm{M}^{-1} \mathrm{sec}^{-1}$

$$
k_{r}=\frac{4 \pi e}{\varepsilon}\left(\mu_{+}+\mu_{-}\right) \mathrm{N} .
$$

For benzene at room temperature $\varepsilon=2.28$, and as indicated above $\left(\mu_{+}+\mu_{-}\right)=10^{-3} \mathrm{~cm}^{2} \mathrm{v}^{-1} \mathrm{sec}^{-1}$. This gives $\mathrm{k}_{\mathrm{r}}=4.78 \times 10^{11_{\mathrm{M}}^{-1}}$ $\mathrm{sec}^{-1}$ which is very similar to ion recombination rate constants reported in the literature ${ }^{(10)}$.

With all these parameters Eq. (5-10) can be numerically integrated for different frequencies and voltages. The dependence of $\alpha$ on frequency for a peak voltage of 1000 volts is illustrated in Fig. 33, where $\alpha$ is plotted as a function of wt for three different frequencies: 10, 50, and $100 \mathrm{~Hz}$. It is readily seen that not only does a reach its average value very rapidly ( 2 cycles at $100 \mathrm{~Hz}$ ), but also that for the frequencies studied a tends towards a common average value. This latter point is further emphasized in Fig. 34, where the average conductivity is plotted as a function of peak voltage for 5 different frequencies 10,50, 100, 1000, and $5000 \mathrm{~Hz}$. With the exception of some scatter at $1000 \mathrm{~V}$, mostly due to excessively. large integration increments, the data at the 5 frequencies are indistinguishable, indicating that the field dependent conductivity is independent of frequency. Moreover, as illustrated in Fig. 34, the conductivity varies linearly with voltage at each frequency, as would be expected in this low field regime. (It should be noted here that the conductivity $\sigma$ is directly proportional to $\alpha$ since $\sigma=2 \mu e \alpha n$ ).

It may be concluded from this analysis that according to the onsager theory the field-dependent conductivity should be the 


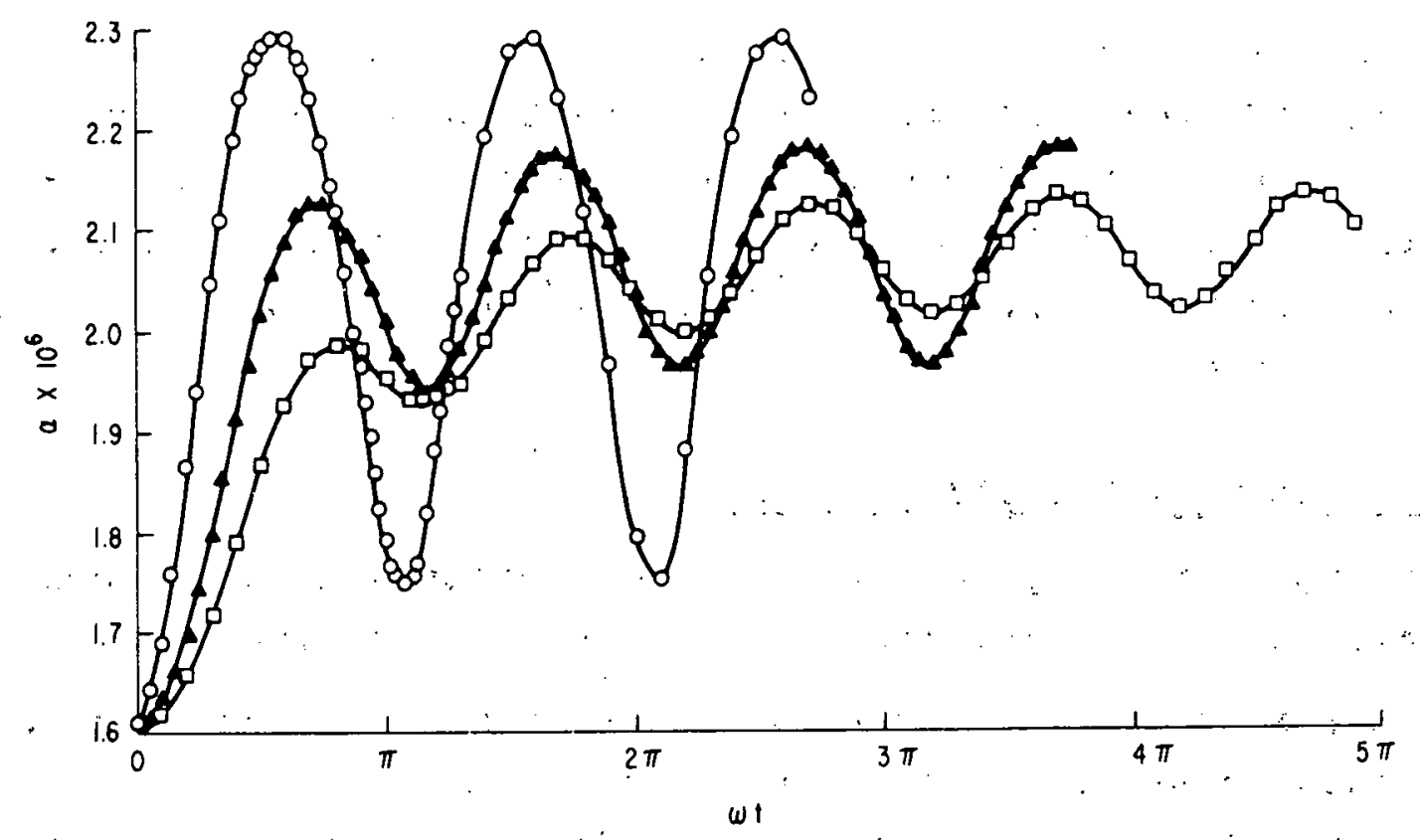

Fig. 33 Dependence of the degree of dissociation a on wt. for a peak voltage of $1,000 \mathrm{~V}$, calculated with $\mathrm{Eq} \cdot(5-10):(0)$ $10 \mathrm{~Hz}$, (L) $50 \mathrm{~Hz}$, (a) $100 \mathrm{~Hz}$.

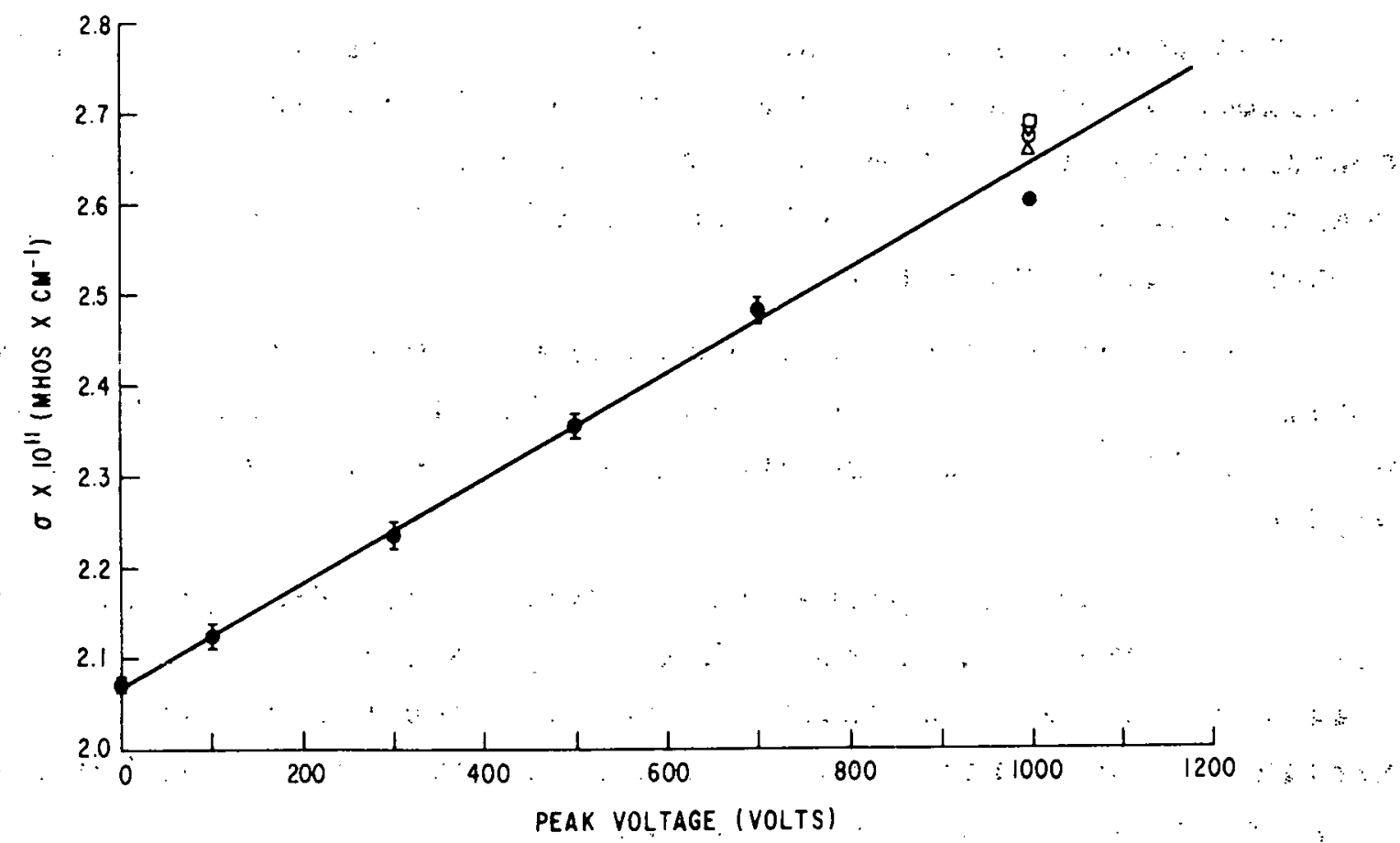

Fig. 34 Voltage dependence of the average conductivity obtained from calculations like "those illustrated in Figure 33. The limits indicate the total variation over a frequency range from 10 to $5000 \mathrm{~Hz}$. At 1,000 volts (e) $10 \mathrm{~Hz},(\Delta)$ $50 \mathrm{~Hz}$, (O) $100 \mathrm{~Hz},(\nabla) 1,000 \mathrm{~Hz}$, () $5,000 \mathrm{~Hz}$. 
same in AC fields as it is in DC fields. In both cases it is only necessary for sufficient time to be allowed for a steady state to be reached. The rate at which this occurs is determined to within a factor of two by the Maxwell relaxation time, which for conductivities of $10^{-13^{3}}$ mho $\mathrm{cm}^{-1}$ will be of the order of a few seconds.

\subsection{Experimental Results in Liquids}

To experimentally verify these conclusions we have examined the field dependent conductivity as a function of frequency.

Preliminary to an investigation in polymers we used conductance measurements in point-plane geometries in liquid solutions in the hope of observing a dispersion in the field-assisted dissociation at frequencies above $60 \mathrm{~Hz}$. We have chosen these higher frequencies since, with liquids, grading occurs at DC and $60 \mathrm{~Hz}$ so that any dispersion would be expected here.

The conductance measurements were performed in the cell described previously using a conventional schering bridge in conjunction with a power supply capable of several kV at frequencies up to $10 \mathrm{KHz}$. A General Radio $1232 \mathrm{~A}$ detector having a tunable filter was used.

The solution used in the measurement was $0.15 \%$ by weight Ethyl AD48 in n-hexadecane. The zero field conductivity was $\sigma_{0}$ $=1.03 \times 10^{-9}$ mho $\mathrm{cm}^{-1}$ measured with the General Radio Megohm Bridge.

The results are presented in Fig. 35 for frequencies ranging between 70 and $2000 \mathrm{~Hz}$ and voltages up to $1 \mathrm{kV}$ (RMS). Unfortunately, since the dissipation factor decreases with increasing frequency, the sensitivity of the measurements was inadequate at frequencies higher than $2000 \mathrm{~Hz}$.

If field grading is operative at a given frequency the conductance should increase linearly with voltage with a slope equal to $2 \pi \mathrm{A} \sigma_{0} / Y^{2}$ where $\mathrm{A}=0.175\left(\varepsilon \mathrm{T}^{2}\right)^{3 / 8}(\mathrm{~V} / \mathrm{cm})^{3 / 8}$ and $\mathrm{Y}=$ $2.5 \times 10^{3}(\mathrm{~V} / \mathrm{cm}) 11 / 16$. That this is so at all frequencies 

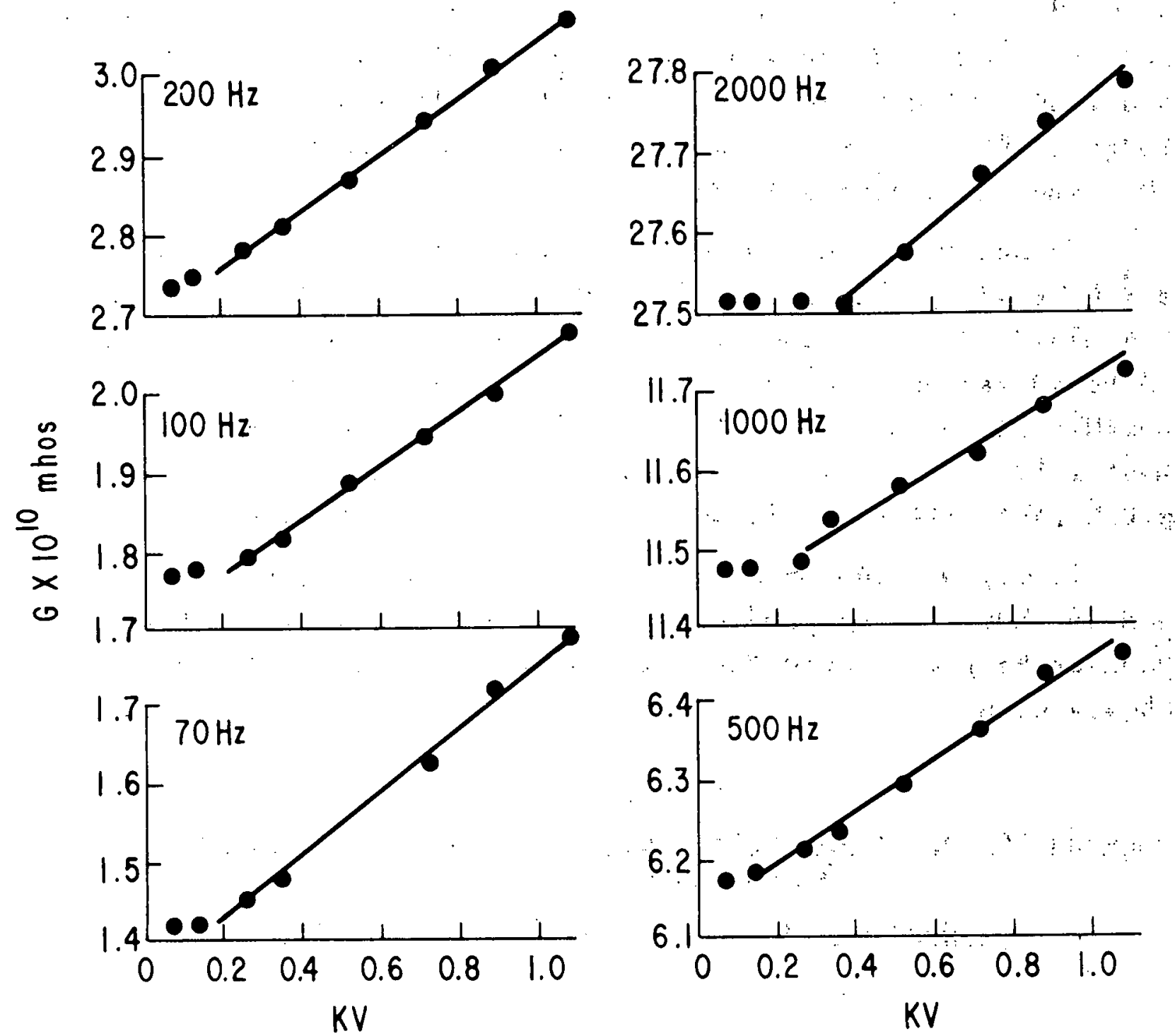

Fig. 35. Voltage dependence of the AC conductance at different frequencies, for a solution of $0.15 \%$, by weight AD 48 in $\mathrm{n}$-Hexadecane. 
studied, is readily seen in Fig. 35. The values of slope/o obtained from Fig. 35 are presented in Table 5-1 together with the values obtained previously at $60 \mathrm{~Hz}$ and from I vs $\mathrm{V}^{2}$ measurements in the DC case. The remarkable constancy of the slope confirms the analysis in sec. 5.2.

It should be pointed out here that there is an increase by a factor of approximately 20 in the zero field conductance when increasing the frequency from 70 to $2000 \mathrm{~Hz}$ (Fig. 35). This increase can be associated with dipole losses in the solution. Indeed, it is not unexpected that the very large size of the AD48 molecule as well as the large size of the solvent molecule would give rise to a dipole loss peak in this frequency range.

It may be concluded, therefore, that for liquids the onsager theory does predict the correct field dependence of conductivity in time-dependent fields, and that AC field grading should occur.

TABLE 5-1

Dependence on Frequency of the Slope obtained from Plots of Conductance Versus Voltage

\begin{tabular}{|c|c|}
\hline Frequency $(\mathrm{Hz})$ & slope $/ \sigma_{0}(\mathrm{~cm} / \mathrm{V}) *$ \\
\hline 70 & $4.28 \times 10^{-5}$ \\
\hline 100 & $3.50 \times 10^{-5}$ \\
\hline 200 & $4.11 \times 10^{-5}$ \\
\hline 500 & $3.20 \times 10^{-5}$ \\
\hline 1000 & $3.12 \times 10^{-5}$ \\
\hline 2000 & $3.41-3.97 \times 10^{-5}$ \\
\hline 60 & $5.2 \times 10^{-5}$ \\
\hline DC & $4.15 \times 10^{-5}$ \\
\hline
\end{tabular}




\subsection{Experimental Results on Polyethylene Systems}

For polymers a dispersion in the field dependent conductivity would be expected at frequencies lower than $6.0 \mathrm{~Hz}$. . Thus we constructed a voltage amplifier to be used with our. low frequency bridge $(.02$ to $10 \mathrm{~Hz} ; 0-2.5 \mathrm{kV})$. The loss tangent due to ionic conduction increases with decreasing frequency, so that the sensitivity of our measurements. would be improved. Time did, not permit completion of these measurements.

Another approach to the problem is to measure tree inception voltages at lower frequencies. To this end we have modified a DC power supply to provide a very low frequency saw tooth voltage wave. Figure 36 indicates how this is accomplished. A high voltage reversing switch is operated manually to reverse polarity at voltage zeros. For a given frequency it is necessary to alter the rate of rise for each change in voltage. We were able to vary the frequency over the range 0.005 to $0.04 \mathrm{~Hz}$. with a maximum peak voltage of $34 \mathrm{kV}$ determined by flashover at the switch.
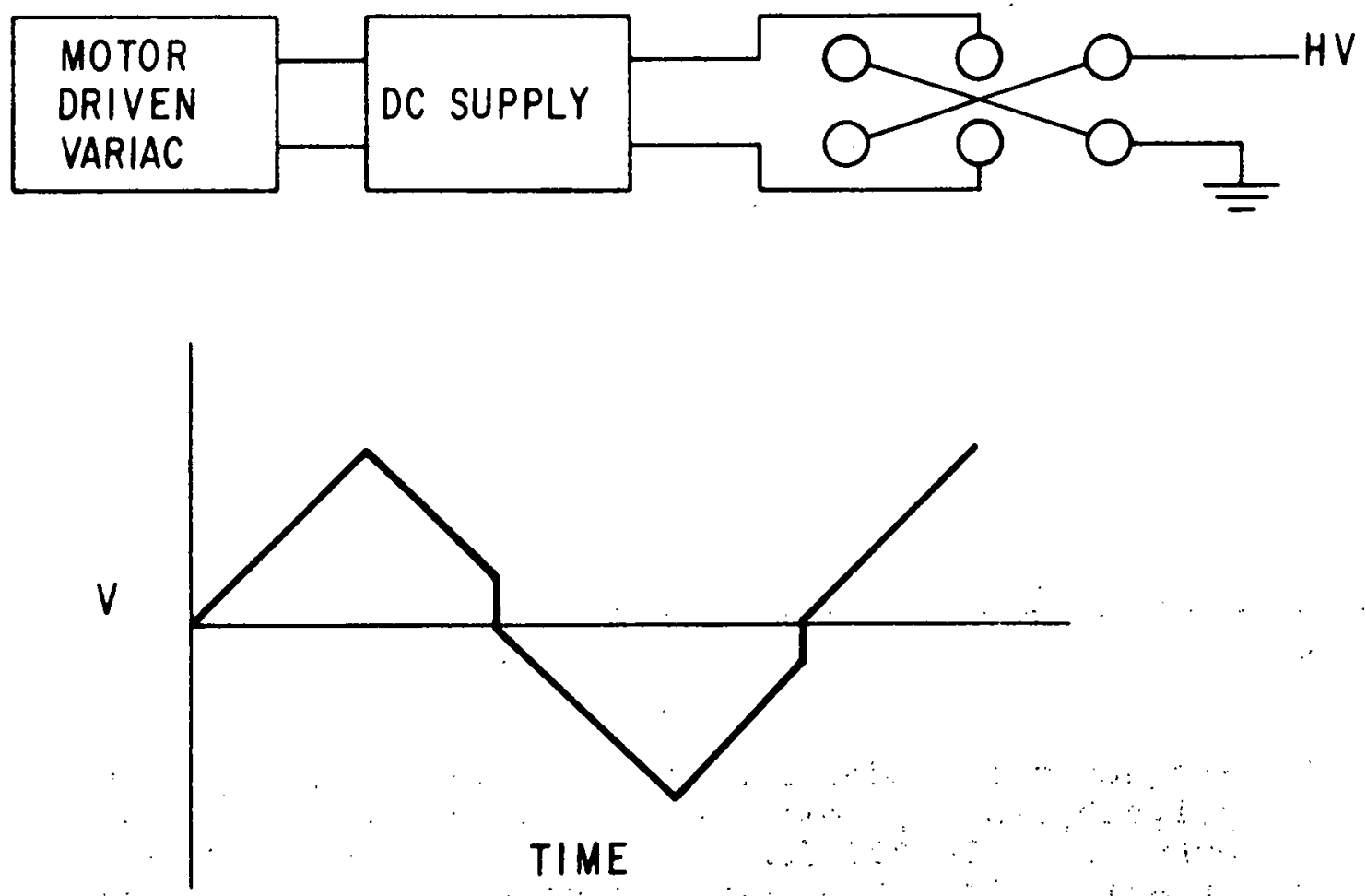

Fig. 36. Schematic of the system generating a low frequency saw tooth wave represented on the bottom of the figure. 
The results of these measurements are shown in Fig. 37. The voltages here refer to peak values. We have included points for pure polyethylene $(\mathrm{PE})$ at $60 \mathrm{~Hz}$ and with $\mathrm{DC}$ and for polyethylene containing $5 \%$-bromostearic acid (PEBS) at $60 \mathrm{~Hz}$. In order to reduce the voltage required these experiments utilize etched needles of $0.05 \mathrm{mil}$ radius and gaps of $30 \mathrm{mils}$. Except at $60 \mathrm{~Hz}$ with $\mathrm{PE}$ no trees were observed, so that the remaining points represent breakdown of the samples, or for those points

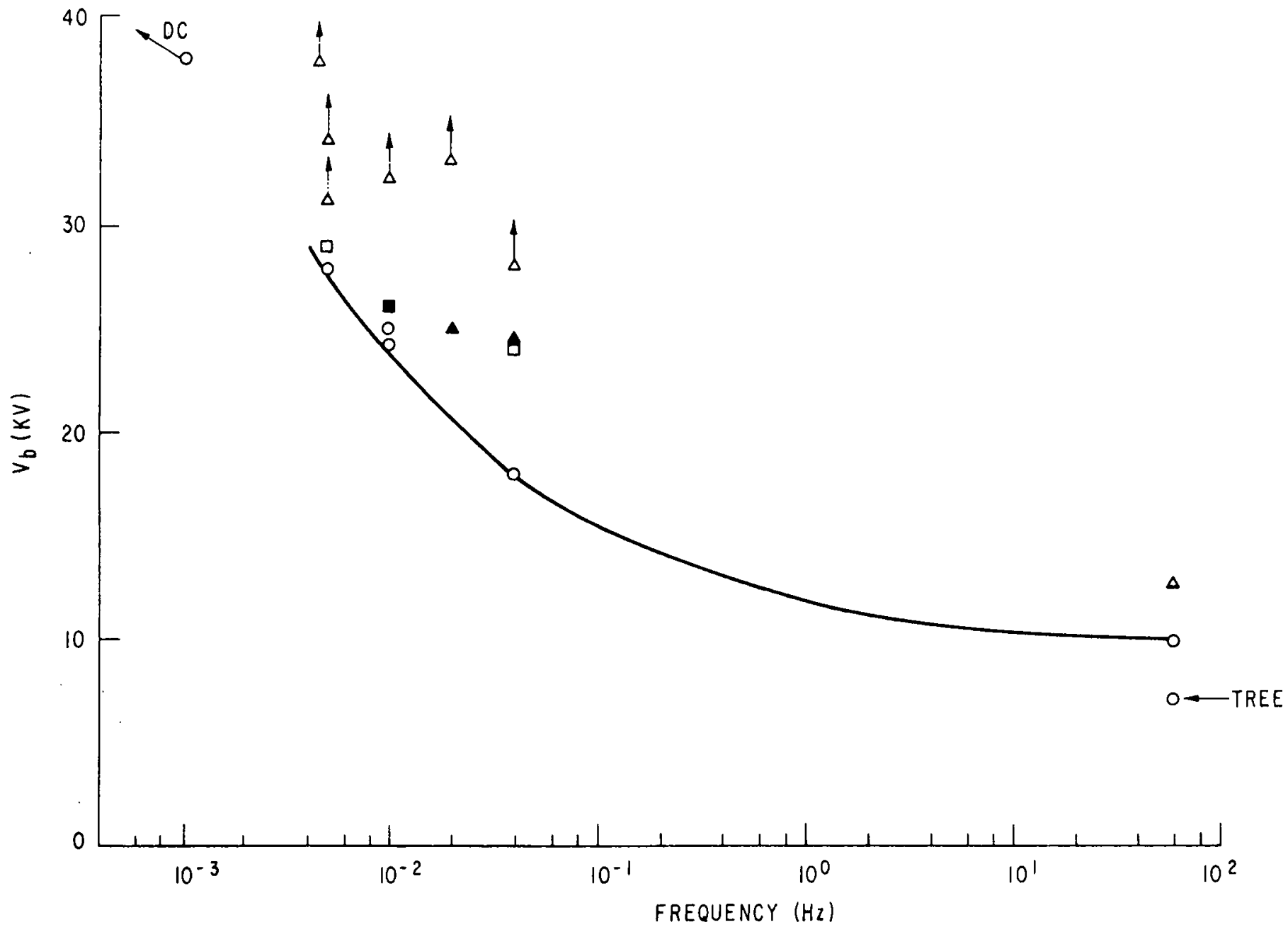

Fig. 37 Frequency dependence of the breakdown voltages of polyethylene systems: o pure polyethylene, $\square$ polyethylene $+5 \%$ L 25352E, $\Delta$ polyethylene $+5 \%$-bromostearic acid. Open symbols refer to breakdown on the positive wave while the solid symbols refer to breakdown on the negative half cycle. The arrows indicate samples where flashover occurred. 
shown with arrows; flashover in our system.: With lower frequencies it is possible to distinguish the polarity of the voltage..... wave on which breakdown occurs. The solid points refer to breakdown on the negative wave, while the open points refer, to the positive half cycle.

The increase in breakdown voltage with decreasing frequency strongly suggests that some field grading is occurring below 60 $\mathrm{Hz}$ even in pure PE.... This being true, it is to be expected that an increase in conductivity should further increase the breakdown voltage. This effect is clearly demonstrated in the PEBS system. It should be noted that if the mechanism of breakdown were purely thermal, increasing conductivity should produce a decrease in breakdown voltage. The addition of a Lubrizol, on the other hand, has only a marginal influence on breakdown. It will be recalled that the conductivity of the Lubrizol - PE system was markedly lower than that for PEBS.

The fact that in all cases with pure PE the breakdown occurred on the positive half cycle strongly suggests that. electrons are injected on the negative half cycle and trapped to produce an augmenting space charge field on the positive wave. This picture is consistent with the fact that when ionic addi-. tives are present the charge can dissipate sufficiently quickly so that breakdown may occur on either half cycle. That the entire effect of increasing breakdown voltage is not due to space charge dissipation is clear from the fact that the effect must be at most a factor of two while experimentally an increase of at least a factor of four is found. It. will be remembered that at $60 \mathrm{~Hz}$, with larger radii needles, the calculated maximum stress. for treeing was very nearly half of the so-called "intrinsic electric strength" (Part 1).

Using Eq. 4-1 of Part I, we calculate for the maximum field at the tip a.value of $1.4 \times 1.0^{7}$ volts $/ \mathrm{cm}$ for the tree inception voltage of $\mathrm{PE}$ at $60 \mathrm{~Hz}$. This is to be compared: with the value of $3 \times 10^{6}$ volts/cm for needles of radius ranging from 0.37 to 1.75 mils (Table 4-2, Part I). Whether or not field grading is 
of importance at $60 \mathrm{~Hz}$ in this system, it may be concluded that the maximum field for tree initiation increases with decreasing radius of the needle for needles of sufficiently small radii. This suggests that tree initiation may involve a critical field over a distance criterion such as would be the case for thermal or electron avalanche mechanisms.

It should be noted, however, that field grading in AC fields still requires that the Maxwell relaxation time be of the order of one quarter cycle. Otherwise capacitive rather than resistive field distributions would obtain.

Thus, the frequency dependence in polymers may be explained if the true volume conductivity is much lower than that measured. This could be true if, for example, the measured conductivity were determined by conducting paths. For $\alpha$-bromostearic acid the measured low field conductivity is about $10^{-15}$ mho $\mathrm{cm}^{-1}$ at $25^{\circ} \mathrm{C}$ so that the Maxwell relaxation time is about $200 \mathrm{sec}$, much longer than the $4 \times 10^{-3}$ sec time for a quarter cycle for $60 \mathrm{~Hz}$. However, it is the conductivity in the high field region which is of importance. Table 5.2 gives the enhancement factor for the conductivity as a function of field obtained from onsager. Using this table, the enhancement factor at $6 \times 10^{6}$ volt $\mathrm{cm}^{-1}$ ( $\mathrm{E}_{\mathrm{s}}$ for polyethylene) is approximately $10^{9}$, so that the relaxation time should be about $2 \times 10^{-7}$ seconds, much lower than that of a quarter cycle. Our measurements of volume conductivity, then, would need be in error by some four orders of magnitude in order to explain the fact that only a slight amount of grading appears to be occurring at $60 \mathrm{~Hz}$. Such an experimental error seems quite unlikely.

It should be noted that even if the conductivity is as low

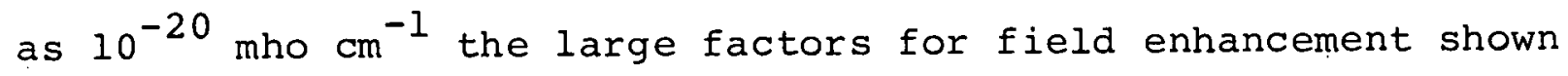
in Table 5-2 would permit limited field grading to occur. This presumably explains our earlier observations suggesting that some field grading occurred with pure polyethylene. 


\section{TABLE 5-2}

\section{Conductivity Enhancement Factor $(\sigma / \sigma 0)$}

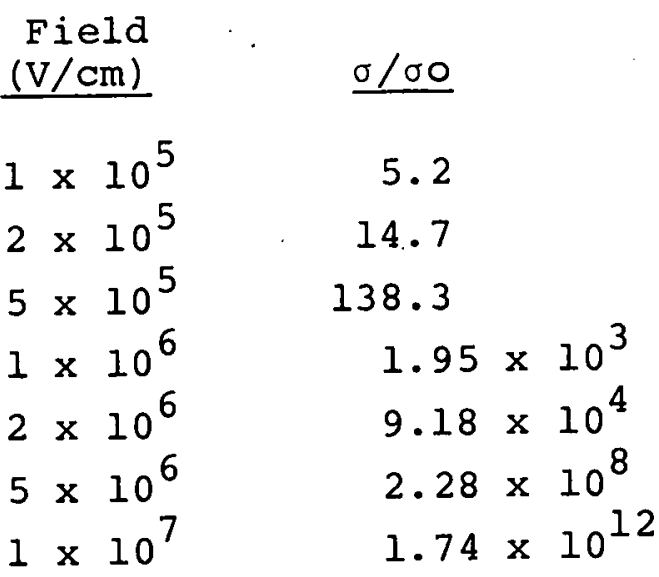

We are thus faced with the following dilemma. Our calculations show that according to the onsager theory the field dependence of conductivity should not change with frequency for sinusoidal voltages. However, our low frequency high voltage experiments do show an increase in breakdown voltage with decreasing frequency, suggesting that field grading is becoming more efficient. The fact that increasing the conductivity at low frequencies by adding $\alpha$-bromostearic acid raises the breakdown voltage appears to be very strong evidence for increased field grading at lower frequencies, since almost any other mechanism of breakdown would require no change or a decrease in breakdown voltage. Direct measurements of the variation in high field conductivity with frequency in polymeric systems therefore become crucial to a resolution of this dilemma. Also a re-examination of onsager's theory seems to be indicated.

These results may be summarized as follows:

1. Onsager's time dependent theory predicts no dependence of ionic high field conductivity on frequency.

2. Experimentally this has been confirmed for liquid hydrocarbon solutions.

3. Breakdown voltages in point to plane geometries increase with decreasing frequency below $60 \mathrm{~Hz}$ in PE, PEBS and PE-L25352E. 
4. At low frequencies the breakdown voltage for PE increased on addition of $a$-bromostearic acid.

5. For very sharp points ( $\sim .05 \mathrm{mil}$ radius) the maximum field at the point, assuming no field grading, greatly exceeds the "intrinsic" electric strength of PE, particularly at low frequencies.

6. There is a polarity effect which depends upon the nature of the additive. 


\section{REFERENCES}

1. L. Onsager, J. Chem. Phys. 2, 605 (1934)

2. M. Abramowitz and I: Stegun, Handbook of Mathematical Functions, Dover Publications, Inc., New York, 1970, p. 260.

3. P. Fischer, NRC-NAS Conference on Electrical Insulation and Dielectric Phenomena, 1974, p. 661.

4. Technical Proposal to EPRI, CR\&D 3351.008E, 1973,. p. 9-5.

5. R. Fuoss and F. Accascina, Electrolytic Conductance, Interscience Publishers, Inc., New York, 1959, Chapter 17.

6. S. A. Birnbaum, Shou-Cheng, J. Fu and T. P. Greenstein, J.: Bio. Chem. 203, 333 (1953).

7. R. E. Barker and C. R. Thomas, J. Appl. Phy. 35, 90 (1964).

8. S. Fujiki, H. Furisawa, T. Kuhara and H. Matsuba, IEEE Trans. Paper no. 71TP195-PWR, February 1971.

9. H. Kato, N. Maekawa, S. Inoue, and H. Fujita, NRC-NAS Conference on Electrical Insulation and Dielectric Phenomena, 1974 , p. 229.

10. A. O. Allen and A. Hummel, Disc. Far. Soc. 36, 95 (1963). 
Appendix. 1

Field Calculation for Concentric sphere Geometry

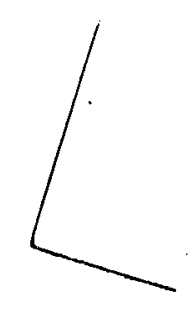

Starting with:

$$
\begin{aligned}
\nabla \cdot j & =0 \\
j & =\sigma E
\end{aligned}
$$

With the spherical symmetry shown in Fig. 1 the divergence operator, produces for Eq. $(7-1)$.

i.e.,

$$
\begin{aligned}
& \frac{1}{r^{2}} \frac{d}{d r}\left(r^{2} j\right)=0 \\
& 2 r j+\frac{r^{2} d j}{d r}=0
\end{aligned}
$$

Substituting $(7-2)$ into $(7-4)$ gives

$$
2 r_{\sigma} E+r^{2}\left[E \frac{d \sigma}{d r}+\sigma \frac{d E}{d r}\right]=0
$$

which may be written

$$
2 \sigma E+r\left[E \frac{d \sigma}{d E} \cdot \frac{d E}{d r}+\sigma \frac{d E}{d r}\right]=0
$$

or

$$
2 E+r \frac{d E}{d r}\left[E \frac{d l n \sigma}{d E}+1\right]=0
$$

This may be separated to give

$$
\left[E \frac{d l n \sigma}{d E}+1\right] \quad \frac{d E}{E}=-2 \frac{d r}{r}
$$

and, on integration

$$
\ln \sigma+\ln E=-2 \ln r+\ln B
$$

or

$$
\frac{B}{r^{2}}=\sigma E
$$


where $\mathrm{B}$ is an integration constant. This is the general solution for the field as a function of $r$, valid for any dependence of $\sigma$ on $\mathrm{E}$.

If for example, $\sigma$ is a constant, $\sigma_{o^{\prime}}$ i.e., the material is Ohmic, then Eq. (7-10) gives

$$
E=\frac{B}{\sigma_{O} r^{2}}
$$

and the field decreases as the inverse square of the distance from the center of the sphere.

If field-enhanced ionic dissociation occurs, then for low dielectric constant materials, onsager's ${ }^{(1)}$ treatment gives

$$
\sigma=\sigma_{0}[F(b)]^{1 / 2}
$$

where

$$
\mathrm{b}=\frac{9.636 \mathrm{E}}{\varepsilon \mathrm{T}^{2}}
$$

$T$ is the absolute temperature and $\varepsilon$ is the dielectric constant. For large fields

$$
[F(b)]^{1 / 2} \rightarrow\left(\frac{2}{\pi}\right)^{1 / 4}(8 b)^{-3 / 8} e^{(2 b)^{1 / 2}}
$$

For $E>2 \times 10^{5}$ volt $/ \mathrm{cm}$, the error in (7-13) and thus in $\sigma$ is less than $2 \%$ for polyethylene $\left(\varepsilon=2.2\right.$ and $\left.T=298^{\circ} \mathrm{K}\right)$. Equations (7-12) and (7-13) may be rewritten

$$
\sigma=A_{\sigma} E^{-3 / 8} e^{\alpha E^{1 / 2}}
$$

where $A=0.175\left(\varepsilon \mathrm{T}^{2}\right)^{3 / 8}\left(\text { volt } \mathrm{cm}^{-1}\right)^{3 / 8}$

and $\alpha=\frac{4.39}{\left(\varepsilon \mathrm{T}^{2}\right)^{1 / 2}}$ (volt $\left.\mathrm{cm}^{-1}\right)^{-1 / 2}$

Substituting $\sigma$ from $(7-14)$ into $(7-10)$ gives for $E$

$$
E=\left[\frac{B}{A \sigma_{0}} \cdot \frac{1}{r^{2}} \cdot e^{-\alpha E^{1 / 2}}\right]^{8 / 5}
$$


Unfortunately, this equation is transcendental, and must be solved either graphically or by an iterative procedure once $B$ is known. It clearly shows, however, the grading effect expected, since the $\left(1 / \mathrm{r}^{2}\right)$ dependence found in Eq. (7-11) for ohmic materials is tempered by the highly field-sensitive damping term $e^{-\alpha E^{l / 2}}$.

It remains to determine the integration constant, B, using the relation

$$
V=\int_{r_{0}}^{R} \operatorname{Edr}
$$

where $\mathrm{V}$ is the applied potential.

Differentiating our general solution, (7-10)

$$
\mathrm{dr}=\frac{-\mathrm{B}^{1 / 2}}{2}(\sigma E)^{-3 / 2}\left[\sigma \mathrm{dE}+E \mathrm{~d}_{\sigma}\right]
$$

and substituting $(7-17)$ in $(7-16)$

$$
V=\frac{-B^{1 / 2}}{2}\left[\int_{E\left(r_{0}\right)}^{E(R)} \sigma^{-1 / 2} E^{-1 / 2} d E+\int_{\sigma\left(r_{0}\right)}^{\sigma(R)} \sigma^{-3 / 2} E^{1 / 2} d \sigma\right](7-18)
$$

If we now assume that $r_{0}<<R$, as will usually be true for cases of interest to us, then the upper limits on the integrals in $(7-18)$ may be replaced for practical purposes by

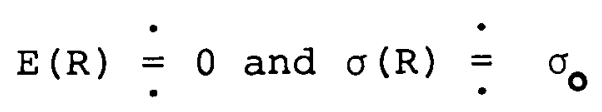

Once having specified a relationship between $\sigma$ and $\mathrm{E}$, (7-18) can be written

$$
B=\frac{4 V^{2}}{f^{2}\left[E\left(r_{0}\right)\right]}
$$

where 
$f\left[E\left(r_{0}\right)\right]=\int_{E\left(r_{0}\right)}^{0} \sigma^{-1 / 2} E^{-1 / 2} d E+\int_{\sigma\left(r_{0}\right)}^{\sigma_{0}} \sigma^{-3 / 2} E^{1 / 2} d \sigma \quad(7-21)$

The complete solution is then given, by combining $(7-20)$ with $(7-10)$, as

$$
E=\frac{4 V^{2}}{f^{2}\left[E\left(r_{0}\right)\right] \sigma r^{2}}
$$

The solution is general, that is, it is valid for any assumed functional relationship between $\sigma$ and $E$. Let us examine two cases of interest.

Case 1: Ohmic

$$
\begin{array}{r}
\text { If } \sigma=\sigma_{0}=\text { const. }, \text { then from }(7-21) \\
\quad f\left[E\left(r_{0}\right)\right]=\frac{-2\left[E\left(r_{0}\right)\right]^{1 / 2}}{\sigma_{0}^{1 / 2}}
\end{array}
$$

since the second integral in $(7-21)$ is zero. Substituting in $(7-22)$ gives

$$
E=\frac{v^{2}}{E\left(r_{0}\right) r^{2}}
$$

Evaluating this at $r_{0}$ and substituting back for $E\left(r_{0}\right)$ gives

$$
E=\frac{V r_{0}}{r^{2}}
$$

which is the well known expression for this case.

\section{Case 2: Onsager}

With a field-dependent conductivity given by $(7-14),(7-21)$ gives 


$$
\begin{gathered}
f\left[E\left(r_{0}\right)\right]=\int_{E\left(r_{0}\right)}^{0} A^{-1 / 2} \sigma_{0}^{-1 / 2} E^{-5 / 16} e^{\frac{-\alpha E^{1 / 2}}{2}} d E \\
-2 \int_{\sigma\left(r_{0}\right)}^{\sigma} E^{1 / 2} d \sigma^{-1 / 2}
\end{gathered}
$$

since $\sigma^{-3 / 2} d \sigma=-2 d \sigma^{-1 / 2}$

Further substitution for $\mathrm{d}^{-1 / 2}$ from (7-14) produces

$$
\begin{gathered}
f\left[E\left(r_{0}\right)\right]=A^{-1 / 2} \sigma_{0}^{-1 / 2}\left\{\frac{5}{8} \int_{E\left(r_{0}\right)}^{0} E^{-5 / 16} e^{\frac{-\alpha E^{1 / 2}}{2}} d E\right. \\
\left.+\frac{\alpha}{2} \int_{E\left(r_{0}\right)}^{0} E^{3 / 16} e^{\frac{-\alpha E^{1 / 2}}{2}} d E\right\}
\end{gathered}
$$

The integrals in (7-27) are simply related to incomplete gamma functions ${ }^{(2)}$ and can be evaluated using a routine available in our computer library. Writing $2 Y$ for the quantity in brackets in $(7-27)$ we have

$$
\mathrm{f}\left[\mathrm{E}\left(\mathrm{r}_{0}\right)\right]=2 \mathrm{YA}^{-1 / 2} \sigma_{0}^{-1 / 2}
$$

The function $\mathrm{Y}$ is plotted in $\mathrm{Fig} \cdot 7-1$ against $\mathrm{E}\left(\mathrm{r}_{0}\right)$. The field, then, is given from $(7-22),(7-28)$ and $(7-14)$ as

$$
E(r)=\left[\frac{v^{2}\left\{e^{\left.-\alpha[E(r)]^{1 / 2}\right\}}\right.}{Y^{2} r^{2}}\right]^{8 / 5}
$$




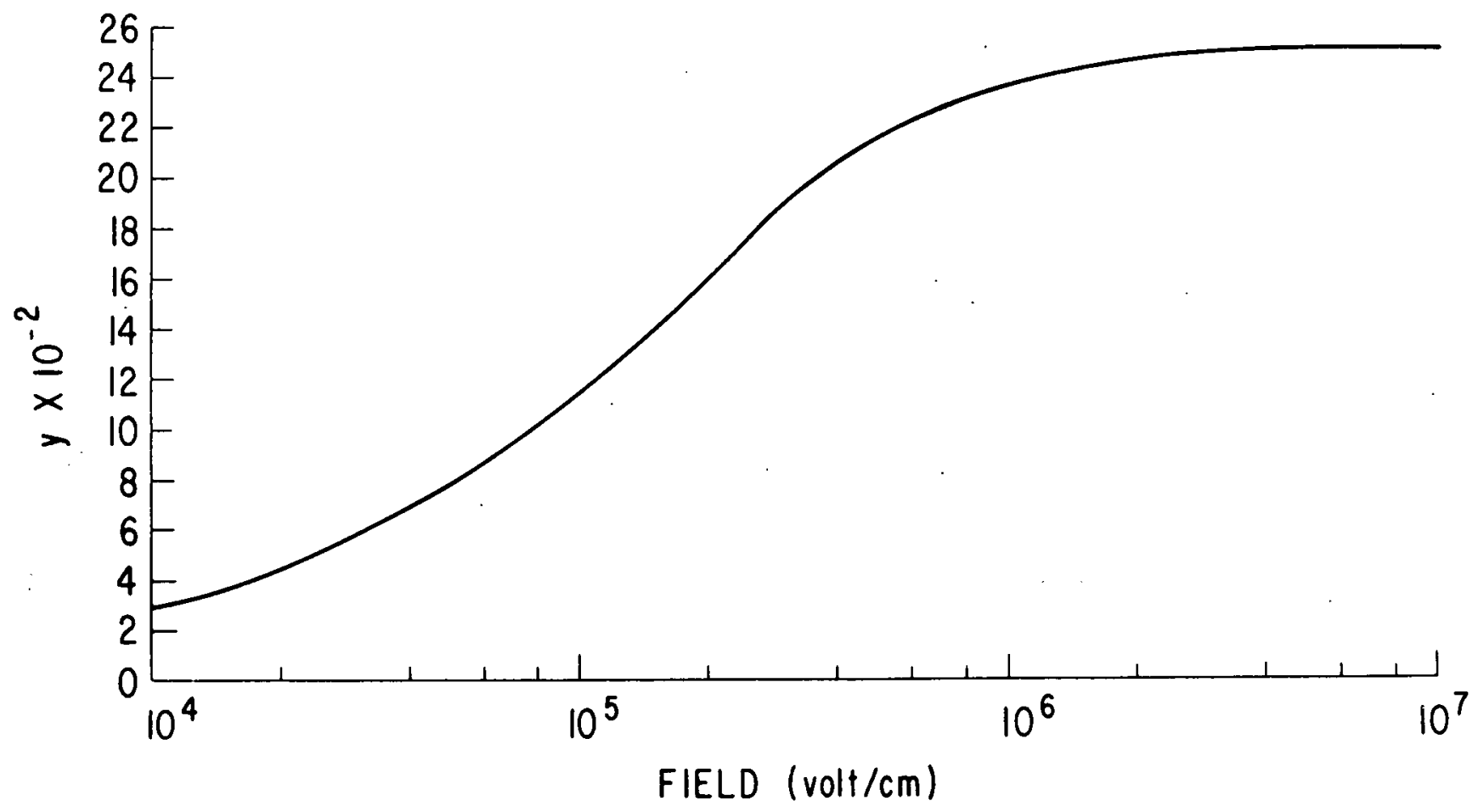

Fig. 7-1 The dependence of the function $Y$ on field. cf. Eq. $(7-27)$ and $(7-28)$.

This transcendental equation can be solved by first choosing a value for $\mathrm{E}\left(\mathrm{r}_{0}\right)$, then calculating the corresponding voltage, $V$, from (7-29) for $r=r_{0}$, and using an iterative or graphic procedure to evaluate $E$ as a function of $r$, for these values of $V$ and $E\left(r_{0}\right)$. The procedure may then be repeated for different values of $\mathrm{E}\left(\mathrm{r}_{\mathrm{O}}\right)$.

In our work we are primarily interested in values of the field at the point so that we shall not presently carry out the calculations described above. If we assume that treeing initiates when the field at the point equals the intrinsic strength of the material, $E_{S}$, then Eq. (7-29) predicts for the treeing inception voltage.

$$
v_{t}=E_{s} 5 / 16 . Y_{0} e^{\frac{\alpha E_{s}}{2}}
$$

Since in general the intrinsic strengths of materials of interest are greater than $10^{6} \mathrm{~V} / \mathrm{cm}$ (polyethylene is about $7 \times 10^{6} \mathrm{~V} / \mathrm{cm}$ ) 
Fig. 7-1 indicates that $Y$ will be essentially a constant in this equation.

Appendix 2

Dielectric measurements

Loss tangent as a function of temperature and frequency and DC resistance as a function of temperature and voltage are presented in this section. These results are summarized in the text in Tables $4-5,4-6,4-9$ and $4-10$.

The following set of symbols are used uniformly throughout:

$$
\begin{aligned}
& \nabla-25^{\circ} \mathrm{C} \\
\tan \delta \quad & 055^{\circ} \mathrm{C} \\
& \Delta-65^{\circ} \mathrm{C} \\
& 0-85^{\circ} \mathrm{C} \\
& \nabla-50 \text { volt } \\
\text { resistance } \quad & 0-100 \text { volt } \\
& \Delta-500 \text { volt } \\
& \square-1000 \text { volt }
\end{aligned}
$$

Where the symbols are accompanied by upward arrows, the resistance is greater than $10^{15} \mathrm{ohms}$, the limit of our measurements. Except when otherwise specified, the samples are $100 \mathrm{mil}$. in thickness. Complete data on other thicknesses are not included here.

It will be realized that the slopes of the Arrhenius plots, and thus the activation energies, will be the same for resistivity as for resistance (cf Eq. 4-2), although the intercepts will be different. In view of the greater reliability of the high temperature data, greater weight has been given to these in drawing the lines. 


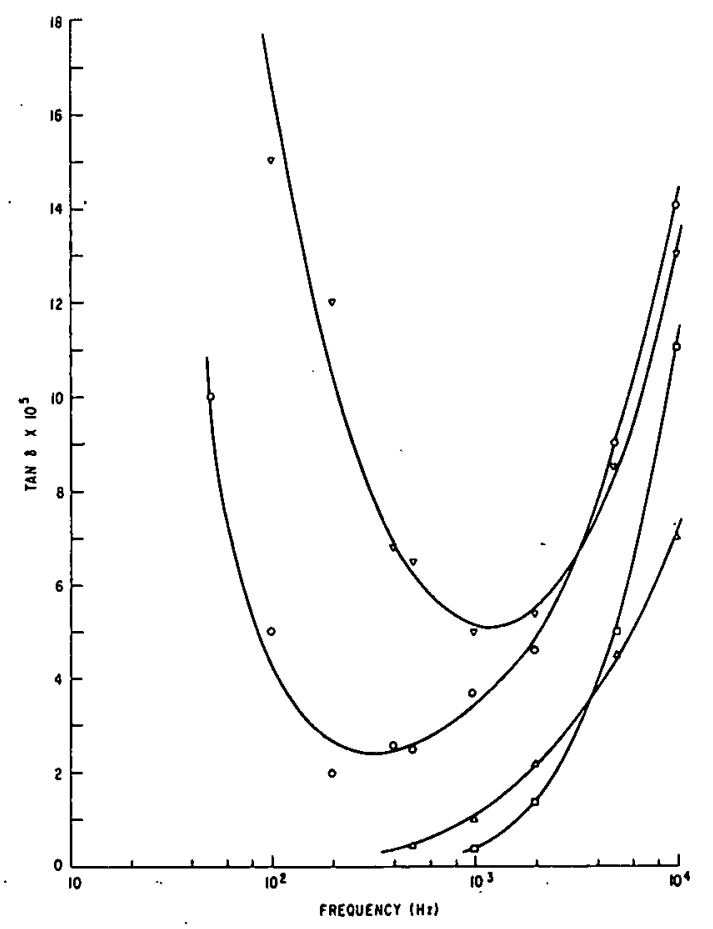

Fig. 7-2. Frequency dependence of the loss tangent. Pure polyethylene.

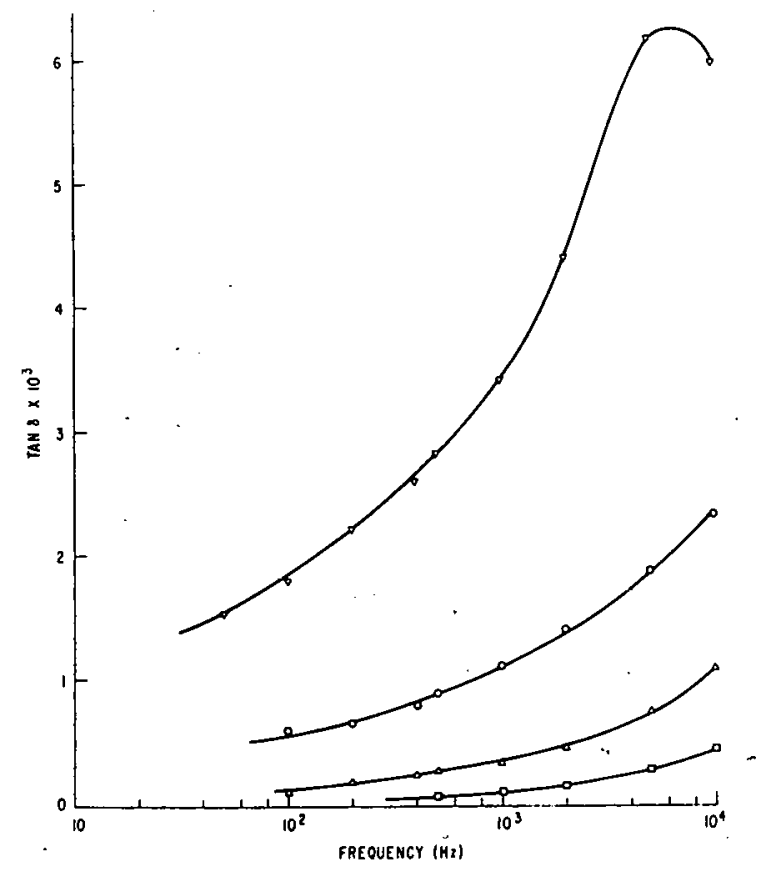

Fig. 7-3 Frequency dependence of the loss tangent. Pure vinyl acetate-ethylene copolymer. 


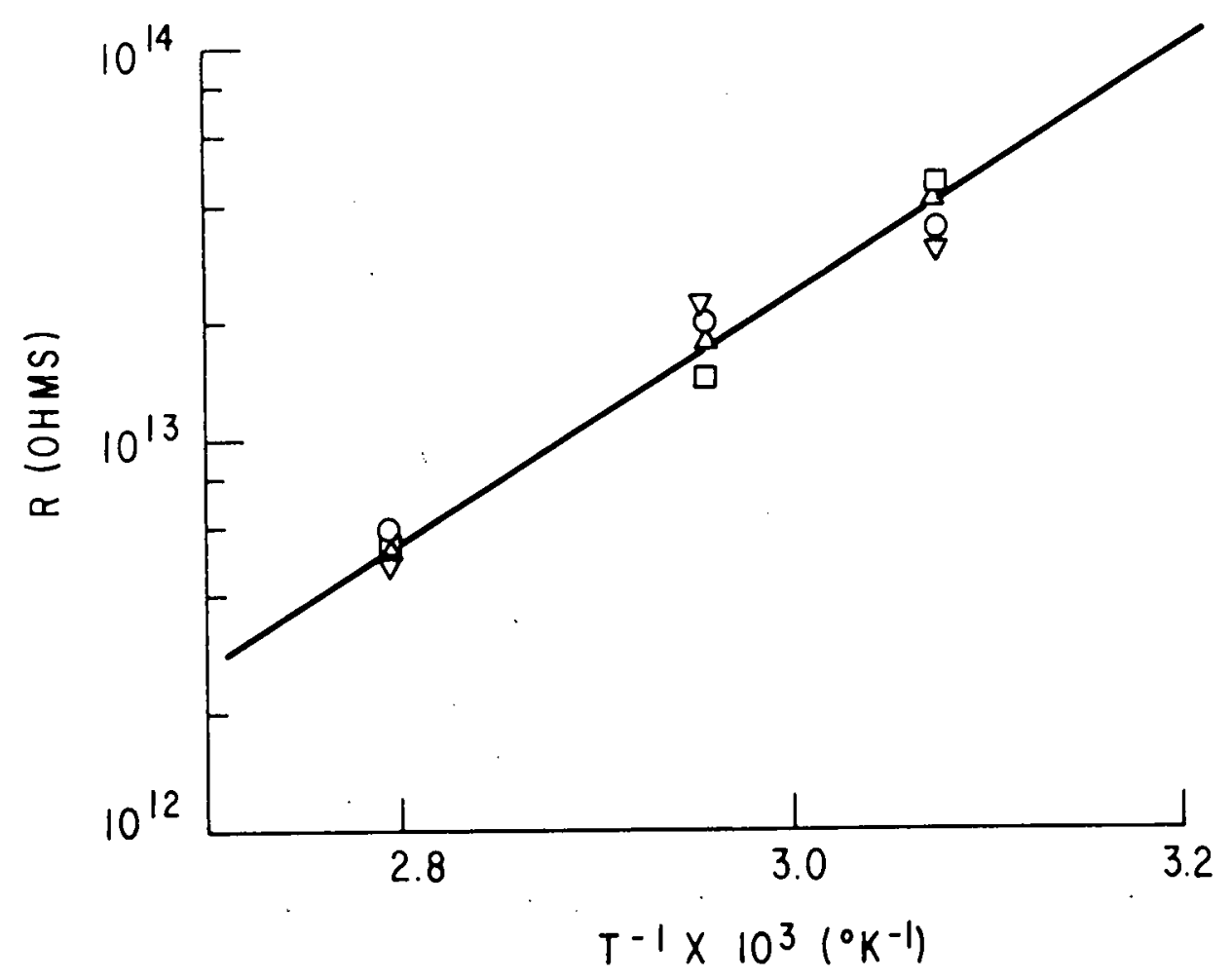

Fig. 7-4 Arrhenius plot. Pure vinyl acetateethylene copolymer.

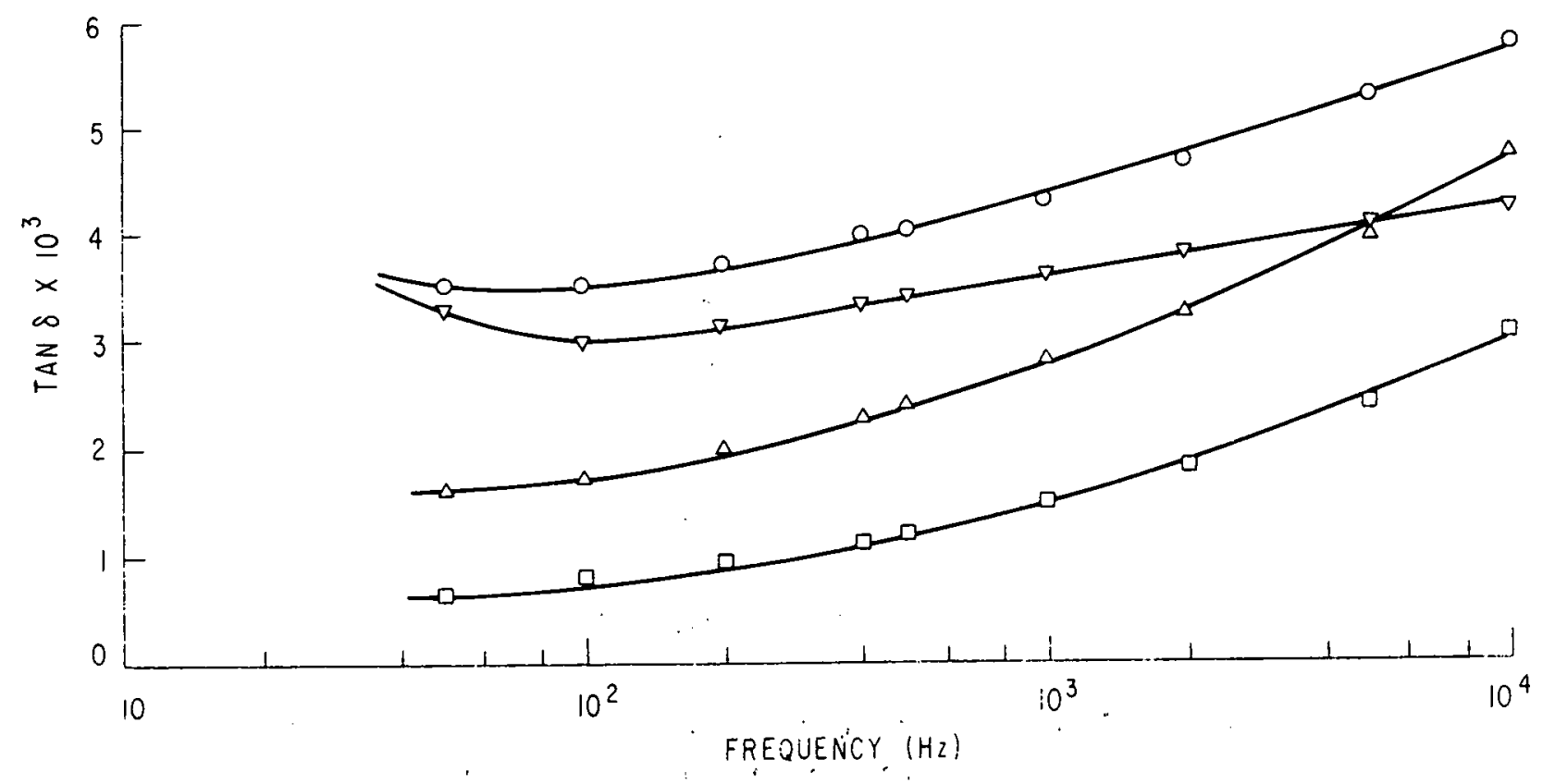

Fig. 7-5 Frequency dependence of the loss tangent. Ionomer. 


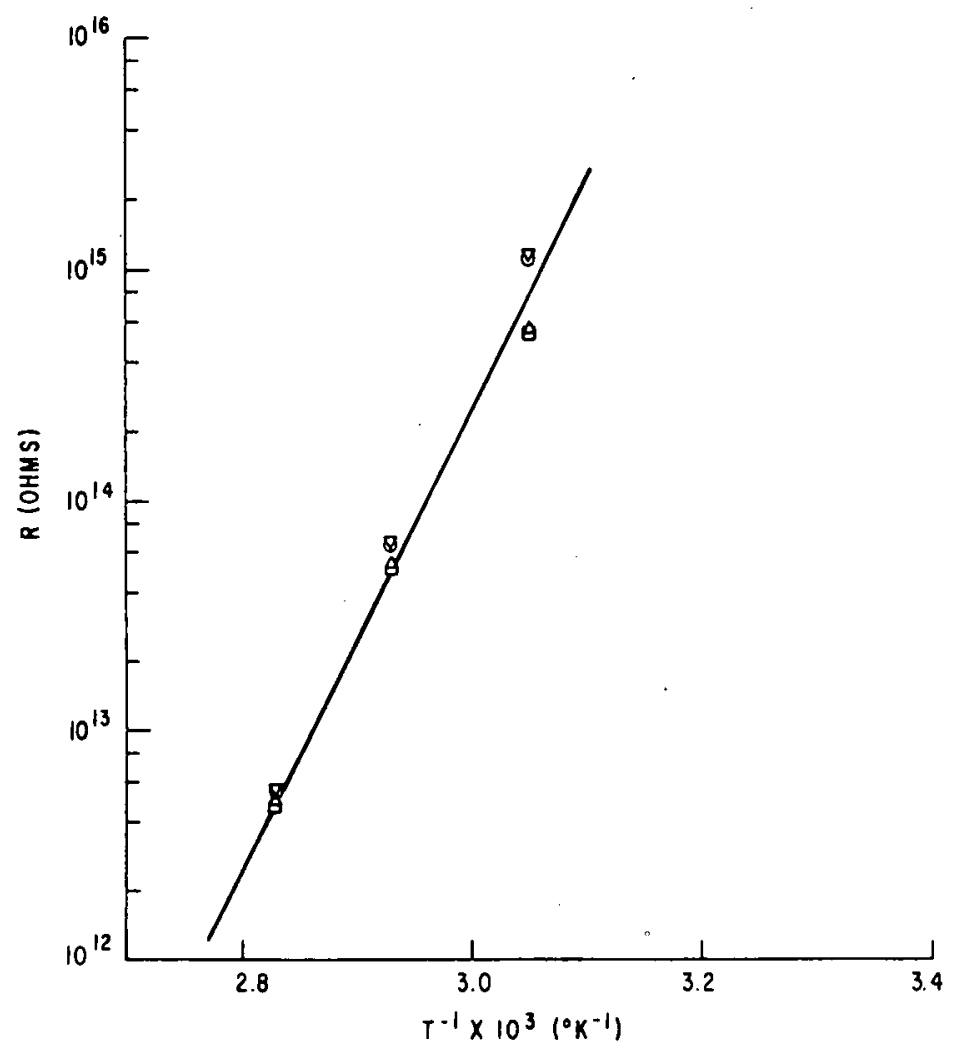

Fig. 7-6 Arrhenius plot. Ionomer.

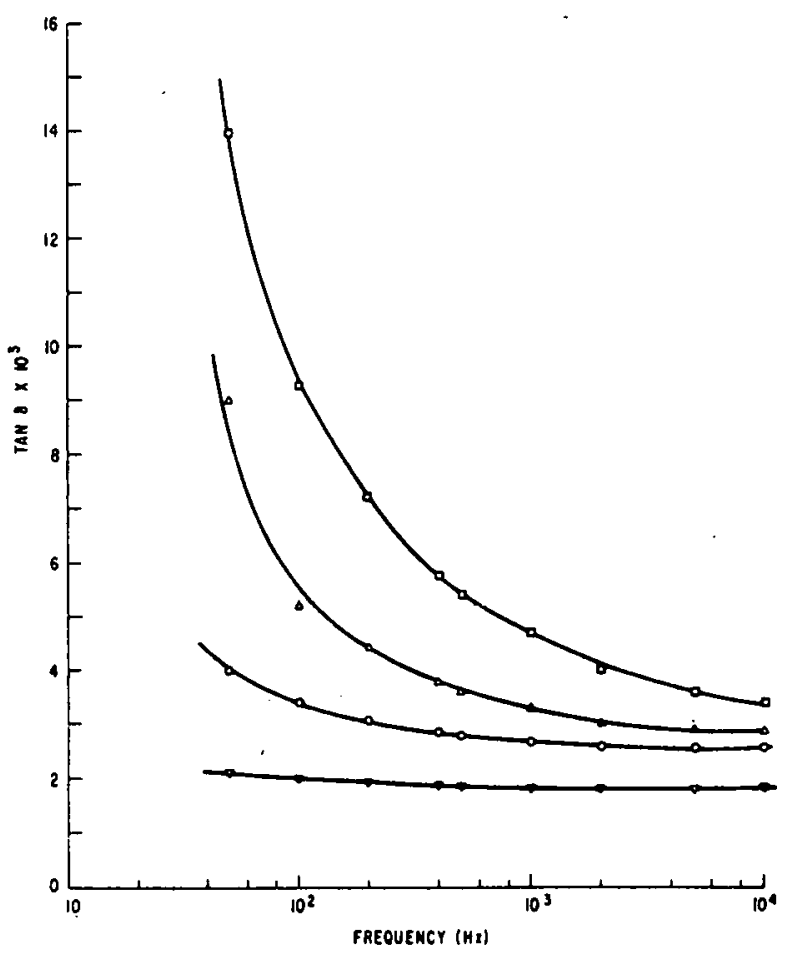

Fig. 7-7 Frequency dependence of the loss tangent. $\mathrm{PE}+4 \% \mathrm{AD} 48$. 


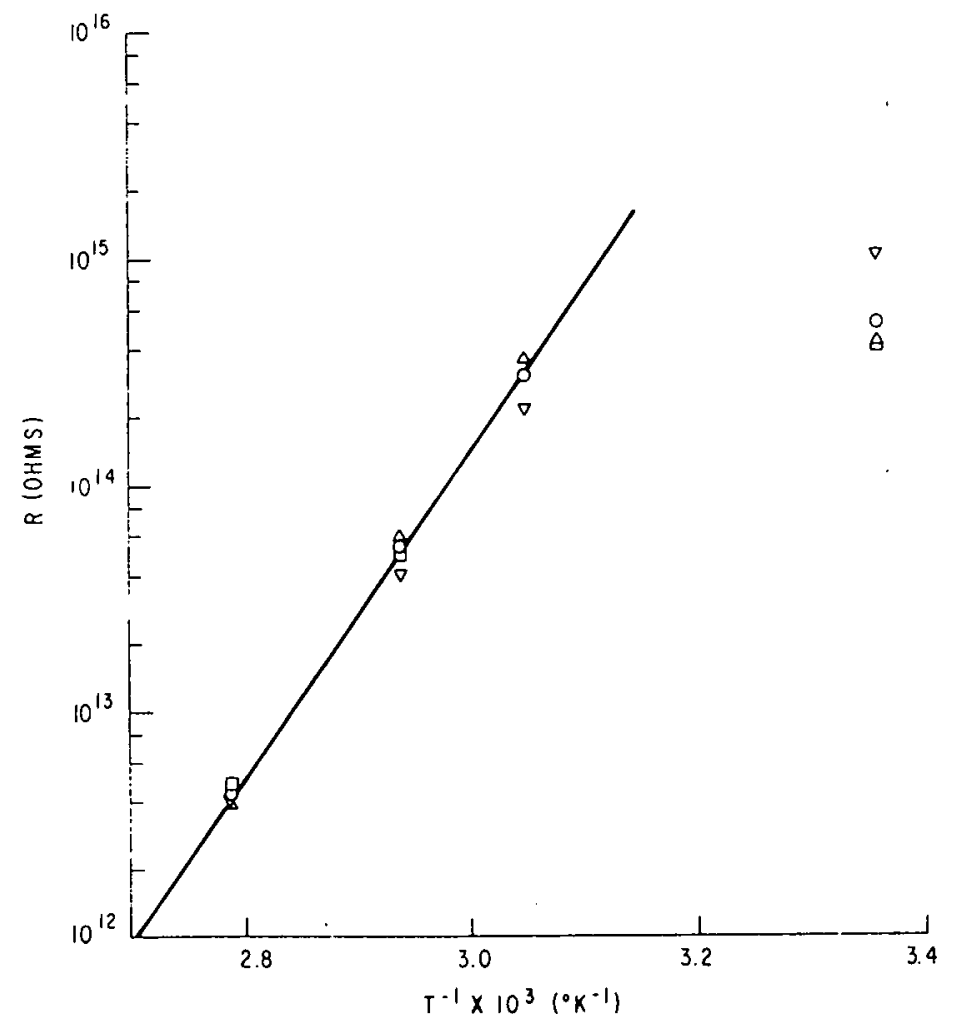

Fig. 7-8" Arrhenius plot. PE + 4\% AD48.

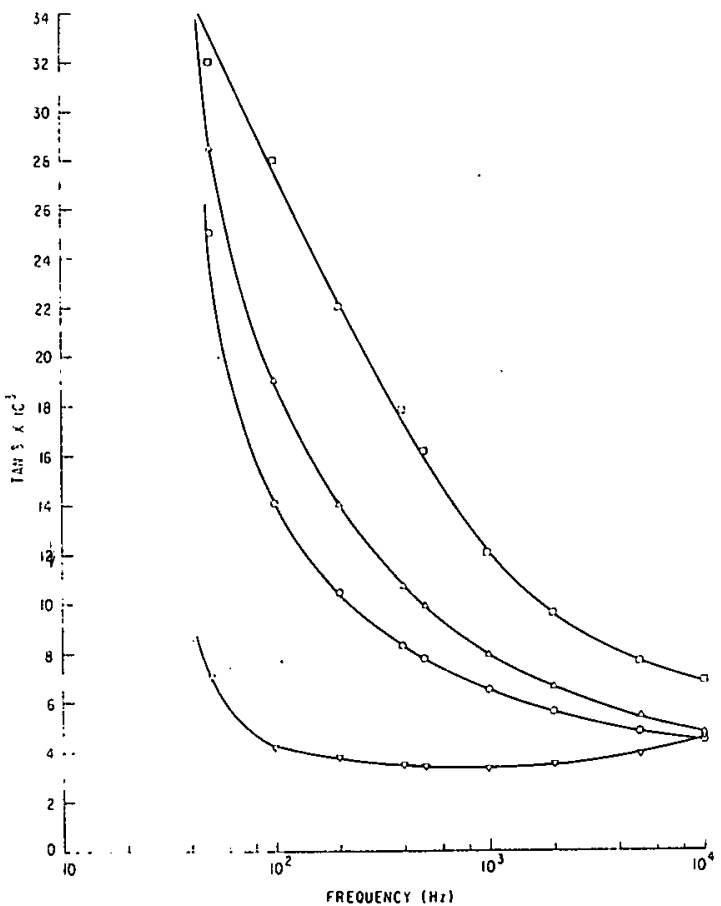

Fig. 7-9 Frequency dependence of loss tangent. $\mathrm{PE}+4 \% \mathrm{AD} 48+7 \%$ Pyranol 1499. 


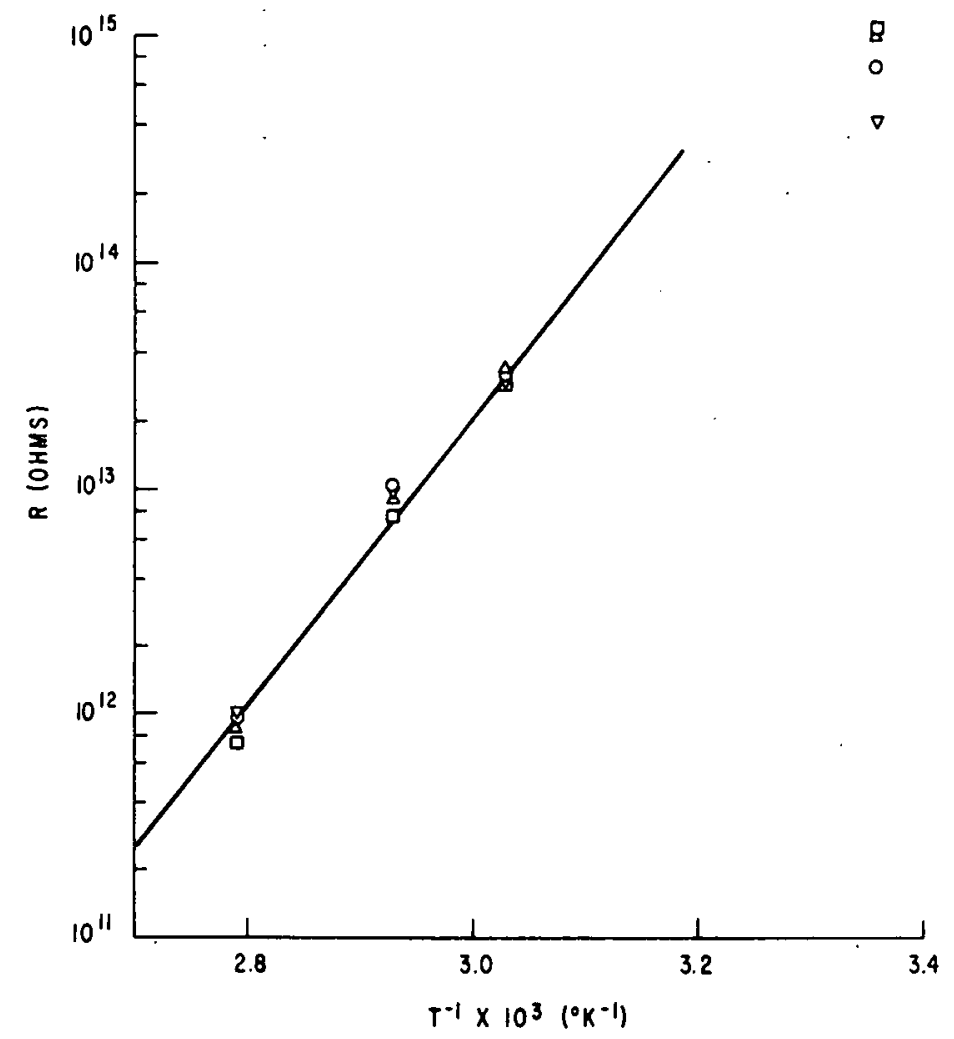

Fig. 7-10 Arrhenius plot. PE + 4\% AD48+7\% Pyranol 1499.

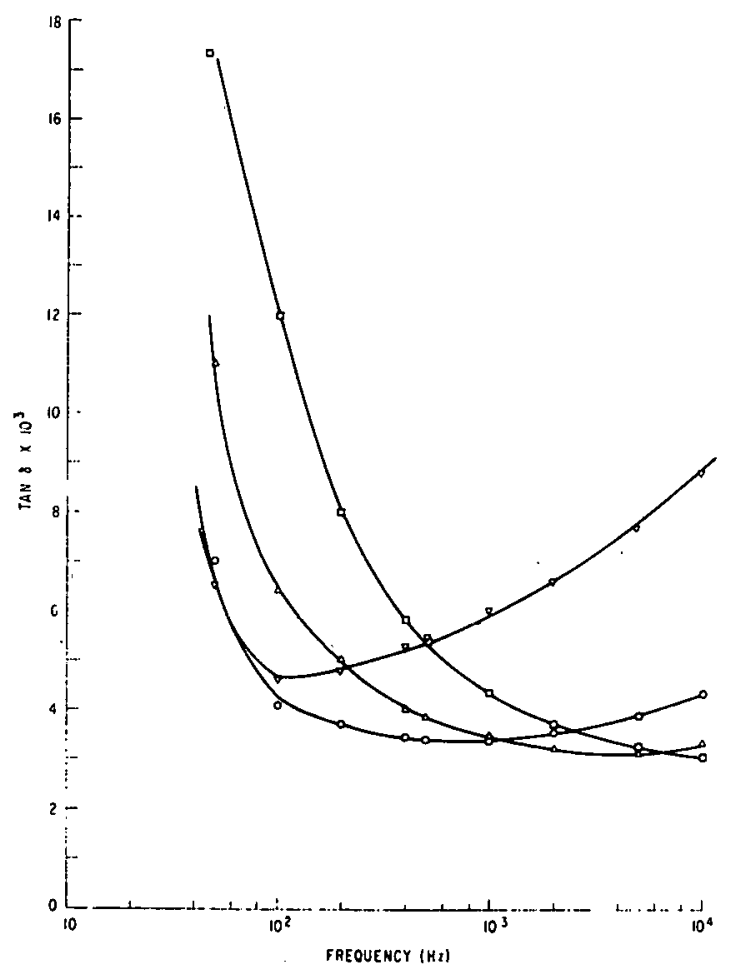

Fig. 7-11 Frequency dependence of loss tangent. PVAP $+4 \%$ AD 48 . 


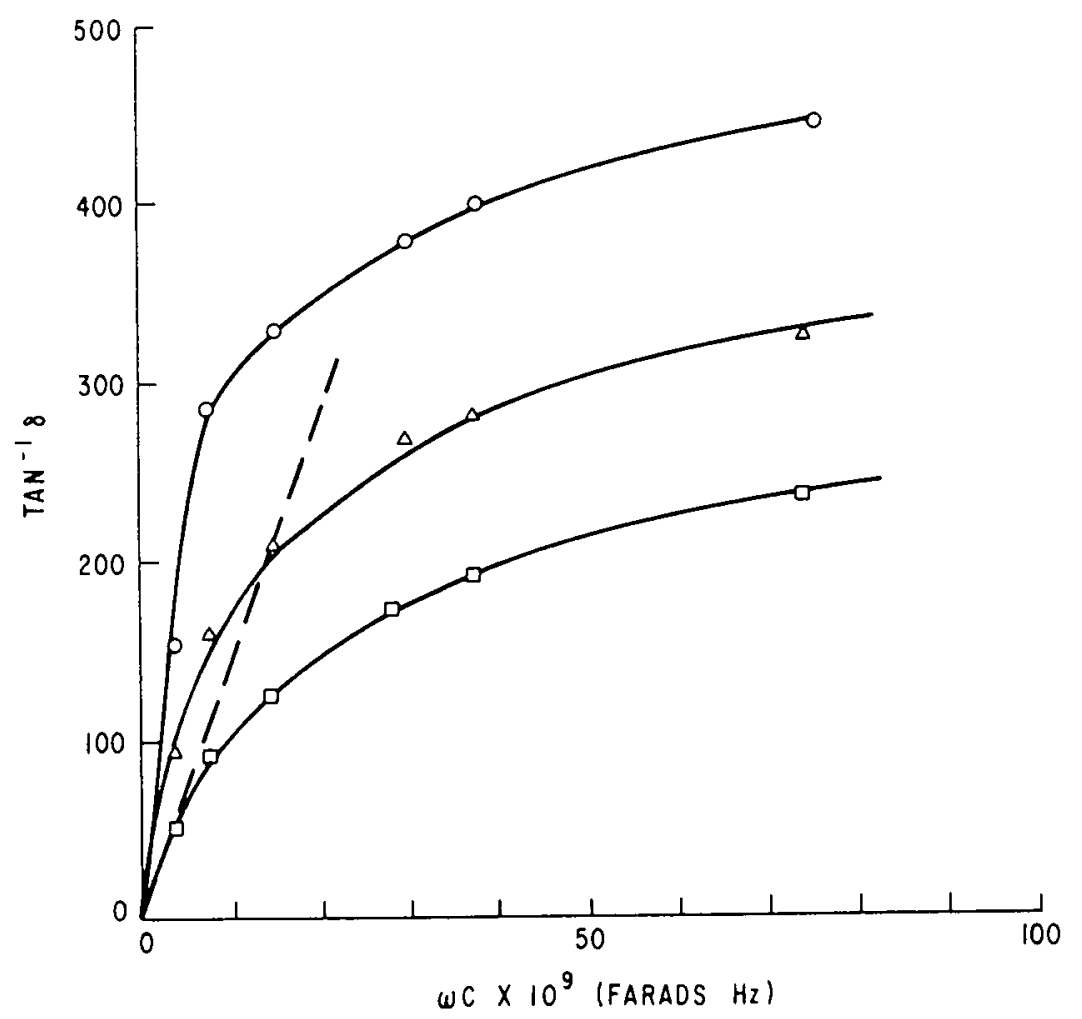

Fig. 7-12 Frequency dependence of reciprocal loss tangent PVAP $+4 \%$ AD 48 .

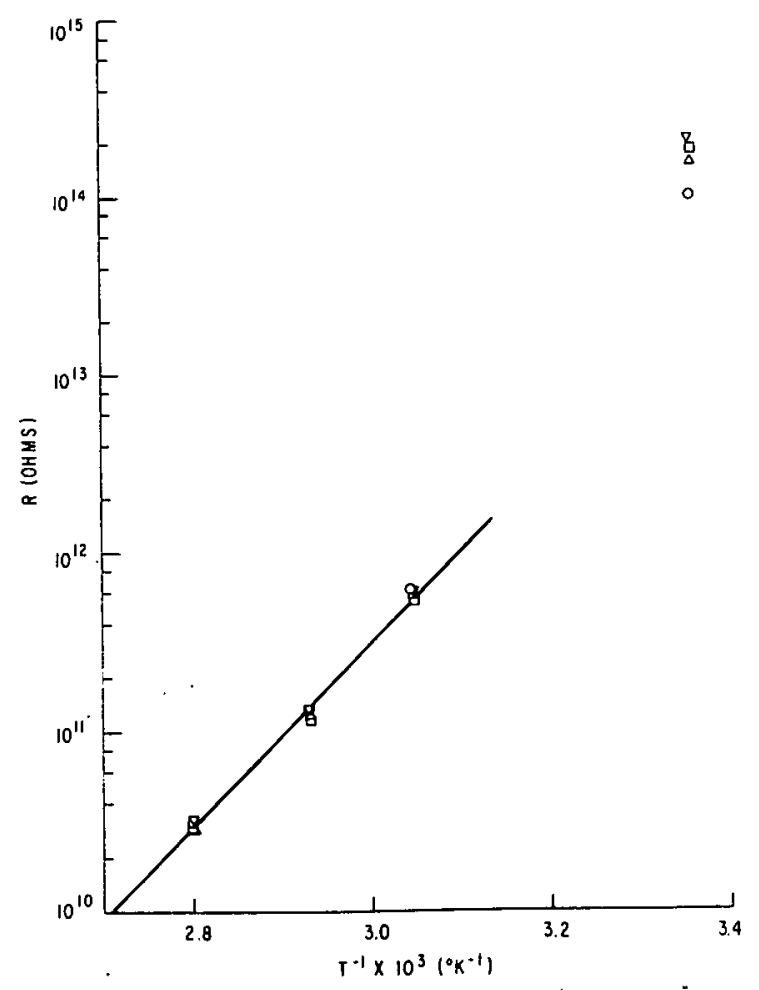

Fig. 7-13 Arrhenius Plot. PVAP + 4\% AD48. 


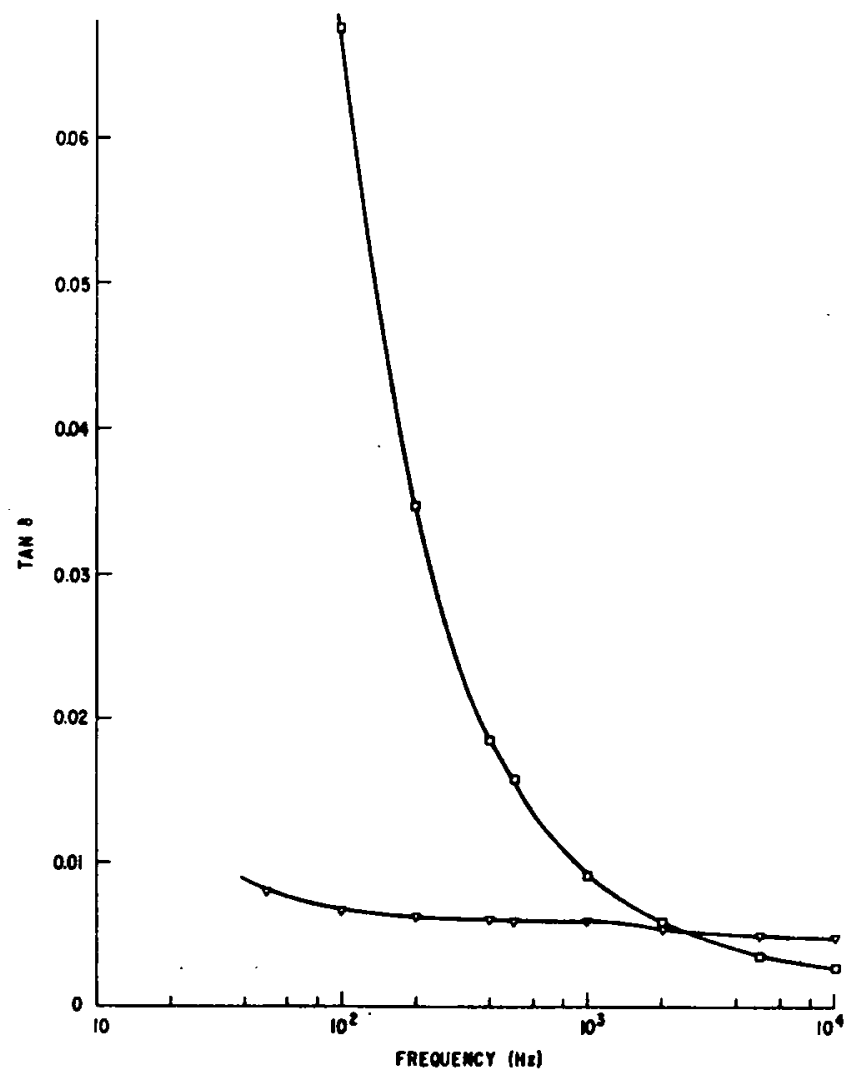

Fig. 7-14 Frequency dependence of loss tangent PVAP + 4\% AD $48+58 \%$ Pyranol 1499. Thickness $3 \mathrm{mil}$.

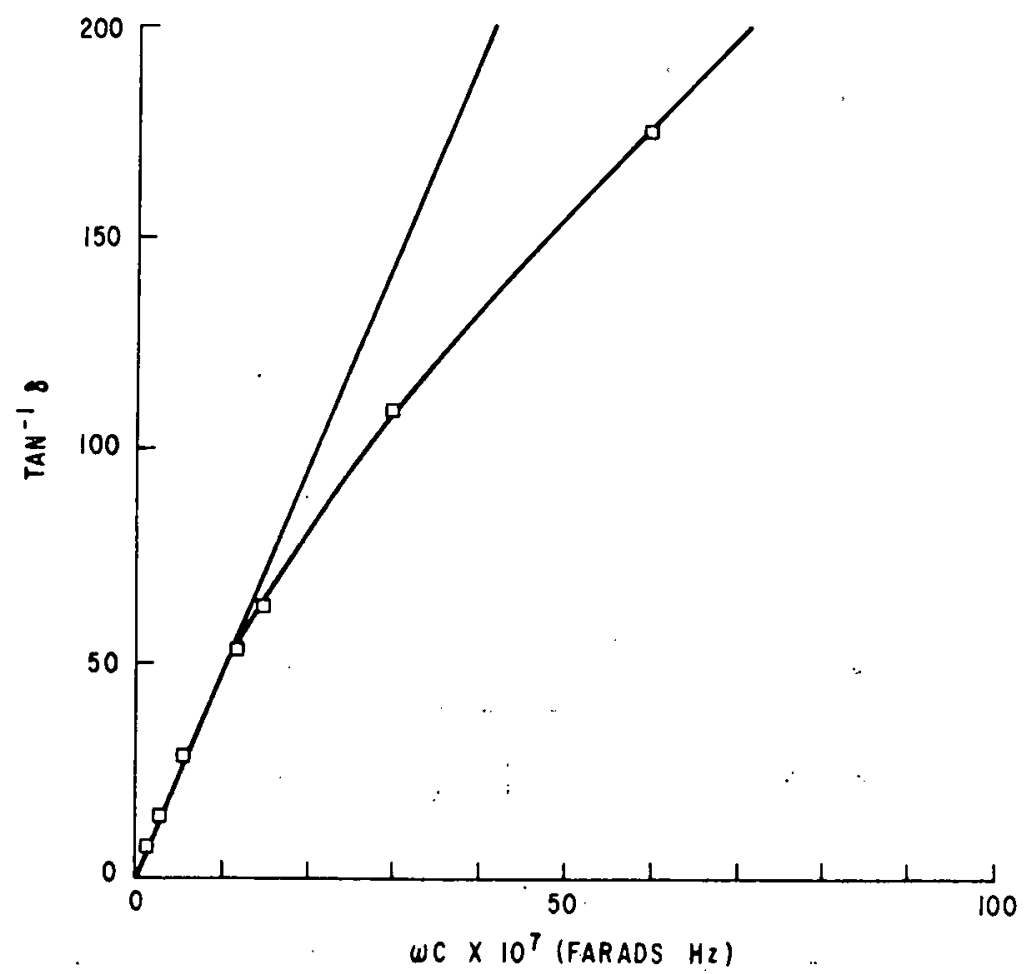

Fig. 7-15 Frequency dependence of reciprocal loss tangent. PVAP +4 응 AD48+

58\% Pyranol 1499. Thickness $3 \mathrm{mil}$. 


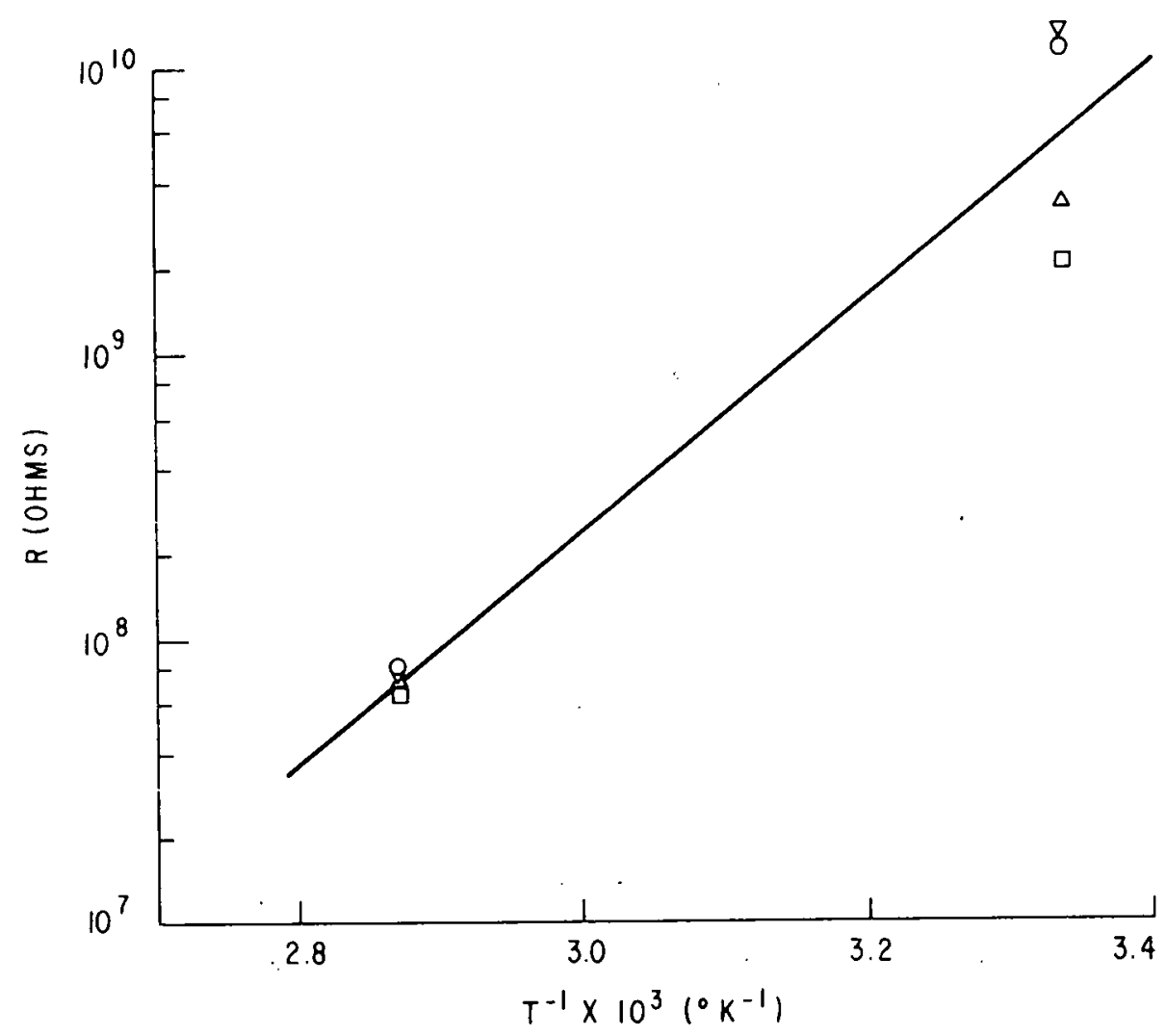

Fig. 7-16 Arrhenius plot. PVAP + 4\% AD48+58\% Pyranol 1499. Thickness $3 \mathrm{mil}$.

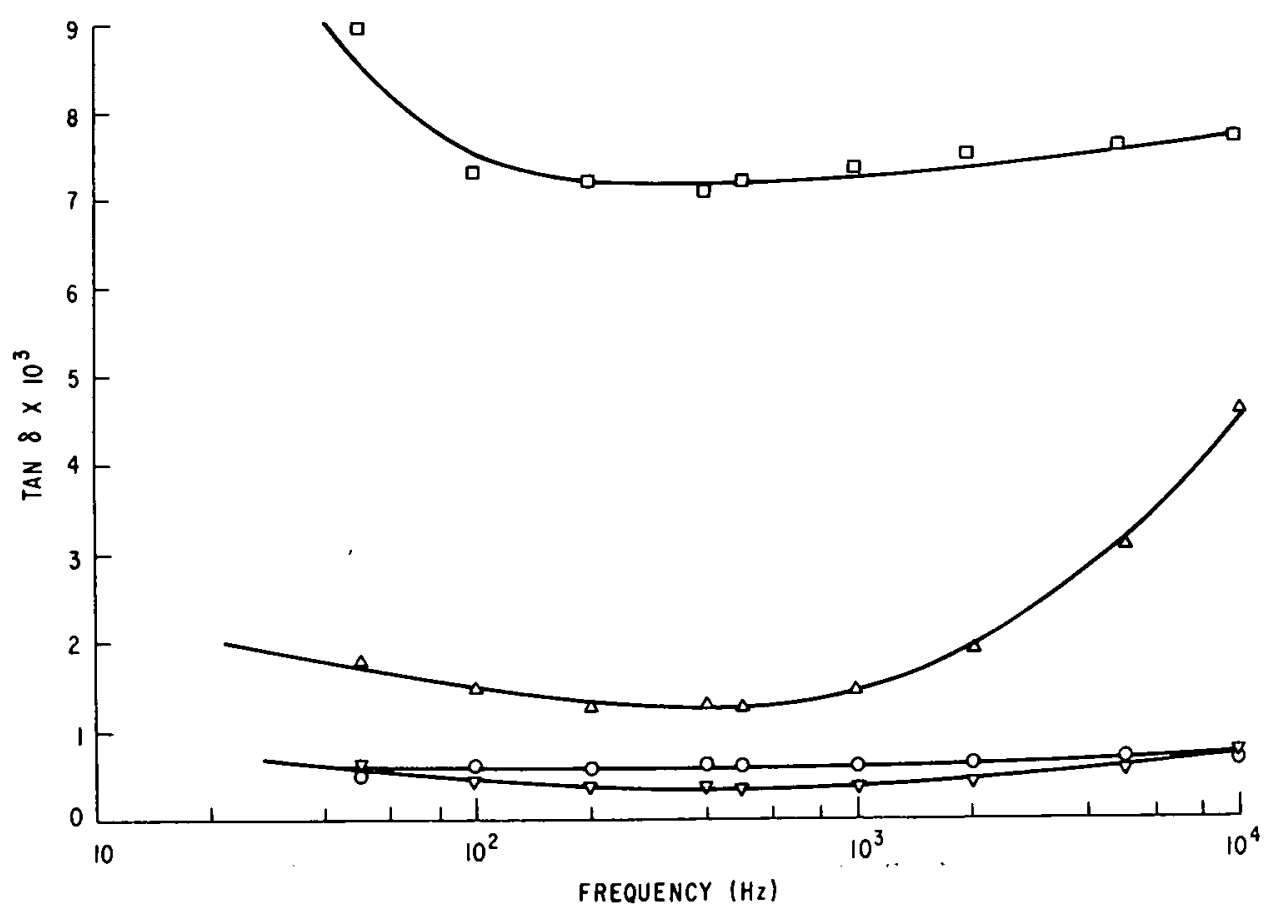

Fig. 7-17 Frequency dependence of loss tangent. $\mathrm{PE}+5 \%$ Lubrizol 796 . 


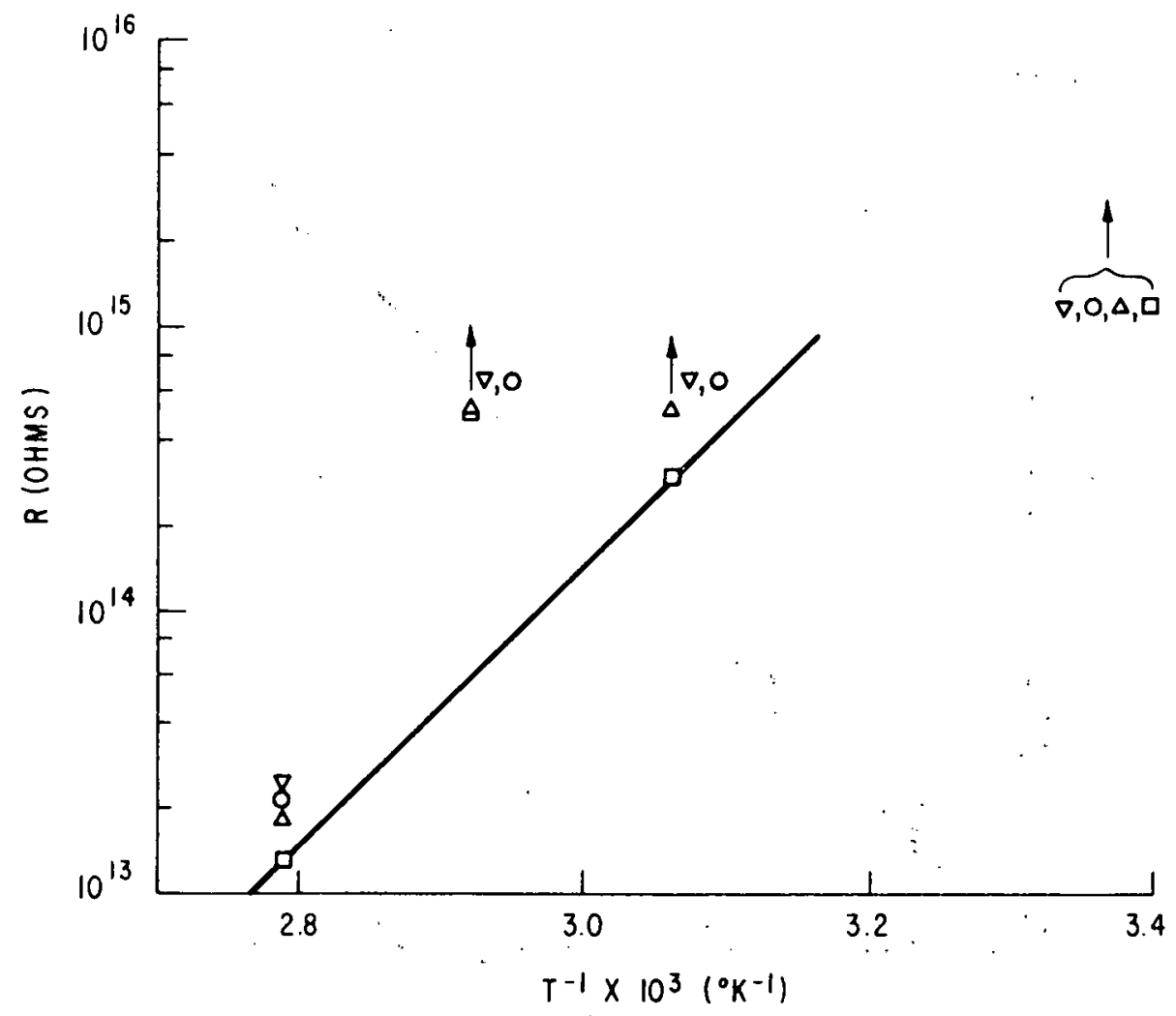

Fig. 7.-18 Arrhenius plot. PE + 5\% Lubrizol 796.

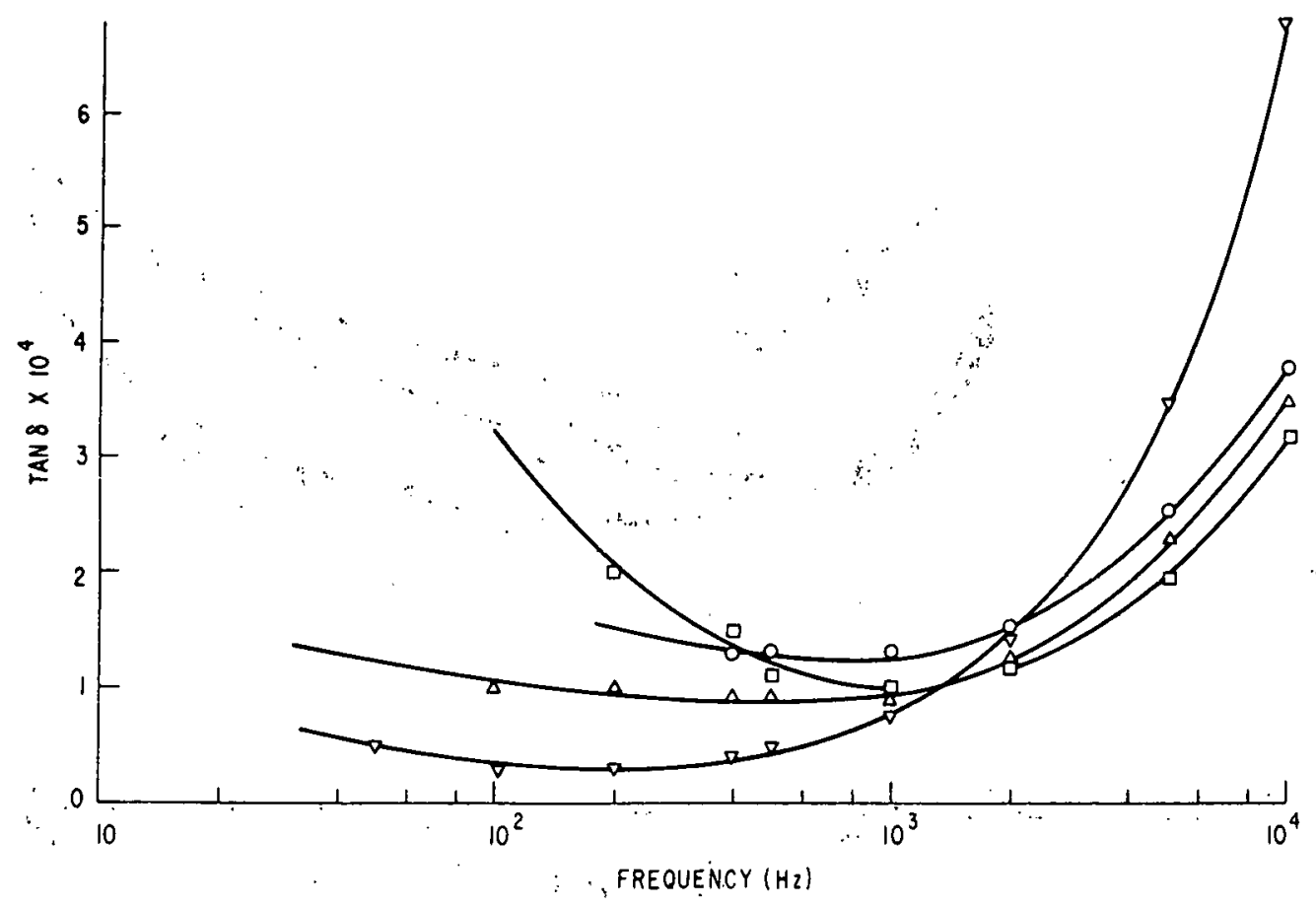

Fig. 7-19 Frequency dependence of loss tangent. $\mathrm{PE}+5 \%$ Lubrizol 74 . 


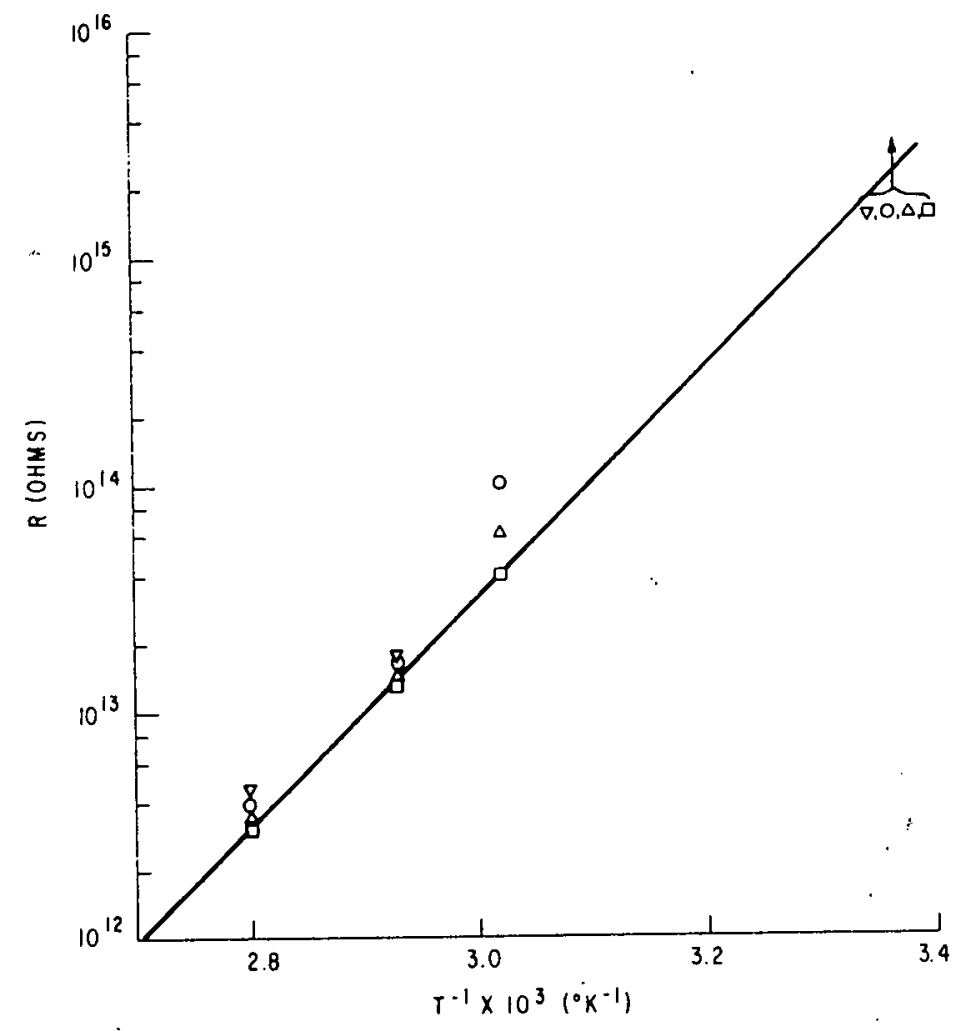

Fig. 7-20 Arrhenius plot. PE + 5\% Lubrizol 74.

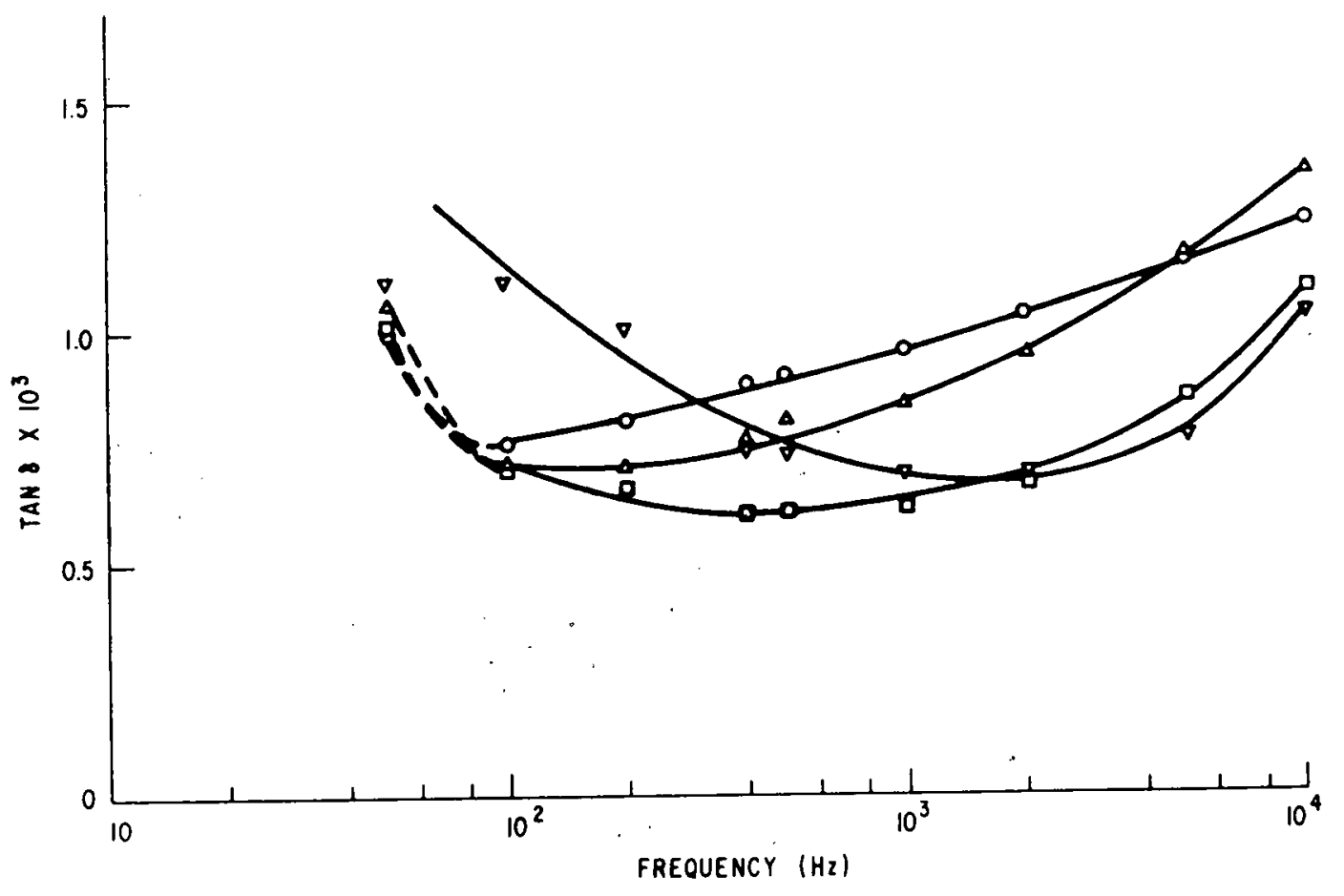

Fig. 7-21 Frequency dependence of loss tangent. $\mathrm{PE}+5 \%$ Lubrizol 52 . 


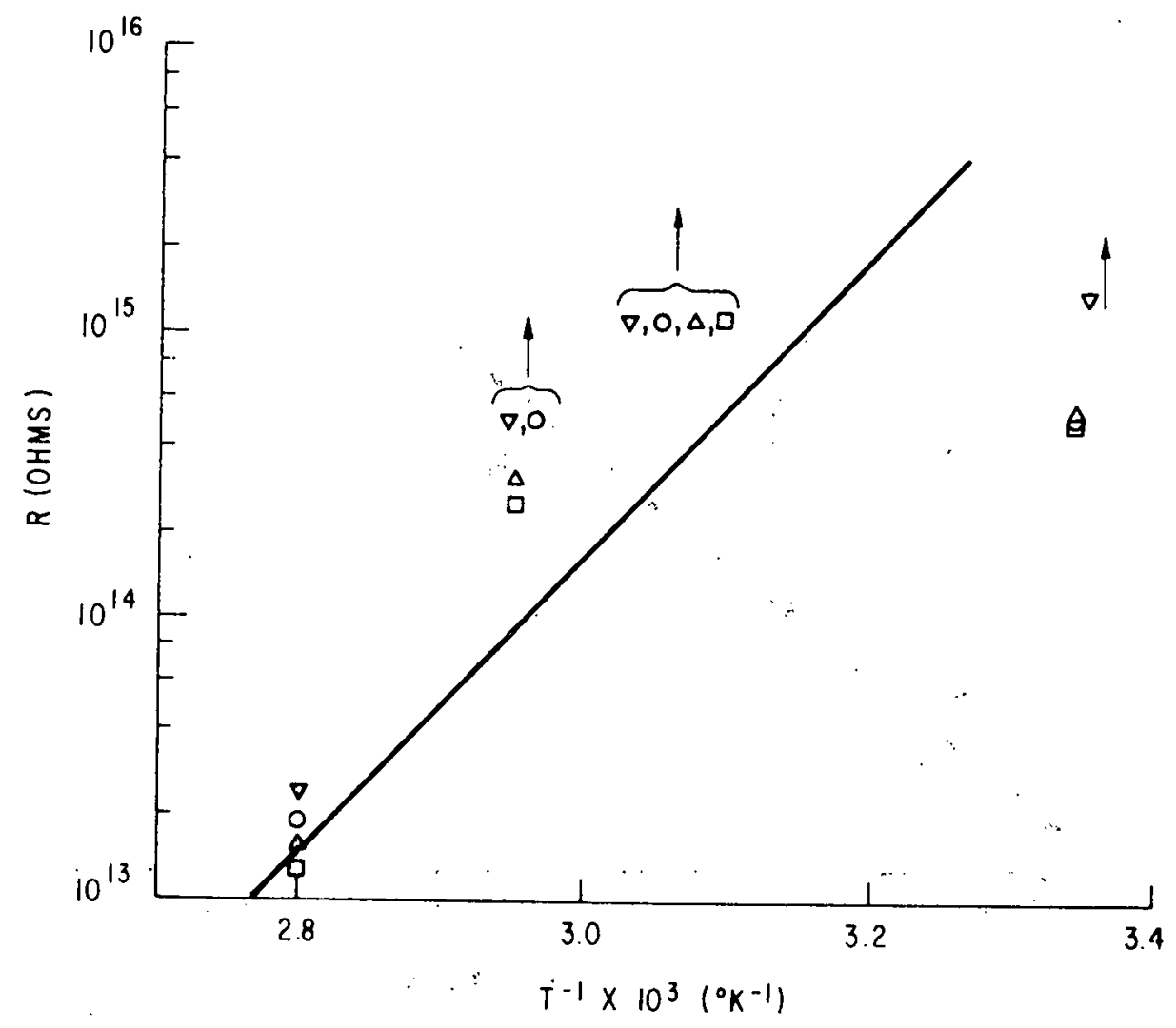

Fig. 7-22 Arrhenius plot. : PE + .5\% Lubrizol 52.

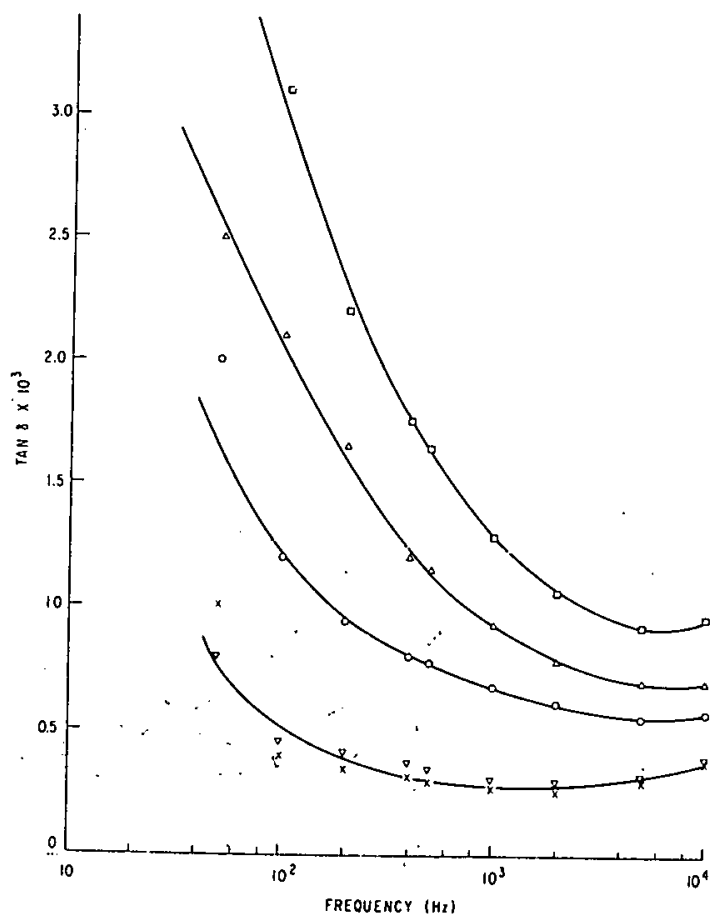

Fig. 7-23 Frequency dependence of loss tangent. $\mathrm{PE}+5 \%$ Lubrizol $25352 \mathrm{E}$. ( $\mathrm{x}$ ) $25^{\circ} \mathrm{C}$ after heat cycle to $85^{\circ} \mathrm{C}$. 


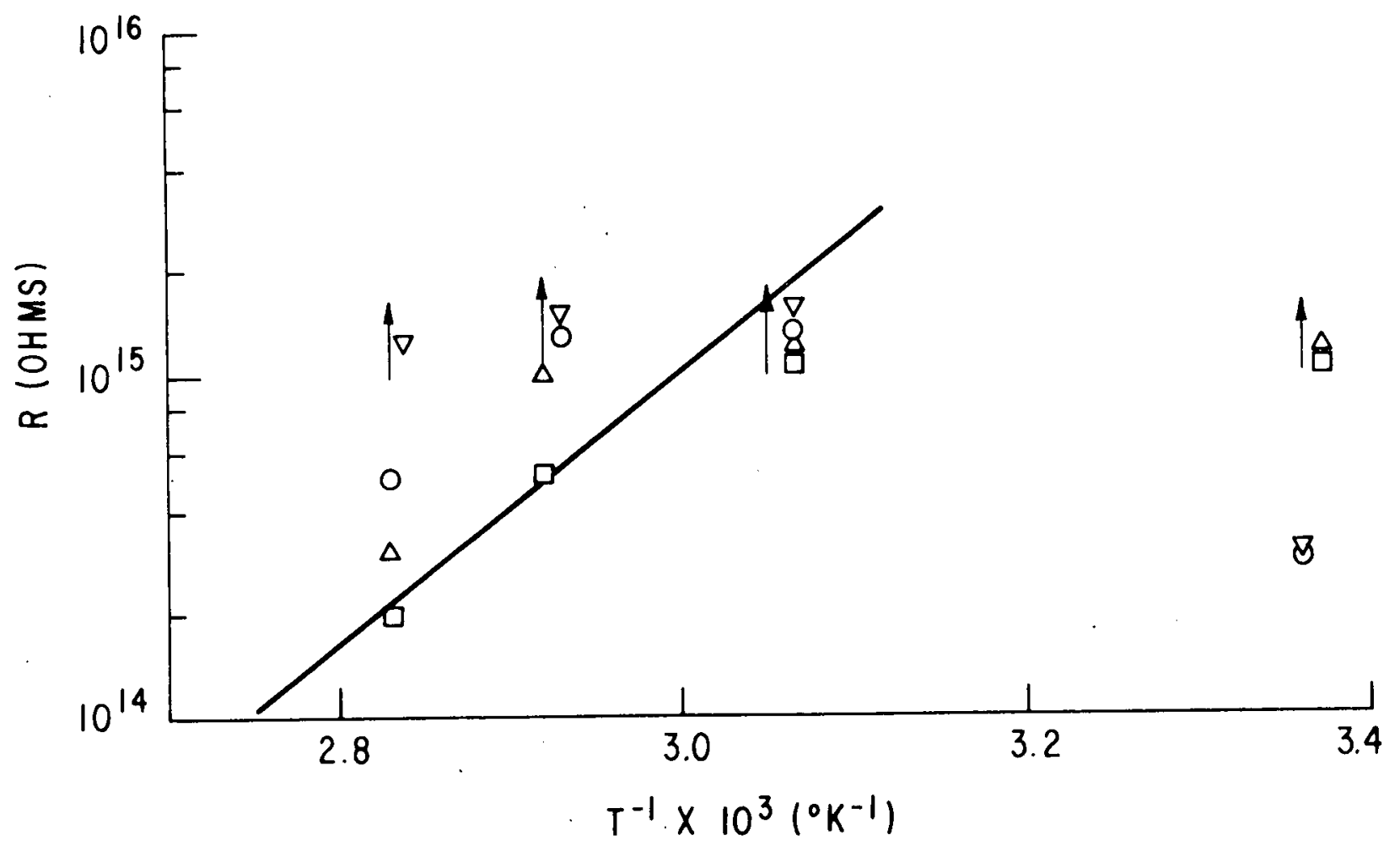

Fig. 7-24 Arrhenius plot: PE + 5\% Lubrizol 25352E.

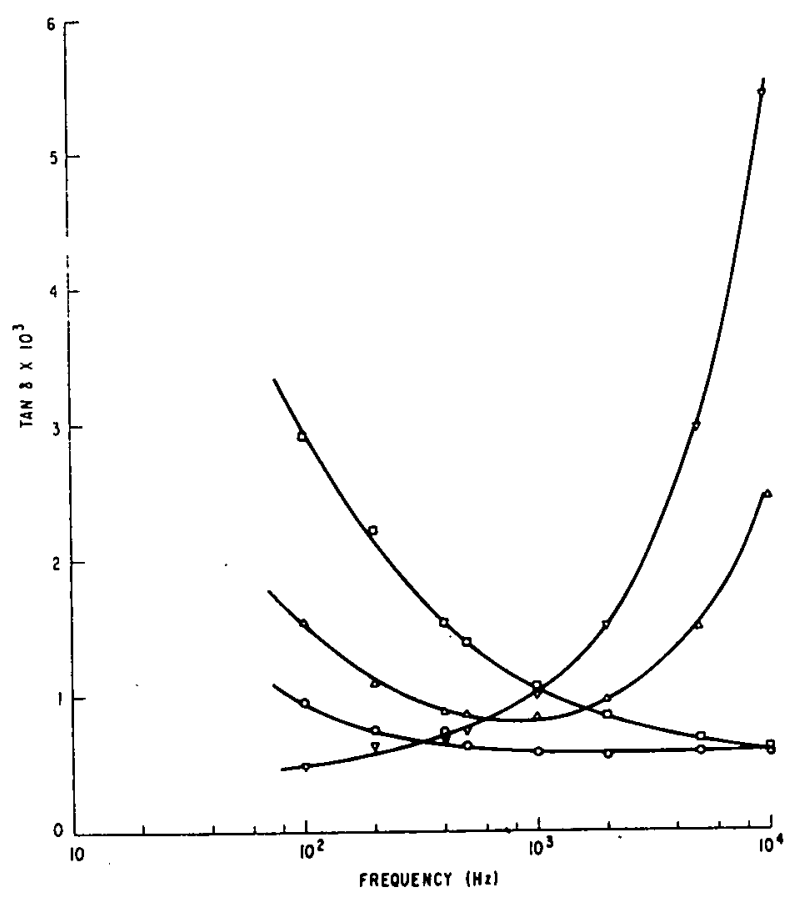

Fig. 7-25, Frequency dependence of loss tangent. $\mathrm{PE}+5 \%$ Lubrizol 25352E + 10\% Pyranol 1499. 


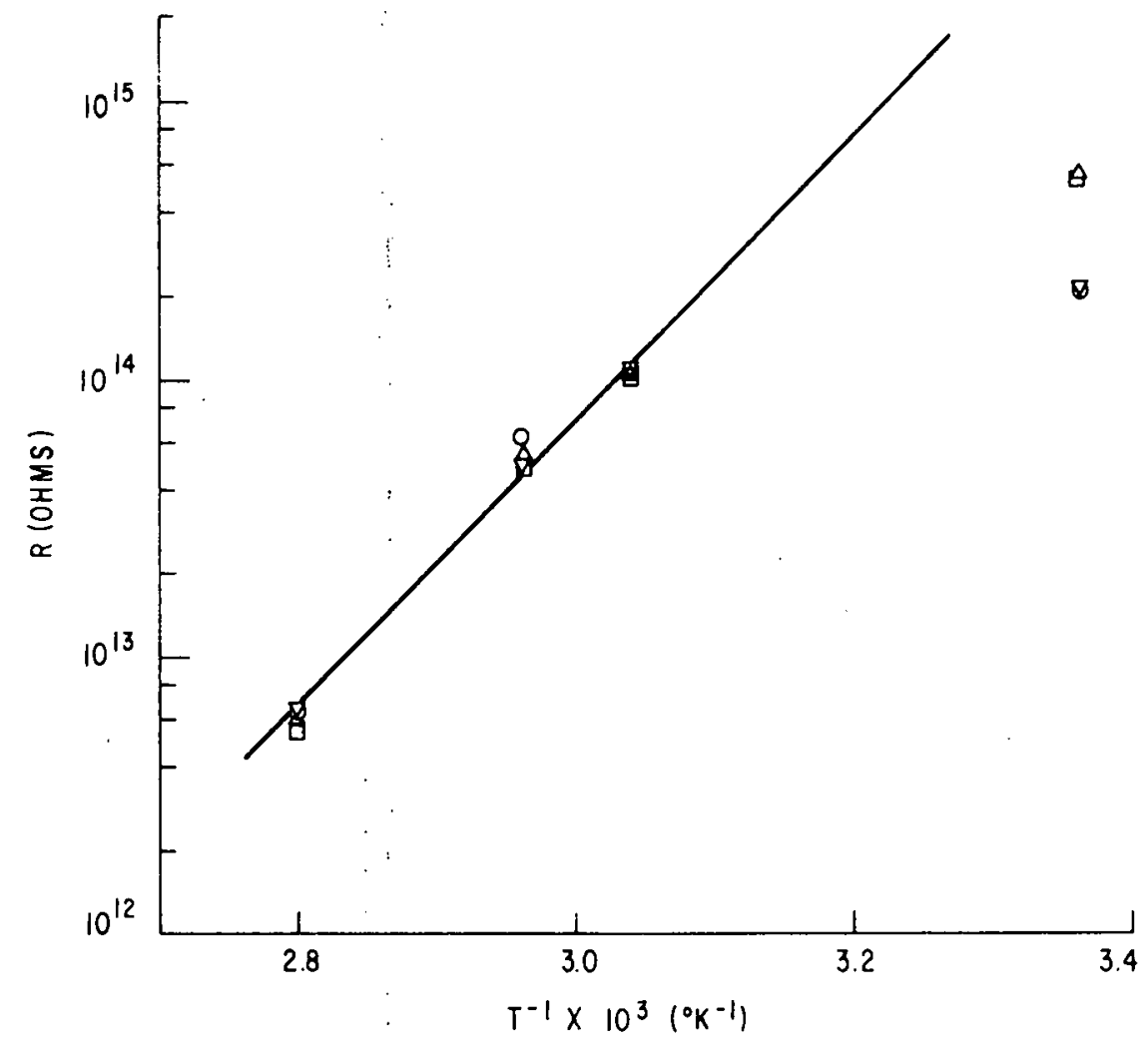

Fig. 7-26 Arrhenius plot. $P E+5 \%$ Lubrizol 25352E + 10\% Pyranol 1499.

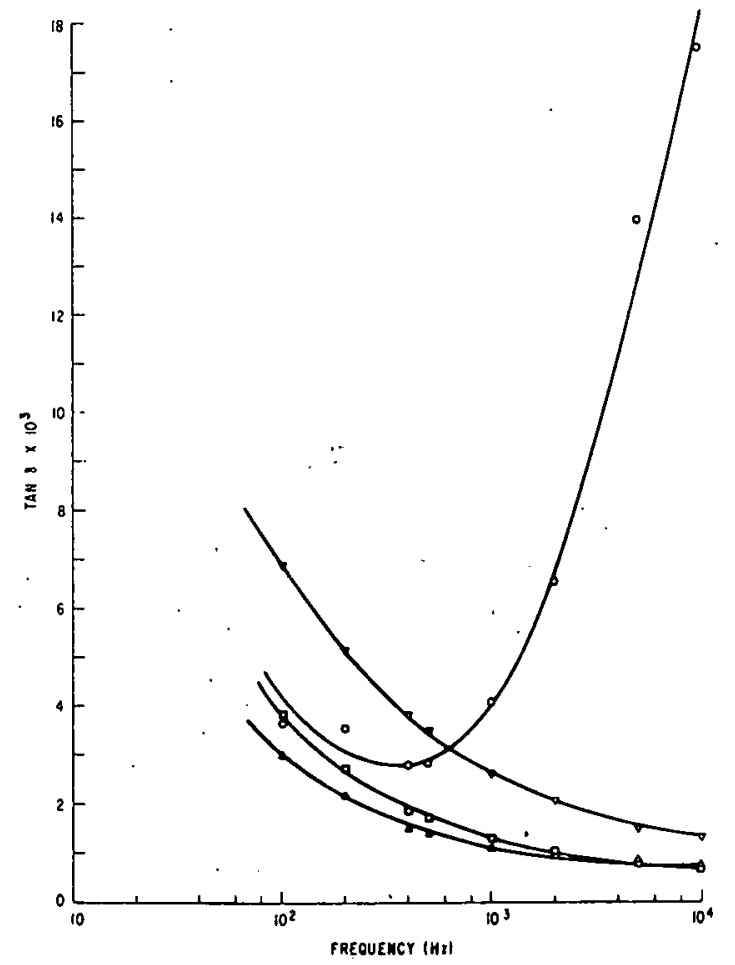

Fig. 7-27 Frequency dependence of loss tangent. $\mathrm{PE}+5 \%$ Lubrizol + 3\% Pyranol 1499. 


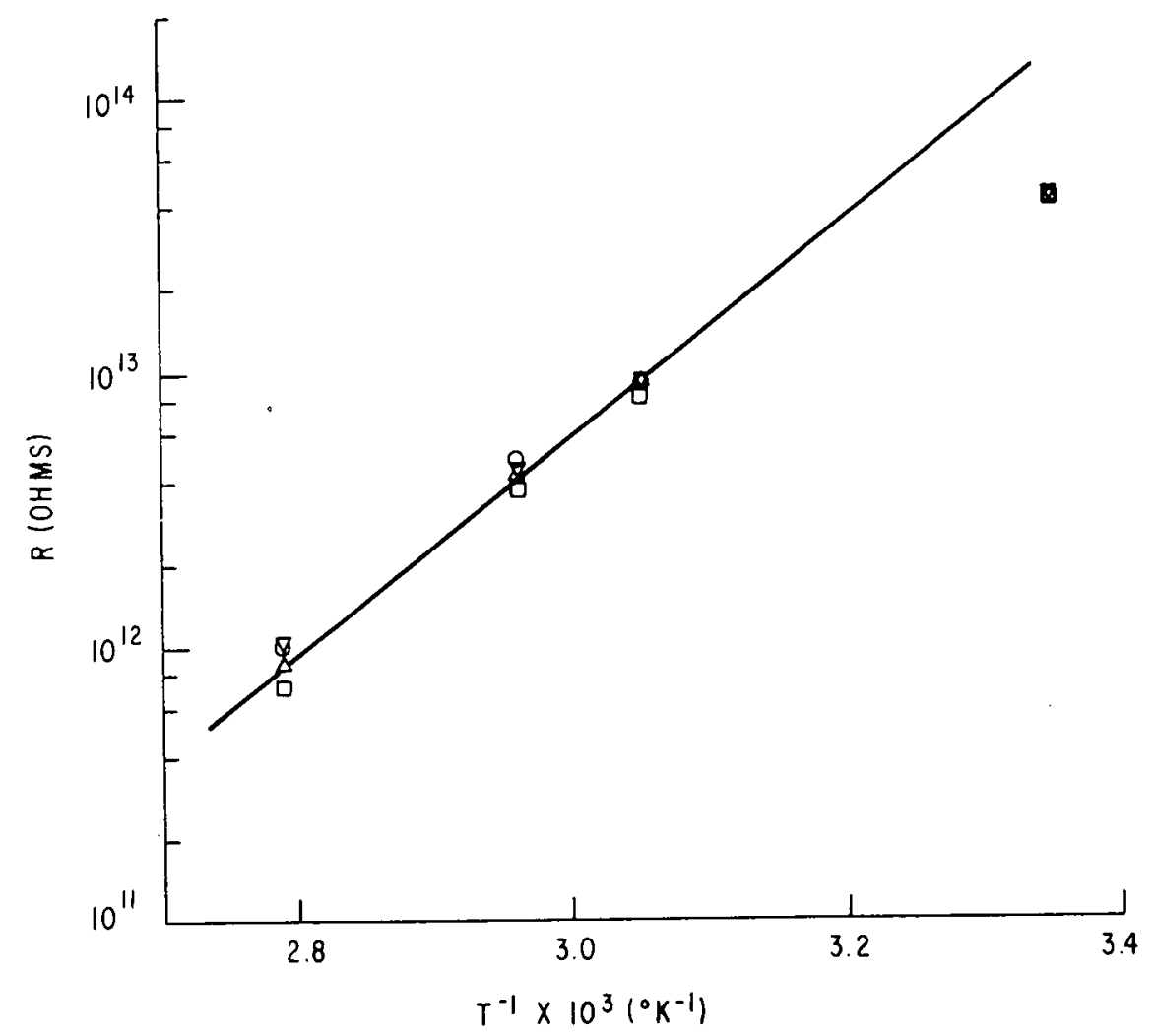

Fig. 7-28 Arrhenius plot. PE + 5\% Lubrizol + 30\% Pyranol 1499.

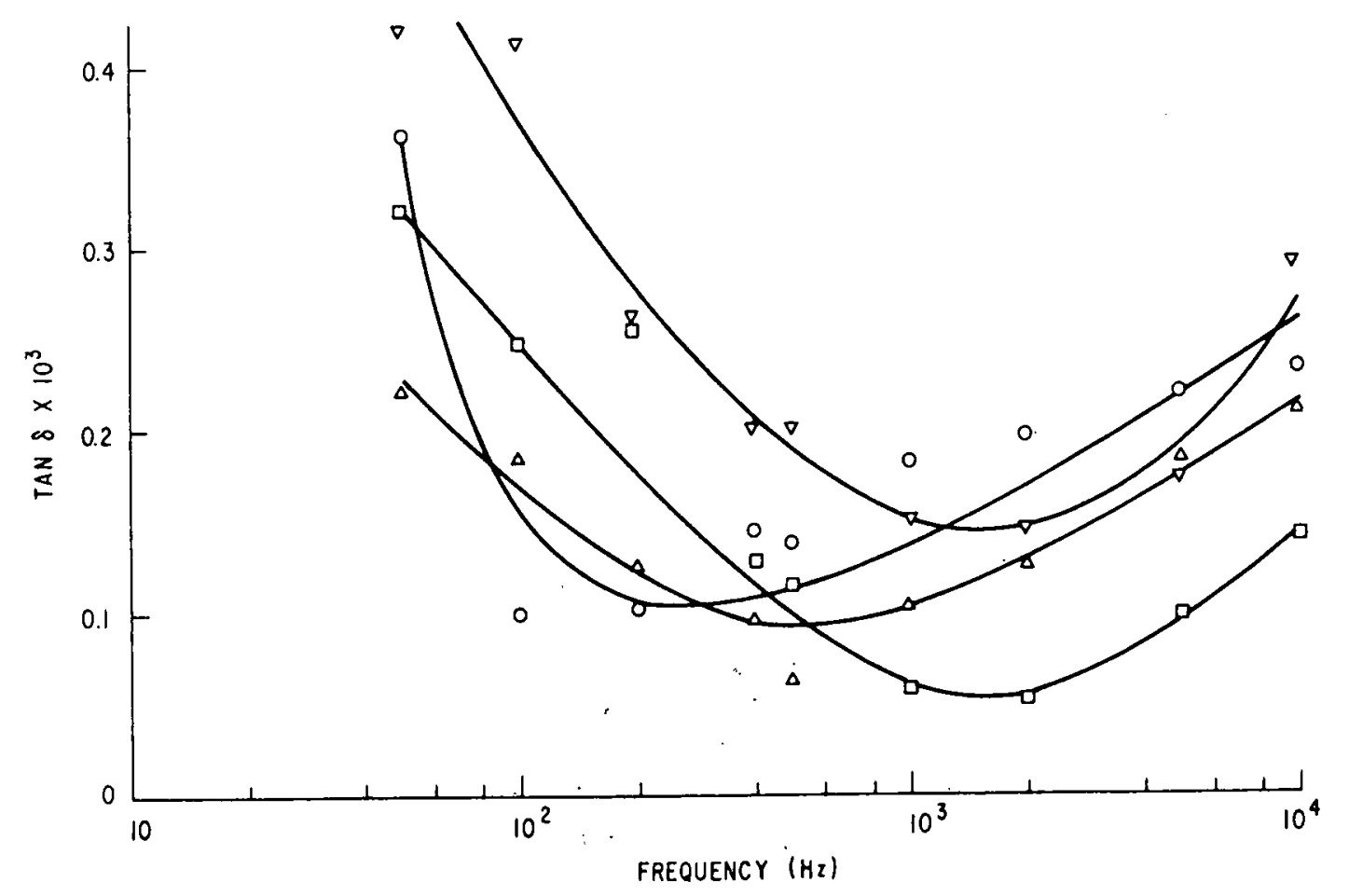

Fig. 7-29 Frequency dependence of loss tangent. $\mathrm{PE}+5 \dot{\circ}$ lithium stearate. 


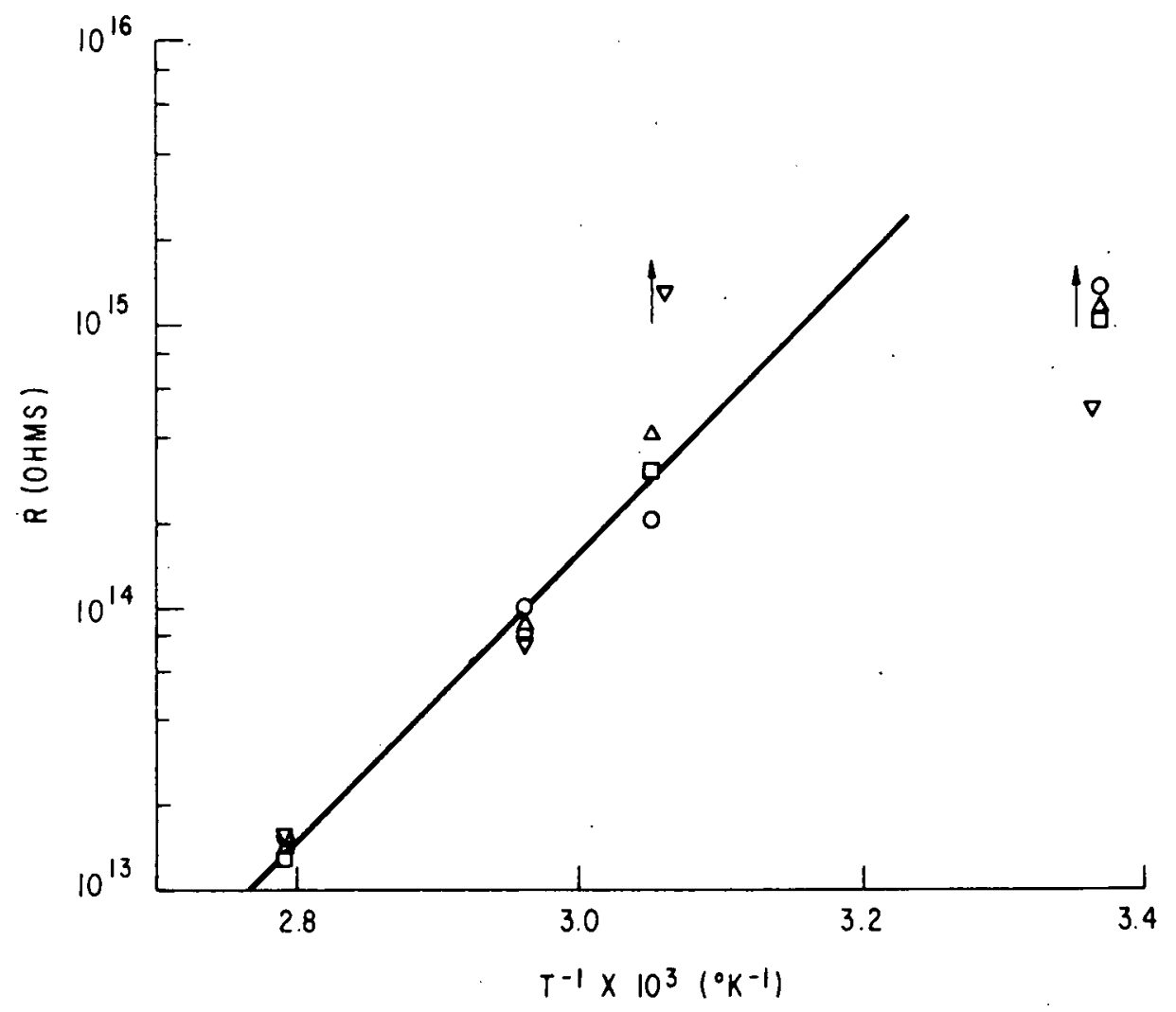

Fig. 7-30 Arrhenius plot. $P E+5 \%$ lithium stearate.

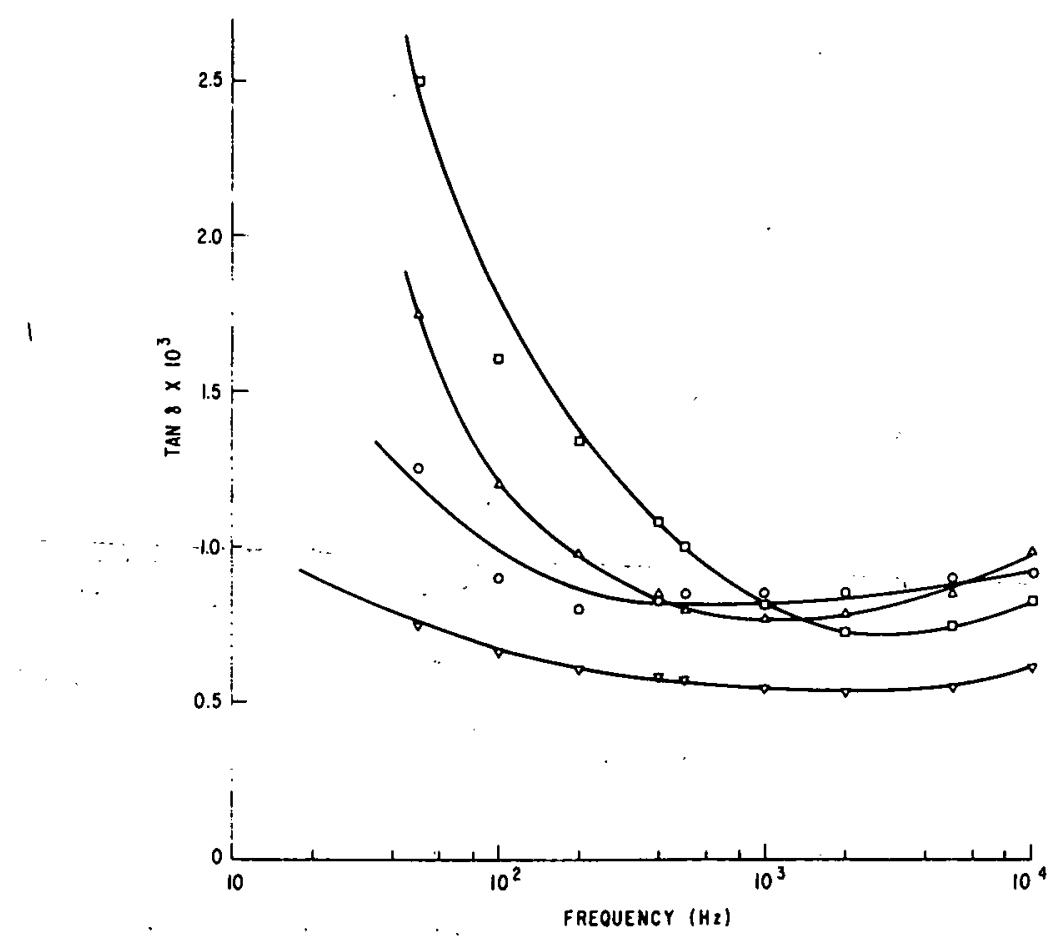

Fig. 7-31 Frequency dependence of loss tangent. $\mathrm{PE}+5 \%$ sodium stearate. 


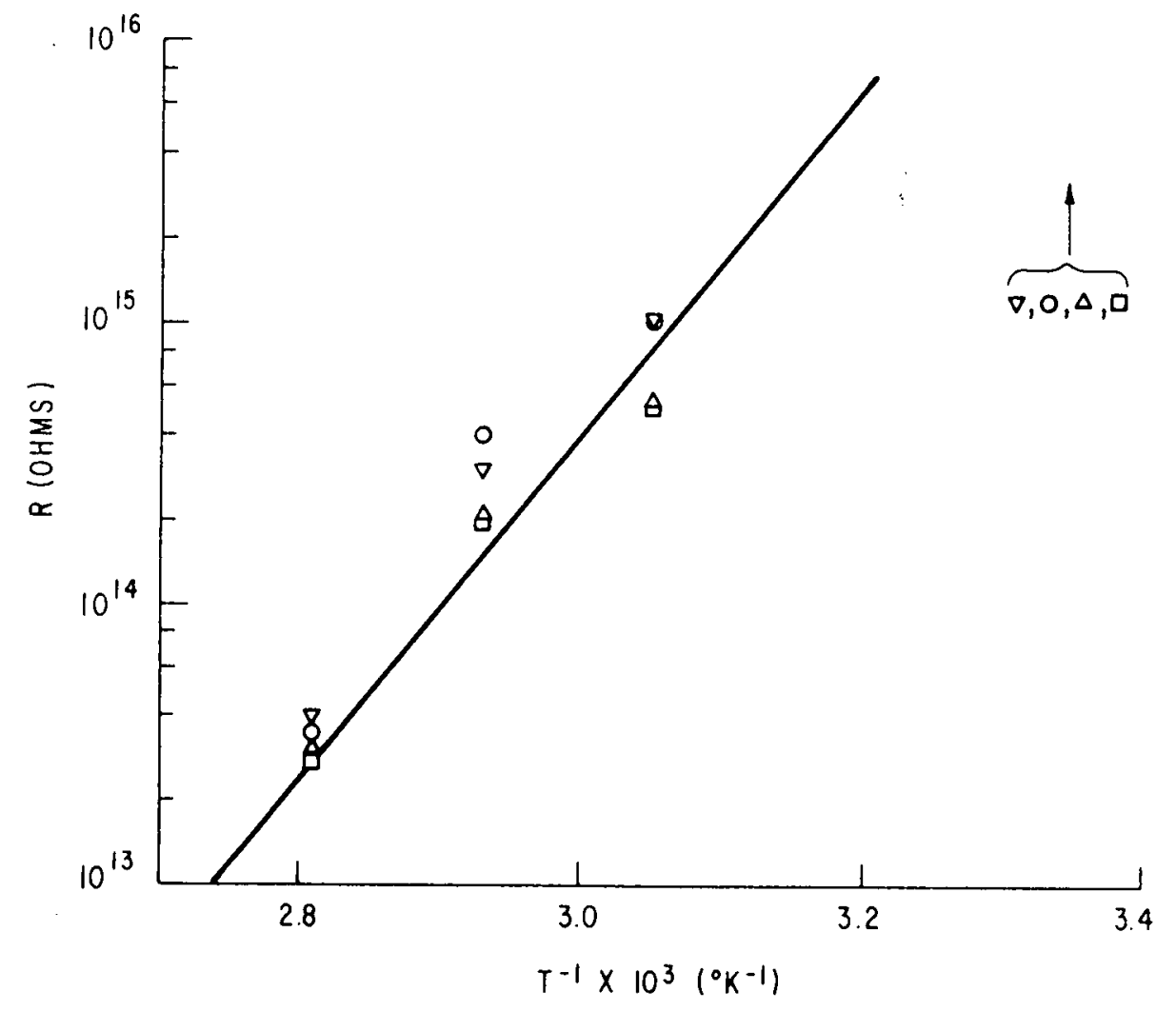

Fig. 7-32 Arrhenius plot. $\mathrm{PE}+5 \%$ sodium stearate.

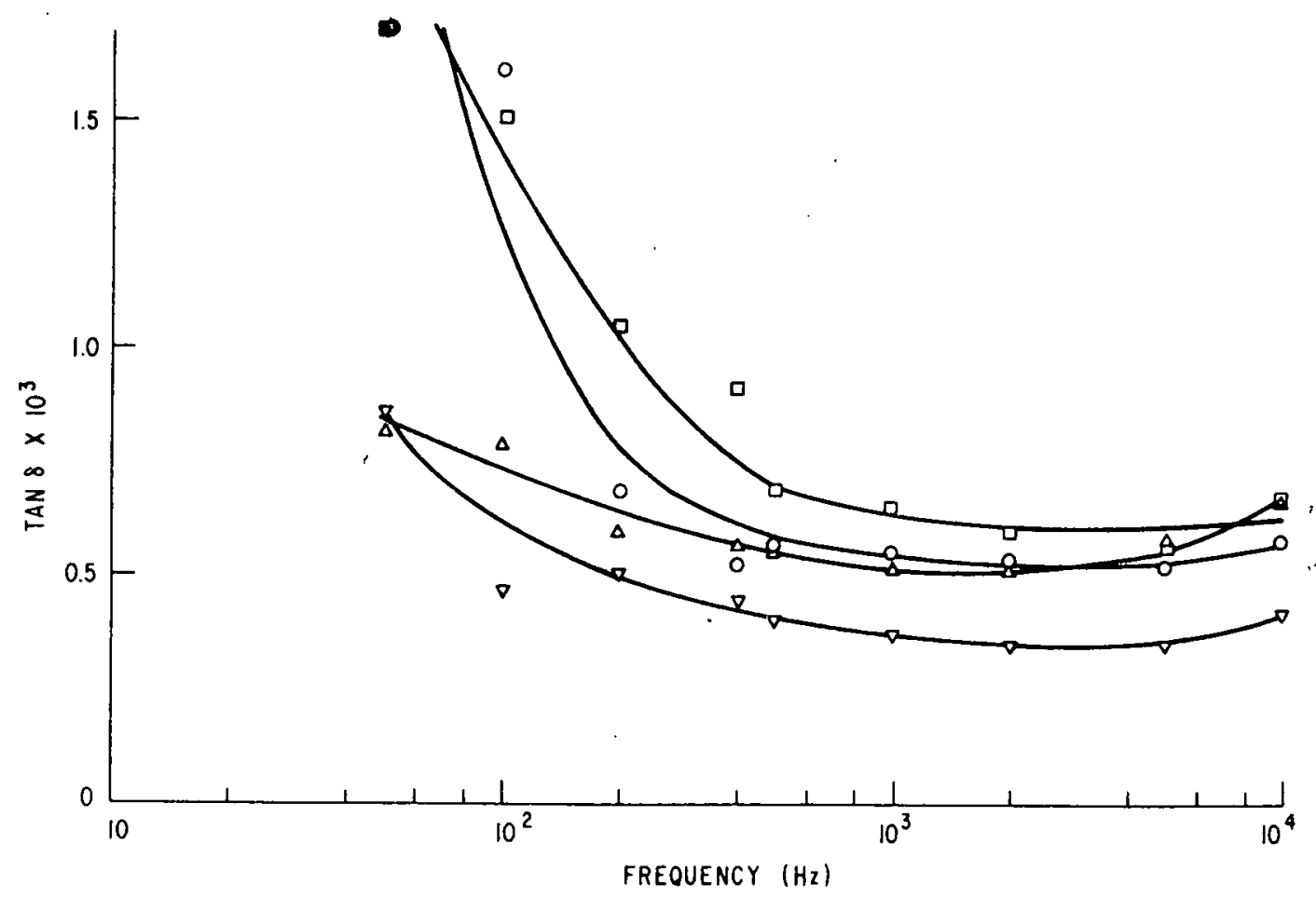

Fig. 7-33 Frequency dependence of loss tangent. $\mathrm{PE}+5$ o potassium stearate. 


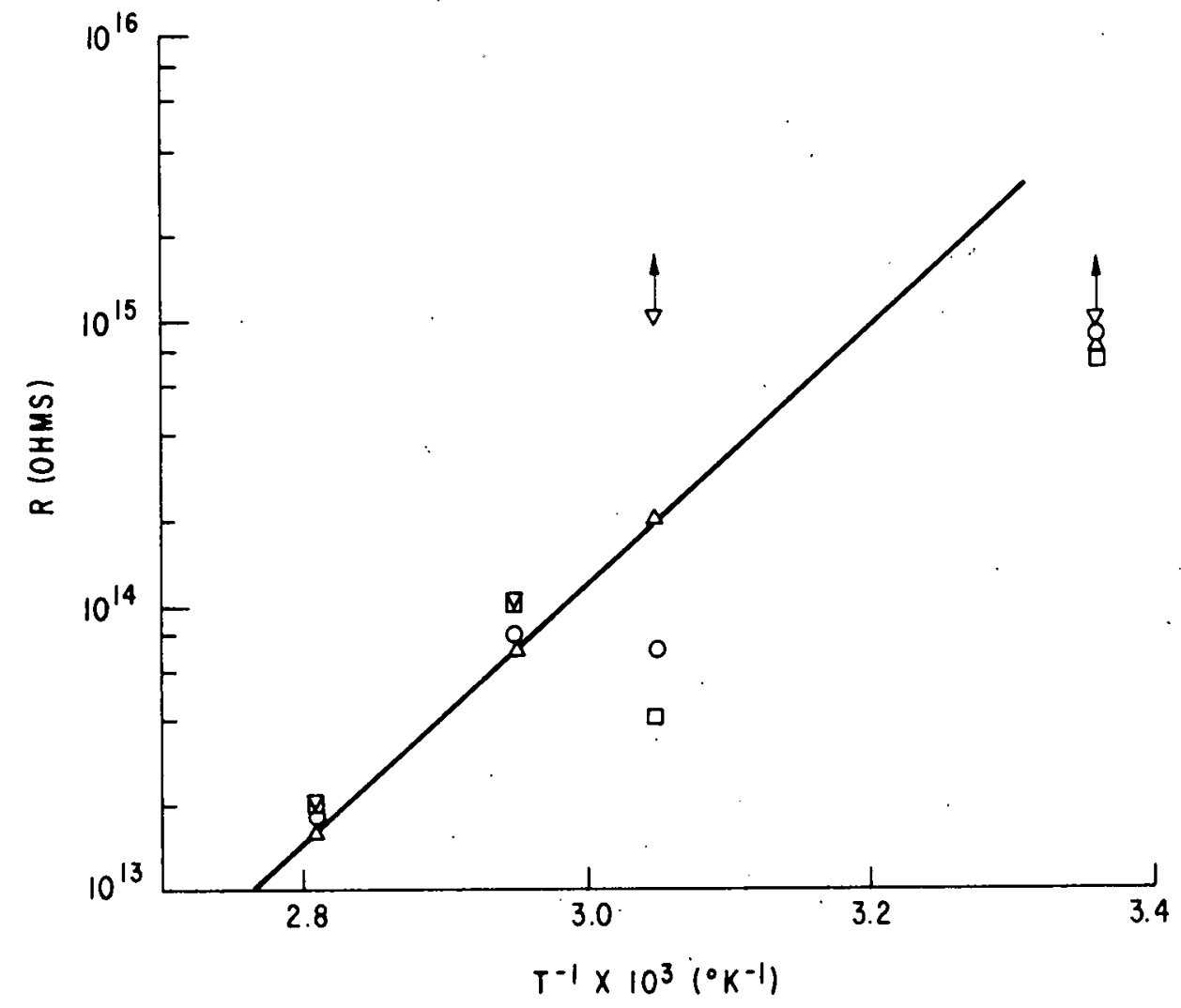

Fig. 7-34 Arrhenius plot. PE + 5\% potassium stearate.

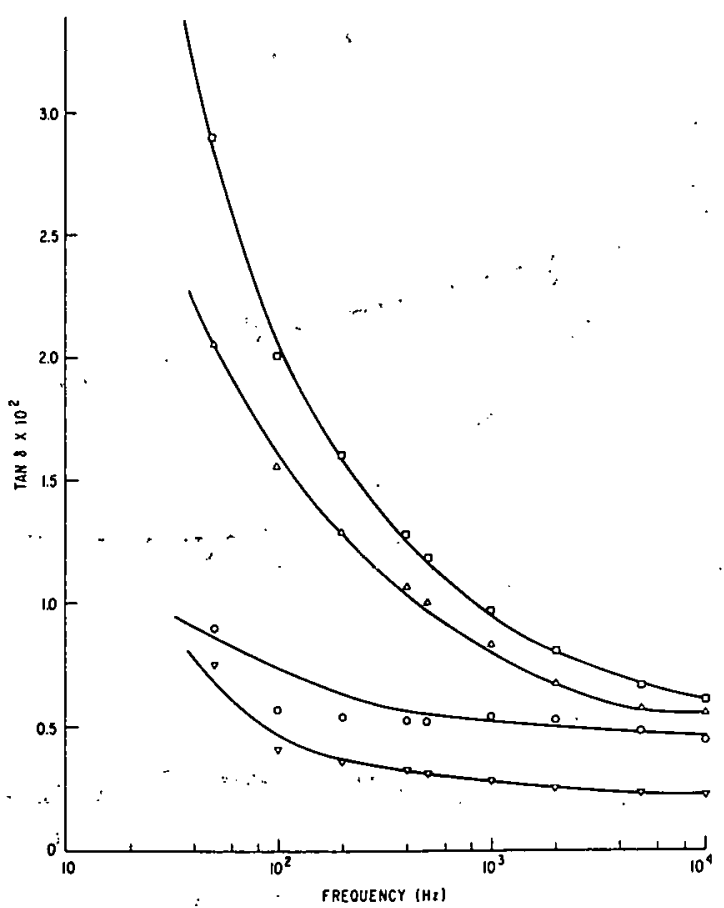

Fig. 7-35 Frequency dependence of loss tangent. $\mathrm{PE}+5 \%$ tetramethyl ammonium stearate. 


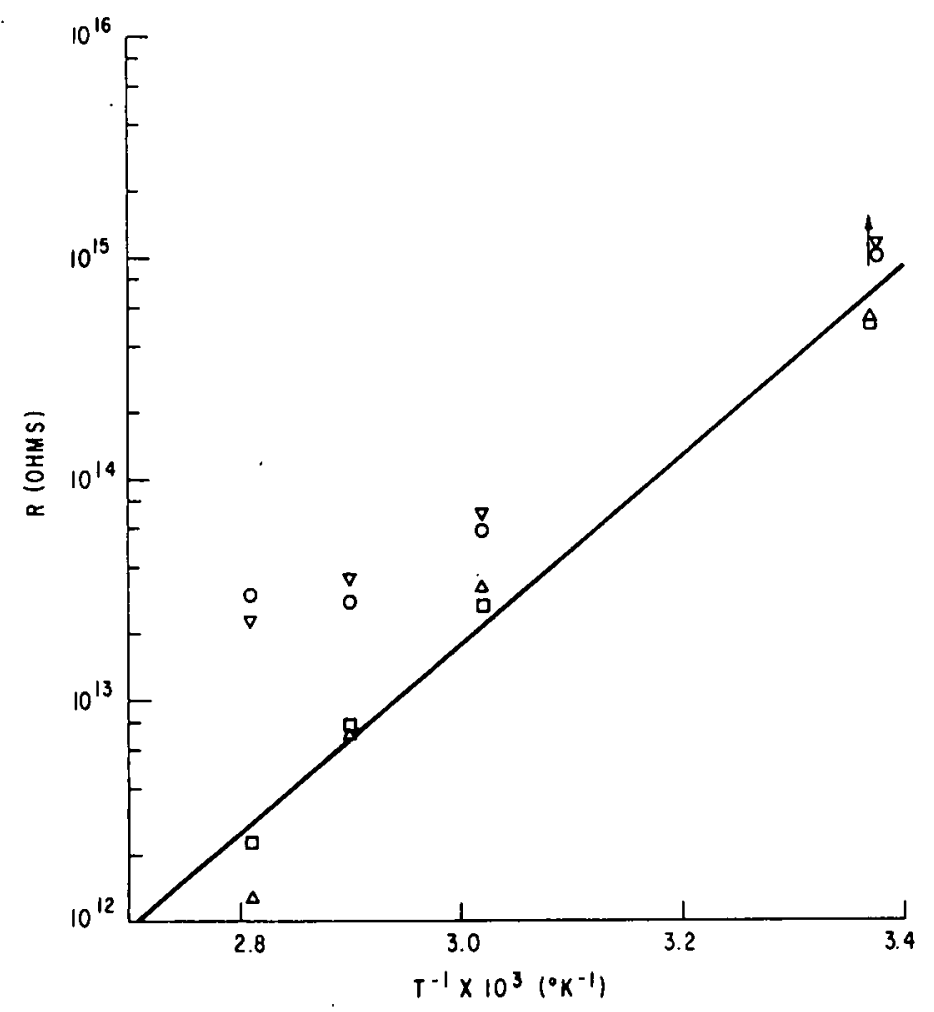

Fig. 7-36 Arrhenius plot. $P E+5 \%$ tetramethyl ammonium stearate.

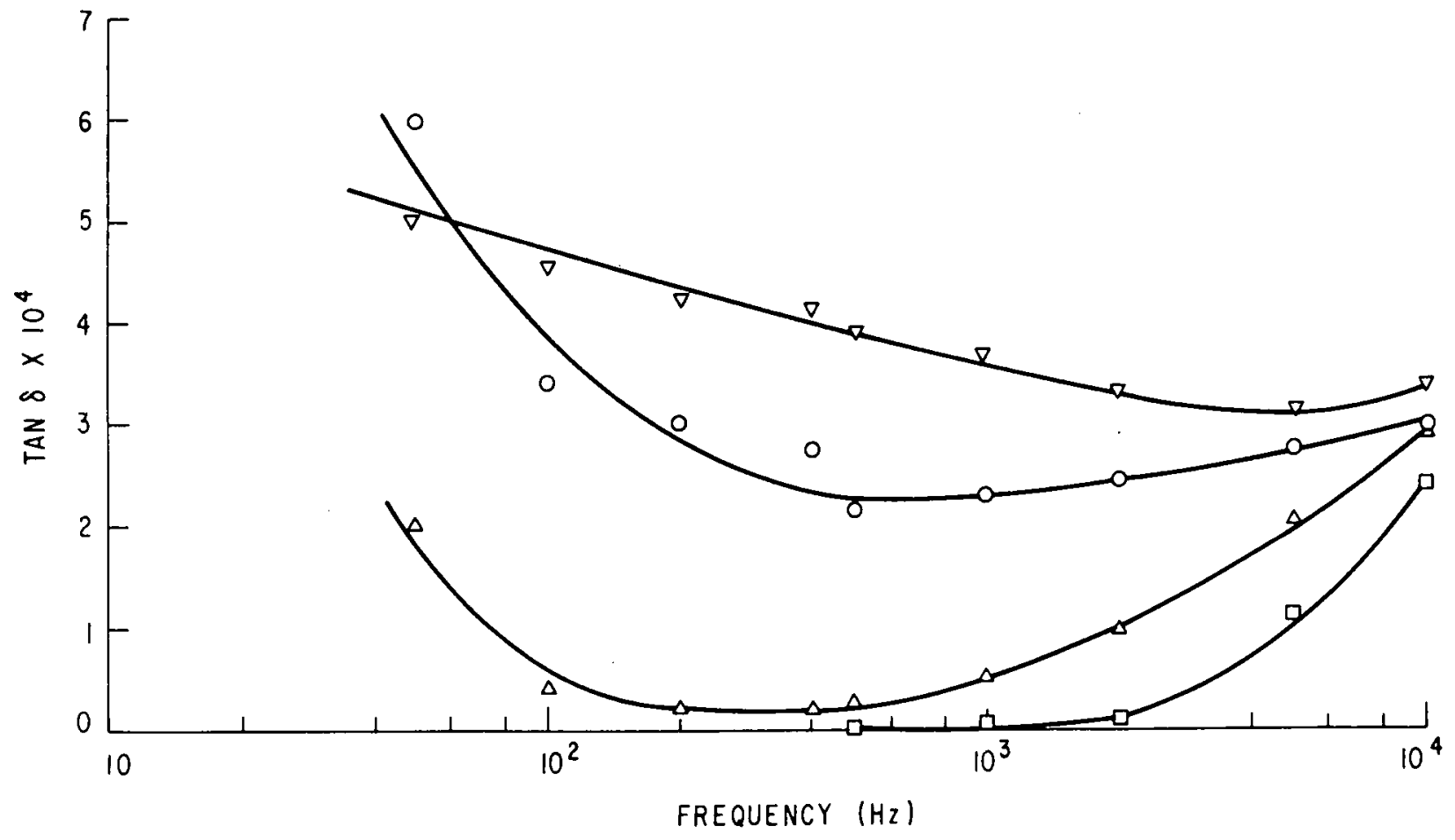

Fig. 7-37 Frequency dependence of loss tangent. $\mathrm{PE}+5 \%$ myristic acid. 


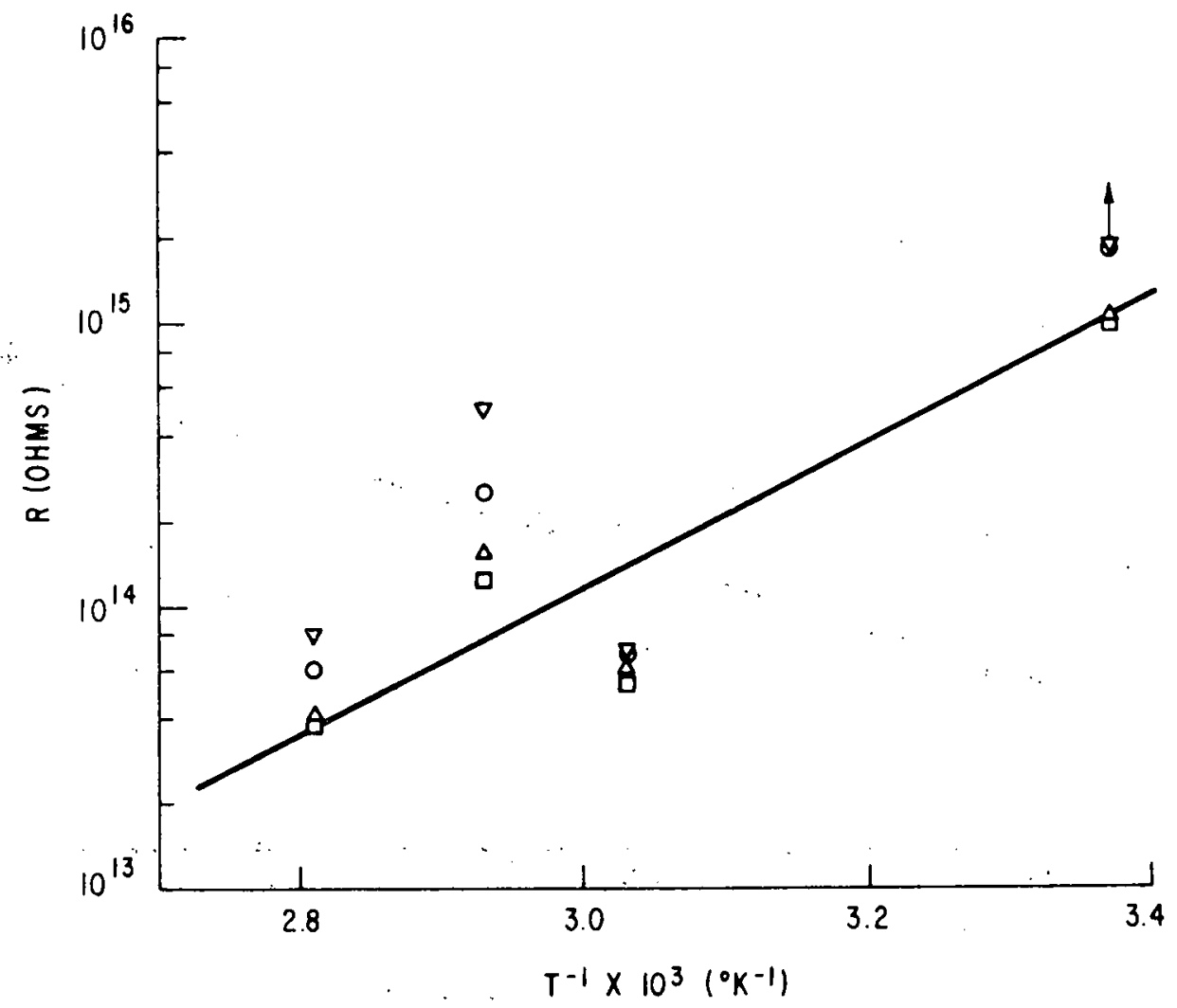

Fig. 7-38 Arrhenius plot. $P E+5 \%$ myristic acid.

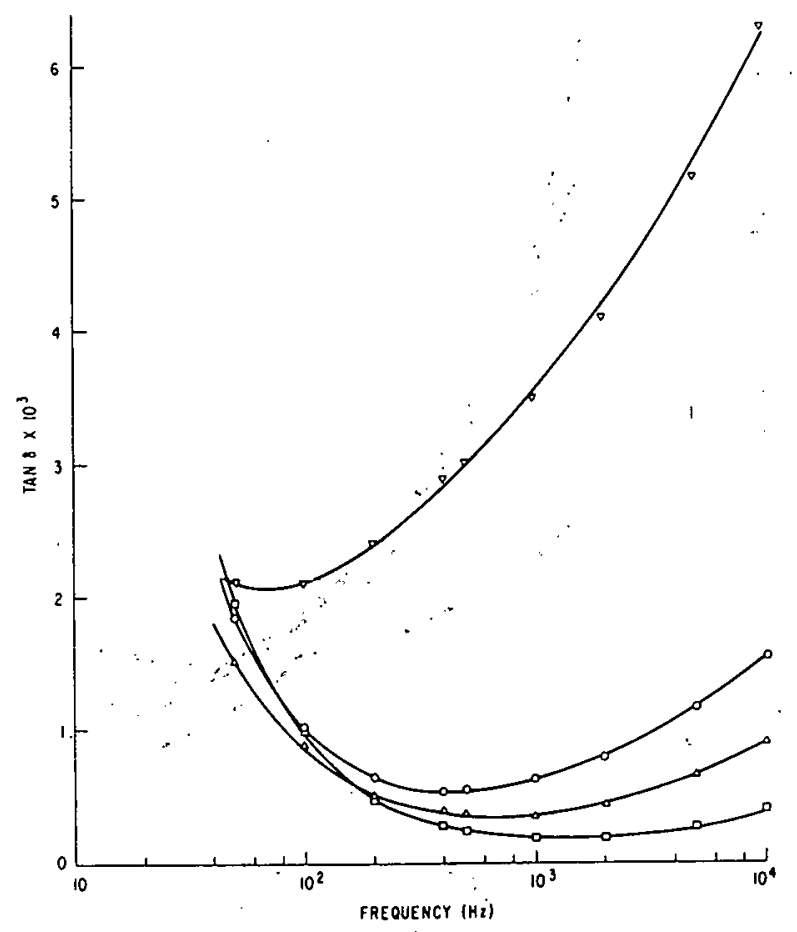

Fig. 7-39 Frequency dependence of loss tangent. PVAP + 5\% myristic acid. 


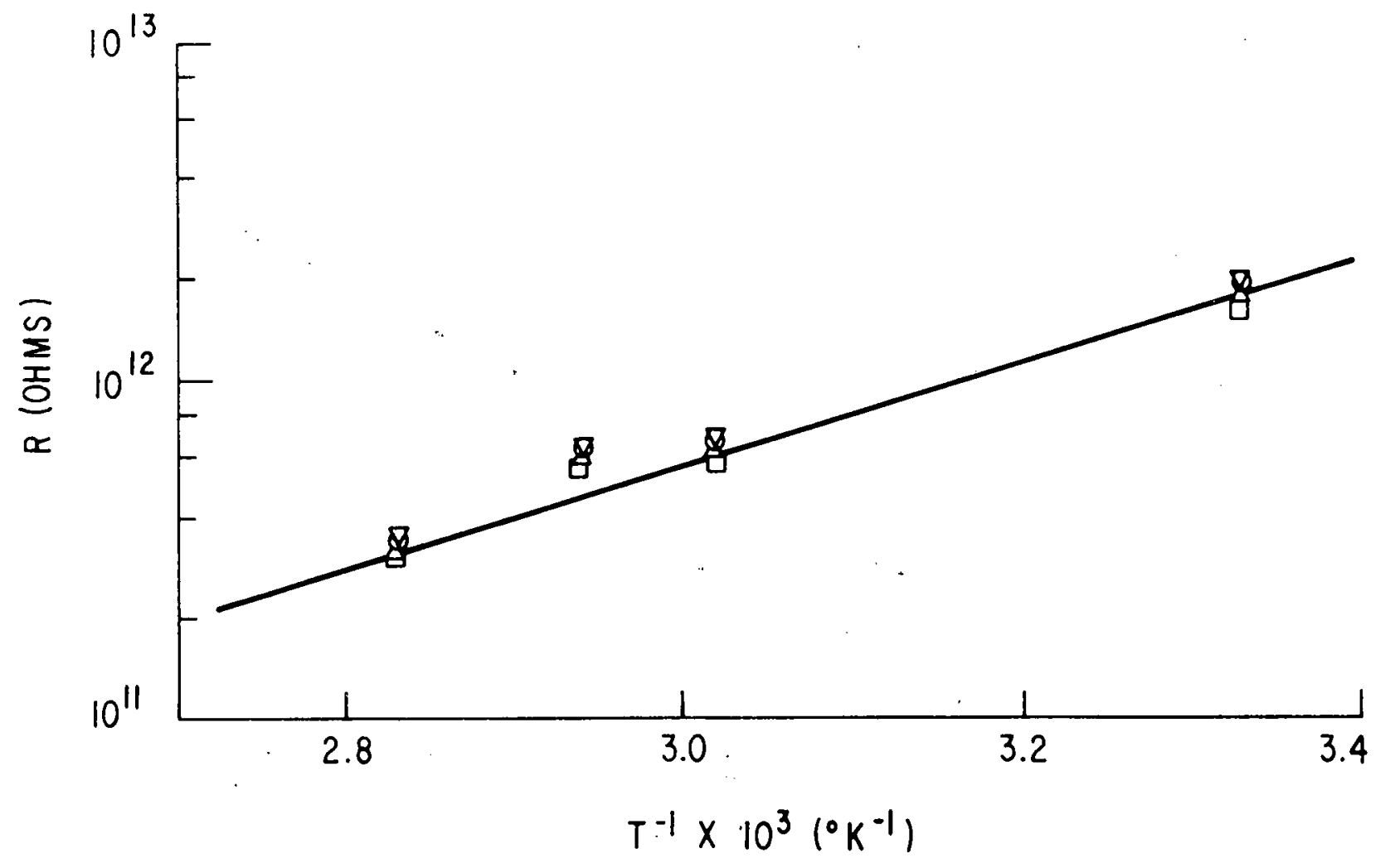

Fig. 7-40 Arrhenius plot. PVAP + 5\% myristic acid.

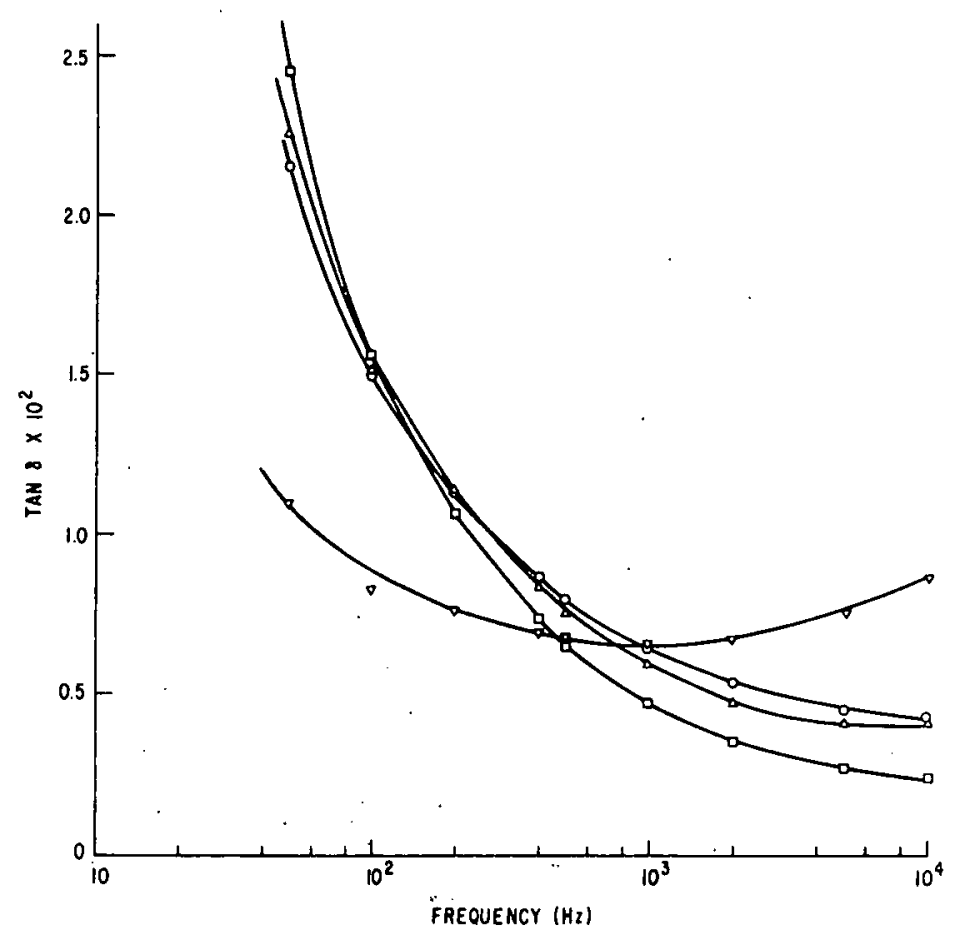

Fig: 7-4I Frequency dependence of loss tangent. PVAP + 5\% sodium oleate. 


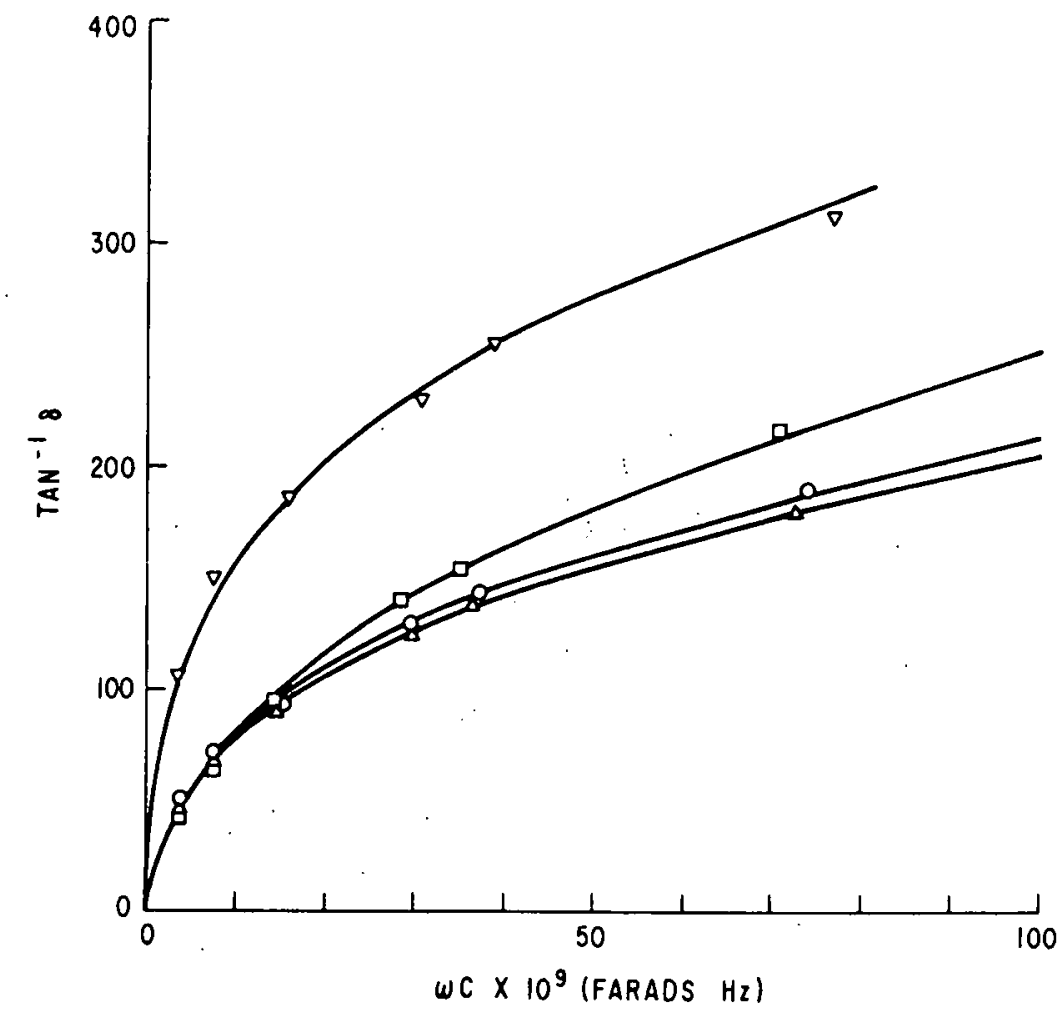

Fig. 7-42 Frequency dependence of the reciprocal loss tangent. PVAP $+5 \%$ sodium oleate.

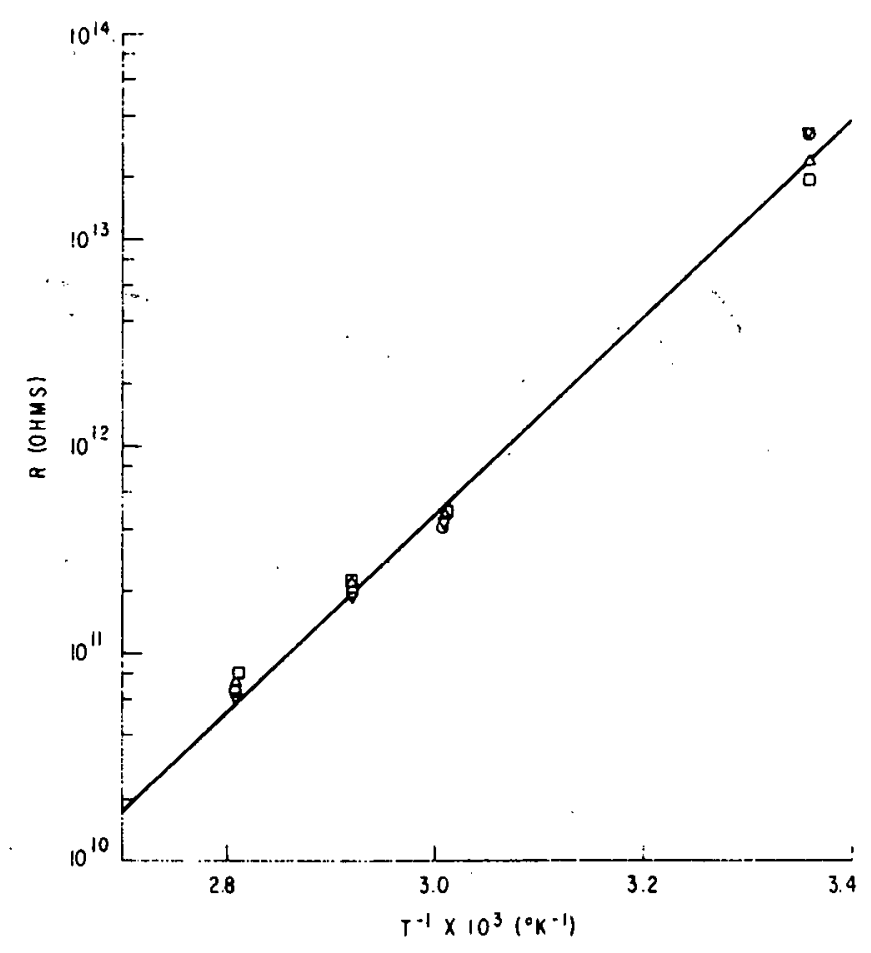

Fig. 7-43 Arrhenius plot. PVAP + 58 sodium oleate. 


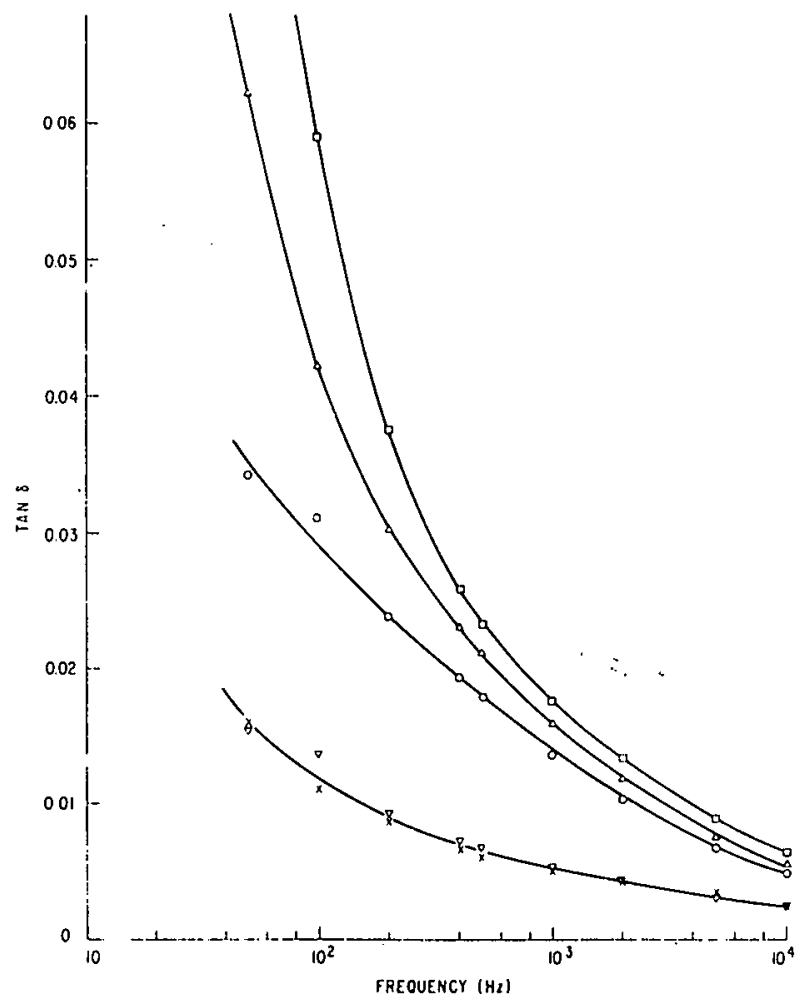

Fig. 7-44 Frequency dependence of the loss tangent. PE + 5q sodium lauryl sulfate. (x) $25^{\circ} \mathrm{C}$ after temperature cycling.

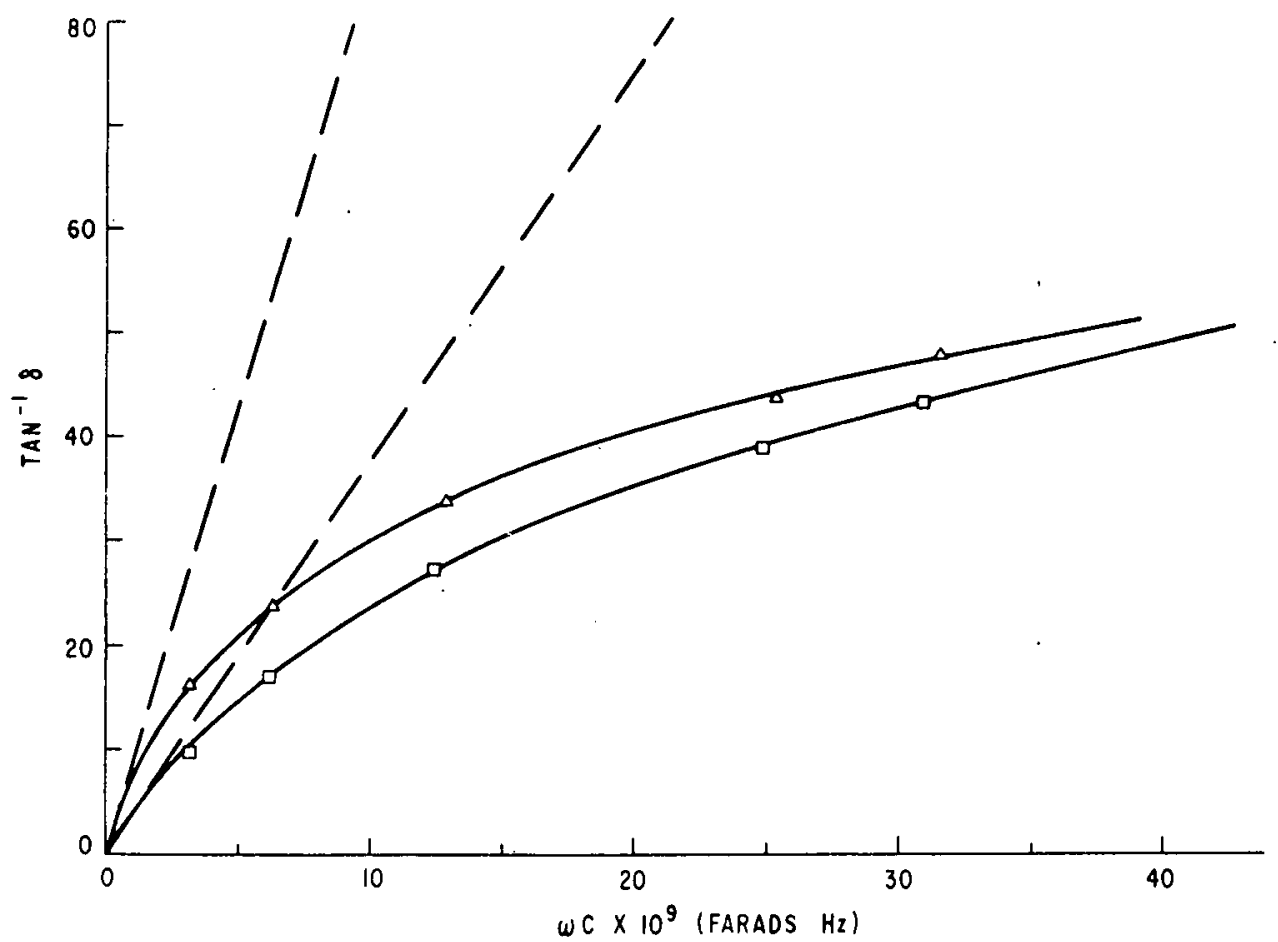

Fig. 7-45 Frequency dependence of the reciprocal of loss tangent. $\quad \mathrm{PE}+5 \%$ sodium lauryl sulfate. 


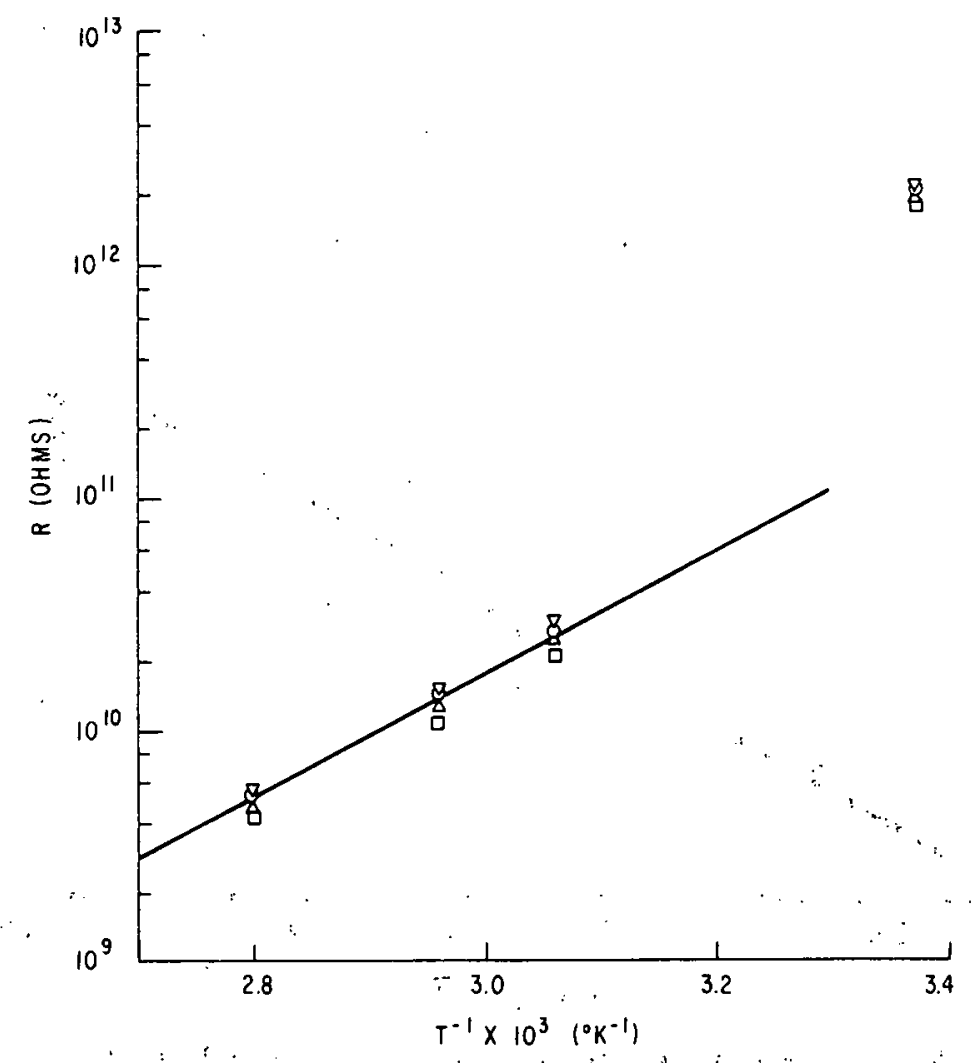

Fig. 7-46 Arrhenius plot. PE + 5\% sodium lauryl sulfate.

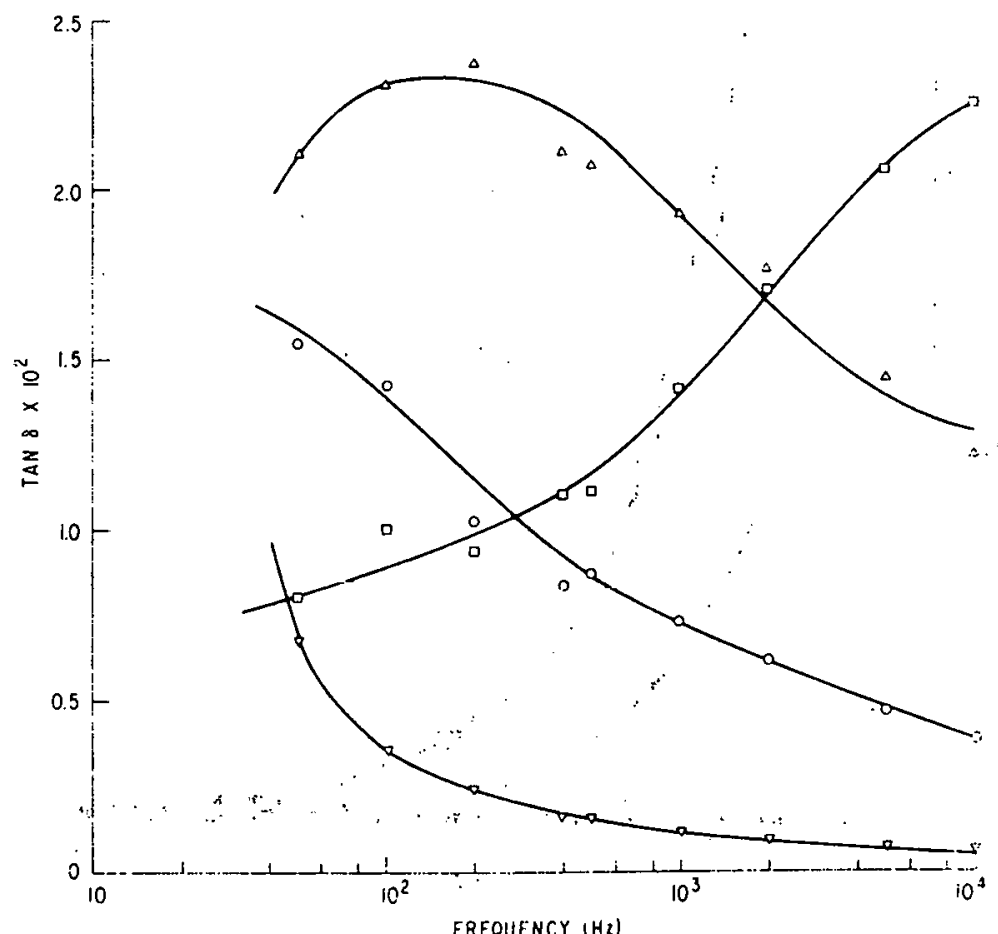

Fig. $7-47$ Frequency dependence of loss tangent. $\mathrm{PE}+{ }^{5}$ \% cetyltrimethylammonium bromide. 


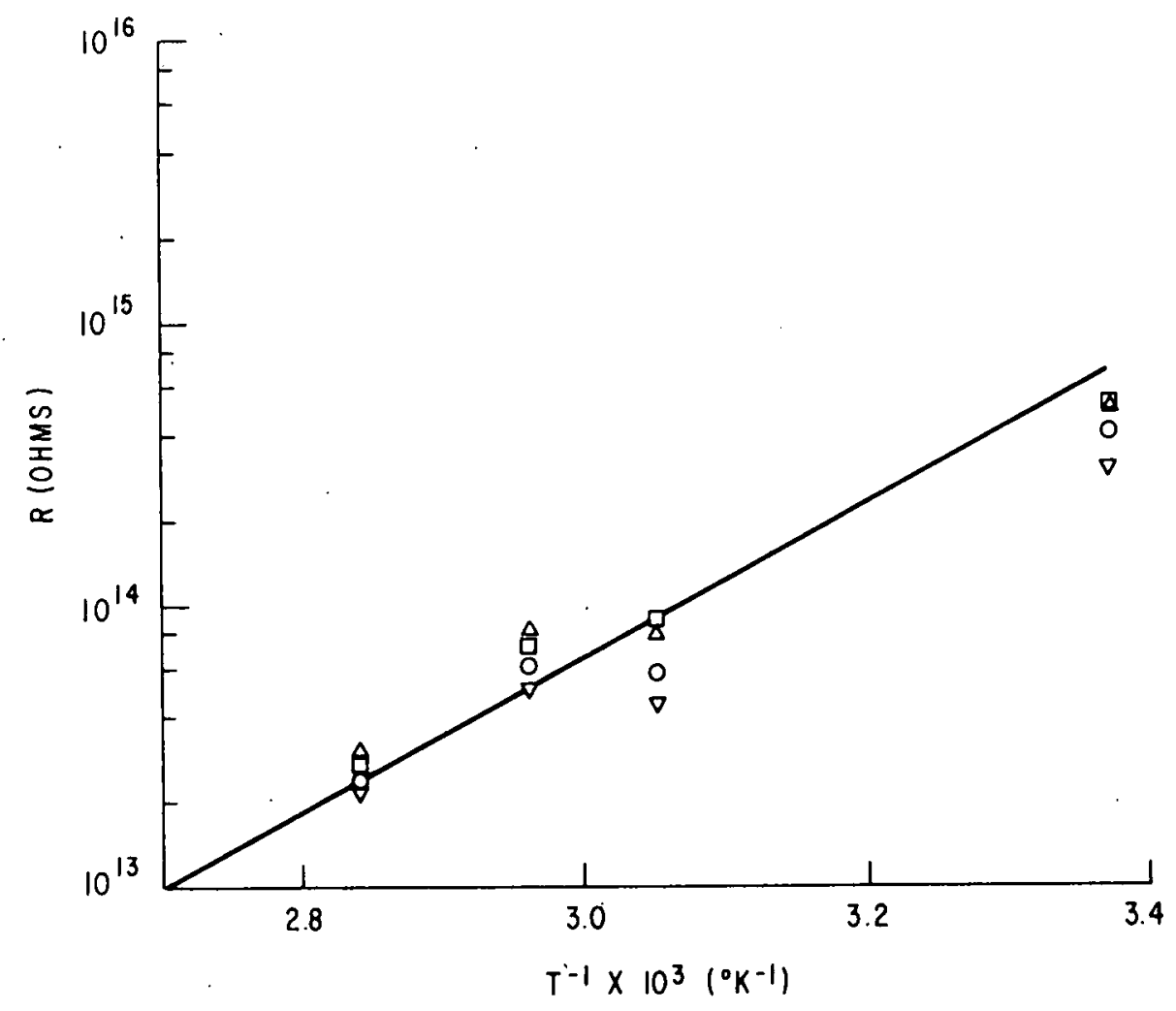

Fig. 7-48 Arrhenius plot. PE + 5\% cetyltrimethylammonium bromide.

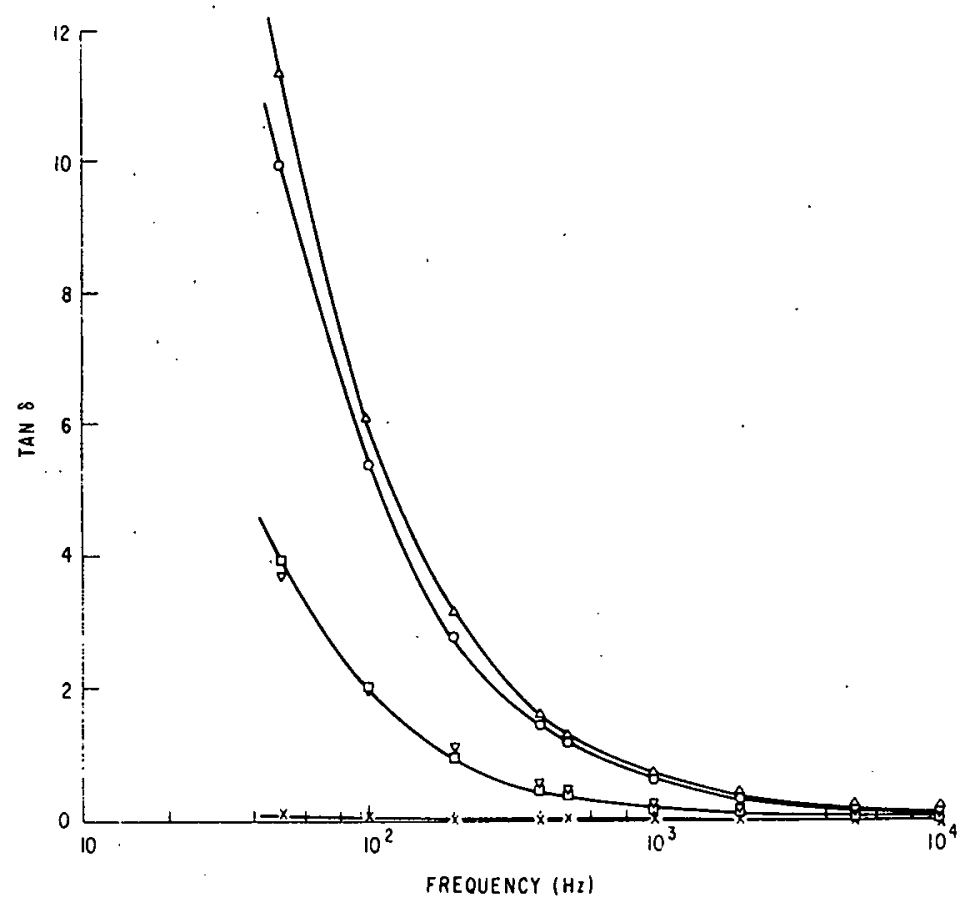

Fig. 7-49 Frequency dependence of loss tangent. PVAP + 5\% tetraheptylammonium chloride. $(\mathrm{x}): 25^{\circ} \mathrm{C}$ after temperature cycling. 


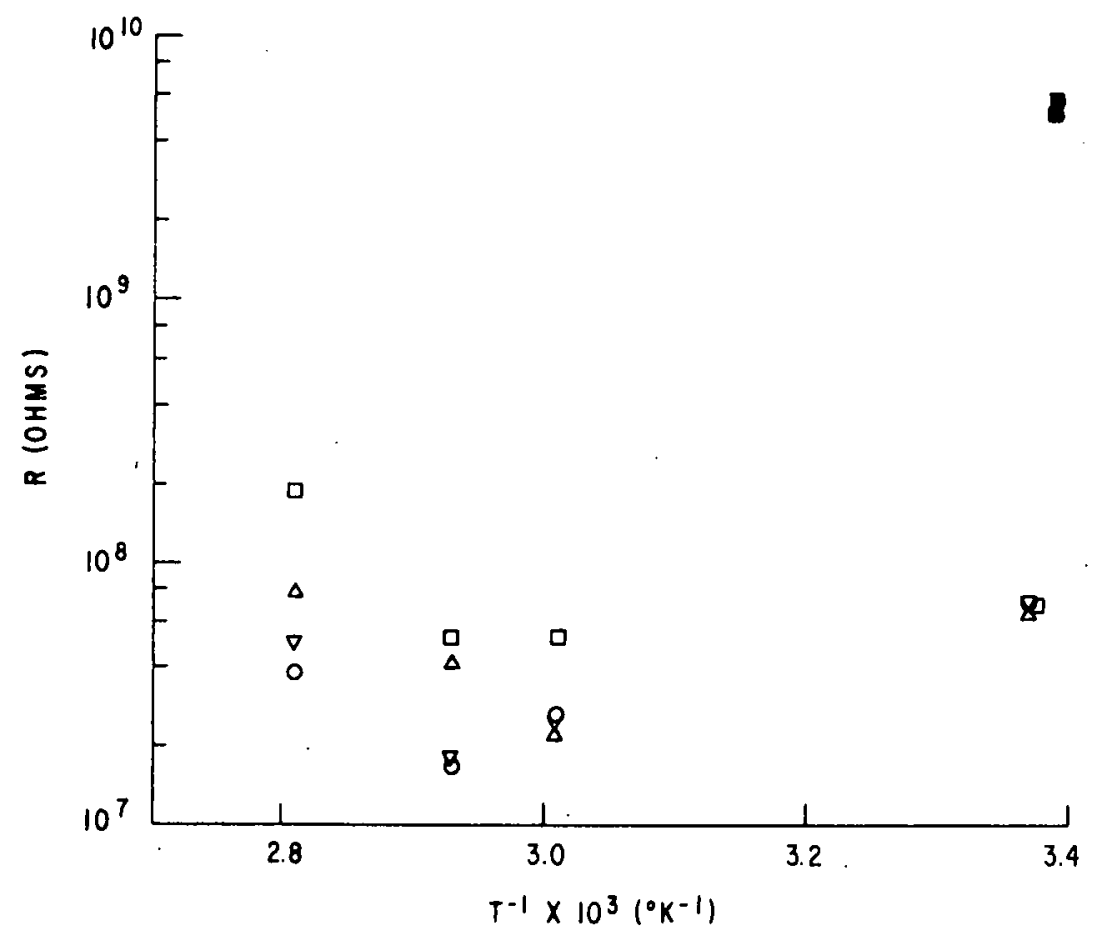

Fig. 7-50 Arrhenius plot. PVAP + 58 tetraheptylammonium chloride. Solid points $-25^{\circ} \mathrm{C}$ after temperature cycling.

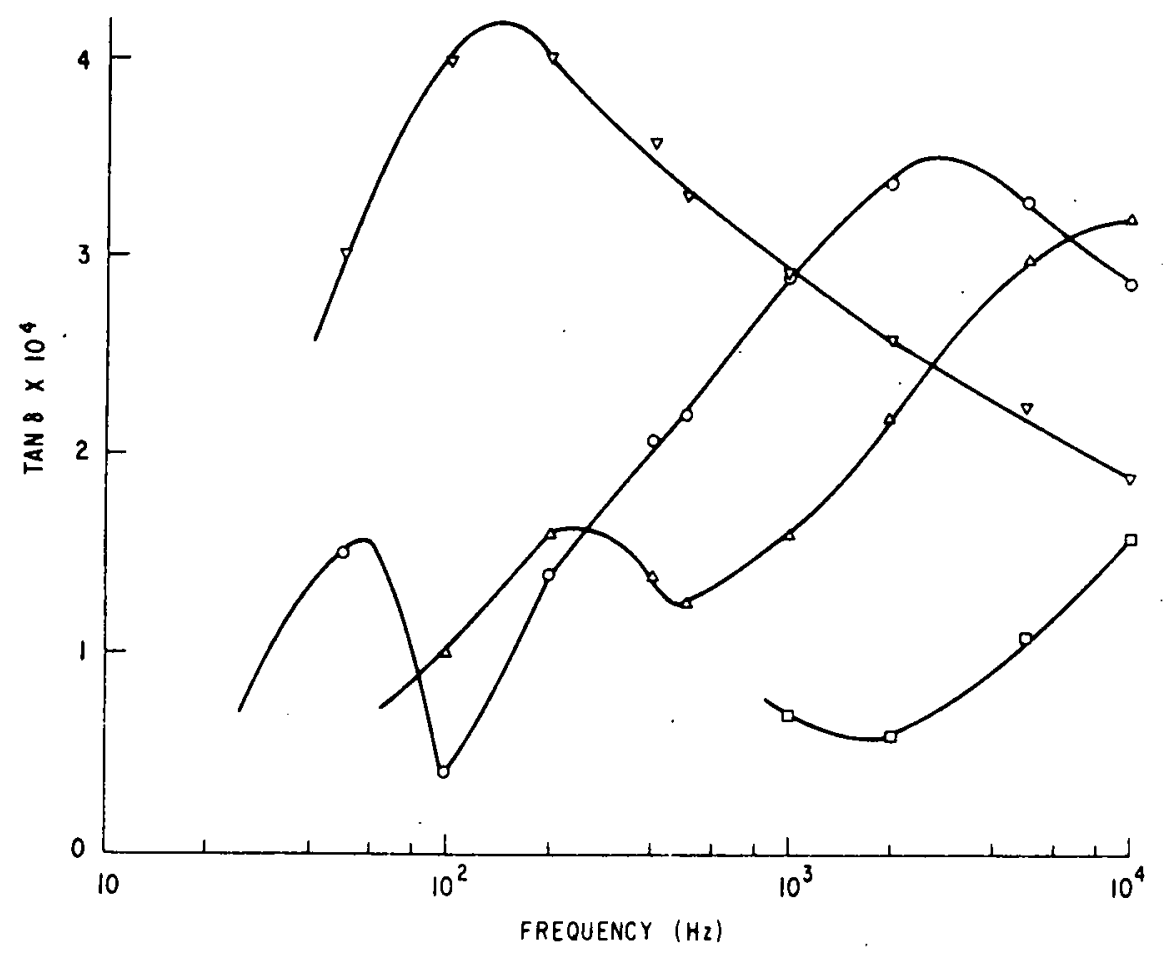

Fig. 7-51 Frequency dependence of loss tangent. $\mathrm{PE}+5 \%$ ferrocene. 